\title{
PHYSICAL AND CHEMICAL INVESTIGATIONS OF RICE AS RAW MATERIAL OF SAKÉ.
}

\author{
By Hisaye Satow.
}

ConTents.

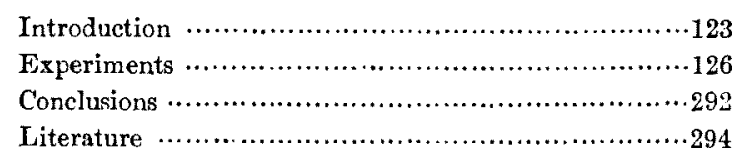

\section{INTRODUCTION.}

While the greater part of our output of rice is consumed as food-stuff, just a small part such as about six to seven per cent of it is estimated to be used as the raw material of Saké. Many physical and chemical researches of rice have hitherto been made from the stand point of food, but very few have been made as the raw material of Sake. From the former point of view O. Kellner, ${ }^{(1,2)}$ Y. Kozai, ${ }^{(33)}$ M. Nagaoka, ${ }^{(2,4)}$ M. Sawamura, ${ }^{(3,2,2,25)}$ S. Kato, ${ }^{(24)}$ I. Inagaki, $\left.{ }^{(9,}, 11,10\right)$ H. Ando, ${ }^{(33)}$ U. Suzuki, ${ }^{(7,8,9,13,13,33,39)}$ K. Aso, ${ }^{(9 .}$ 29, 32) and M. Kondo, $\left.{ }^{17,}, 18,20\right)$ and many others have made various useful researches, of which, the discovery of oryzanin made by U. Suzuki deserves special attention. From the latter view point i. e. as raw material of Saké, however, few reports exist such as made by $\mathrm{T}$. Takahashi, ${ }^{(43,44)} \mathrm{T}$. Tadokoro,

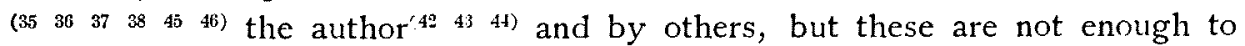
define the quantities of rice as the raw material of Saké.

The researches on rice as the food-stuff have been done in general as to the varieties, manures, soils, climates, and the mode of cultivation. The main results of physical and chemical researches in these respects are to be summarised as follows. Among the typical varieties belonging to large kernel may be mentioned Omachi, Miyako, Watashibune and Shiratama: among middle sized ones Shinriki, Aikoku, Ohmi, Ishiziro and Araki and among small varieties Shekitori and Shinshukaneko. The number of varieties amounts to almost ten thousands. Preference of the varieties of rice differs according to districts, viz. prefectures of Kwanto prefer small grains, on the other hand, prefectures of Kwansai prefer large one. From the standpoint of cultivation, varieties of small kernel are fitted to Tohoku prefectures and those of middle sized grain to Tokai, Tosan prefectures, while those of large grain are best for Chugoku, Shikoku and Kyushu prefectures. Regarding 
the nature of soil and climate, the best soil is sandy loam formed by the disintegration of granite, moreover, drainage must be good and abundant supply of irrigation water is necessary, while the quantity of rain during summer time must be small. Too much rain brings about the deficit of heat in consequence and the propagation of rice plant is injured. Clay soil is not entirely unfit for rice plantation, as loss of manure is small, and crop is abundant, but the quality of rice is expected to be inferior. Tohoku prefectures prefer varieties of the early crop, while prefectures of Shikoku and Kyushu rather prefer varieties of the later crop taking both the quality as well as quantity of the crop in consideration. When nitrogenous manure is given copiously, the yield of rice may be increased, but on the other hand, the quality of rice will generally be inferior, since the harvest is delayed, and the crop is mixed with a quantity of red rice and inferior one. When nitrogenous manure is lessend and phosphoric and potash manure is increased, the quality of rice may be superior, and the harvest earier, but the crop is decreased. If we aim to obtain both the best quality and the largest yield, we must give suitable quantities of nitrogenous, phosphatic and potash manures. Indirect manure, such as lime, when given in a greater quantity than normal, brittleness of rice grains may follow. Of chemical compositions, the quantities of starch and nitrogen must be great and those of water, cellulose and ash must be small. Since starch is the principal constituent of rice constituting the endosperm, there is no objection as to the assertion that the rice is better when rich in starch. Regarding nitrogen, the experiments hitherto made on the same varieties in the same climate, shows that the quality of rice is better when the quantity of nitrogen is the greater. Rice produced in India, Java and in several districts, where lime is abused contain less nitrogen and the quality is regarded to be inferior. Potassium, calcium, phosphorous, sodium, iron and magnesium are necessary ingredients as nutritious matter, but these are not taken into consideration in the judgment of the quality of rice. In regard to the fat, it is better that the rice contains it much, since the fat is indispensable for the human nutrition. Water contents ought to be small. Much water makes the percentage of other important constituents lower, diminishes the preservative power, produces, moreover, crushed grains during polishing. Cellulose is chiefly contained in the bran. Abundant content of it, is objectionable as it is unnecessary for the nutrition. After all, for the food purpose the quantities of starch, nitrogen and fat in rice are desirable to be large, while water, cellulose and ash contents ought to be as small as possible. Regarding the physical properties, the following conditions are necessary-purity, luster, light colour, high degree of hardness, thickness in bredth, thin bran layer, shallow longitudinal furrow, 
high specific gravity, heavy weight and less Harasiromai (the lateral pari of rice kernel is white and opaque).

- The selection of rice for the material of Sake brewing has been a matter of mere experience until now. They thought that rice must be ordinary rice produced on paddy field, not over ripened and harvested rather in an earier period. It must possess high degree of purity, luster, light colour, heavy weight, enough thickness, thin bran layer, shallow longitudinal furrow, moderate hardness, and humidity. Rice used in Nada district, belongs to the medium variety produced in prefectures of Settsu and Harima. If grains are too hard in consequence of extreme diying or less water contents or overripening, then abundant Dowaremai (rice grain cracked) will be produced. Such hard grains produce greater loss during polishing as broken grains. When such hard rice is used as Koji, the growth of mycelium of the Koji fungus is damaged and consequently the saccharification is insufficient during mashing, and the equilibrium among saccharification, fermentation, and acid formation is disturbed. In such a case, fermentation of mash begins comparatively earlier but it is weak and tedious so that the consumption of sugar is dull accompanied by a copious formation of acid and bad flavour, resulting in an inferior product. On the contrary, if grains are too soft in consequence of insufficient drying or unripeness, greater loss is produced also during polishing as broken grains. In such a case saccharification preceeds too quickly and the fermentation is retarded, resulting deep coloured, strongly acidic, sweet mash destitute of alcohol. In short, the rice to be used for Saké brewing must be of large, rather soft grains, and contain abundant starch. In other respects, no reliable data have yet been given.

Regarding the nature of soil, sandy loam, viz. granite soil is regarded to be best fitted for the rice plantation and those granite districts such as prefectures of Settsu, Harima and Bizen are thought to produce the best kind of rice.

As previously mentioned, the judgment of rice to be used for Saké brewing has been done chiefly concerning its physical properties. Probably the chemical changes of rice during Saké brewing are more or less complicated according to the treatment given by the brewer. So the quality of Sake is dependent not only on the quality of rice, but also on the skill of the brewer and other accidental circumstances. Suitable technical treatment is, of course, very important. Moreover, qualities of water and vat of the aging constitute important factors in the quality of the product, further more climate has an important influence on the fermentation. Apart from these causes, the quality of rice has a predominant relation to the quality of Sake produced. The choice of the rice for the brewing is not, however, a matter 
of concordance in different districts, as the brewers select their raw materials only from their experiences. It is beyond doubt that we wanted a reliable standard for the selection of rice as the raw material of Sake brewing. The author intended to investigate the numerous varieties of rice produced in several districts. The object of this investigation is to determine the physical and chemical properties of husked rice, which is regarded to be suitable for Saké brewing. The experiments have been conducted on 242 varieties including husked and polished rice, covering the production of five years.

\section{EXPERIMENTS.}

a) Percentage of the full grown grains:- $100 \mathrm{~g}$. of each sample were spread on a smooth black paper, all impurities except the full grown grains were collected and weighed. The weight was deducted from 100 . The remainder shows the percentage of the full grown grains.

b) External appearance and luster:-definite quantities of each sample were placed on a white paper side by side and their exterual appearance, luster and shade of colour were compared.

c) Size of grains. (mm.) :- 100 g. of each sample were first sifted through a sieve of perforations of $2.2 \mathrm{~mm}$. diameter and then of $2.0 \mathrm{~mm}$., $1.8 \mathrm{rrm}$. and finally of $1.6 \mathrm{~mm}$. Among them 50 grains were taken out in proportion, and their length and width were measured.

d) Percentage of Shinziromai (grains with opaque centre):- $-50 \mathrm{~g}$. of each sample were reflected through a diaphanoscope.

e) Depth of longitudinal furrow $(\mu):-10$ full grown grains were cut cross ways just in two parts with a razor, and the two furrows on both sides of the grains were measured under a microscope of a low power with an ocular micrometer.

f) Specific gravity:-20g. of each sample were plunged into a burette containing $90 \%$ alcohol, and the weight is divided by the c.c. of volume occupied.

g) Saccharifying quality:-One g. of powdered sample was added with 100c.c. of water and boiled for 30 minutes with a reverted cooler; when cooled, 52.5c.c. water and 7.5c.c. Koji extract were added. $25 \mathrm{~g}$. Koji, added with 50c.c. water were kept for 6 hours with stirring from time to time and then filtered.)

This mixture was kept in a thermostat of $28.5^{\circ} \mathrm{C}$. for 2 hours, then boiled, cooled, filled up to 200c.c. of which 10c.c. were used for the determination of sugar (A). As controle, 7.5c.c. of the above mentioned Koji extract, added with $152.5 \mathrm{c}$.c. water were kept in a thermostat of $28.5^{\circ} \mathrm{C}$. for 2 hours, treated as above, and the sugar contents were determined (B). 
Powdered rice was also analysed on sugar (C).

$$
\text { Sugar produced }=\mathrm{A}-(\mathrm{B}+\mathrm{C})
$$

h) Hardness (kilo) :-50 or 100 grains were tested with Ando's "Festigkeit Prüfer."

i) Volume of $100 \mathrm{~g}$. (c.c.):-_ Brauer's “Getreide Prüfer" was used.

j) Weight of 1,000 grains (g.):- 1,000 full grown grains were weighed.

k) Volume of 1,000 grains (c.c.) :-1,000 full grown grains were plunged into $90 \%$ alcohol and the occupied volume was determined.

l) Phytin :- measured according to J. B. Rather's method, (Phytin phosphorous as $\mathrm{P}_{2} \mathrm{O}_{5}$ in dry matter).

m) Thickness of bran layer $(\mu)$ (Pericarp, testa, perisperm and aleuron layer) :-_Each grain was cut vertically with a razor at 10 places, steeped in n/100 iod-iodpotassium solution, measured with an ocular micrometer. 20 grains of each sample were tested.

n) Absorption of water (\%):-_— To $50 \mathrm{~g}$. of rice were added 60c.c. of water and after the time mentioned, the sample was well squeezed from water between dry papers and weighed.

o) Depth of embryo cavity:-A grain was cut cross ways, with a razor into two equal parts, then longitudinally along the embryo cavity, steeped in $\mathrm{n} / 100$ iod-iodpotassium solution, and the depth of embryo cavity was measured with an ocular micrometer, using a microscope of a low power.

Thus the depth of the cavity is divided by the length of the cavity. 10 grains of each sample were used.

Samples used for experiments were always kept at 26 to $27^{\circ} \mathrm{C}$. methods.

p) Chemical analysis :_All constituents were measured by the usual Analyses of embryo.

$7.7 \mathrm{~g}$. of embryo were obtained from about $405 \mathrm{~g}$. of husked rice, the compositions of the embryo were as follows :-

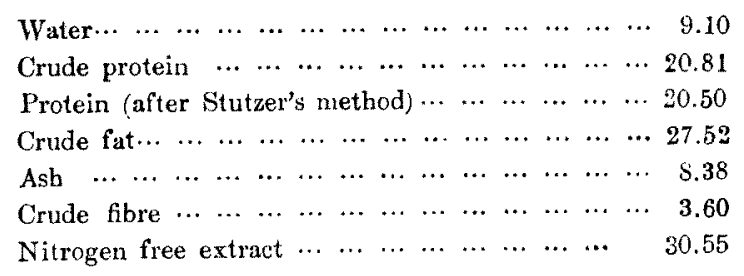

\section{Production.}

a) Husked rice, suitable for the Sake brewing. 18 (A-R) varieties.

A) Production of Okayama prefecture.

a) Percentage of full grown grains. - $91.0 \%$ 
b) External appearance and luster-grains were fat and large, having a straw yellow waxy luster, with practically no impurities.

c) S:ze of grains (m.m.)

\begin{tabular}{|c|c|c|c|c|}
\hline \multicolumn{2}{|c|}{ hickness above } & 2.2 & $70.36 \mathrm{~g}$. & $35 \mathrm{gra:n}$ \\
\hline " & " & 2,0 & $22.60 "$ & $11 " 1$ \\
\hline " & "I & 1.8 & $.5 .90 " \prime$ & $"$ \\
\hline " & under & $\begin{array}{c}1.8 \\
\text { average. }\end{array}$ & $\begin{array}{l}1.10 / 1 \\
\max .\end{array}$ & $\begin{array}{l}1 \prime \prime \\
\text { twin. }\end{array}$ \\
\hline & & 5.27 & 5.60 & 4.90 \\
\hline & & 2.98 & 3.20 & 2.80 \\
\hline
\end{tabular}

d) Percentage of Shingiromai. -55.64

e) Depth of longitudinal furrow. $(\mu)-$

$\begin{array}{ccc}\text { average. } & \max . & \min . \\ 52.76 & 87.50 & 35.0\end{array}$

f) Specific gravity.--1.404

.g) Saccharifying quality.-31.98\%

h) Hardness. (kilo)

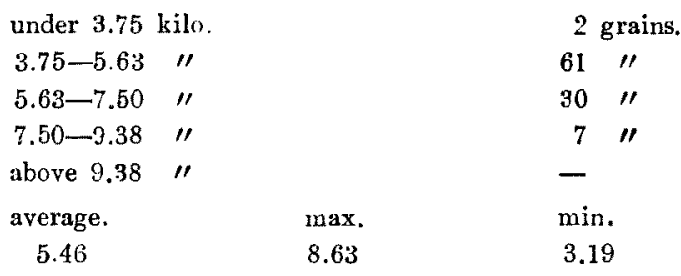

i) Volume of $100 \mathrm{~g}$. - $121.5 \mathrm{c} . \mathrm{c}$.

j) Weight of 1,000 grains.-26.22g.

$\mathrm{m})$ Thickness of bran layer. $(\mu)$

$\begin{array}{crr}\text { average } & \max . & \min . \\ 43.38 & 59.40 & 33.75\end{array}$

n) Absorption of water.

$\begin{array}{ccc}\text { time steeped. } & \text { water absorded. } & \% \text { absorbed. } \\ 1 \mathrm{~h} . & 2.20 & 4.40 \\ 3 & 4.90 & 9.80 \\ 24 & 11.47 & 22.94\end{array}$

c) Depth of embryo cavity :-
arerage.
$\max$
min.

$\begin{array}{lll}0.27 & 0.30 & 0.21\end{array}$

p) Chemical composition :-

Water

Direct R. sugar

Dextrin

Starch
13.22

2.37

2.01

72.17 unhydrows.

$$
\begin{array}{r}
2.61 \\
2.32 \\
83.17
\end{array}
$$




$\begin{array}{ccc}\text { Crude fat } & 1.76 & 2.03 \\ \prime \prime \text { fiber } & 1.23 & 1.42 \\ \text { " protein } & 6.70 & 7.72 \\ \text { Ash } & 1.16 & 1.34 \\ \text { Puosphoric acid } & 0.37 & 0.43\end{array}$

B) Production of Okayama prefecture.

a) Percentage of full grown grains.- - $94.1 \%$

b) Luster and external appearance--nearly same as A).

c) Size of grains. (m.m.)

\begin{tabular}{|c|c|c|c|c|c|}
\hline hick & above. & 2.2 & $82.10 \mathrm{~g}$. & 41 & rains \\
\hline$\prime \prime$ & 11 & 2.0 & $14.22 \prime \prime$ & 7 & $\prime \prime$ \\
\hline "I & $" \prime$ & 1.8 & $3.20 " \prime$ & 2 & $\prime \prime$ \\
\hline$" \prime$ & under & 1.8 & $0.40 " \prime$ & 一 & \\
\hline & & average & $\max$. & $\min$. & \\
\hline & ngth & 5.30 & 5.60 & 4.70 & \\
\hline & $\mathrm{dth}$ & 2.97 & 3.20 & 2.80 & \\
\hline
\end{tabular}

d) Percentage of Shinziromai._- $79.10 \%$

e) Depth of longitudinal furrow. $(\mu)$

$\begin{array}{crc}\text { average. } & \max . & \min . \\ 51.63 & 78.75 & 29.75\end{array}$

f) Specific gravity. $\quad 1.404$

g) Saccharifying quality. $\quad 35.41 \%$

h) Hardness. (kilo)

\begin{tabular}{|c|c|c|}
\hline under 3.75 & & 23 grains. \\
\hline $3.75-5.63$ & & $64 \quad \prime \prime$ \\
\hline $5.63-7.50$ & & 10 \\
\hline $7.50-9.38$ & & 3 \\
\hline - above 9.38 & & - \\
\hline $\begin{array}{c}\text { average. } \\
4.39\end{array}$ & $\begin{array}{l}\max . \\
8.86\end{array}$ & $\begin{array}{l}\min . \\
2.55\end{array}$ \\
\hline
\end{tabular}

i) Volume of $100 \mathrm{~g}$. $122.0 \mathrm{c} . \mathrm{c}$.

j) Weight of 1,000 grains. $-26.97 \mathrm{~g}$.

m) Thickness of bran layer. $(\mu)$

$\begin{array}{ccc}\text { average. } & \max . & \min . \\ 42.65 & 56.25 & 33.75\end{array}$

n) absorption of water.

$\begin{array}{ccc}\text { time steeped. } & \text { water adsorbed. } & \% \text { absorbed. } \\ 1 \mathrm{~h} . & 2.05 & 4.1 \\ 3 & 4.50 & 9.0 \\ 24 & 10.70 & 21.40\end{array}$

o) Depth of embryo cavity.

$\begin{array}{crc}\text { average. } & \max . & \min . \\ 0.25 & 0.29 & 0.19\end{array}$


p) Chemical composition

$\begin{array}{lrc} & & \text { unhydrous. } \\ \text { Water } & 13.99 & - \\ \text { Direct R. sugar } & 2.91 & 3.41 \\ \text { Dextrin } & 1.63 & 1.89 \\ \text { Starch } & 70.29 & 81.72 \\ \text { Crude fat } & 1.85 & 2.15 \\ \quad \text { " fibre } & 0.99 & 1.15 \\ \quad \text { " protein } & 6.88 & 8.00 \\ \text { Ash } & 1.16 & 1.35 \\ \text { Phosphoric acid } & 0.35 & 0.41\end{array}$

C) Production of Okayama prefecture.

a) Percentage of full grown grains.—-91.95\%

b) External appearance and luster-nearly same as A).

c) Size of grains. (m.m.)

\begin{tabular}{|c|c|c|c|c|c|}
\hline \multicolumn{2}{|c|}{ thickness above. } & 2.2 & $75.10 \mathrm{~g}$. & \multicolumn{2}{|c|}{38 grains. } \\
\hline$" \prime$ & $\prime \prime$ & 2.0 & $18.80 \mathrm{ll}$ & 9 & $\prime \prime$ \\
\hline$\prime \prime$ & $" \prime$ & 1.8 & $5.20 " \prime$ & 3 & $" \prime$ \\
\hline$\prime \prime$ & under. & 1.8 & $0.80^{\circ} " \prime$ & - & \\
\hline & ngth & $\begin{array}{c}\text { average. } \\
5.37\end{array}$ & $\begin{array}{l}\max . \\
5.70\end{array}$ & $\begin{array}{l}\min . \\
4.90\end{array}$ & \\
\hline & dth & 3.00 & 3.30 & 2.70 & \\
\hline
\end{tabular}

d) Percentage of Shinziromai :- $64.3 \%$

e) Depth of longitudinal furrow. $(\mu)$

$\begin{array}{ccr}\text { average. } & \max . & \min . \\ 46.74 & 87.50 & 17.50\end{array}$

f) Specific gravity.-1.389.

g) Saccharifying quality.-33.80\%

h) Hardness. (kilo)

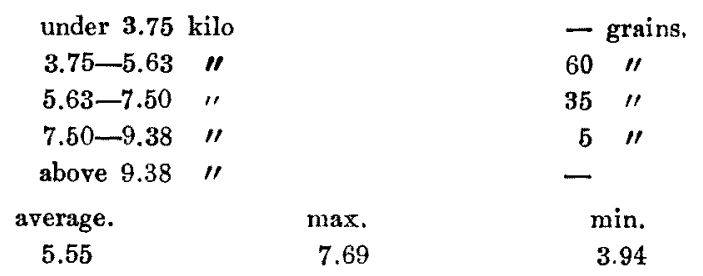

i) Volume of $100 \mathrm{~g} .-125.0 \mathrm{c} . \mathrm{c}$.

j) Weight of 1,000 grains. $-26.95 \mathrm{~g}$.

m) Thickness of bran layer. $(\mu)$

$\begin{array}{ccr}\text { average. } & \max . & \min . \\ 41.36 & 54.06 & 29.25\end{array}$

n) Absorption of water. 


$\begin{array}{ccc}\text { time steeped. } & \text { water absorbed. } & \% \text { absorbed. } \\ 1 \mathrm{~h} . & 2.05 \mathrm{gr} . & 4.1 \\ 3 & 4.75 \prime \prime & 9.50 \\ 24 & 10.39 \prime \prime & 20.60\end{array}$

o) Depth of embryo cavity.

$\begin{array}{ccc}\text { average. } & \max . & \min . \\ 0.26 & 0.34 & 0.23\end{array}$

p) Chemical composition :-

$\begin{array}{lrr}\text { Water } & 13.56 & - \\ \text { Direct R. sugar } & 1.55 & 1.80 \\ \text { Dextrin } & 1.38 & 1.59 \\ \text { Starch } & 72.65 & 84.05 \\ \text { Crude fat } & 2.13 & 2.47 \\ \quad \text { " fibre } & 1.01 & 1.17 \\ \quad \text { " protein } & 7.32 & 8.47 \\ \text { Ash } & 1.22 & 1.41 \\ \text { Phosphoric acid } & 0.37 & 0.43\end{array}$

D) Production of Osaka prefecture.

a) Percentage of full grown grains.- $92.3 \%$

b) External appearance and luster.—nearly same as A).

c) Size of grains. (m.m.)

\begin{tabular}{|c|c|c|c|c|c|}
\hline thick & above & 2.2 & $53.70 \mathrm{~g}$ & $27 \mathrm{~g}$ & grains \\
\hline$\prime \prime$ & $\prime \prime$ & 2.0 & 28.00 & 14 & $\prime \prime$ \\
\hline " & $" \prime$ & 1.8 & 17.10 & 9 & $" \prime$ \\
\hline " & under & 1.8 & 1.10 & - & \\
\hline & & average. & $\max$. & min. & \\
\hline & igth & 5.20 & 5.60 & 4.60 & \\
\hline & dth & 2,94 & 3.20 & 2.40 & \\
\hline
\end{tabular}

d) Percentage of Shinziromai._- $49.92 \%$

e) Depth of longitudinal furrow. (it)

$\begin{array}{clc}\text { average. } & \max . & \min . \\ 61.9 & 105.0 & 35.0\end{array}$

f) Specific gravity.-1.399

g) Saccharifying quality.—-29.93\%

h) Hardness. (kilo)

\begin{tabular}{|c|c|c|c|}
\hline under 3.75 & cilo & - & ains. \\
\hline $3.75-5.63$ & $\prime \prime$ & 33 & $\prime \prime$ \\
\hline $5.63-7.50$ & $\prime \prime$ & 49 & $\prime \prime$ \\
\hline $7.50-9.38$ & $\prime \prime$ & 18 & "I \\
\hline above 9.38 & & - & \\
\hline verage & $\max$ & & in. \\
\hline 6.28 & 9.26 & & .86 \\
\hline
\end{tabular}


i) Volume of $100 \mathrm{~g} \cdot-124.0 \mathrm{c} . \mathrm{c}$.

j) Weight of 1,000 grains. $-24.70 \mathrm{~g}$.

m) Thickness of bran layer. $(\mu)$

$\begin{array}{ccc}\text { average. } & \max . & \min . \\ 41.48 & 49.50 & 33.75\end{array}$

n) Absorption of water.

$\begin{array}{ccc}\text { time steeped. } & \text { water absorbed. } & \% \text { absorbed. } \\ 1 \mathrm{~h} . & 2.90 & 5.8 \\ 3 & 6.35 & 12.7 \\ 24 & 12.02 & 24.04\end{array}$

o) Depth of embryo cavity.

$\begin{array}{ccc}\text { average. } & \max . & \min . \\ 0.25 & 0.34 & 0.15\end{array}$

p) Chemical composition :-

$\begin{array}{lrc}\text { Water } & 12.64 & - \\ \text { Direct R. sugar } & \mathbf{2 . 4 2} & 2.77 \\ \text { Dextrin } & 1.92 & 2.20 \\ \text { Starch } & 71.17 & 81.47 \\ \text { Crude fat } & 2.19 & 2.51 \\ \quad \text { " fibre } & 1.02 & 1.17 \\ \quad \text { " protein } & 6.88 & 7.87 \\ \text { Ash } & 1.37 & 1.46 \\ \text { Phosphoric acid } & 0.60 & 0.69\end{array}$

E) Production of Hyogo prefecture.

a) Percentage of full grown grains.- $88.05 \%$.

b) External appearance and luster._nearly same as A), but rather of middle size.

c) Size of grains. (m.m.)

\begin{tabular}{|c|c|c|c|c|c|}
\hline thick & ss above & 2.2 & $23.50 \mathrm{~g}$. & 12 & rains. \\
\hline$" 1$ & " & 2.0 & " 44.40 & 22 & " \\
\hline " & " & 1.8 & 29.00 & 15 & " \\
\hline " & under & 1.8 & $2.90 "$ & 1 & " \\
\hline & length & $\begin{array}{c}\text { average. } \\
5.31\end{array}$ & $\begin{array}{l}\max . \\
5.70\end{array}$ & $\begin{array}{r}\min . \\
4.50\end{array}$ & \\
\hline & width & 2.97 & 3.20 & 2.50 & \\
\hline
\end{tabular}

d) Percentage of Shinziromai._- $79.7 \%$

e) Depth of longitudinal furrow. $(\mu)$

$\begin{array}{rrr}\text { average. } & \max . & \min . \\ 55.88 & 86.50 & 34.60\end{array}$

f) Specific gravity.- -1.399

g) Saccharifying quality.—32.83\%

h) Hardness. (kilo) 


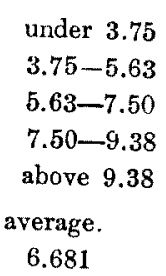

$$
\begin{aligned}
& 1 \text { grains. } \\
& 26 \text { " } \\
& 42 \text { " } \\
& 29 \text { " } \\
& 2 \quad \prime \\
& \text { min. } \\
& 3.4875
\end{aligned}
$$

i) Volume of $100 \mathrm{~g} . \longrightarrow 124.0 \mathrm{c} . \mathrm{c}$.

j) Weight of 1,000 grains.—24.20g.

$\mathrm{m})$ Thickness of bran layer. $(\mu)$

$\begin{array}{rrr}\text { average. } & \max . & \text { min. } \\ 43.97 & 59.40 & 36.00\end{array}$

n) Absorption of water.

$\begin{array}{ccc}\text { time steeped. } & \text { water absorbed. } & \% \text { absorbed. } \\ 1 \mathrm{~h} . & 2.68 & 5.36 \\ 3 & 5.67 & 11.34 \\ 21 & .12 .27 & 24.54\end{array}$

o) Depth of embryo cavity.

$\begin{array}{ccc}\text { average. } & \max . & \min . \\ 0.30 & 0.40 & 0.22\end{array}$

\begin{tabular}{|c|c|c|c|c|c|}
\hline \multicolumn{2}{|c|}{ thickness above } & 2.2 & $58.40 \mathrm{~g}$ & \multicolumn{2}{|c|}{29 grains } \\
\hline "I & "I & 2.0 & 33.00 , & 17 & $\prime \prime$ \\
\hline ", & $\prime \prime$ & 1.8 & 7.90 & 4 & $\prime \prime$ \\
\hline$\prime \prime$ & under & 1.8 & 0.60 & - & \\
\hline & length & $\begin{array}{c}\text { average. } \\
5.16\end{array}$ & $\begin{array}{l}\max . \\
5.60\end{array}$ & $\begin{array}{l}\min \\
4.60\end{array}$ & \\
\hline & width & 2.98 & 3.20 & 2.60 & \\
\hline
\end{tabular}

p) Chemical composition :-

$\begin{array}{lrr}\text { Water } & \mathbf{1 2 . 3 7} & - \\ \text { Direct R. sugar } & \mathbf{2 . 5 2} & 2.88 \\ \text { Dextrin } & 2.04 & \mathbf{2 . 3 3} \\ \text { Starch } & 70.39 & 80.33 \\ \text { Crude fat } & 2.25 & 2.57 \\ \quad \text { " fibre } & 1.03 & 1.18 \\ \quad \text { " prote'n } & 8.04 & 9.18 \\ \text { Ash } & \mathbf{1 . 4 2} & 1.62 \\ \text { Phosphoric acid } & 0.43 & 0.49\end{array}$

F) Production of Hyogo prefecture.

a) Percentage of full grown grains.- $96.7 \%$

b) External appearance and luster.—nearly same as A).

c) Size of grains. (m.m.)

d) Percentage of Shinziromai.- $83.0 \%$

e) Depth of longitudinal furrow. $(\mu)$ 


$$
\begin{gathered}
\text { under } 3.75 \\
\mathbf{3 . 7 5}-\mathbf{5 . 6 3} \\
5.63-7.50 \\
7.50-9.38 \\
\text { above } 9.38 \\
\text { average. } \\
5.64
\end{gathered}
$$$$
\max
$$$$
8.25
$$

i) Volume of $100 \mathrm{~g}$. - 119.0c.c.

j) Weight of 1,000 grains. $-25.10 \mathrm{~g}$.

m) Thickness of bran layer. $(\mu)$

$$
\begin{array}{rrr}
\text { average. } & \max . & \min . \\
45.72 & 51.75 & 39.15
\end{array}
$$

n) Absorption of water.

$\begin{array}{ccc}\text { time steeped. } & \text { water absorbed } & \% \text { absorbed } \\ \mathbf{1} \text { h. } & 1.80 & \mathbf{3 . 6 0} \\ \mathbf{3} & \mathbf{3 . 8 5} & 7.70 \\ \mathbf{2 4} & 10.30 & 20.60\end{array}$

o) Depth of embryo cavity.

\begin{tabular}{|c|c|c|c|c|c|}
\hline \multicolumn{2}{|c|}{ thickness above } & 2.2 & $49.80 \mathrm{~g}$. & \multicolumn{2}{|c|}{25 grains. } \\
\hline$\prime \prime$ & " & 2.0 & $35.20 " \prime$ & 18 & $" \prime$ \\
\hline$" \prime$ & $\prime \prime$ & 1.8 & $12.90 " \prime$ & 6 & " \\
\hline$" \prime$ & under & 1.8 & $1.90 "$ & 1 & $"$ \\
\hline & & average. & $\max$ & $\min$. & \\
\hline & ngth & 5.08 & 5.50 & 4.50 & \\
\hline & idth & 2.98 & 3.20 & 2.60 & \\
\hline
\end{tabular}

$\begin{array}{ccc}\text { average. } & \max . & \min . \\ 0.25 & 0.33 & 0.21\end{array}$

p) Chemical composition.-

$\begin{array}{lrr}\text { Water } & 12.88 & - \\ \text { Direct. R. sugar } & 3.16 & 3.63 \\ \text { Dextrin } & 1.26 & 1.42 \\ \text { Starch } & 70.59 & 81.03 \\ \text { Crude fat } & 2.37 & 2.72 \\ \quad \text { " fibre } & 0.99 & 1.14 \\ \quad \text { " protein } & 6.97 & 8.00 \\ \text { Ash } & 1.24 & 1.42 \\ \text { Phosphoric acid } & 0.37 & 0.41\end{array}$

G) Production of Kumamoto prefecture.

a) Percentage of full grown grains.- $83.8 \%$

b) External appearance and luster.—nearly same as A) but impurities were tolerably abundant.

c) Size of grains. (m.m.)

d) Percth of longitudinal furrow. $(\mu)$ 
$\begin{array}{rrr}\text { average. } & \max . & \min . \\ 60.98 & 86.50 & 43.25\end{array}$

f) Specific gravity. 1.384

g) Saccharifying quality._-30.29\%

h) Hardness. (kilo)

$$
\begin{gathered}
\text { under } 3.75 \\
3.75-5.63 \\
5.63-7.50 \\
7.50-9.38 \\
\text { above } 9.38
\end{gathered}
$$

average.

$\max$.

6.38

9.83

2 grains.

32 II

$44 \prime \prime$

$21 \quad \prime \prime$

$1 /$

min.

3.38

i) Volume of 100g. - 126.0c.c.

j) Weight of 1,000 grains. $-23.90 \mathrm{~g}$.

m) . Thickness of bran layer. $(\mu)$

$\begin{array}{rrr}\text { average. } & \max . & \min . \\ 45.74 & 67.50 & 36.45\end{array}$

n) Absorption of water.

$\begin{array}{ccc}\text { time steeped. } & \text { water absorbed } & \% \text { absorbed } \\ 1 \mathrm{~h} . & 2.45 & 4.9 \\ 3 & 4.97 & 9.94 \\ 24 & 10.57 & 21.14\end{array}$

o) Depth of embryo cavity.

$\begin{array}{ccc}\text { average. } & \max . & \min . \\ 0.26 & \mathbf{0 . 3 1} & 0.21\end{array}$

p) Chemical composition.-

$\begin{array}{lrr}\text { Water } & 13.15 & - \\ \text { D. R. sugar } & 2.06 & 2.38 \\ \text { Dextrin } & 2.23 & 2.57 \\ \text { Starch } & 71.80 & 82.67 \\ \text { Crude fat } & 2.08 & 2.40 \\ \quad \text { " fibre } & 1.13 & 1.30 \\ \quad \text { " protein } & 7.15 & 8.25 \\ \text { Ash } & 1.35 & 1.56 \\ \text { Phosphoric acid } & 0.40 & 0.45\end{array}$

\begin{tabular}{|c|c|c|c|c|c|}
\hline thicknes & above & 2.2 & $49.10 \mathrm{~g}$ & 25 & grains. \\
\hline$\prime \prime$ & 11 & 2.0 & 35.90 & 18 & $\prime \prime$ \\
\hline$\prime \prime$ & $\prime \prime$ & 1.8 & 12.40 & 6 & "I \\
\hline$\prime \prime$ & under & 1.8 & $2.20 \prime$ & 1 & $\prime \prime$ \\
\hline
\end{tabular}

H) Production of Yehime profecture.

a) Percentage of full grown grains.- $87.2 \%$.

b) External appearance and luster._-nearly same as A).

c) Size of grains. (m.m.) 


$\begin{array}{lccc} & \text { average. } & \text { max. } & \min . \\ \text { length } & 5.11 & 5.70 & 4.60 \\ \text { width } & 2.95 & 3.30 & 2.50\end{array}$

d) Percentage of Shinziromai.—-27.7 \%

e) Depth of longitudinal furrow. $(\mu)$

$\begin{array}{ccc}\text { average. } & \max . & \min . \\ 58.39 & 86.50 & 25.95\end{array}$

f) Specific gravity. - 1.394

g) Saccharifying quality. $-24.93 \%$

h) Hardness. (kilo)

\begin{tabular}{|c|c|c|c|}
\hline \multirow{2}{*}{\multicolumn{2}{|c|}{$\begin{array}{l}\text { under } 3.75 \text { kilo } \\
3.75-5.63\end{array}$}} & \multicolumn{2}{|c|}{5 grains. } \\
\hline & & 63 & $\prime \prime$ \\
\hline $5.63-7.50$ & & 30 & $\prime \prime$ \\
\hline $7.50-9.38$ & & 2 & " \\
\hline above 9.38 & & - & \\
\hline $\begin{array}{l}\text { average. } \\
5.23\end{array}$ & $\begin{array}{l}\max . \\
8.74\end{array}$ & & \\
\hline
\end{tabular}

i) Volume of $100 \mathrm{~g}$.-- $129.0 \mathrm{c} . \mathrm{c}$.

j) Weight of 1,000 grains. $-24.45 \mathrm{~g}$.

m) Thickness of bran layer. $(\mu)$

$\begin{array}{rrr}\text { average. } & \max . & \min . \\ 46.02 & \mathbf{5 6 . 2 5} & \mathbf{3 7 . 3 5}\end{array}$

n) Absorption of water.

$\begin{array}{ccc}\text { time steeped. } & \text { water absorbed. } & \% \text { absorbed. } \\ \mathbf{1} \text { h. } & 3.20 & 6.4 \\ 3 & 680 & 13.6 \\ 24 & 10.97 & \mathbf{2 1 . 9 4}\end{array}$

o) Depth of embryo cavity.

$\begin{array}{ccc}\text { average. } & \max . & \min . \\ 0.26 & 0.31 & 0.19\end{array}$

p) Chemical composition.-

$\begin{array}{lrr}\text { Water } & 13.86 & - \\ \text { D. R. sugar } & 2.42 & 2.80 \\ \text { Dextrin } & 1.33 & 1.55 \\ \text { Starch } & 71.52 & 83.03 \\ \text { Crude fat } & 2.07 & 2.40 \\ \quad \text { " fibre } & 1.20 & 1.39 \\ \quad \text { " protein } & 6.43 & 7.47 \\ \text { Ash } & 1.33 & 1.54 \\ \text { Phosphoric acid } & 0.54 & .0 .63\end{array}$

I) Production of Miye prefecture.

a) Percentage of full grown grains.-92.6\%

b) External appearance and luster.—nearly same as A). 
c) Size of grains. (m.m.)

thickness above

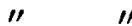

11

" under

$$
\text { length }
$$

width
2.2

2.0

1.8

1.8

average.

5.28

2.98

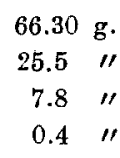

$\max$.

5.70

3.20
33 grains.

13 "

4 "

$\min$.

4.90

2.70

d) Percentage of Shinziromai._- $79.12 \%$

e) Depth of longitudinal furrow. $(\mu)$

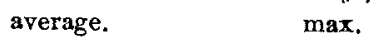

68.16

117.25

min.

35.0

f) Specific gravity._-1.399\% .

g) Saccharifying quality. $-37.99 \%$

h) Hardness. (kilo)

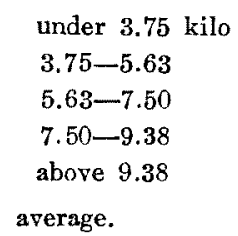

5.17

$\max$.

7.99

$$
\begin{array}{rr}
2 & \text { grains. } \\
70 & \prime \prime \\
24 & \prime \prime \\
4 & \prime \prime \\
- & \\
& \text { min. } \\
& 3.47
\end{array}
$$

i) Volume of $100 \mathrm{~g} . \longrightarrow 123.5$ c.c.

j) Weight of 1,000 grains. $-26.21 \mathrm{~g}$.

$\mathrm{m})$ Thickness of bran layer. $(\mu)$

$\begin{array}{ccc}\text { average. } & \max . & \min . \\ 46.31 & 67.50 & \mathbf{3 3 . 7 5}\end{array}$

n) Absorption of water.

$\begin{array}{ccc}\text { time steeped. } & \text { water absorbed. } & \% \text { absorbed. } \\ 1 \text { h. } & \mathbf{2 . 1 7} & 4.34 \\ \mathbf{3} & 4.90 & 9.30 \\ 24 & 10.77 & 21.54\end{array}$

o) Depth of embryo cavity.-

$\begin{array}{crr}\text { average. } & \max . & \min . \\ 0.28 & 0.35 & 0.33\end{array}$

p) Chemical composition.-

$\begin{array}{lrr}\text { Water } & 13.59 & - \\ \text { D. R. sugar. } & 2.26 & 2.62 \\ \text { Dextrin } & 2.04 & 2.36 \\ \text { Starch } & 71.53 & 82.79 \\ \text { Crude fat } & 1.96 & 2.27 \\ \quad \text { " fibre } & 1.02 & 1.18 \\ \quad \text { " protein } & 6.79 & 7.86\end{array}$




$\begin{array}{lll}\text { Ash } & \mathbf{1 . 1 2} & \mathbf{1 . 2 9} \\ \text { Phosphoric acid } & 0.36 & 0.41\end{array}$

J) Production of Niigata prefecture.

a) Percentage of full grown grains.-92.4\%

b) External appearance and luster.—somewhat greenish yellow, grains were not fat of midddle size, impurities were rare.

c) Size of grains. (m.m.).

\begin{tabular}{|c|c|c|c|c|c|}
\hline \multicolumn{2}{|c|}{ thickness above } & 2.2 & $44.10 \mathrm{~g}$. & 22 & rain: \\
\hline$" \prime$ & $\prime \prime$ & 2.0 & $36.5 \prime \prime$ & 18 & $\prime \prime$ \\
\hline$\prime \prime$ & $\prime \prime$ & 1.8 & 17.6 & 9 & $\prime \prime$ \\
\hline$\prime \prime$ & under & 1.8 & 1.5 & 1 & $\prime \prime$ \\
\hline & & average. & $\max$. & min. & \\
\hline & agth & 5.11 & 5.40 & 4.60 & \\
\hline & dth & 2.87 & 3.20 & 2.50 & \\
\hline
\end{tabular}

d) Percentage of Shinziromai.- $-8.84 \%$

e) Depth of longitudinal furrow. $(\mu)$
average.
$\max$.
min.
58.13
87.50
29.75

f) Specific gravity.-1.389.

g) Saccharifying quality. $-38.11 \%$

h) Hardness. (kilo)-

\begin{tabular}{|c|c|c|c|}
\hline under 3.75 & & - & \\
\hline $3.75-5.63$ & & 39 & grains. \\
\hline $5.63-7.50$ & & 58 & $\prime \prime$ \\
\hline $7.50-9.38$ & & 3 & $\prime \prime$ \\
\hline above 9.38 & & - & \\
\hline $\begin{array}{c}\text { average. } \\
5.88\end{array}$ & $\begin{array}{r}\max . \\
7.73\end{array}$ & & $\begin{array}{l}\min . \\
3.94\end{array}$ \\
\hline
\end{tabular}

i) Volume of $100 \mathrm{~g} . \longrightarrow 128.5 \mathrm{c} . \mathrm{c}$.

j) Weight of 1,000 grains.-23.44g.

m) Thickness of bran layer. $(\mu)$

$\begin{array}{ccr}\text { average. } & \max . & \min . \\ 54.61 & 96.75 & 36.00\end{array}$

n) Absorption of water.

$\begin{array}{ccc}\text { time steeped. } & \text { water absorbed. } & \% \text { absorbed } \\ 1 \mathrm{~h} . & 3.68 & 7.36 \\ 3 & 7.25 & 14.50 \\ 24 & 11.00 & 27.00\end{array}$

o) Depth of embryo cavity.

$\begin{array}{ccc}\text { average. } & \max . & \min . \\ 0.25 & 0.33 & 0.20\end{array}$

p) Chemical composition.- 


$\begin{array}{lrc} & & \text { unhydrous. } \\ \text { Water } & 13.97 & - \\ \text { D. R. sugar } & 2.24 & 2.60 \\ \text { Dextrin } & 1.83 & 2.13 \\ \text { Starch } & 69.80 & 81.13 \\ \text { Crude fat } & 2.08 & 2.41 \\ \quad \text { " fibre } & 0.99 & 1.15 \\ \quad \text { " protein } & 7.51 & 8.73 \\ \text { Ash } & 1.29 & 1.49 \\ \text { Phosphoric acid } & 0.40 & 0.47\end{array}$

K) Production of Kagawa prefecture.

a) Percentage of full grown grains._- $85.26 \%$

b) External appearance and luster.-_-nearly same as J).

c) Size of grains. (m.m.)

\begin{tabular}{|c|c|c|c|c|c|}
\hline \multicolumn{2}{|c|}{ thickness above } & 2.2 & $62.20 \mathrm{~g}$. & \multicolumn{2}{|c|}{31 grains } \\
\hline$\prime \prime$ & " & 2.0 & $27.90 " \prime$ & 14 & $\prime \prime$ \\
\hline " & $\prime \prime$ & 1.8 & $8.90 " \prime$ & 4 & " \\
\hline$\prime \prime$ & under & 1.8 & $1.00 " \prime$ & 1 & " \\
\hline & agth & $\begin{array}{c}\text { average. } \\
5.20\end{array}$ & $\begin{array}{l}\max . \\
5.60\end{array}$ & $\begin{array}{r}\min . \\
4.40\end{array}$ & \\
\hline & dth & 2.95 & 3.10 & 2.60 & \\
\hline
\end{tabular}

d) Percentage of Shinziromai.- $63.04 \%$

e) Depth of longitudinal furrow. $(\mu)$

$\begin{array}{ccr}\text { average. } & \max . & \min . \\ 50.69 & 95.15 & 25.95\end{array}$

f) Specific gravity.- 1.4056

g) Saccharifying quality.-36.16\%

h) Hardness. (kilo)

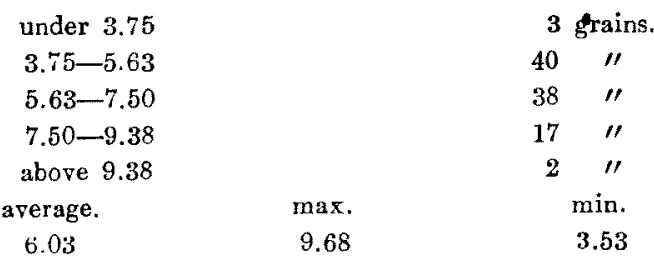

i) Volume of $100 \mathrm{~g} .-124.5$ c.c.

j) Weight of 1,000 grains.—25.41g.

$\mathrm{m})$ Thickness of bran layer. $(\mu)$

$$
\begin{array}{ccc}
\text { average. } & \max . & \min . \\
45.63 & 56.25 & 37.58
\end{array}
$$

n) Absorption of water.

$\begin{array}{ccc}\text { time steeped. } & \text { water absorbed. } & \% \text { absorbed. } \\ 1 \mathrm{~h} . & 2.40 & 4.8 \\ 3 & 5.60 & 11.2 \\ 24 & 11.85 & 23.70\end{array}$


o) Depth of embryo cavity.

$\begin{array}{ccc}\text { average. } & \max & \min . \\ 0.24 & 0.26 & 0.19\end{array}$

h) Chemical composition.-

$\begin{array}{lrc}\text { Water } & 13.04 & - \\ \text { D. R. sugar } & 1.19 & 1.37 \\ \text { Dextrin } & 1.12 & 1.42 \\ \text { Starch } & 72.07 & 82.86 \\ \text { Crude fat } & 2.11 & 2.43 \\ \quad \text { " fibre } & 1.17 & 1.35 \\ \quad \text { " protein } & 7.44 & 8.49 \\ \text { Ash } & 1.22 & 1.40 \\ \text { Phosphoric acid } & 0.34 & 0.39\end{array}$

L) Production of Miyagi prefecture.

a) Percentage of full grown grains.-69.3\%

b) External appearance and luster.-Somewhat deep straw brown yellow, no luster, grains were large and fat, impurities were abundant.

c) Size of grain. (m.m.)

\begin{tabular}{|c|c|c|c|c|c|}
\hline thickness & above & 2.2 & $76.50 \mathrm{~g}$. & \multicolumn{2}{|c|}{38 grains. } \\
\hline$\prime \prime$ & $" \prime$ & 2.0 & $16.10 "$ & 8 & $" \prime$ \\
\hline " & under & $\begin{array}{c}2.0 \\
\text { average. }\end{array}$ & $\begin{array}{l}7.40 " ~ \\
\text { max. }\end{array}$ & $\begin{array}{c}4 \\
\min \end{array}$ & $"$ \\
\hline & gth & 5.00 & 5.4 & 4.5 & \\
\hline & th & 3.10 & 33 & 28 & \\
\hline
\end{tabular}

d) Percentage of Shinziromai.-3.2\%

e) Depth of longitudinal furrow. $(\mu)$
average.
$\max$.
$\min$.
61.95
122.5
26.25

f) Specific gravity.-1.389.

g) Saccharifying quality. $-32.68 \%$

h) Hardness. (kilo)

$\begin{array}{lrrr}\text { under. } 3.75 \text { kilo. } & & - & \\ 3.75-5.63 & & 19 & \text { grains. } \\ 5.63-7.50 & & 29 & \prime \prime \\ 7.50-9.38 & & 2 & \prime \prime \\ \text { above } 9.38 & & - & \\ \text { average. } & \max . & & \mathrm{min} . \\ 5.80 & 7.97 & & 3.94\end{array}$

i) Volume of $100 \mathrm{~g}$. - 129.0c.c.

j) Weight of 1,000 grains. $-26.10 \mathrm{~g}$.

m) Thickness of bran layer. $(\mu)$

$\begin{array}{ccc}\text { average. } & \max . & \min . \\ 52.18 & 85.50 & 33.75\end{array}$


n) Absorption of water.

$\begin{array}{ccc}\text { time steeped. } & \text { water absorbed. } & \% \text { absorbed. } \\ 1 \text { h. } & 3.10 & 6.20 \\ 3 & 6.45 & 12.90 \\ 24 & 10.32 & 20.64\end{array}$

o) Depth of embryo cavity.

$\begin{array}{crr}\text { average. } & \max . & \min , \\ 0: 26 & 0.31 & 0.21\end{array}$

p) Chemical composition.

$\begin{array}{lrc} & & \text { unhydrous. } \\ \text { Water } & 16.09 & - \\ \text { D. R. sugar } & 2.67 & 3.18 \\ \text { Dextrin } & 2.18 & 2.60 \\ \text { Starch } & 6842 & 81.54 \\ \text { Crude fat } & 0.61 & 0.73 \\ \quad \prime \quad \text { fibre } & 0.98 & 1.17 \\ \quad \text { " protein } & 8.58 & 10.22 \\ \text { Asb } & 1.50 & 1.79 \\ \text { Phosphoric acid } & 0.43 & 0.51\end{array}$

M) Production of Fukushima prefecture.

a) Percentage of full grown grains.-93.06\%

b) External appearance and luster._-Straw yellow waxy luster, grains were fat and somewhat small, impurities were rare.

c) Size of grains. (m.m.)

\begin{tabular}{|c|c|c|c|c|c|}
\hline thick & s above & 2.2 & $8.3 \mathrm{~g}$. & & ains \\
\hline "I & $" 1$ & 2.0 & $45.0 "$ & 23 & $"$ \\
\hline " & " & 1.8 & $44.7 \prime \prime$ & 22 & $" \prime$ \\
\hline !" & under & $\begin{array}{c}1.8 \\
\text { average. }\end{array}$ & $\begin{array}{l}1.9 " \\
\text { max. }\end{array}$ & $\begin{array}{c}1 \\
\min .\end{array}$ & " \\
\hline & ength & 4.84 & 5.30 & 4.30 & \\
\hline & ridth & 2.71 & 3.00 & 2.40 & \\
\hline
\end{tabular}

d) Percentage of Shinziromai- $-3.6 \%$

e) Depth of longitudinal furrow. $(\mu)$

$\begin{array}{rcr}\text { average. } & \max . & \min . \\ 42.64 & 69.20 & 17.30\end{array}$

f) Specific gravity.-1.394

g) Saccharifying quality.-27.21\%

h) Hardness. (kilo)

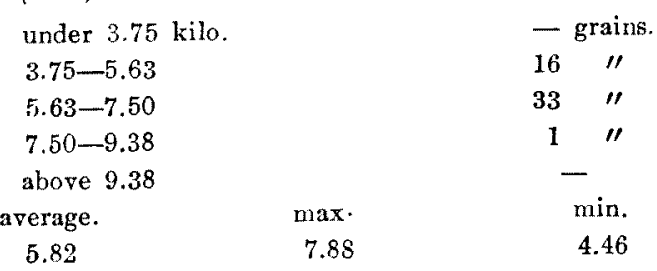


i) Volume of $100 \mathrm{~g}$.- $128.5 \mathrm{c} . \mathrm{c}$.

j) Weight of 1,000 grains.—20.2c.c.

in) Thickness of bran layer. $(\mu)$

$$
\begin{array}{ccc}
\text { average. } & \max . & \min . \\
42.49 & 63.0 & 31.5
\end{array}
$$

n) Absorption of water.

$\begin{array}{ccc}\text { time steeped. } & \text { water absorbed. } & \% \text { absorbed. } \\ 1 \text { 'h. } & 2.90 & 5.8 \\ 3 & 5.95 & 11.9 \\ 24 & 10.20 & 20.4\end{array}$

o) Depth of embryo cavity.

$\begin{array}{ccc}\text { average. } & \max . & \min . \\ 0.23 & 0.28 & 0.19\end{array}$

p) Chemical composition.

$\begin{array}{lrc} & & \text { unbydrous } \\ \text { Water } & 14.09 & - \\ \text { D. R. sugar } & 5.14 & 5.98 \\ \text { Dextrin } & 1.96 & 2.28 \\ \text { Starch } & 68.41 & 79.64 \\ \text { Crude fat } & 1.67 & 1.94 \\ \quad \text { " fibre } & 0.87 & 1.01 \\ \quad \text { " protein } & 7.77 & 9.04 \\ \text { Ash } & 1.31 & 1.52 \\ \text { Phosphoric acid } & 0.37 & 0.44\end{array}$

N) Production of Fukushima prefecture.

a) Percentage of full grown grains.- $75.4 \%$

b) External appearance and luster.-Straw yellow greyish colour, no luster, grains were small and fat, impurities tolerably abundant.

\begin{tabular}{|c|c|c|c|c|c|}
\hline thickne & is above & 2.2 & $25.4 \mathrm{~g}$. & 13 & rains \\
\hline " & " & 3.0 & $34.5 \prime \prime$ & 17 & " \\
\hline " & " & 1.8 & $28.5 "$ & 14 & $"$ \\
\hline$\prime \prime$ & under & 1.8 & $11.4 \prime \prime$ & 6 & " \\
\hline & ength & $\begin{array}{c}\text { average. } \\
4.86\end{array}$ & $\begin{array}{c}\max . \\
5.20\end{array}$ & $\begin{array}{r}\min . \\
4.30\end{array}$ & \\
\hline & idth & 2.86 & 300 & 2.50 & \\
\hline
\end{tabular}

c) Size of grains. (m.m.)

d) Percentage of Shinziromai.-4.4\%

e) Depth of longitudinal furrow. $(\mu)$

$\begin{array}{rrr}\text { average. } & \max . & \min . \\ 59.43 & 95.15 & 34.60\end{array}$

f) Specific gravity. -1.389 .

g) Saccharifying quality. -38.21

h) Hardness. (kilo) 


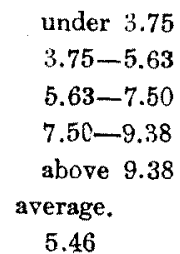

under 3.75

$3.75-5.63$

$5.63-7.50$

above 9.38

$\max$.

7.20

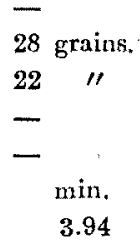

i) Volume of $100 \mathrm{~g} . \longrightarrow 133.5 \mathrm{c} . \mathrm{c}$.

j) Weight of 1,000 grains. $21.55 \mathrm{~g}$.

m) Thickness of bran layer. $(\mu)$

$\begin{array}{ccc}\text { average. } & \max . & \min . \\ 43.75 & 60.75 & 36.0\end{array}$

n) Absorption of water.

$\begin{array}{ccc}\text { time steeped. } & \text { water absorbed. } & \% \text { absorbed. } \\ 1 \text { h. } & 4.55 & 9.10 \\ 3 & 8.15 & 16.30 \\ 24 & 11.15 & 22.30\end{array}$

o) Depth of embryo cavity.

$\begin{array}{ccc}\text { average. } & \max . & \text { min. } \\ 0.26 & 0.30 & 0.23\end{array}$

p) Chemical composition.-

$\begin{array}{lrr}\text { Water } & 14.97 & - \\ \text { D. R. sugar } & 2.14 & 2.51 \\ \text { Dextrin } & 2.02 & 2.38 \\ \text { Starch } & 69.89 & 82.20 \\ \text { Crude fat } & 1.89 & 2.23 \\ \quad \text { " fibre } & 0.84 & 0.99 \\ \quad \text { " protein } & 7.06 & 8.30 \\ \text { Ash } & 1.25 & 1.47 \\ \text { Phosphoric acid } & 0.37 & 0.43\end{array}$

O) Production of Fukushima prefecture.

a) Percentage of full grown grains. $-89.6 \%$

b) External appearance and luster._- Straw brown yellow waxy luster, grains were fat and middle size, impurities were rare.

c) Size of grains. (m.m.)

$\begin{array}{ccc}\text { thickness above } & 2.2 \\ \prime \prime & \text { "I } & 2.0 \\ " \prime & \text { "l } & 1.8 \\ \prime \prime & \text { under } & 1.8 \\ & & \text { average. } \\ & \text { length. } & 4.90 \\ & \text { width } & 2.92\end{array}$

\begin{tabular}{|c|c|c|}
\hline $52.60 \mathrm{~g}$. & 278 & rains \\
\hline $34.10 " /$ & 17 & $\prime \prime$ \\
\hline $12.00 / l$ & 6 & $\prime \prime$ \\
\hline $1.10 " /$ & - & \\
\hline $\max$. & $\min$. & \\
\hline 5.40 & 4.30 & \\
\hline 3.20 & 2.70 & \\
\hline
\end{tabular}

d) Percentage of Shinziromai.-14.0\%

e) Depth of longitudinal furrow. $(\mu)$ 


$\begin{array}{ccc}\text { average. } & \max . & \min . \\ 54.24 & 74.39 & 34.60\end{array}$

f) Specific gravity.-1.389

g) Saccharifying quality._-20.15\%

h) Hardness. (kilo)

\begin{tabular}{|c|c|c|c|}
\hline under 3.75 & & \multicolumn{2}{|c|}{2 grains } \\
\hline $3.75-5.63$ & & 25 & $" \prime$ \\
\hline $5.63-7.50$ & & 22 & $\prime \prime$ \\
\hline $7.50-9.38$ & & 1 & $"$ \\
\hline above 9.38 & & - & \\
\hline $\begin{array}{c}\text { verage. } \\
5.42\end{array}$ & $\begin{array}{r}\max . \\
8.03\end{array}$ & & ain. \\
\hline
\end{tabular}

i) Volume of $100 \mathrm{~g}$. 129.5 c.c.

j) Weight of 1,000 grains. $-23.5 \mathrm{~g}$.

m) Thickness of bran layer. $(\mu)$

average.

46.05

n) Absorption of water.-

time steeped. water absorbed.

$1 \mathrm{~h}$.

3

24

3.22

6.50

10.70

o) Depth of embryo cavity. average.

0.29

$\max$.

0.37

p) Chemical composition.-

$\begin{array}{lr}\text { Water } & 14.36 \\ \text { D. R. sugar } & 3.40 \\ \text { Dextrin } & 2.25 \\ \text { Starch } & 71.01 \\ \text { Crude fat } & 1.77 \\ \quad \prime \text { fibre } & 1.20 \\ \prime \prime \text { protein } & 6.34 \\ \text { Asb } & 1.29 \\ \text { Phosphoric acid } & 0.46 \\ \text { of Fulustima prefecture. }\end{array}$

P) Production of Fukushima prefecture.
34.60 $\min$.

33.75

$\%$ absorbed.

6.44

13.00

21.40

min.

0.22

unhydrous.

$$
\begin{array}{r}
\overline{1} \\
3.97 \\
2.62 \\
82.92 \\
2.07 \\
1.40 \\
7.41 \\
1.51 \\
0.54
\end{array}
$$

a) Percentage of full grown grains.- $87.2 \%$

b) External appearance and luster.- Sonewhat dark straw yellow colour, no luster, grains ware fat and of middle size, impurities were tolerably abundant.

c) Size of grains. (m.m.)

$\begin{array}{cccccc}\text { thickness } & \text { above } & 2.2 & 76.60 \mathrm{~g} . & 38 \text { grains. } \\ \text { " } & \prime \prime & 2.0 & 18.50 " & 9 & \text { " } \\ \text { " } & \text { under } & 2.0 & 4.80 " & 3\end{array}$




$\begin{array}{lccc} & \text { average. } & \max . & \min . \\ \text { length } & 4.87 & 5.20 & 4.40 \\ \text { width } & \mathbf{3 . 0 2} & \mathbf{3 . 1 0} & 2.70\end{array}$

d) Percentage of Shinziromai.-4.2\%

e) Depth of longitudinal furrow. $(\omega)$ average. $\max$.

$\begin{array}{lll}47.49 & 86.5 & 25.95\end{array}$

f) Specific gravity. 1.389

g) Saccharifying quality.- $23.37 \%$

h) Hardness. (kilo)

\begin{tabular}{|c|c|c|c|}
\hline under 3.75 & & 2 & grain \\
\hline $3.75-5.63$ & & 30 & $\prime \prime$ \\
\hline $5.63-7.50$ & & 18 & " \\
\hline $7.50-9.38$ & & - & \\
\hline above 9.38 & & - & \\
\hline average. & $\max$. & & $\min$. \\
\hline
\end{tabular}

i) Volume of $100 \mathrm{gr}$. $130.0 \mathrm{c} . \mathrm{c}$.

j) Weight of 1,000 grains. $-24.7 \mathrm{~g}$.

m) Thickness of bran layer. $(\mu)$

$\begin{array}{ccc}\text { average. } & \max . & \min . \\ 48.56 & 65.25 & 40.50\end{array}$

n) Absorption of water.

$\begin{array}{ccc}\text { time steeped, } & \text { water absorbed. } & \text { \% absorbed. } \\ 1 \text { h. } & 2.65 & 5.30 \\ 3 & 5.55 & 11.10 \\ 24 & 10.15 & 20.30\end{array}$

o) Depth of embryo cavity.

$\begin{array}{crr}\text { average. } & \max . & \min . \\ 0.29 & 0.34 & 0.24\end{array}$

p) Chemical composition.-

$\begin{array}{lrr}\text { Water } & 15.57 & - \\ \text { D. R. sugar } & 2.18 & 2.59 \\ \text { Dextrin } & \mathbf{2 . 1 9} & 2.59 \\ \text { Starch } & 68.63 & 81.35 \\ \text { Crude fat } & 0.68 & 0.81 \\ \quad \text { " fibre } & 1.13 & 1.33 \\ \quad \text { " protein } & 8.13 & 9.63 \\ \text { Ash } & 1.36 & 1.61 \\ \text { Phosphoric acid } & 0.43 & 0.51\end{array}$

Q) Production of Yamagata prefecture.

a) Percentage of full grown grains. $\quad 89.64 \%$

b) External appearance and luster.-Grey yellow straw colour, no 
luster, grains were small and fat, impurities were tolerably abundant.

c) Size of grains. (m.m.)

\begin{tabular}{|c|c|c|c|c|c|}
\hline thickness & above & 2.2 & $16.70 \mathrm{gr}$. & \multicolumn{2}{|c|}{8 grains } \\
\hline "I & $\prime \prime$ & 2.0 & $40.80 \prime \prime$ & 25 & $\prime \prime$ \\
\hline " & $\because \prime$ & 1.8 & $31.20 \prime \prime$ & 16 & $" \prime$ \\
\hline " & under & $\begin{array}{r}1.8 \\
\text { average. }\end{array}$ & $\begin{array}{c}2.20 " ~ \\
\max .\end{array}$ & $\begin{array}{c}1 \\
\min .\end{array}$ & $\prime \prime$ \\
\hline & length & 4.90 & 5.50 & 4.40 & \\
\hline & width & 2.77 & 3.10 & 2.40 & \\
\hline
\end{tabular}

d) Percentage of Shinziromai.- $0.4 \%$

e) Depth of longitudinal furrow. $(\mu)$

$\begin{array}{rlr}\text { average. } & \max . & \min . \\ 40.48 & 51.90 & 25.95\end{array}$

f) Specific gravity.--1.394

g) Saccharifying quality. $-39.49 \%$

h) Hardness. (kilo)

\begin{tabular}{|c|c|c|c|}
\hline \multirow{2}{*}{\multicolumn{2}{|c|}{$\begin{array}{l}\text { under } 3.75 \text { kilo } \\
3.75-5.63\end{array}$}} & \multicolumn{2}{|c|}{5 grains. } \\
\hline & & 27 & $\prime \prime$ \\
\hline $5.63-7.50$ & & 18 & " \\
\hline $7.50-9.38$ & & 一 & \\
\hline above 9.38 & & - & \\
\hline $\begin{array}{c}\text { average. } \\
5.2695\end{array}$ & $\begin{array}{l}\max . \\
7.275\end{array}$ & & ain. \\
\hline
\end{tabular}

i) Volume of $100 \mathrm{~g}$. - $128.5 \mathrm{c} . \mathrm{c}$.

j) Weight of 1,000 grains $-21.60 \mathrm{~g}$.

m) Thickness of bran layer. $(\mu)$

$\begin{array}{crr}\text { average. } & \max . & \min . \\ 44.64 & 56.25 & 33.75\end{array}$

n) Absorption of water.

$\begin{array}{ccc}\text { time steeped. } & \text { water absorbed. } & \% \text { absorbed. } \\ 1 \text { h. } & 3.95 & 7.90 \\ 3 & 7.80 & 15.60 \\ 24 & 10.95 & 21.90\end{array}$

o) Depth of embryo cavity.
average.
$\max$.
$\min$.
0.28
0.33
0.25

p) Chemical composition.-

Water

D. R. sugar

Dextrin

Starch

Crude fat

" fibre unbydrous.
3.41
2.77
81.14
0.86
1.17 


$\begin{array}{lll}\text { " protein } & 7.41 & 8.85 \\ \text { Ash } & 1.37 & 1.64 \\ \text { Phoshoric acid } & 0.43 & 0.52\end{array}$

R) Production of Yamagata prefecture.

a) Percentage of full grown grains. $-85.8 \%$

b) External appearance and luster.- Straw yellow waxy luster, grains were fat and small, impurities were tolerably abundant.

c) Size of grains. (m.m.)

\begin{tabular}{|c|c|c|c|c|c|}
\hline thickness & above & 2.3 & $450 \mathrm{gr}$. & \multicolumn{2}{|c|}{23 grains. } \\
\hline " & " & \multirow{2}{*}{$\begin{array}{l}2.0 \\
1.8\end{array}$} & $38.2 \prime \prime$ & 19 & $" \prime$ \\
\hline$\prime \prime$ & 11 & & $14.4 " \prime$ & 7 & $\prime \prime$ \\
\hline$\prime \prime$ & under & 1.8 & $2.2 " \prime$ & 1 & $"$ \\
\hline & gth & $\begin{array}{c}\text { average. } \\
4.89\end{array}$ & $\begin{array}{r}\max . \\
5.40\end{array}$ & $\begin{array}{r}\text { min. } \\
4.20\end{array}$ & \\
\hline \multicolumn{2}{|c|}{ width } & 2.79 & 3.00 & 2.60 & \\
\hline
\end{tabular}

d) Percentage of Shinziromai.-- $2.8 \%$

e) Depth of longitudinal furrow. $(\mu)$

$\begin{array}{rcr}\text { average. } & \max . & \min . \\ 47.49 & 69.20 & 17.30\end{array}$

f) Specific gravity.-1.389

g) Saccharifying quality. - $39.23 \%$

h) Hardness. (kilo)

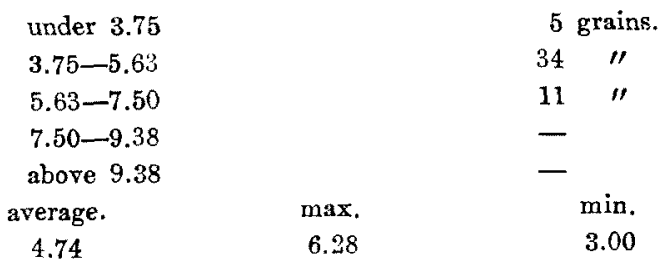

i) Volume of $100 \mathrm{~g} . \longrightarrow 128.5 \mathrm{c} . \mathrm{c}$.

j) Weight of 1,000 grains._-21.9g.

m) Thickness of bran layer. $(\mu)$

$\begin{array}{rrr}\text { average. } & \max . & \min . \\ 44.97 & 67.50 & 36.0\end{array}$

n) Absorption of water.

$\begin{array}{ccc}\text { time steeped. } & \text { water absorbed. } & \% \text { absorbed } \\ 1 \text { h. } & 2.78 & 5.56 \\ 3 & 5.30 & 10.60 \\ 24 & 9.90 & 19.80\end{array}$

o) Depth of embryo cavity.

$\begin{array}{ccc}\text { average. } & \max & \min . \\ 0.27 & 0.29 & 0.25\end{array}$

p) Chemical composition.- 


$\begin{array}{lrc} & & \text { unhydrous. } \\ \text { Water } & 14.89 & - \\ \text { D. R. sugar } & 3.12 & 3.67 \\ \text { Dextrin } & 3.00 & 3.52 \\ \text { Starch } & 69.72 & 81.92 \\ \text { Crude fat } & 1.07 & 1.25 \\ \quad \text { " fibre } & 0.84 & 0.99 \\ \quad \text { " protein } & 7.06 & 8.29 \\ \text { Ash } & 1.20 & 1.41 \\ \text { Phosphoric acid } & 0.37 & 0.43\end{array}$

\section{Production.}

a) Husked rice unsuitable for the Sake brewing. 4 (I-IV) varieties.

I) Production of Fukui prefecture.

a) Percentage of full grown grains. $92.7 \%$

b) External appearance and luster.- Straw yellow waxy luster, grains were large and fat.

c) Size of grains. (m.m.)

\begin{tabular}{|c|c|c|c|c|c|}
\hline thicknes & above & 2.2 & $69.70 \mathrm{~g}$. & \multicolumn{2}{|c|}{35 grains. } \\
\hline$\prime \prime$ & $\prime \prime$ & 2.0 & $24.70 \prime \prime$ & 12 & $\prime \prime$ \\
\hline$\prime \prime$ & "I & 1.8 & $5.10 " 1$ & 3 & $\prime \prime$ \\
\hline$\prime \prime$ & under & 1.8 & $0.30 \prime \prime$ & - & \\
\hline & & average. & max. & min. & \\
\hline & gth & 5.25 & 5.60 & 4.80 & \\
\hline & dth & 3.01 & 3.20 & 2.60 & \\
\hline
\end{tabular}

d) Percentage of Shinziromai._- $74.0 \%$

e) Depth of longitudinal furrow. $(\mu)$

$\begin{array}{rrr}\text { average. } & \max . & \min . \\ 52.94 & 86.50 & 29.41\end{array}$

f) Specific gravity.-- 1.404 .

g) Saccharifying quality.—37.95\%

h) Hardness. (kilo)

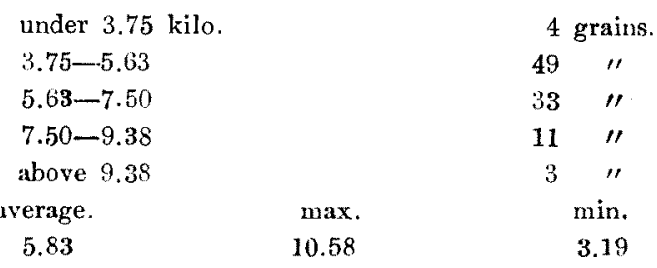

i) Volume of $100 \mathrm{~g}$. $122.0 \mathrm{c} . \mathrm{c}$.

j) Weight of 1,000 grains. $-26.10 \mathrm{~g}$.

$\mathrm{m})$ Thickness of bran layer. $(\mu)$

$\begin{array}{rrr}\text { average. } & \max . & \min . \\ 52.49 & 72.0 & 33.75\end{array}$


n) Absorption of water.

$\begin{array}{ccc}\text { time steeped. } & \text { water absorbed. } & \% \text { absorbed. } \\ 1 \mathrm{~h} . & 2.40 & 4.80 \\ 3 & 5.45 & 10.90 \\ 24 & 11.85 & 23.70\end{array}$

o) Depth of embryo cavity.

$\begin{array}{ccc}\text { average. } & \max . & \min . \\ 0.23 & 0.28 & 0.19\end{array}$

p) Chemical compositions.-

$\begin{array}{lrr}\text { Water } & 12.56 & - \\ \text { D. R. sugar } & 2.40 & 2.74 \\ \text { Dextrin } & 2.43 & 2.77 \\ \text { Stareh } & 70.65 & 80.80 \\ \text { Crude fat } & 2.23 & 2.55 \\ \quad \text { " fibre } & 1.04 & 1.18 \\ \quad \text { " protein } & 6.70 & 7.66 \\ \text { Ash } & 1.26 & 1.44 \\ \text { Phosphoric } & 0.58 & 0.66\end{array}$

II) Production of Shiga prefecture.

a) Percentage of full grown grains.-- $81.65 \%$

b) External appearance and luster.- Straw brown yellow colour, no luster, grains were fat and middle size, impurities were tolerably abundant.

c) Size of grains. (m.m.)

\begin{tabular}{|c|c|c|c|c|c|}
\hline thickness & above. & 2.2 & $42.70 \mathrm{~g}$. & 21 & rains \\
\hline$\prime \prime$ & $" \prime$ & 2.0 & $38.10 "$ & 19 & $" \prime$ \\
\hline " & " & 1.8 & $16.80 "$ & 9 & " \\
\hline " & under & 1.8 & $2.2 \quad 11$ & 1 & " \\
\hline & & average. & $\max$ & $\min$. & \\
\hline & igth & 5.10 & 5.50 & 4.50 & \\
\hline & dth & 2.92 & 3.10 & 2.50 & \\
\hline
\end{tabular}

d) Percentage of Shinziromai.- $8.4 \%$

e) Depth of longitudinal furrow. $(\mu)$

$\begin{array}{rcr}\text { average. } & \max . & \text { min. } \\ 51.97 & 91.69 & 34.60\end{array}$

f) Specific gravity.-1.404.

g) Saccharifying quality.-31.65\%

h) Hardness. (kilo)

under 3.75
$3.75-5.63$
$5.63-7.50$
$7.50-9.38$
above 9.38
average.
7.05


i) Volume of $100 \mathrm{~g}$. $125.0 \mathrm{c} . \mathrm{c}$.

j) Weight of 1,000 grains. $-23.75 \mathrm{~g}$.

m) Thickness of bran layer. $(\mu)$

$\begin{array}{ccc}\text { nverage. } & \max . & \min . \\ 56.23 & 67.50 & 38.70\end{array}$

n) Absorption of water.

$\begin{array}{ccc}\text { time steeped. } & \text { water absorbed. } & \% \text { absorbed. } \\ 1 \mathrm{~h} . & 3.000 & 6.00 \\ 3 & 6.450 & 1290 \\ 24 & 12.420 & 24.84\end{array}$

o) Depth of embryo cavity.

$\begin{array}{ccc}\text { average. } & \max . & \min \\ 0.22 & 0.29 & 0.13\end{array}$

p) Chemical composition.-

$\begin{array}{lrr}\text { Water } & 12.14 & - \\ \text { D. R. sugar } & 6.70 & 0.80 \\ \text { Dextrin } & 0.99 & 1.12 \\ \text { Starch } & 74.56 & 84.86 \\ \text { Crude fat } & 2.23 & 2.54 \\ \quad \text { " fibre } & 1.13 & 1.29 \\ \quad \text { " protein } & 7.15 & 8.14 \\ \text { Ash } & 1.45 & 1.65 \\ \text { Phosphoric acid } & 0.37 & 0.42\end{array}$

III) Production of Ishikawa prefecture.

a) Percentage of full grown grains,- $-90.0 \%$.

b) External appearance and luster.-Dense straw yellow colour, no luster, grain were fat and middle size, impurities were tolerably abundant.

c) Size of grains. (m.m.)

\begin{tabular}{|c|c|c|c|c|c|}
\hline thickness & above & 22 & $79.10 \mathrm{~g}$. & \multicolumn{2}{|c|}{40 grains. } \\
\hline$" \prime$ & $\prime \prime$ & 2.0 & $16.30 "$ & 8 & $\prime \prime$ \\
\hline$"$ & $" \prime$ & 1.8 & $3.70 \prime$ & 2 & $" \prime$ \\
\hline$\prime \prime$ & under & 1.8 & $0.70 " \prime$ & - & \\
\hline & ingth & $\begin{array}{c}\text { average. } \\
5.16\end{array}$ & $\begin{array}{c}\max . \\
5.50\end{array}$ & $\begin{array}{l}\text { min. } \\
4.70\end{array}$ & \\
\hline & dth & 2.92 & 3.20 & 2.60 & \\
\hline
\end{tabular}

d) Percentage of Shinziromai.-2.2\%

e) Depth of longitudinal furrow. $(\mu)$

$\begin{array}{ccc}\text { average. } & \max . & \min . \\ 56.48 & 86.50 & \mathbf{3 4 . 6 0}\end{array}$

f) Specific gravity.- -1.399

g) Saccharifying quality. $-43.49 \%$.

h) Hardness. (kilo) 
under. 3.75

$3.75-5.63$

$5.63-7.50$

$7.50-9.38$

above 9.38

average.

6.82
- grains.

31

37

23

9

min.

3.84

i) Volume of $100 \mathrm{~g}$. - 125.5 c.c.

j) Weight of 1,000 grains. $-25.35 \mathrm{~g}$.

m) Thickness of bran layer. $(\mu)$ average.

$\max$.

$\min$.

58.43

94.50

34.65

n) Absorption of water.

$\begin{array}{ccc}\text { time steeped. } & \text { water absorbed. } & \% \text { abosorbed } \\ 1 \text { h. } & 3.12 & 6.24 \\ 3 & 6.75 & 13.50 \\ 24 & 12.40 & 24.80\end{array}$

o) Depth of embryo cavity.

$\begin{array}{ccc}\text { average. } & \max . & \min . \\ 0.24 & 0.27 & 0.18\end{array}$

p) Chemical composition.-

$\begin{array}{lrr}\text { Water } & 12.05 & - \\ \text { D. R. sugar } & 3.86 & 3.26 \\ \text { Dextrin } & 0.70 & 0.79 \\ \text { Starch } & 73.04 & 83.05 \\ \text { Crude fat } & 2.47 & \mathbf{2 . 8 1} \\ \quad \text { " fibre } & 1.16 & 1.32 \\ \quad \text { " protein } & 7.15 & 8.13 \\ \text { Ash } & 1.20 & 1.36 \\ \text { Phosphoric acid } & 0.34 & 0.38\end{array}$

IV) Production of Shizuoka prefecture.

a) Percentage of full grown grains._- $82.1 \%$

b) External appearance and luster.-Greyish brown straw yellowish colour, no luster, grains were not fat and middle size, impurities abundant.

c) Size of grains. (m.m.)

\begin{tabular}{|c|c|c|c|c|c|}
\hline thickness & abore & 2.2 & $46.90 \mathrm{~g}$. & 24 & grains \\
\hline$\prime \prime$ & $\mu$ & 2.0 & $36.10 \prime \prime$ & 18 & " \\
\hline$\prime \prime$ & " & 1.8 & $14.20 \mathrm{ll}$ & 7 & " \\
\hline$\prime \prime$ & under & $\begin{array}{c}1.8 \\
\text { average. }\end{array}$ & $\begin{array}{l}2.50 " \prime \\
\text { max. }\end{array}$ & $\begin{array}{c}1 \\
\text { min. }\end{array}$ & " \\
\hline & gth & 5.11 & 5.60 & 4.50 & \\
\hline & ath & 2.94 & 3.20 & 2.60 & \\
\hline
\end{tabular}

d) Percentage of Shinziromai.-18.8\%

e) Depth of longitudinal furrow. $(\mu)$ 


$\begin{array}{crr}\text { average. } & \max . & \text { min. } \\ 54.51 & 87.50 & 17.50\end{array}$

f) Specific gravity.- -1.399

g) Saccharifying quality.- $-43.22 \%$

h) Hardness. (kilo)

\begin{tabular}{ccc} 
under 3.75 & & \multicolumn{2}{c}{1 grains. } \\
$3.75-5.63$ & $36 " \prime \prime$ \\
$5.63-7.50$ & & $49 " \prime$ \\
$7.50-9.38$ & $13 " \prime \prime$ \\
above 9.38 & & $1 \prime \prime$ \\
average. & $\max$. & $\mathrm{min}$. \\
6.03 & 9.56 & 3.69
\end{tabular}

i) Volume of $100 \mathrm{~g}$ - $128.0 \mathrm{c} . \mathrm{c}$.

j) Weight of 1,000 grains. $24.0 \mathrm{~g}$.

$\mathrm{m})$ Thickness of bran layer. $(\mu)$

$\begin{array}{rrr}\text { average. } & \max . & \text { min. } \\ 48.16 & 60.75 & 37.35\end{array}$

n) Absorption of water.

$\begin{array}{ccc}\text { time steeped. } & \text { water absorbed. } & \% \text { absorbed. } \\ 1 \mathrm{~h} . & 3.05 & 6.1 \\ 3 & 6.00 & 12.0 \\ 24 & 11.15 & 22.30\end{array}$

o) Depth of embryo cavity.

$\begin{array}{ccc}\text { average. } & \max . & \mathrm{min} . \\ 0.25 & 0.30 & 0.20\end{array}$

p) Chemical composition.-

$\begin{array}{lrr}\text { Water } & 13.73 & - \\ \text { D. R. sugar } & 2.13 & 2.47 \\ \text { Dextrin } & 1.74 & 2.01 \\ \text { Starch } & 69.72 & 80.71 \\ \text { Crude fat } & 2.06 & 239 \\ \quad \text { " fibre } & 1.19 & 1.38 \\ \quad \text { " protein } & 8.22 & 9.52 \\ \text { Ash } & 1.26 & 1.46 \\ \text { Phosphoric acid } & 0.97 & 0.43\end{array}$

1916 Production.

B) Polished rice, suitable for the Saké brewing. $6(\mathrm{~A}-\mathrm{F})$ varieties.

A) Production of Okayama prefecture.

a) Percentage of full grown grains.- $84.5 \%$

b) External appearance and luster.-White glassy luster, grains were large and fat, broken grains were abundant.

c) Size of grains. (m.m.) 


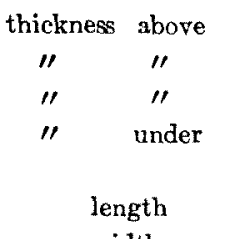

width

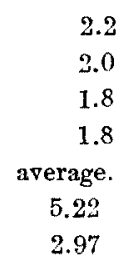

$35.5 \mathrm{~g}$.

$49.2 \mathrm{ll}$

$18.2 \prime \prime$

$2.8 " 1$

$\max$.

5.50

3.15 \begin{tabular}{rr} 
& \multicolumn{2}{c}{ grains. } \\
22 & $\prime \prime$ \\
9 & $\prime \prime$ \\
1 & $\prime \prime$ \\
min. & \\
4.8 & \\
2.1 &
\end{tabular}

min.

17.3

f) Specific gravity.--1.439

g) Saccharifying quality. $-33.28 \%$

h) Hardness. (kilo)

\begin{tabular}{|c|c|c|}
\hline under 3.75 & & 41 grains \\
\hline $3.75-5.63$ & & $38 \quad \prime \prime$ \\
\hline $5.63-7.50$ & & 19 \\
\hline $7.50-9.38$ & & $"$ \\
\hline above 9.38 & & - \\
\hline verage. & $\max$. & $\min$. \\
\hline
\end{tabular}

i) Volume of $100 \mathrm{~g}$.—117.5c.c.

j) Weight of 1,000 grains.—-23.8g.

n) Absorption of water.

$\begin{array}{ccc}\text { time steeped. } & \text { water absorbed. } & \% \text { absorbed. } \\ 30 \mathrm{~m} . & 10.30 \mathrm{~g} . & 20.60 \\ 2 \mathrm{~h} . & 11.87 \prime \prime & 23.74\end{array}$

p) Chemical composition :-

$\begin{array}{lrr}\text { Water } & 13.31 & - \\ \text { D. R. sugar } & 1.07 & 1.24 \\ \text { Dextrin } & 3.07 & 3.54 \\ \text { Starch } & 76.61 & 88.37 \\ \text { Crude fat } & 0.28 & 0.33 \\ \quad \text { " fibre } & 0.49 & 0.57 \\ \quad \text { " protein } & 5.98 & 6.89 \\ \text { Ash } & 0.36 & 0.42 \\ \text { Phosphoric acid } & 0.13 & 0.15\end{array}$

B) Production of Okayama prefecture.

a) Percentage of full grown grains.- $93.54 \%$

b) External appearance and luster- Same as before.

c) Size of grains. (m.m.)

\begin{tabular}{|c|c|c|c|c|c|c|}
\hline thickness & above & 2.2 & 50.90 & & 25 & rains. \\
\hline$\prime \prime$ & $\prime \prime$ & 2.0 & 38.8 & $" \prime$ & 19 & $\prime \prime$ \\
\hline$\prime \prime$ & " & 1.8 & 9.0 & $\prime \prime$ & 5 & $\prime \prime$ \\
\hline$\prime \prime$ & under & 1.8 & 1.0 & $\prime \prime$ & 1 & $" \prime$ \\
\hline
\end{tabular}




$\begin{array}{lccc} & \text { average. } & \max . & \min . \\ \text { length } & 5.16 & 5.50 & 4.40 \\ \text { width } & 2.92 & 3.10 & 2.50\end{array}$

d) Percentage of Shinziromai.- $69.5 \%$

e) Depth of longitudinal furrow. $(\mu)$

$\begin{array}{ccr}\text { average. } & \max . & \min . \\ 32.87 & 51.90 & 17.30\end{array}$

f) Specific gravity. -1.444

g) Saccharifying quality.- $31.58 \%$

h) Hardness. (kilo)

\begin{tabular}{|c|c|c|}
\hline under 3.75 & & 54 \\
\hline $3.75-5.63$ & & 39 \\
\hline $5.63-7.50$ & & 7 \\
\hline $7.50-9.38$ & & 一 \\
\hline above 6.38 & & - \\
\hline $\begin{array}{c}\text { iverage. } \\
3.80\end{array}$ & $\begin{array}{l}\max . \\
6.30\end{array}$ & \\
\hline
\end{tabular}

i) Volume of $100 \mathrm{~g} . \longrightarrow 118.0$ c.c.

j) Weight of 1,000 grains. $-24.5 \mathrm{~g}$.

n) Absorption of water.

$\begin{array}{ccc}\text { time steeped. } & \text { water absorbed. } & \% \text { absorbed. } \\ 30 \mathrm{~m} . & 10.70 \mathrm{~g} . & 21.4 \\ 2 \mathrm{~h} . & 11.65 \mathrm{ll} & 23.3\end{array}$

p) Chemical composition :

$\begin{array}{lrr}\text { Water } & 12.71 & - \\ \text { D. R. sugar } & 0.77 & 0.88 \\ \text { Dextrin } & 2.99 & 3.42 \\ \text { Starch } & 76.64 & 87.79 \\ \text { Crude fat } & 0.30 & 0.35 \\ \quad \text { " fibre } & 0.49 & 0.56 \\ \quad \text { " protein } & 5.98 & 6.85 \\ \text { Ash } & 0.38 & 0.44 \\ \text { Phosphoric acid } & 0.11 & 0.12\end{array}$

C) Production of Okayama prefecture.

a) Percentage of full grown grains.- $92.65 \%$

b) External appearance and luster.-Same as before.

c) Size of grains. (m.m.)

\begin{tabular}{|c|c|c|c|c|c|}
\hline thicknes. & above & 3.2 & $47.10 \mathrm{~g}$. & \multicolumn{2}{|c|}{24 grains } \\
\hline$" \prime$ & $" \prime$ & 3.0 & $40.10 " \prime$ & 20 & $" \prime$ \\
\hline$" \prime$ & $" \prime$ & 1.8 & 11.011 & 5 & $" \prime$ \\
\hline$"$ & under & $\begin{array}{c}1.8 \\
\text { average. }\end{array}$ & $\begin{array}{l}11.4 \quad " \prime \\
\max .\end{array}$ & $\begin{array}{c}1 \\
\min .\end{array}$ & " \\
\hline & gth & 5.02 & 5.60 & 4.6 & \\
\hline & th & 9.92 & 3.10 & 2.5 & \\
\hline
\end{tabular}


d) Percentage of Shinziromai._-61.80\%

e) Depth of longitudinal furrow. $(\mu)$

$\begin{array}{ccc}\text { average. } & \max . & \min . \\ 34.77 & 46.71 & 17.30\end{array}$

f) Specific gravity.-1.439\%

17.30

g) Saccharifying quality. $-26.50 \%$

h) Hardness. (kilo)

$$
\begin{aligned}
& \text { under } 3.75 \\
& 3.75-5.63 \\
& 5.63-7.50 \\
& 7.50-9.38 \\
& \text { above } 9.38 \\
& \text { average. }
\end{aligned}
$$

3.85

i) Volume of $100 \mathrm{~g}$

$\max$.

7.03

$118.0 \mathrm{c} . \mathrm{c}$.

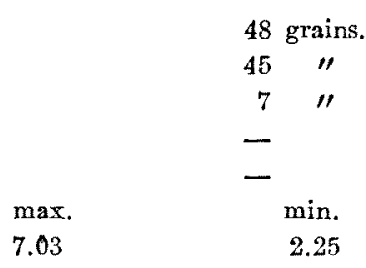

j) Weight of 1,000 grains.-24.0g.

n) Absorption of water. time steeped. $30 \mathrm{~m}$. water absorbed. $9.40 \mathrm{~g}$. $10.95 \prime \prime$

p) Chemical composition :-

$\begin{array}{lr}\text { Water } & 13.89 \\ \text { D. R. sugar } & 0.85 \\ \text { Dextrin } & 2.04 \\ \text { Starch } & 76.68 \\ \text { Crude fat } & 0.39 \\ \quad \text { " fibre } & 0.57 \\ \quad \text { " protein } & 5.81 \\ \text { Ash } & 0.42 \\ \text { Phosphoric acid } & 0.16 \\ \text { of Miye prefecture. } & \end{array}$

13.89

6.68

0.39

0.57

0.42

16
D) Producition of Miye prefecture.

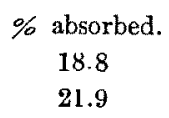

\begin{tabular}{|c|c|c|c|c|c|}
\hline \multicolumn{2}{|c|}{ thickness above } & \multirow{2}{*}{$\begin{array}{l}2.2 \\
2.0\end{array}$} & \multirow{2}{*}{$\begin{array}{l}14.90 \mathrm{~g} . \\
38.50 \mathrm{\prime \prime}\end{array}$} & \multicolumn{2}{|c|}{8 grains. } \\
\hline$\prime \prime$ & $\prime \prime$ & & & 20 & $\prime \prime$ \\
\hline$" \prime$ & $\prime \prime$ & 1.8 & $36.20 "$ & 18 & $\prime \prime$ \\
\hline$" 1$ & $\prime \prime$ & 1.6 & $6.30 \prime \prime$ & 3 & $" \prime$ \\
\hline$"$ & under & 1.6 & $2.30 \prime \prime$ & 1 & $"$ \\
\hline & & average. & $\max$. & min. & \\
\hline & gth & 5.08 & 5.60 & 4.6 & \\
\hline & Ith & 2.82 & 3.00 & 2.4 & \\
\hline
\end{tabular}

unhydrous.

a) Percentage of full grown grains.- $84.6 \%$

b) External appearance and luster. Slightly yellowish white glassy luster, grains were fat and middle size, broken grains were perceived.

c) Size of grains. (m.m.)

d) Percentage of Shinziromai.- $53.6 \%$

e) Depth of longitudinal furrow. $(\mu)$ 


$\begin{array}{ccr}\text { average, } & \max . & \min . \\ \mathbf{3 8 . 9 3} & 64.01 & \mathbf{2 5 . 9 5}\end{array}$

f) Specific gravity.-1.439

g) Saccharifying quality.- $-30.73 \%$

h) Hardness. (kilo)

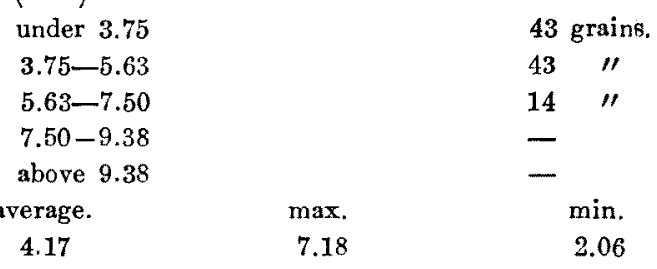

i) Volume of $100 \mathrm{~g} .-123.0$ c.c.

j) Weight of 1,000 grains. $-21.7 \mathrm{~g}$.

n) Absorption of water.

$\begin{array}{ccc}\text { time steeped. } & \text { water absorbed. } & \% \text { absorbed. } \\ 30 \mathrm{~m} & 8.10 \mathrm{gr} . & 16.2 \\ 2 \mathrm{~h} & 10.4 \prime \prime & 20.8\end{array}$

p) Chemical composition :-

$\begin{array}{lrr}\text { Water } & 13.24 & - \\ \text { D. R. sugar } & \mathbf{1 . 6 2} & 1.86 \\ \text { Dextrin } & 3.02 & 3.49 \\ \text { Starch } & 73.82 & 85.09 \\ \text { Crude fat } & 0.60 & 0.69 \\ \quad \text { " fibre } & 0.66 & 0.76 \\ \quad \text { " protein } & 5.98 & 6.89 \\ \text { Ash } & 0.74 & 0.85 \\ \text { Phosphoric acid } & 0.14 & 0.16\end{array}$

E) Production of Niigata prefecture.

a) Percentage of full grown grains.-94.45\%

b) External appearance and luster.- Nearly same as before.

c) Size of grains. (m.m.)

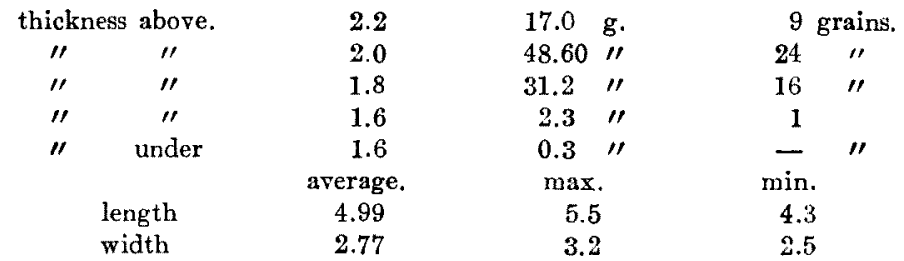

d) Percentage of Shinziromai.—-14.4\%

e) Depth of longitudinal furrow. $(\mu)$ $\begin{array}{rrr}\text { average. } & \max . & \min . \\ 41.87 & 59.20 & 25.95\end{array}$

f) Specific gravity.- 1.434

g) Saccharifying quality. $-37.69 \%$ 
h) Hardness. (kilo)

\begin{tabular}{|c|c|c|}
\hline under 3.75 & & \\
\hline $3.75-5.63$ & & 56 \\
\hline $5.63-7.50$ & & 32 \\
\hline $7.5^{\circ} 0-9.38$ & & 1 \\
\hline above 9.38 & & - \\
\hline $\begin{array}{c}\text { average. } \\
5.00\end{array}$ & $\max$. & \\
\hline
\end{tabular}

i) Volume of $100 \mathrm{~g}$. $122.5 \mathrm{c} . \mathrm{c}$.

j) Weight of 1,000 grains. $-22.1 \mathrm{~g}$.

n) Absorption of water.

$\begin{array}{ccc}\text { time steeped. } & \text { water absorbed. } & \% \text { absorbed, } \\ 30 \mathrm{~m} & 8.58 \mathrm{gr} . & 17.16 \\ 2 \mathrm{~h} & 9.58 \mathrm{ll} & 19.16\end{array}$

p) Chemical composition :-

$\begin{array}{lrr}\text { Water } & 13.56 & \text { unhydrous. } \\ \text { D. R. sugar } & 0.66 & - \\ \text { Dextrin } & 1.71 & 0.77 \\ \text { Starch } & 75.73 & 1.97 \\ \text { Crude fat } & 0.17 & 87.60 \\ \quad \text { ' fibre } & 0.27 & 0.19 \\ \quad \prime \quad \text { protein } & 6.88 & 0.31 \\ \text { Ash } & 0.49 & 7.96 \\ \text { Phosphoric acid } & 0.12 & 0.57 \\ \end{array}$

F) Production of Miyagi prefecture.

a) Percentage of full grown grains.-93.8\%

b) External appearance and luster.-Nearly same as before.

c) Size of grains. (m.m.)

\begin{tabular}{|c|c|c|c|c|c|}
\hline bick & above & 2.2 & $60.70 \mathrm{~g}$ & 31 & rains \\
\hline$" \prime$ & $\prime \prime$ & 2.0 & $25.80 \prime \prime$ & 13 & $\prime \prime$ \\
\hline$\prime \prime$ & "' & 1.8 & $10.70 \mathrm{\prime \prime}$ & 5 & " \\
\hline "I & under & $\begin{array}{c}1.8 \\
\text { average. }\end{array}$ & $\begin{array}{l}2.20 " \prime \\
\max .\end{array}$ & $\begin{array}{c}1 \\
\text { min. }\end{array}$ & " \\
\hline & agth & 5.03 & 5.55 & 4.60 & \\
\hline & dth & 3.06 & 3.30 & 2.85 & \\
\hline
\end{tabular}

d) Percentage of Shinziromai._- $10.4 \%$

e) Depth of longitudinal furrow. $(\mu)$

$\begin{array}{rrr}\text { average. } & \max . & \min . \\ 45.07 & 64.01 & 25.95\end{array}$

f) Specific gravity. -1.434

g) Saccharifying quality.-34.01\%

h) Hardness. (kilo)

$\begin{array}{lc}\text { under } 3.75 & 4 \\ 3.75-5.63 & 36 " \prime \prime \\ 5.63-7.50 & 10 " \prime \prime\end{array}$




$$
\begin{gathered}
7.50-9.38 \\
\text { above } 9.38
\end{gathered}
$$

average.

4.84 $\max$.

6.84 min.

2.81

i) Volume of $100 \mathrm{~g}$ - $125.0 \mathrm{c} . \mathrm{c}$.

j) Weight of 1,000 grains. $-22.8 \mathrm{~g}$.

n) Absorption of water.

$\begin{array}{ccc}\text { time steeped. } & \text { water absorbed. } & \% \text { absorbed. } \\ 30 \mathrm{~m} . & 4.98 \mathrm{~g} . & 996 \\ 2 \mathrm{~h} . & 8.98 \% & 17.36\end{array}$

p) Chemical composition :-

$\begin{array}{lrr}\text { Water } & \mathbf{1 3 . 8 2} & - \\ \text { D. R. sugar } & 1.34 & \mathbf{1 . 5 6} \\ \text { Dextrin } & 2.62 & 3.04 \\ \text { Starch } & 75.39 & 87.47 \\ \text { Crude fat } & 0.38 & 0.45 \\ \quad \text { " fibre } & 0.21 & 0.21 \\ \quad \text { " protein } & 7.60 & 8.81 \\ \text { Ash } & 0.77 & 0.89 \\ \text { Phosphoric acid } & 0.16 & 0.19\end{array}$

\section{Production.}

$\left.\beta^{\prime}\right)$ Polished rice, unsuitable for the Sake brewing. 6 (I-VI) varieties.

I) Production of Hiroshima prefecture.

a) Percentage of full grown grains._- $91.3 \%$

b) External appearance and luster.—Slightly greyish white colour, grains were fat and middle size.

c) Size of grains. (m.m.)

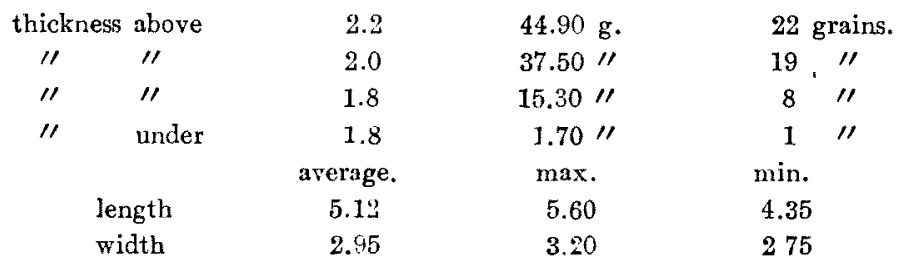

d) Percentage of Shinziromai.-41.2\%

e) Depth of longitudinal furrow. $(\mu)$

$$
\begin{array}{rrr}
\text { average. } & \max . & \min . \\
36.07 & 51.90 & 17.30
\end{array}
$$

f) Specific gravity.-- -1.434

g) Saccharifying quality.-36.78\%

h) Hardness. (kilo) 

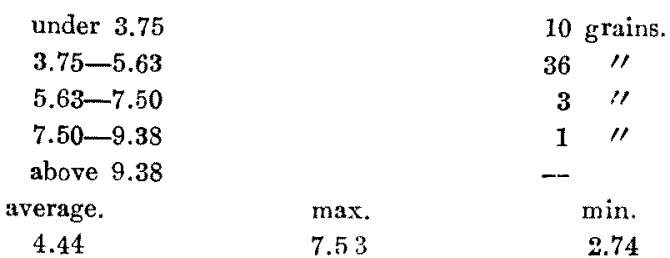

i) Volume of $100 \mathrm{~g} . \longrightarrow 124.0 \mathrm{c} . \mathrm{c}$.

j) Weight of 1,000 grains. $-22.3 \mathrm{~g}$.

n) Absorption of water.

$\begin{array}{ccc}\text { time steeped. } & \text { water absorbed. } & \% \text { absorbed. } \\ 30 \mathrm{~m} & 8.13 \mathrm{~g} . & 16.26 \\ 2 \mathrm{~h} & 10.05 \% & 20.10\end{array}$

p) Chemical composition :-

$\begin{array}{lrc}\text { Water } & 13.36 & - \\ \text { D. R. sugar, } & 0.57 & 0.65 \\ \text { Dextrin } & 1.52 & 1.76 \\ \text { Starch } & 77.35 & 89.27 \\ \text { Crude fat } & 0.37 & 0.42 \\ \quad \prime \text { fibre } & 0.40 & 0.46 \\ \quad \prime \text { protein } & 6.34 & 7.32 \\ \text { Ash } & 0.68 & 0.78 \\ \text { Phosphoric acid } & 0.15 & 0.17\end{array}$

II) Production of Slizwoka prefecture.

a) Percentage of full grown grains.- $85.65 \%$

b) External appearance and luster.-Nearly same as before.

c) Size of grains. (m.m.)

\begin{tabular}{|c|c|c|c|c|c|}
\hline thick & above & 2.2 & $25.0 \mathrm{~g}$. & 13 & rains. \\
\hline$\prime \prime$ & 11 & 2.0 & $45.9 \prime \prime$ & 23 & 11 \\
\hline " & $\prime \prime$ & 1.8 & $25.7 " \prime \prime$ & 13 & $\prime \prime$ \\
\hline "I & 11 & 1.6 & 2.511 & 1 & $\prime \prime$ \\
\hline "I & under & 1.6 & $0.5 \prime \prime$ & - & \\
\hline & & average. & $\max$. & min. & \\
\hline & ngth & 4.93 & 5.40 & 4.20 & \\
\hline & idth & 2.84 & 3.10 & 2.30 & \\
\hline
\end{tabular}

d) Percentage of Shinziromai,-23.2\%

e) Depth of longitudinal furrow. $(\mu)$

$\begin{array}{ccc}\text { average. } & \max . & \mathrm{min} . \\ 41.69 & 69.20 & 8.65\end{array}$

f) Specific gravity.-1.439

g) Saccharifying quality. -31.85

h) Hardness. (kilo)

under 3.75 


$$
\begin{aligned}
& 5.63-7.50 \\
& 7.50-9.38 \\
& \text { above } 9.38 \\
& \text { average. } \\
& 4.63
\end{aligned}
$$

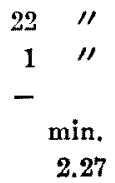

$\max$.

7.93

i) Volume of $100 \mathrm{~g}$. - 125.0c.c.

j) Weight of 1,000 grains.- $21.7 \mathrm{~g}$.

n) Absorption of water.

$\begin{array}{ccc}\text { time steeped. } & \text { water absorbed. } & \% \text { absorbed. } \\ 30 \mathrm{~m} . & 6.22 \mathrm{~g} . & 12.44 \\ 2 \mathrm{~h} . & 9.86 / \prime & 19.70\end{array}$

p) Chemical composition :-

$\begin{array}{lrr}\text { Water } & 13.21 & - \\ \text { D. R. sugar } & 1.50 & 1.73 \\ \text { Dextrin } & \mathbf{3 . 4 3} & 3.96 \\ \text { Starch } & 72.12 & 83.10 \\ \text { Crude fat } & 0.77 & 0.89 \\ \quad \text { " fibre } & 0.39 & 0.45 \\ \quad \text { " protein } & 7.86 & 9.05 \\ \text { Ash } & 0.85 & 0.87 \\ \text { Phosphoric acid } & 0.18 & 0.21\end{array}$

III) Production of Kagawa prefecture.

a) Percentage of full grains. $-94.4 \%$

b) External appearance and luster.-Brownish yellow colour, grains were not fat and middle size.

c) Size of grains. (m.m.)

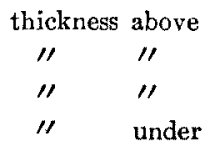

length

width
2.2

2.0

1.8

1.8

average.

4.83

2.72
$10.40 \mathrm{~g}$.

47.60 "l

$37.20 " 1$

$4.50 " \prime$

$\max$.

5.30

2.90
5 grains.

$24 \quad "$

1911

211

min.

4.30

2.50

d) Percentage of Shinziromai.-4.8\%

e) Depth of longitudinal furrow. $(\mu)$

$$
\begin{array}{rlr}
\text { average. } & \max . & \min . \\
38.41 & 69.20 & 17.30
\end{array}
$$

f) Specific gravity.-1.434

g) Saccharifying quality. $-27.45 \%$

h) Hardness. (kilo)

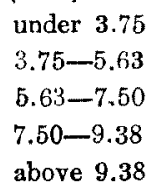




$\begin{array}{ccc}\text { average. } & \max & \min . \\ 5.57 & 8.93 & 3.00\end{array}$

i) Volume of $100 \mathrm{~g}$. $124.0 \mathrm{c.c}$.

j) Weight of 1,000 grains. $-19.3 \mathrm{~g}$.

n) Absorption of water.

$\begin{array}{ccc}\text { time steeped. } & \text { water absorbed. } & \% \text { absorbed. } \\ 30 \mathrm{~m} . & 7.92 \mathrm{~g} . & 15.84 \\ 2 \mathrm{~h} . & 10.80 " & 21.60\end{array}$

p) Chemical composition :-

$\begin{array}{lrr}\text { Water } & 12.96 & - \\ \text { D. R. sugar } & 0.90 & 1.03 \\ \text { Dextrin } & 1.27 & 1.46 \\ \text { Starch } & 77.34 & 88.85 \\ \text { Crude fat } & 0.89 & 1.02 \\ \quad \text { " fibre } & 0.80 & 0.92 \\ \quad \text { " protein } & 6.62 & 7.48 \\ \text { Ash } & 0.65 & 0.74 \\ \text { Phosphoric acid } & 0.24 & 0.27\end{array}$

IV) Production of Kagawa prefecture.

a) Percentage of full grown grains.-94.6\%

b) External appearance and luster.-Nearly same as before.

c) Size of grains. (m.m.)

\begin{tabular}{|c|c|c|c|c|c|}
\hline \multicolumn{2}{|c|}{ thickness above } & 2.2 & $10.80 \mathrm{~g}$ & \multicolumn{2}{|c|}{5 grains. } \\
\hline "I & $\prime \prime$ & 2.0 & $45.70 . " 1$ & $2 \cdot 3$ & $" \prime$ \\
\hline " & $" 1$ & 1.8 & $39.00 " 1$ & 20 & 11 \\
\hline " & under & $\begin{array}{c}1.8 \\
\text { average. }\end{array}$ & $\begin{array}{l}4.20 " \\
\max .\end{array}$ & $\begin{array}{c}2 \\
\text { min. }\end{array}$ & " \\
\hline & ngth & 4.95 & 5.15 & 4.60 & \\
\hline & idth & 2.83 & 2.95 & 2.65 & \\
\hline
\end{tabular}

d) Percentage of Shinziromai.-4.8\%

e) Depth of longitudinal furrow. $(\mu)$

$\begin{array}{rrr}\text { average. } & \max . & \min . \\ 35.98 & 69.20 & 17.30\end{array}$

f) Specific gravity. -1.429

g) Saccharifying quality.-32.71\%

h) Hardness. (kilo)

\begin{tabular}{|c|c|c|}
\hline under 3.75 & & 28 grains \\
\hline $3.75-5.63$ & & $19 \prime \prime$ \\
\hline $5.63-7.50$ & & " \\
\hline $7.50-9.38$ & & - \\
\hline above 9.38 & & - \\
\hline verage. & $\max$. & $\min$. \\
\hline 3.90 & 6.56 & 2.63 \\
\hline
\end{tabular}

i) Volume of $100 \mathrm{~g} . \longrightarrow 123.5 \mathrm{c} . \mathrm{c}$. 
j) Weight of 1,000 grains. $-19.3 \mathrm{~g}$.

n) Absorption of water.

$\begin{array}{ccc}\text { time steeped. } & \text { water absorbed. } & \% \text { absorbed. } \\ 30 \mathrm{~m} & 7.65 \mathrm{~g} . & 15.30 \\ 2 \mathrm{~h} & 10.47 \mathrm{\prime \prime} & 20.94\end{array}$

p) Chemical composition :-

$\begin{array}{lrc}\text { Water } & & \text { unhydrous } \\ \text { D. R. sugar } & 13.62 & - \\ \text { Dextrin } & 0.64 & 0.75 \\ \text { Starch } & 0.67 & 0.78 \\ \text { Crude fat } & 77.79 & 90.06 \\ \quad \text { " fibre } & 0.88 & 1.01 \\ \quad \text { " protein } & 0.27 & 0.31 \\ \text { Ash } & 6.70 & 8.00 \\ \text { Phosphoric acid } & 0.61 & 0.71 \\ \end{array}$

V) Production of Alita prefecture.

a) Percentage of full grown grains.- $96.19 \%$

b) External appearance and luster.- Somewhat brown straw colour, grains were fat and middle size.

c) Size of grains. (m.m.)

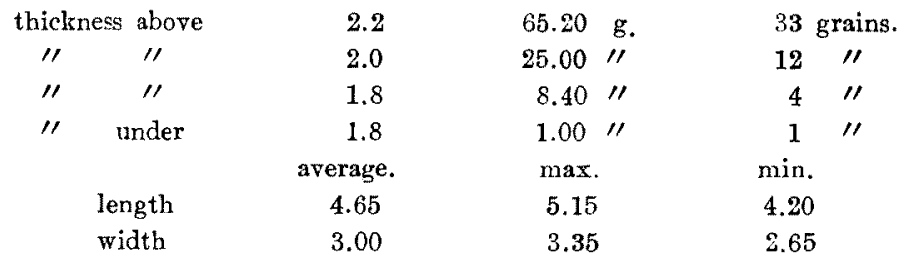

d) Percentage of Shinziromai._- $30.8 \%$

e) Depth of longitudinal furrow. $(\mu)$

$\begin{array}{ccc}\text { average. } & \max . & \min . \\ 50.34 & 86.50 & 17.30\end{array}$

f) Specific gravity. -1.429

g) Saccharifying quality.—37.11\%

h) Hardness. (kilo)

$\begin{array}{llcl}\text { under } 3.75 & & 25 & \text { grains. } \\ 3.75-5.63 & & 21 & \prime \prime \\ 5.63-7.50 & & 4 & \prime \prime \\ 7.50-9.38 & & - & \\ \text { above } 9.38 & & - & \\ \text { average. } & \max . & & \min . \\ 3.84 & 6.15 & & 2.25\end{array}$

i) Volume of $100 \mathrm{~g} .-123.5 \mathrm{c} . \mathrm{c}$.

j) Weight of 1,000 grains. $-22.0 \mathrm{~g}$.

n) Absorption of water. 


$\begin{array}{ccc}\text { time steeped. } & \text { water absorbed. } & \% \text { absorbed. } \\ 30 \mathrm{~m} . & 5.40 & 10.80 \\ 2 \mathrm{~h} . & 8.50 & 17.00\end{array}$

p) Chemical composition :-

$\begin{array}{lrc} & & \text { unhydrous. } \\ \text { Water } & 14.21 & - \\ \text { D. R. sugar } & 1.24 & 1.45 \\ \text { Dextrin } & 1.29 & 1.50 \\ \text { Starch } & 76.34 & 88.98 \\ \text { Crude fat } & 0.25 & 0.30 \\ \quad \text { " fibre } & 0.26 & 0.30 \\ \quad \text { " protein } & 7.00 & 8.16 \\ \text { Ash } & 0.49 & 0.57 \\ \text { Phosphoric acid } & 0.15 & 0.17\end{array}$

VI) Production of Yamagata prefecture.

a) Percentage of full grown grains._- $88.0 \%$

b) External appearance and luster.-Greyish white colour, grains were not fat and middle size.

c) Size of grains. (m.m.)

\begin{tabular}{|c|c|c|c|c|c|}
\hline thicl & above & 2.2 & 30.80 & \multicolumn{2}{|c|}{16 grains. } \\
\hline$\prime \prime$ & $\prime \prime$ & 2.0 & 40.60 & 20 & 11 \\
\hline$\prime \prime$ & $\prime \prime$ & 1.8 & 23.90 & 12 & 11 \\
\hline " & under & $\begin{array}{r}1.8 \\
\text { average. }\end{array}$ & $\begin{array}{l}4.00 \\
\max .\end{array}$ & $\begin{array}{r}2 \\
\min .\end{array}$ & 11 \\
\hline & gth & 4.93 & 5.40 & 4.45 & \\
\hline & $d t h$ & 2.87 & 3.10 & 2.45 & \\
\hline
\end{tabular}

d) Percentage of Shinziromai._- $13.6 \%$

e) Depth of longitudinal furrow. $(\mu)$

$\begin{array}{rcr}\text { average. } & \max . & \min . \\ 39.01 & 69.20 & 17.30\end{array}$

f) Specific gravity. 1.434

g) Saccharifying quality.-29.12\%

h) Hardness. (kilo)

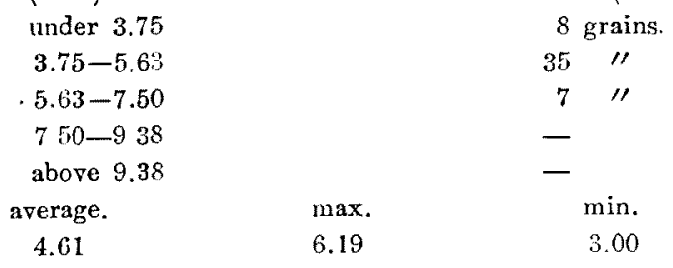

i) Volume of $100 \mathrm{~g} . \longrightarrow 126.0$ c.c.

j) Weight of 1,000 grains.-20.55g.

n) Absorption of water.

$\begin{array}{ccc}\text { time steeped. } & \text { water absorbed. } & \% \text { absorbed. } \\ 30 \mathrm{~m} . & 7.62 \mathrm{~g} . & 15.24 \\ 2 \mathrm{~h} . & 8.88 \mathrm{\prime l} & 17.76\end{array}$


[Vol. 3,

\begin{tabular}{|c|c|c|c|}
\hline & $\mathrm{P}_{2} \mathrm{O}_{6}$ & 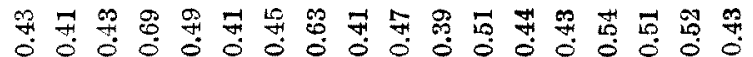 & 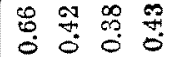 \\
\hline & Ash & 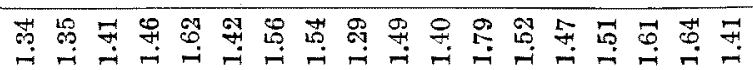 & 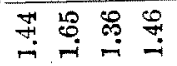 \\
\hline & Crude protein & 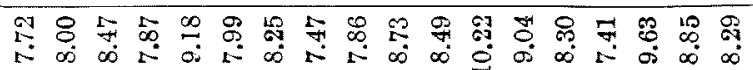 & 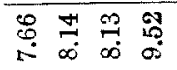 \\
\hline & Crude fibre & 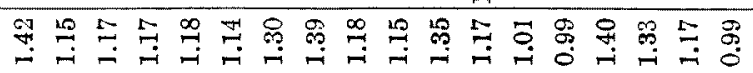 & 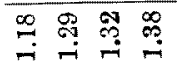 \\
\hline & Crude fat & 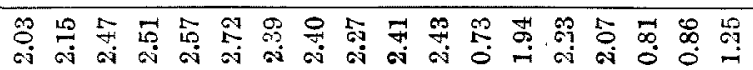 & 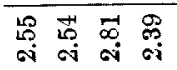 \\
\hline & Starch & 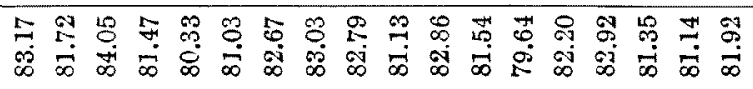 & $\begin{array}{l}\therefore \\
\therefore\end{array}$ \\
\hline & Dextrin & 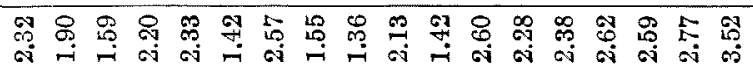 & 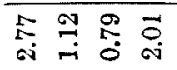 \\
\hline & D. R. sugar & 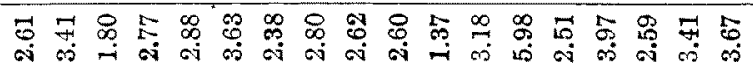 & 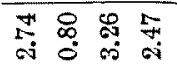 \\
\hline & Water & 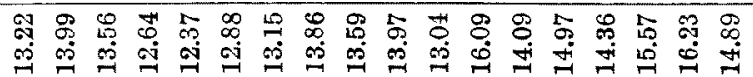 & 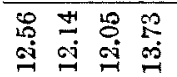 \\
\hline & $\begin{array}{l}\text { Depth of } \\
\text { embryo cavity }\end{array}$ & 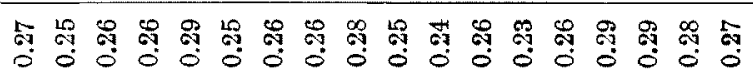 & 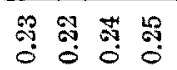 \\
\hline 焉 & $\begin{array}{l}\text { Thickness of } \\
\text { bran layer. }(\mu)\end{array}$ & 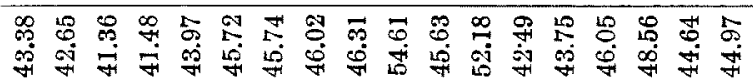 & 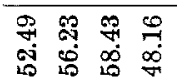 \\
\hline & $\begin{array}{l}\text { Weight of } \\
\text { 1,000grain. (g.) }\end{array}$ & 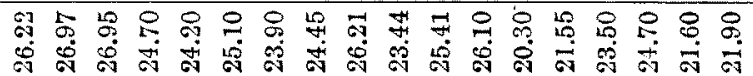 & 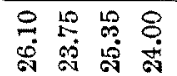 \\
\hline & $\begin{array}{l}\text { Volume of } \\
\text { loog. (c.c.) }\end{array}$ & 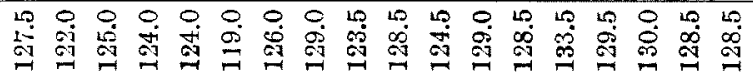 & 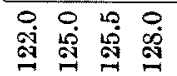 \\
\hline $\begin{array}{l}4 \\
4\end{array}$ & $\begin{array}{l}\text { Absorption of } \\
\text { water. (\%) }\end{array}$ & 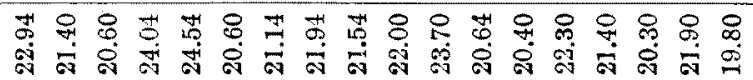 & 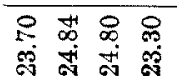 \\
\hline & Hardness. (kilo) & 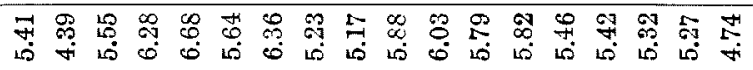 & 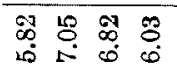 \\
\hline & $\begin{array}{l}\text { Saccharifying } \\
\text { quality. }(\%)\end{array}$ & 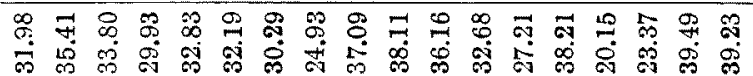 & 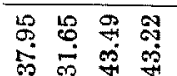 \\
\hline & Specific gravity. & 贲 & 菒 \\
\hline & $\begin{array}{l}\text { Depth of longi- } \\
\text { tudinal furrow. }(\mu .)\end{array}$ & 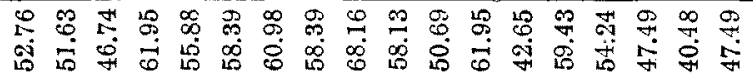 & 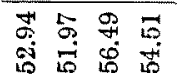 \\
\hline & Shinziromai & 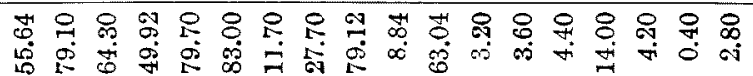 & 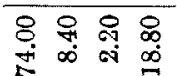 \\
\hline & $\begin{array}{l}\text { External } \\
\text { appearance and } \\
\text { luster. }\end{array}$ & 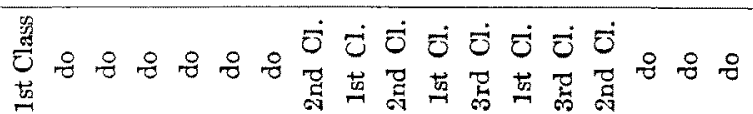 & 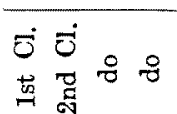 \\
\hline & $\begin{array}{l}\% \text { of full } \\
\text { grown grain. }\end{array}$ & 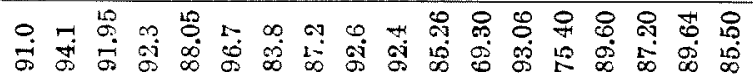 & 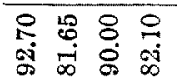 \\
\hline & & $\varangle \oplus$ & \\
\hline
\end{tabular}




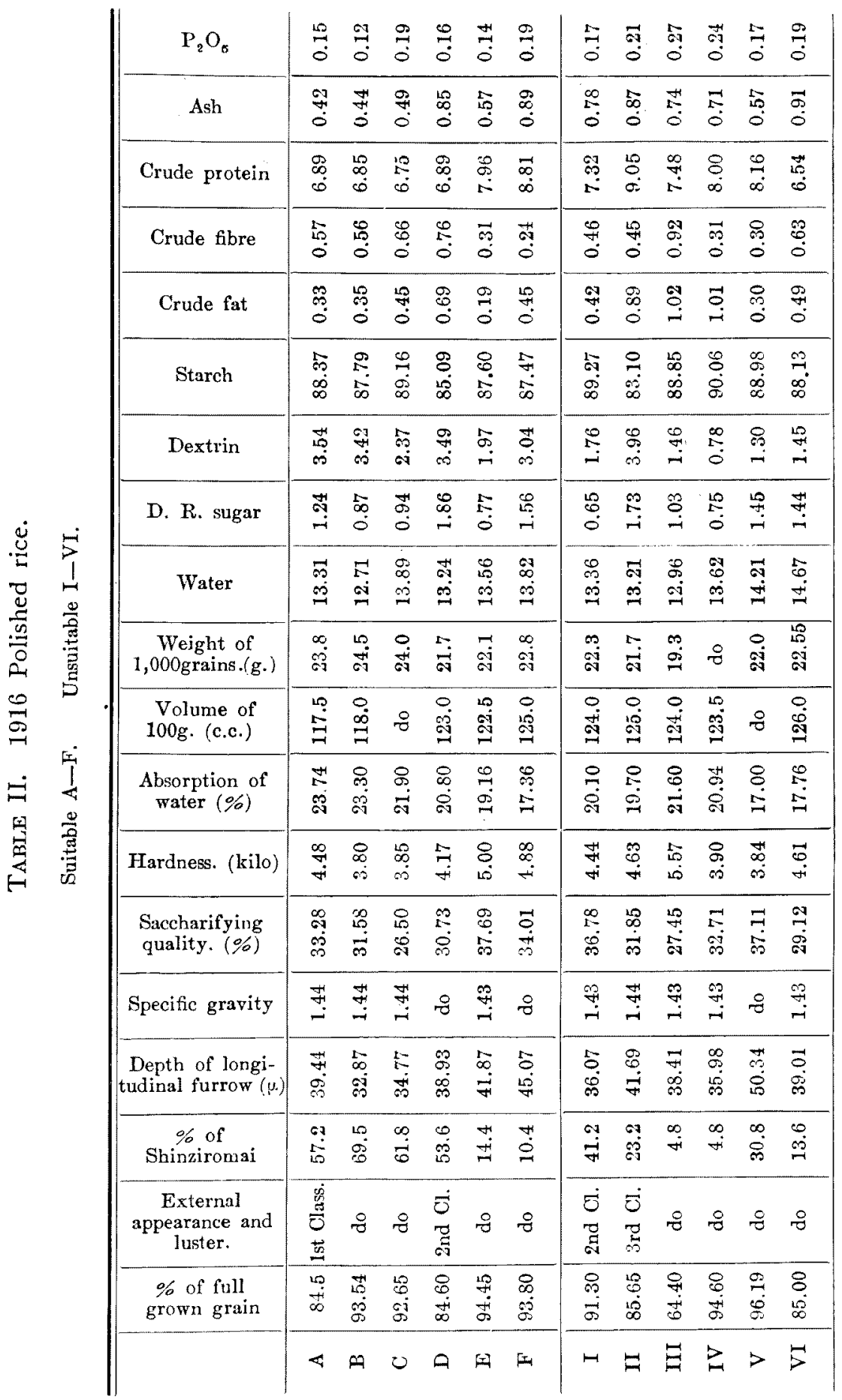


p) Chemical composition :-

$\begin{array}{lrc} & & \text { unhydrous. } \\ \text { Water } & 14.67 & - \\ \text { D. R. sugar } & 1.23 & 1.44 \\ \text { Dextrin } & 1.24 & 1.45 \\ \text { Starch } & 75.20 & 88.13 \\ \text { Crude fat } & 0.42 & 0.49 \\ \quad \text { " fibre } & 0.54 & 0.63 \\ \quad \text { " protein } & 6.34 & 6.54 \\ \text { Ash } & 0.78 & 0.91 \\ \text { Phosphoric acid } & 0.16 & 0.19\end{array}$

\section{Summary of the 1916 production.}

Percentage of full grown grains and Shinziromai, weight of 1,000 grains, and the sum of starch, dextrin and sugar, are found to be greater in the suitable materials for the Saké brewing, whilst volume of $100 \mathrm{~g}$, thickness of bran layer, and the conetnts of crude fat, crude protein, crude fibre, ash and phosphoric acid, are found to be smaller. Specific gravity, saccharifying quality, hardness, water and absorption of water were found to be moderate. The external appearance and luster should be fine. Seven varieties (L-R) produced in the districts rather unfitted for rice plantation were omitted from the judgment since these were locally regarded as the suitable materials for the Sake brewing, but not in the general opinion.

\section{Production.}

a) Husked rice, suitable for the Sake brewing. 20 (A-T) varieties.

A) Production of Okayama prefecture.

a) Percentage of full grown grains.-90.33\%

b) External appearance and luster.-Light straw yellow waxy luster, grains were fat and large, impurities were rare.

c) Size of grains. (m.m.)

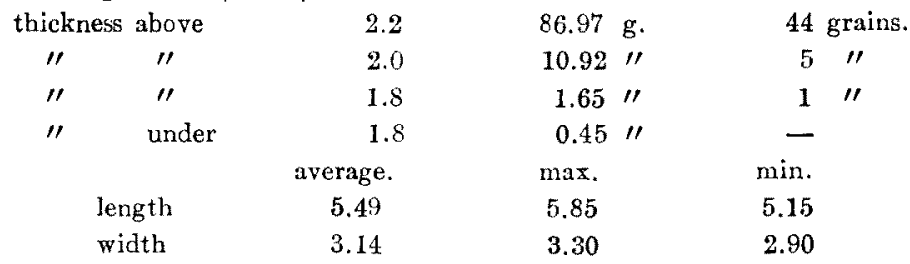

d) Percentage of Shinziromai.- $95.24 \%$

e) Depth of longitudinal furrow. $(\mu)$
average.
max.
min.
41.52
69.20
17.30

f) Specific gravity. -1.399 
g) Saccharifying quality.-27.30\%

h) Hardness. (kilo)

\begin{tabular}{|c|c|c|c|}
\hline under 3.75 & & & grains \\
\hline $3.75-5.63$ & & 36 & $\prime \prime$ \\
\hline $5.63-7.50$ & & 6 & $"$ \\
\hline $7.50-9.38$ & & - & \\
\hline above 9.38 & & - & \\
\hline 3 verage. & $\max$. & & min. \\
\hline 4.54 & 6.63 & & 2.53 \\
\hline
\end{tabular}

i) Volume of $100 \mathrm{~g}$. - 124.0c.c.

j) Weight of 1,000 grains. $-28.23 \mathrm{~g}$.

k) Volume of 1,000 grains. -20.25 c.c.

m) Thickness of bran layer. ( $(x)$

$\begin{array}{ccc}\text { average. } & \max . & \min . \\ 50.27 & 59.40 & 41.63\end{array}$

n) Absorption of water.

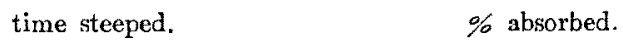

o) Depth of embryo cavity :-

$\begin{array}{ccc}\text { average. } & \max . & \text { min. } \\ 0.24 & 0.29 & 0.21\end{array}$

p) Chemical composition :--

$\begin{array}{lrr}\text { Water } & 12.88 & - \\ \text { D. R. sugar } & 0.96 & 1.11 \\ \text { Starch } & 73.16 & 84.42 \\ \text { Crude fat } & 2.31 & 2.55 \\ \quad \text { " fibre } & 1.65 & 1.90 \\ \quad \text { " protein } & 7.96 & 9.19 \\ \text { Ash } & 1.19 & 1.37 \\ \text { Phosphoric acid } & 0.44 & 0.51\end{array}$

B) Production of Okayama prefecture.

a) Percentage of full grown grains. $-90.48 \%$

b) External appearance and luster.-Nearly same as A).

c) Size of grains. (m.m.)

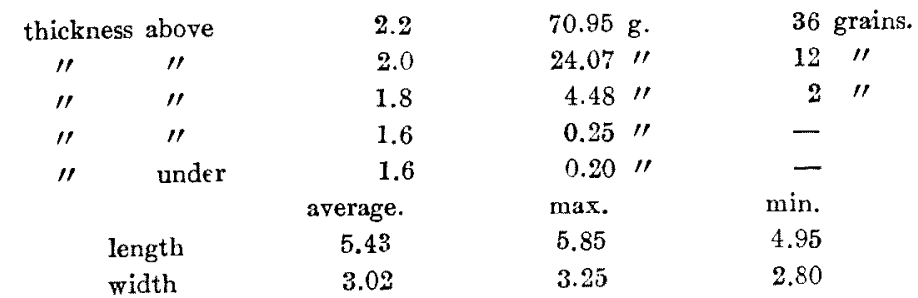

d) Percentage of Shinziromai.- $58.0 \%$ 
e) Depth of longitudinal furrow. $(\mu)$

\begin{tabular}{|c|c|c|c|}
\hline $\begin{array}{c}\text { average. } \\
31.57\end{array}$ & $\begin{array}{l}\max . \\
51.90\end{array}$ & & in. \\
\hline \multicolumn{4}{|c|}{ ravity. 1.399} \\
\hline \multicolumn{4}{|c|}{$\begin{array}{l}\text { ying quality. } \longrightarrow 30.04 \% \\
\text { (kilo) }\end{array}$} \\
\hline under 3.75 & & & grains. \\
\hline $3.75-5.63$ & & 28 & $" \prime$ \\
\hline $5.63-7.50$ & & 20 & $" \prime$ \\
\hline $7.50-9.38$ & & - & \\
\hline above 9.33 & & - & \\
\hline average. & $\max$. & & nin. \\
\hline 5.32 & 7.28 & & 3.19 \\
\hline
\end{tabular}

i) Volume of $100 \mathrm{~g}$. $122.0 \mathrm{c} . \mathrm{c}$.

j) Weight of 1,000 grains.—-26.45g.

k) Volume of 1,000 grains.-18.9c.c.

1) Phytin.- $0.19 \%$

m) Thickness of bran layer. $(\mu)$

$$
\begin{array}{ccc}
\text { average. } & \text { max. } & \min . \\
45.60 & 49.28 & 40.50
\end{array}
$$

n) Absorption of water.

time steeped. $\%$ absorbed.
$24 \mathrm{~h}$.
23.22

o) Depth of embryo cavity :-

$\begin{array}{ccc}\text { arerage. } & \max . & \min . \\ 0.25 & 0.31 & 0.19\end{array}$

p) Chemical composition :-

$\begin{array}{lrr}\text { Water } & 13.07 & - \\ \text { D. R. sugar } & 0.92 & 1.08 \\ \text { Starch } & 73.10 & 85.23 \\ \text { Crude fat } & 1.91 & 2.23 \\ \quad \text { " fibre } & 1.49 & 1.73 \\ \quad \text { " protein } & 7.11 & 8.28 \\ \text { Ash } & 1.23 & 1.44 \\ \text { Phosphoric acid } & 0.50 & 0.58\end{array}$

C) Production of Niigata prefecture.

a) Percentage of full grown grains.-95.0\%

b) External appearance and luster.-Brown straw yellow colour, destitute of luster, grains were not fat.

c) Size of grains. (m.m.)

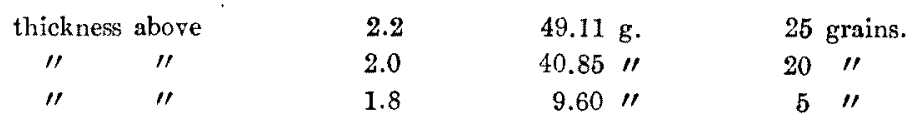




$\begin{array}{ccccc}\prime \prime & 1.6 & 0.27 \prime \prime & - \\ \prime \prime & 1.6 & 0.06 \prime \prime & - \\ & \text { under } & \text { average. } & \text { max. } & \text { min. } \\ & 5.27 & 5.80 & 4.90 \\ \text { length } & 2.97 & 3.15 & 2.60\end{array}$

d) Percentage of Shinziromai.-12.5\%

e) Depth of longitudinal furrow. $(\mu)$ average. $\max$. $41.74 \quad 69.20$

f) Specific gravity.-1.399

g) Saccharifying quality.-30.15\%

h) Hardness. (kilo)

\begin{tabular}{|c|c|c|}
\hline under 3.75 & & 19 grains. \\
\hline $3.75-5.63$ & & $31 " \prime$ \\
\hline $5.63-7.50$ & & - \\
\hline $7.50-9.38$ & & 一 \\
\hline above 9.38 & & - \\
\hline $\begin{array}{c}\text { average. } \\
4.10\end{array}$ & $\max$ & $\begin{array}{r}\min \\
2.81\end{array}$ \\
\hline
\end{tabular}

i) Volume of $100 \mathrm{~g} . \longrightarrow 131.0 \mathrm{c} . \mathrm{c}$.

j) Weight of 1,000 grains. $-24.22 \mathrm{~g}$.

k) Volume of 1,000 grains._-16.6c.c.

m) Thickness of bran layer. $(\mu)$

$\begin{array}{ccc}\text { average. } & \max . & \min , \\ 45.77 & 53.10 & 37.80\end{array}$

n) Absorption of water.

time steeped. $\quad \%$ absorbed.

$24 \mathrm{~h}$.

19.1

o) Depth of embryo cavity :-

$\begin{array}{lll}0.23 & 0.27 & 0.19\end{array}$

min.

p) Chemical composition :-

$\begin{array}{lrr}\text { Water } & 14.24 & - \\ \text { D. R. sugar } & 1.11 & 1.30 \\ \text { Starch } & 72.22 & 84.35 \\ \text { Crude fat } & 2.26 & 2.64 \\ \quad \text { " fibre } & 0.99 & 1.15 \\ \quad \text { " protein } & 7.88 & 9.20 \\ \text { Ash } & 1.18 & 1.38 \\ \text { Phosphoric acid } & 0.44 & 0.51\end{array}$

D) Production of Hyogo prefecture.

a) Percentage of full grown grains.- $89.86 \%$

b) External appearance and luster. Straw yellow waxy luster, grains were large and fat. 
c) Size of grains. (m.m.)

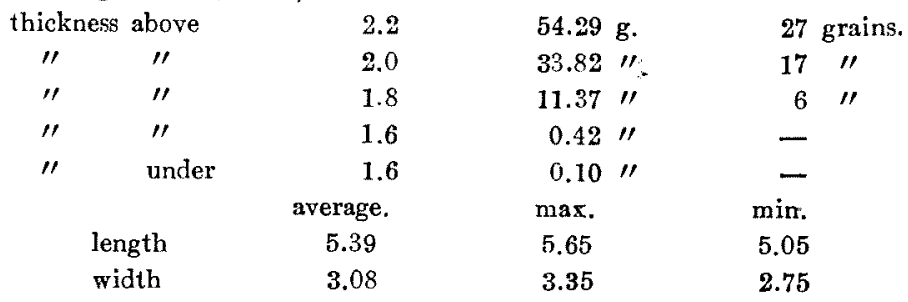

d) Percentage of Shinziromai. $-77.0 \%$

e) Depth of longitudinal furrow. $(\mu)$

$\begin{array}{ccc}\text { average. } & \max . & \text { min. } \\ 67.34 & 103.80 & 34.60\end{array}$

f) Specific gravity.-1.409

1.42 (Shinziromai)

g) Saccharifying quality._- 30.28

h) Hardness. (kilo)

\begin{tabular}{|c|c|c|c|}
\hline under 3.75 & & & grains. \\
\hline $3.75-5.63$ & & 36 & $" \prime$ \\
\hline $5.63-7.50$ & & 8 & " \\
\hline $7.50-9.38$ & & - & \\
\hline above 9.38 & & - & \\
\hline $\begin{array}{c}\text { average. } \\
4.69\end{array}$ & $\begin{array}{l}\max . \\
6.56\end{array}$ & & \\
\hline
\end{tabular}

i) Volume of $100 \mathrm{~g} .-122.0$ c.c.

j) Weight of 1,000 grains. $-25.78 \mathrm{~g}$.

$28.00 \mathrm{~g}$. (Shinziromai)

k) Volume of 1,000 grains._18.4c.c.

20.3c.c. (Shinziromai)

m) Thickness of bran layer. ( $(x)$

$\begin{array}{rlc}\text { average. } & \max . & \min . \\ \mathbf{4 4 . 6 2} & 49.28 & 40.28\end{array}$

n) Absorption of water.

time steeped. $\%$ absorbed.

$24 \mathrm{~h} . \quad 21.96$

o) Depth of embryo cavity :-

$\begin{array}{ccc}\text { average. } & \max . & \min . \\ 0.28 & 0.31 & 0.24\end{array}$

p) Chemical composition :-

Water

D. R. sugar

Stareh

Crude fat
13.14

0.68

74.41

2.10 unhydrous.

$$
\begin{array}{r}
0.79 \\
85.72 \\
2.41
\end{array}
$$




$\begin{array}{rll}\prime \prime \text { fibre } & 1.15 & 1.32 \\ \prime \prime \text { protein } & 7.29 & 8.40 \\ \text { Ash } & 1.18 & 1.36 \\ \text { Phosphoric acid } & 0.20 & 0.23\end{array}$

E) Production of Hyogo prefecture.

a) Percentage of full grown grains. $-88.88 \%$

b) External appearance and luster.-Nearly same as before.

c) Size of grains. (m.m.)

\begin{tabular}{|c|c|c|c|c|c|c|}
\hline thick & above & 2.2 & 55.72 & & 28 & grains. \\
\hline " & $\prime \prime$ & 2.0 & 33.59 & $\prime \prime$ & 17 & $\prime \prime$ \\
\hline "' & "I & 1.8 & 9.68 & $" \prime$ & 5 & "' \\
\hline$\prime \prime$ & $\prime \prime$ & 1.6 & 0.63 & $" \prime$ & - & \\
\hline " & under & 1.6 & 0.05 & $" 1$ & - & \\
\hline & & average. & $\max$ & & $\min$. & \\
\hline & igth & 5.23 & 5.65 & & 5.00 & \\
\hline & dth & 3.13 & 3.33 & & 3.00 & \\
\hline
\end{tabular}

d) Percentage of Shinziromai._- $69.64 \%$

e) Depth of longitudinal furrow. $(\mu)$

$\begin{array}{ccc}\text { average. } & \max . & \min . \\ 57.18 & 86.50 & 25.95\end{array}$

f) Specific gravity. - 1.409

g) Saccharifying quality. $-32.06 \%$

h) Hardness. (kilo)

\begin{tabular}{|c|c|c|}
\hline under 3.75 & & 12 grains. \\
\hline $3.75-5.63$ & & $31 \prime \prime$ \\
\hline $5.63-7.50$ & & $\prime \prime$ \\
\hline $7.50-9.38$ & & - \\
\hline above 9.38 & & - \\
\hline average. & $\max$. & $\min$. \\
\hline 4.48 & 6.56 & 2.78 \\
\hline
\end{tabular}

i) Volume of $100 \mathrm{~g} .-126.5 \mathrm{c} . \mathrm{c}$.

j) Weight of 1,000 grains. $-25.35 \mathrm{~g}$.

k) Volume of 1,000 grains.—18.1c.c.

m) Thickness of bran layer. $(\mu)$ $\begin{array}{ccr}\text { average. } & \max . & \min . \\ 44.54 & 52.88 & 36.45\end{array}$

n) Absorption of water. time steeped. $24 \mathrm{~h}$.

$\%$ absorbed. 21.6

o) Depth of embryo cavity :$\begin{array}{cc}\text { average. } & \max . \\ 0.28 & 0.32\end{array}$ min. 0.22

p) Chemical composition :- 


$\begin{array}{lrc} & & \text { unhydrous. } \\ \text { Water } & 13.59 & - \\ \text { D. R. sugar } & 1.20 & 1.40 \\ \text { Starch } & 72.56 & 84.30 \\ \text { Crude fat } & 2.10 & 2.44 \\ \quad \text { "fibre } & 1.09 & 1.27 \\ \quad \text { " protein } & 7.96 & 9.25 \\ \text { Ash } & 1.16 & 1.35 \\ \text { Phosphoric acid } & 0.42 & 0.48\end{array}$

F) Production of Hyogo prefecture.

a) Percentage of full grown grains.- $93.4 \%$

b) External appearance and luster.-Nearly same as before.

c) Size of grains. (m.m.)

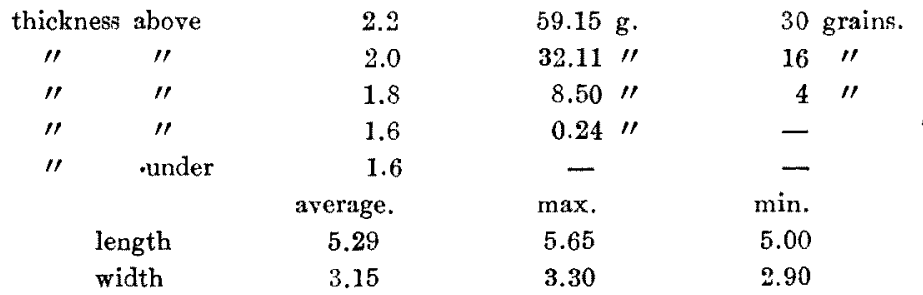

d) Percentage of Shinziromai.-68.0\%

e) Depth of longitudinal furrow. $(\mu)$

$\begin{array}{rcr}\text { average. } & \text { max. } & \text { min. } \\ 58.60 & 103.80 & 34.60\end{array}$

f) Specific gravity.-1.409

g) Saccharifying quality. $-26.32 \%$

h) Hardness. (kilo)

\begin{tabular}{|c|c|c|}
\hline under 3.75 & & 17 grains. \\
\hline $3.75-5.63$ & & $30 \prime \prime$ \\
\hline $5.63-7.50$ & & $\prime \prime$ \\
\hline $7.50-9.38$ & & 一 \\
\hline above 9.38 & & 一 \\
\hline average. & $\max$. & min. \\
\hline 4.24 & 5.68 & 2.33 \\
\hline
\end{tabular}

i) Volume of $100 \mathrm{~g}$ - 123.0c.c.

j) Weight of 1,000 grains.—25.12g.

k) Volume of 1,000 grains.—18.00c.c.

m) Thickness of bran layer. $(\mu)$

$\begin{array}{ccc}\text { average. } & \max . & \min . \\ 43.28 & 50.85 & 36.90\end{array}$

n) Absorption of water.

time steeped. $24 \mathrm{~h}$. 
o) Depth of embryo cavity :-

$$
\begin{array}{clc}
\text { average. } & \max . & \min . \\
0.28 & 0.33 & 0.24
\end{array}
$$

p) Chemical composition :-

$\begin{array}{lrr}\text { Water } & 13.31 & - \\ \text { D. R. sugar } & 1.14 & 1.31 \\ \text { Starch } & 73.64 & 84.79 \\ \text { Crude fat } & 2.11 & 2.42 \\ \quad \text { " fibre } & 1.01 & 1.16 \\ \quad \text { " protein } & 7.96 & 9.17 \\ \text { Ash } & 1.00 & 1.03 \\ \text { Phosphoric acid } & 0.52 & 0.60\end{array}$

G) Production of Fuknoka prefecture.

a) Pecentage of full grown grains.- $-86.38 \%$

b) External appearance and luster.—Brownish straw colour, destitute of luster, grains were not fat and of middle size, impurities were tolerably abundant.

c) Size of grains. (m.m.)

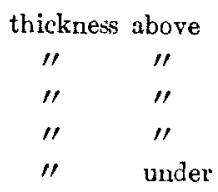

length width

2.2
2.0
1.8
1.6
1.6
arerage.
5.15

3.00

$21.51 \mathrm{~g}$.
$40.38 "$
$31.88 "$
$4.39 "$
$1.84 "$
$\max$.
5.50

3.25

$\begin{aligned} 11 & \text { grains. } \\ 20 & \prime \prime \\ 16 & \prime \prime \\ 2 & \prime \prime \\ 1 & \prime \prime \\ \min . & \\ 4.60 & \\ 2.65 & \end{aligned}$

d) Percentage of Shinziromai.- $-20.6 \%$

e) Depth of longitudinal furrow. $(\mu)$

$$
\begin{array}{crr}
\text { average. } & \max . & \min . \\
58.39 & 78.75 & 17.30
\end{array}
$$

\begin{tabular}{|c|c|c|}
\hline under 3.75 & & \\
\hline $3.75-5.63$ & & 15 \\
\hline $5.63-7.50$ & & 28 \\
\hline $7.50-9.38$ & & 2 \\
\hline above 9.38 & & - \\
\hline verage. & $\max$. & \\
\hline
\end{tabular}

f) Specific gravity.-1.399

g) Saccharifying quality.-31.26\%.

h) Hardness. (kilo)

i) Volume of $100 \mathrm{~g} \cdot-129.0 \mathrm{c} . \mathrm{c}$.

j) Weight of 1,000 grains. $-23.05 \mathrm{~g}$.

k) Volume of 1,000 grains.-16.55c.c. 
1) Phytin- $-0.56 \%$

m) Thickness of bran layer. $(\mu)$

$\begin{array}{ccc}\text { average. } & \max . & \min . \\ 44.06 & 48.38 & 40.73\end{array}$

n) Absorption of water.

time steeped. $\%$ absorbed.
$24 \mathrm{~h}$.
24.46

o) Depth of embryo cavity :-

$\begin{array}{ccc}\text { average. } & \max . & \min . \\ 0.26 & 0.32 & 0.18\end{array}$

p) Chemical composition :-

$\begin{array}{lrc} & & \text { unhydrous. } \\ \text { Water } & 12.46 & - \\ \text { D. R. sugar } & 1.00 & 1.15 \\ \text { Starch } & 73.47 & 84.18 \\ \text { Crude fat } & 2.44 & 2.80 \\ \quad \text { "fibre } & 1.39 & 1.58 \\ \quad \prime \prime \text { protein } & 7.71 & 8.83 \\ \text { Ash } & 1.27 & 1.46 \\ \text { Phosphoric acid } & 0.54 & 0.69\end{array}$

H) Production of Kumamoto prefecture.

a) Percentage of full grown grains. $-89.23 \%$

b) External appearance and luster.-Nearly same as A).

c) Size of grains. (m.m.)

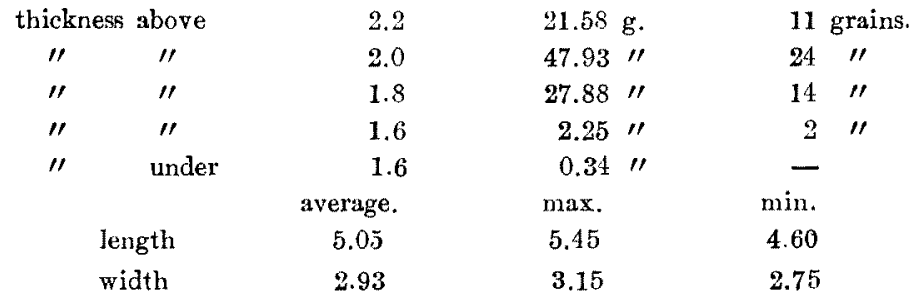

d) Percentage of Shinziromai._- $32.4 \%$

e) Depth of longitudinal furrow. $(\mu)$

$\begin{array}{rlr}\text { average. } & \max . & \min . \\ 55.14 & 86.50 & 17.30\end{array}$

f) Specific gravity.- 1.404

g) Saccharifying quality.-34.18\%

h) Hardness. (kilo)

\begin{tabular}{|c|c|c|}
\hline under 3.75 & & grains. \\
\hline $3.75-5.63$ & 25 & $\prime \prime$ \\
\hline $3.63-7.50$ & 21 & $" \prime$ \\
\hline $7.50-9.38$ & 1 & $" \prime$ \\
\hline above 9.38 & - & \\
\hline
\end{tabular}




$$
\begin{array}{ccc}
\text { average. } & \text { max. } & \text { min. } \\
5.35 & 7.97 & \mathbf{2 . 6 3}
\end{array}
$$

i) Volume of $100 \mathrm{~g}$ - -121.0 c.c.

j) Weight of 1,000 grains.-22.37g.

k) Volume of 1,000 grains._-16.0c.c.

1) Phytin. 17.1c.c. (Shinziromai)

m) Thickness of bran layer. $(\mu)$

$$
\begin{array}{ccc}
\text { average. } & \max . & \min . \\
4 \mathbf{2 . 4} & 47.03 & 38.48
\end{array}
$$

n) Absorption of water. time steeped. $\%$ absorbed. $24 \mathrm{~h}$. 23.48

o) Depth of embryo cavity :-

$$
\begin{array}{ccc}
\text { average. } & \max . & \min . \\
0.28 & 0.34 & 0.25
\end{array}
$$

p) Chemical composition :-

\begin{tabular}{|c|c|c|c|c|c|c|}
\hline thick & above & 2.2 & 40.42 & & 20 & grains. \\
\hline$\prime \prime$ & $\prime \prime$ & 2.0 & 44.21 & $" \prime$ & 29 & $\prime \prime$ \\
\hline$" \prime$ & "I & 1.8 & 14.19 & "I & 7 & " \\
\hline$\prime \prime$ & " & 1.6 & 0.94 & $\prime \prime$ & 1 & $\prime \prime$ \\
\hline " & under & 1.6 & 0.18 & $\prime \prime$ & - & \\
\hline & & ave rage. & $\max$. & & min. & \\
\hline & igth & 5.19 & 5.55 & & 5.00 & \\
\hline & dth & 3.00 & 3.25 & & 2.75 & \\
\hline
\end{tabular}

$\begin{array}{lrc} & & \text { unhydrous. } \\ \text { Water } & 12.53 & - \\ \text { D. R. sugar } & 1.08 & 1.23 \\ \text { Starch } & 73.34 & 83.91 \\ \text { Crude fat } & 2.50 & 2.86 \\ \quad \text { " fibre } & 1.24 & 1.41 \\ \quad \text { " protein } & 8.05 & 9.21 \\ \text { Ash } & 1.21 & 1.38 \\ \text { Phosphoric acid } & 0.50 & 0.57\end{array}$

I) Production of Saga prefecture.

a) Percentage of full grown grains.- $89.63 \%$

b) External appearance and luster.—-Nearly same as before.

c) Size of grains. (m.m.)

d) Percentage of Shinziromai.- $31.5 \%$

e) Depth of longitudinal furrow. $(\mu)$

$\begin{array}{rrr}\text { average } & \text { max. } & \text { min. } \\ 61.63 & 95.15 & 34.60\end{array}$

f) Specific gravity. -1.409

g) Saccharifying quality. $-32.07 \%$ 
h) Hardness. (kilo)

$$
\begin{gathered}
\text { under } 3.75 \\
3.75-5.63 \\
5.63-7.50 \\
7.50-9.38 \\
\text { above } 9.38 \\
\text { average. } \\
5.60
\end{gathered}
$$

$\max$.

$\begin{array}{lc} & 3 \text { grains. } \\ & 21 " \prime \\ & 25 \quad " \\ & 1 \quad \prime \\ & - \\ \max . & \min . \\ 7.54 & 3.38\end{array}$

i) Volume of $100 \mathrm{~g}$. $125.0 \mathrm{c} . \mathrm{c}$.

j) Weight of 1,000 grains. $-23.7 \mathrm{~g}$.

k) Volume of 1,000 grains. - 16.9c.c.

m) Thickness of bran layer. $(\mu)$

$$
\begin{array}{ccc}
\text { average. } & \text { max. } & \text { min. } \\
48.89 & 50.85 & 36.00
\end{array}
$$

n) Absorption of water.

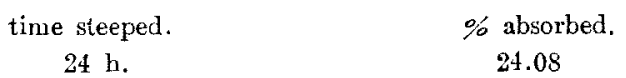

o) Depth of embryo cavity :-

$\begin{array}{clc}\text { average. } & \text { max. } & \text { min. } \\ 0.27 & 0.31 & 0.21\end{array}$

p) Chemical composition :-

$\begin{array}{lrr}\text { Water } & 12.82 & - \\ \text { D. R. sugar } & 1.19 & 1.37 \\ \text { Starch } & 73.08 & 83.79 \\ \text { Crude fat } & 2.43 & 2.79 \\ \quad \text { " fibre } & 1.20 & 1.38 \\ \quad \text { " protein } & 8.05 & 9.23 \\ \text { Ash } & 1.27 & 1.46 \\ \text { Phosphoric acid } & 0.53 & 0.61\end{array}$

J) Production of Kagawa prefecture.

a) Percentage of full grown grains.- $85.05 \%$

b) External appearance and luster.—Nearly same as G).

c) Size of grains, (m.m.)

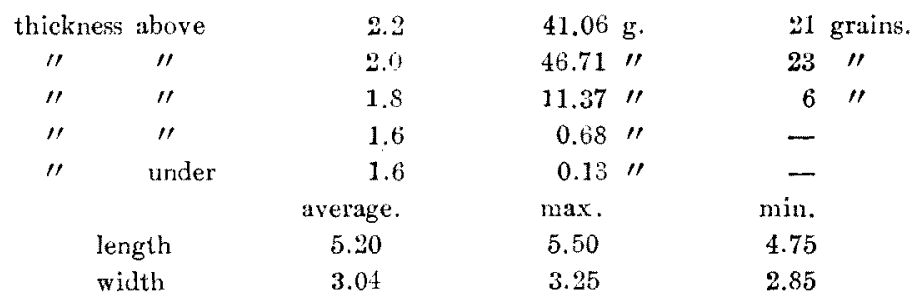

d) Percentage of Shinziromai._- $41.56 \%$

e) Depth of longitudinal furrow. $(\mu)$ 


$\begin{array}{rrr}\text { average. } & \max . & \min . \\ 56.23 & 95.15 & \mathbf{2 5 . 9 5}\end{array}$

f) Specific gravity. -1.409

g) Saccharifying quality. $-34.36 \%$

h) Hardnesss. (kilo)

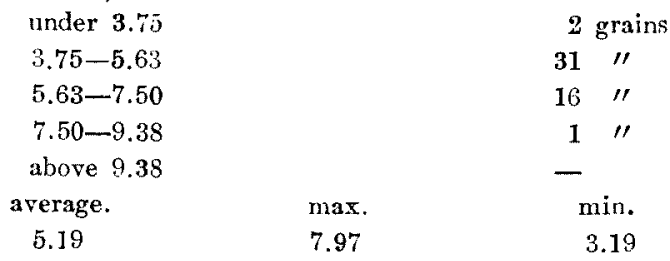

i) Volume of $100 \mathrm{~g}$. - 126.0c.c.

j) Weight of 1,000 grains. $-24.23 \mathrm{~g}$.

k) Volume of 1,000 grains. - 17.30c.c.

l) Phytin.- $0.43 \%$

m) Thickness of bran layer. ( $\mu$ )

$$
\begin{array}{ccc}
\text { average. } & \max . & \mathrm{min} . \\
45.88 & 49.95 & 42.08
\end{array}
$$

n) Absorption of water.

Time steeped.

$$
24 \mathrm{~h} \text {. }
$$

$\%$ absorbed.

25.34

$\min$.

0.17

p) Chemical composition :-

$\begin{array}{lrr}\text { Water } & 12.72 & - \\ \text { D. R. sugar } & 0.90 & 1.06 \\ \text { Starch } & 72.27 & 84.59 \\ \text { Crude fat } & 2.39 & 2.80 \\ \quad \text { " fibre } & 1.21 & 1.42 \\ \quad \text { " protein } & 7.46 & 8.73 \\ \text { Ash } & 1.21 & 1.42 \\ \text { Phosphoric acid } & 0.53 & 0.62\end{array}$

K) Production of Yelime prefecture.

a) Percentage of full grown grains. $-87.96 \%$

b) External appearance and luster.-Straw yellow waxy luster, grains

\begin{tabular}{|c|c|}
\hline Thic & $s$ above \\
\hline " & 'I \\
\hline "I & "I \\
\hline 'I & "' \\
\hline "I & under \\
\hline
\end{tabular}
were large and fat, impurities were perceptible.

c) Size of grains. (m.m.)

$62.45 \mathrm{~g}$.
$28.17 "$
$8.15 \prime \prime$
$0.92 "$
$0.19 \prime$




$\begin{array}{lcrc} & \text { average. } & \text { max. } & \min . \\ \text { length } & 5.41 & 5.90 & 4.95 \\ \text { width } & 3.02 & 3.25 & 2.70\end{array}$

d) Percentage of Shinziromai- $52.52 \%$

e) Depth of longitudinal furrow. $(\mu)$

$\begin{array}{ccc}\text { average. } & \max . & \min . \\ 51.47 & 86.50 & 17.30\end{array}$

f) Specific gravity.-1.399

g) Saccharifying quality, $-37.43 \%$

h) Hardness. (kilo)

\begin{tabular}{|c|c|c|}
\hline under 3.75 & & $3 \mathrm{grai}$ \\
\hline $3.75-5.63$ & & $29 \prime \prime$ \\
\hline $5.63-7.50$ & & 18 \\
\hline $7.50-9.38$ & & - \\
\hline above 9.38 & & - \\
\hline $\begin{array}{c}\text { average. } \\
5.26\end{array}$ & $\max$. & $\min$. \\
\hline
\end{tabular}

i) Volume of $100 \mathrm{~g} . \longrightarrow 124.0 \mathrm{c} . \mathrm{c}$.

j) Weight of 1,000 grains.-- $25.77 \mathrm{~g}$.

k) Volume of 1,000 grains. - 18.3c.c.

1) Phytin.- $0.43 \%$

m) Thickness of bran layer. $(\mu)$

$\begin{array}{ccc}\text { average. } & \max . & \min . \\ 44.79 & 49.95 & 40.73\end{array}$

n) Absorption of water.

time steeped. $\%$ absorbed.

$24 \mathrm{~b} . \quad 21.36$

o) Depth of embryo cavity :-

average. $\max$. $\min$.

$\begin{array}{lll}0.27 & 0.31 & 0.23\end{array}$

p) Chemical composition :-

$\begin{array}{lrr}\text { Water } & 13.46 & - \\ \text { D. R. sugar } & 0.83 & 0.96 \\ \text { Starch } & 73.04 & 84.59 \\ \text { Crude fat } & 2.31 & 2.68 \\ \quad \text { " fibre } & 1.04 & 1.20 \\ \quad \text { " protein } & 7.96 & 9.22 \\ \text { Ash } & 1.16 & 1.34 \\ \text { Phosphoric acid } & 0.51 & 0.59\end{array}$

I) Production of Yelime prefecture.

a) Percentage of full grown grains.- $89.48 \%$

b) External appearance and luster.-Nearly same as before.

c) Size of grains. $(\mathrm{m}, \mathrm{m}$.) 


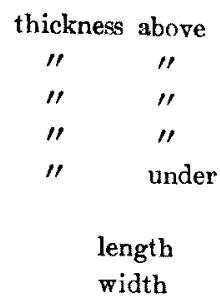

2.2
2.0
1.8
1.6
1.6
average.
5.44
3.03

$52.24 \mathrm{~g}$

36.25 "

$10.59 \prime \prime$

$0.75 \prime \prime$

$0.15 \prime \prime$

$\max$.

5.75

3.20

$\begin{aligned} 26 & \text { grains. } \\ 18 & \prime \prime \\ 5 & \prime \prime \\ 1 & \prime \prime \\ - & \\ \text { min. } & \\ 5.00 & \\ 2.75 & \end{aligned}$

d) Percentage of Shinziromai.-63.28\%

e) Depth of longitudinal furrow. $(\mu)$

$\begin{array}{ccc}\text { average. } & \max . & \min . \\ 50.60 & 86.50 & 34.60\end{array}$

f) Specific gravity. -1:409

g) Saccharifying quality. $-36.17 \%$

h) Hardness. (kilo)

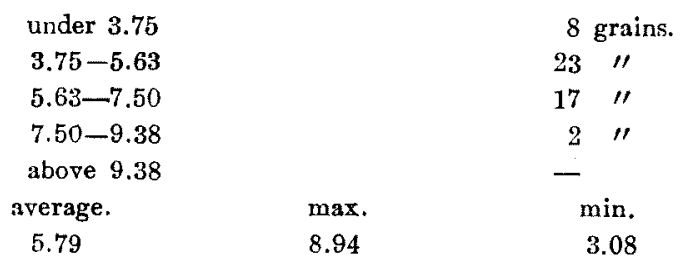

i) Volume of $100 \mathrm{~g}$ - 124.0c.c.

j) Weight of 1,000 grains. $-25.5 \mathrm{~g}$.

k) Volume of 1,000 grains._-18.2c.c.

l) Phytin. $-0.37 \%$

m) Thickness of bran layer. $(\mu)$
average.
$\max$.
$\min$.
44.46
50.18
38.25

n) Absorption of water.

Time steeped.

$24 \mathrm{~h}$.

$\%$ absorbed.

23.92

o) Depth of embryo cavity :$\begin{array}{cr}\text { average. } & \max . \\ 0.25 & 0.32\end{array}$

$\min$.

0.21

p) Chemical composition :-

$\begin{array}{lr}\text { Water } & 12.51 \\ \text { D. K. sugar } & 1.09 \\ \text { Starch } & 73.32 \\ \text { Crude fat } & 2.57 \\ \quad \prime \prime \text { fibre } & 1.48 \\ \quad \prime \prime \text { protein } & 7.71 \\ \text { Ash } & 1.21 \\ \text { Phosphoric acid } & 0.46\end{array}$

unhydrous.

2.51

1.09

3.32

2.57

1.48

7.71

0.46
1.25

83.91

2.94

1.69

8.82

1.38

0.53 
M) Production of Fukushima prefecture.

a) Percentage of full grown grains. $-89.4 \%$

h) External appearance and luster-Brownish straw yellow colour, destitute of luster, grains were not fat.

c) Size of grains. (m.m.)

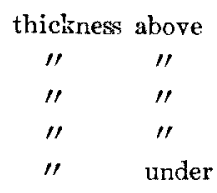

length

width

2.2
2.0
1.8
1.6
1.6
average.
5.03

3.01

$\begin{array}{rlr}56.54 & \text { g. } & 28 \text { grains. } \\ 30.86 \prime \prime & 15 \prime \prime \\ 11.12 \prime \prime & 6 " \prime \\ 1.09 \prime \prime & 1 & \prime \prime \\ 0.34 \prime \prime & - & \\ \max . & & \text { min. } \\ 5.55 & 4.65 \\ 3.25 & 2.65\end{array}$

$19.04 \%$

e) Depth of longitudinal furrow. $(\mu)$

$\begin{array}{ccc}\text { average } & \max . & \min . \\ 60.55 & 103.80 & 17.30\end{array}$

f) Specific gravity.- 1.418

g) Saccharifying quality._-37.53\%

h) Hardness. (kilo)

\begin{tabular}{|c|c|c|}
\hline under 3.75 & & 5 grains. \\
\hline $3.75-5.63$ & & $26 \prime \prime$ \\
\hline $5.63-7.50$ & & 19 \\
\hline $7.50-9.38$ & & - \\
\hline above 9.38 & & - \\
\hline average. & $\max$ & $\min$. \\
\hline 5.11 & 7.01 & 2.70 \\
\hline
\end{tabular}

i) Volume of $100 \mathrm{~g}$. - $130.0 \mathrm{c} . \mathrm{c}$.

j) Weight of 1,000 grains.-24.1g. 25.85g. (Shinziromai)

k) Volume of 1,000 grains., 17.1c.c. 18.35c.c. (Shinziromai)

1) Phytin.-0.49\%

m) Thickness of bran layer. $(\mu)$ $\begin{array}{rcr}\text { average. } & \max . & \min . \\ 45.61 & 49.95 & 41.63\end{array}$

n) Absorption of water. time steeped. $\%$ absorbed. $24 \mathrm{~h}$.

o) Depth of embryo cavity :-

$\begin{array}{ccc}\text { average. } & \max . & \min . \\ 0.26 & 0.33 & 0.20\end{array}$

p) Chemical composition :- 


$\begin{array}{lr}\text { Water } & 13.7 \\ \text { D. R. sugar } & 0.7 \\ \text { Starch } & 73.0 \\ \text { Crude fat } & 2.10 \\ \prime \prime \text { fibre } & 1.08 \\ \prime \prime \text { protein } & 7.4 \\ \text { Ash } & 1.28 \\ \text { l'hosphoric acid } & 0.49 \\ \text { of Fukuslima prefecture. }\end{array}$

unbydrous.

0.85

85.25

2.45

1.26

8.70

1.49

0.57

N) Production of Fukushima prefecture.

a) Percentage of full grown grains. $\quad 74.05 \%$

b) External appearance and luster.- Nearly same as M), but impurities were tolerably abundant.

c) Size of grains. (m.m.)

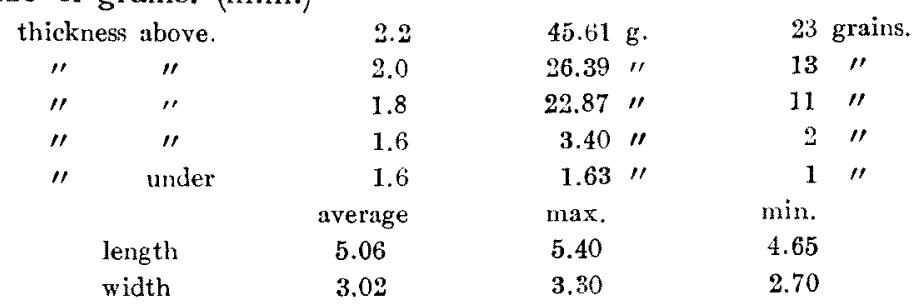

c) Percentage of Shinziromai- $-16.22 \%$

e) Depth of longitudinal furrow. $(\mu)$

$\begin{array}{ccr}\text { average. } & \max . & \min . \\ 49.95 & 86.50 & 17.30\end{array}$

f) Specific gravity.- 1.382

g) Saccharifying quality._- $34.88 \%$

h) Hardness. (kilo)

\begin{tabular}{|c|c|c|}
\hline under 3.75 & & $2 \mathrm{grai}$ \\
\hline $3.75-5.63$ & & $35 \prime \prime$ \\
\hline $5.63-7.50$ & & $13 \prime$ \\
\hline $7.50-9.38$ & & - \\
\hline above 9.38 & & - \\
\hline $\begin{array}{c}\text { average. } \\
5.18\end{array}$ & $\begin{array}{l}\max \\
6.56\end{array}$ & $\begin{array}{r}\text { min. } \\
3.56\end{array}$ \\
\hline
\end{tabular}

i) Volume of $100 \mathrm{~g}$ - $132.0 \mathrm{c} . \mathrm{c}$.

j) Weight of 1,000 grains. $-23.62 \mathrm{~g}$.

k) Volume of 1,000 grains.-17.1c.c.

m) Thickness of bran layer. $(\mu)$

$$
\begin{array}{ccc}
\text { average. } & \max . & \text { min. } \\
46.20 & 50.63 & 42.30
\end{array}
$$

n) Absorption of water.

time steeped. $\quad \%$ absorbed.

$2+\mathrm{h}$. 
o) Depth of embryo cavity :-

$\begin{array}{ccc}\text { average. } & \max . & \min . \\ 0.26 & 0.32 & 0.22\end{array}$

p) Chemical composition :-

$\begin{array}{lrc}\text { Water } & & \text { unhydrous. } \\ \text { D. R. sugar } & 14.54 & - \\ \text { Starch } & 1.38 & 1.63 \\ \text { Crude fat } & 71.91 & 84.77 \\ \quad \text { " fibre } & 2.14 & 2.53 \\ \quad \text { " protein } & 0.93 & 1.09 \\ \text { Ash } & 7.20 & 8.49 \\ \text { Phosphoric acid } & 1.27 & 1.50 \\ & 0.46 & 0.55\end{array}$

O) Production of Miyagi prefecture.

a) Percentage of full grown grains.-_- $86.2 \%$

b) External appearance and luster.-Nearly same as N), but impurities were somewhat rare.

c) Size of grains. (m.m.)

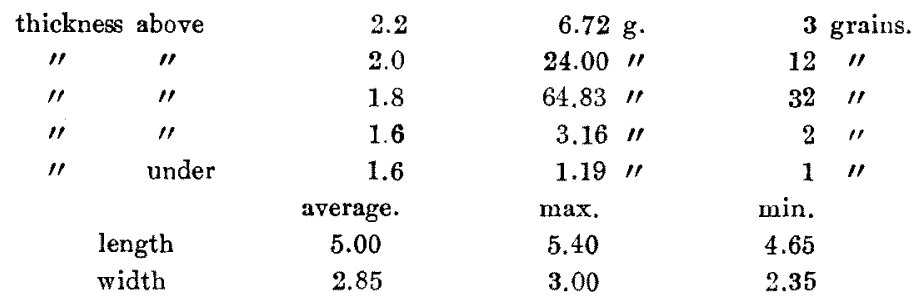

d) Percentage of Shinziromai.- $9.5 \%$

e) Depth of longitudinai furrow. ( $\mu$ )

$\begin{array}{rrr}\text { average. } & \max . & \min . \\ 55.15 & 86.50 & 34.60\end{array}$

f) Specific gravity.-1.394

g) Saccharifying quality.-28.04\%

h) Hardness. (kilo)

\begin{tabular}{|c|c|c|}
\hline under 3.75 & & 7 grains. \\
\hline $3.75-5.63$ & & $35 \quad " \prime$ \\
\hline $5.63-7.50$ & & " \\
\hline $7.50-9.38$ & & - \\
\hline above 9.38 & & - \\
\hline average. & $\max$. & min. \\
\hline 4.80 & 6.56 & 2.8 \\
\hline
\end{tabular}

i) Volume of $100 \mathrm{~g}$. 130.5 c.c.

j) Weight of 1,000 grains. $-20.47 \mathrm{~g}$.

k) Volume of 1,000 grains.-14.7c.c.

m) Thickness of bran layer. $(\mu)$ 

average.
42.74
$\max$.
48.15
$\min$.
35.78

n) Absorption of water.

time steeped.

$24 \mathrm{~h}$.

$\%$ absorbed.

20.76

o) Depth of embryo cavity :-

average.

$\max$.

min.

0.26

0.34

0.23

p) Chemical composition :-

$\begin{array}{lr}\text { Water } & 14.07 \\ \text { D. R. sugar } & 1.22 \\ \text { Starch } & 72.78 \\ \text { Crude fat } & 2.22 \\ \quad \text { " fibre } & 0.78 \\ \quad \text { " protein } & 7.62 \\ \text { Ash } & 1.23 \\ \text { Phosphoric acid } & 0.55\end{array}$

$$
\text { unhydrous. }
$$

$$
\begin{array}{r}
1.42 \\
84.78 \\
2.59 \\
0.91 \\
8.88 \\
1.44 \\
0.64
\end{array}
$$

P) Production of Niigata prefecture.

a) Percentage of full grown grains. $-92.42 \%$

b) External appearance and luster.—Straw yellow colour, somewhat destitute of luster, grains were not fat.

c) Size of grains. (m.m.)

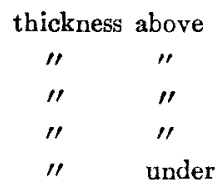

length

width

\begin{tabular}{|c|c|c|}
\hline uuder 3.75 & & 1 grains. \\
\hline $3.75-5.63$ & & $14 \prime \prime$ \\
\hline $5.63-7.50$ & & 35 \\
\hline $7.50-9.38$ & & - \\
\hline above 9.38 & & - \\
\hline $\begin{array}{c}\text { average. } \\
5.90\end{array}$ & $\begin{array}{l}\max . \\
7.39\end{array}$ & $\begin{array}{r}\min . \\
3.33\end{array}$ \\
\hline
\end{tabular}

$\begin{array}{cc}31.94 \mathrm{~g} . & 16 \text { grains. } \\ 52.42 \prime \prime & 26 \prime \prime \\ 14.48 \prime \prime & 8 \prime \prime \\ 0.60 \prime \prime & - \\ 0.13 \prime \prime & - \\ \max . & \operatorname{min.} \\ 5.65 & 4.90 \\ 3.15 & 2.75\end{array}$

d) Percentage of Shinziromai. $-10.5 \%$

e) Depth of longitudinal furrow. $(\mu)$

$\begin{array}{ccc}\text { average. } & \max . & \min . \\ 51.04 & 69.20 & 17.30\end{array}$

f) Specific gravity.- -1.409

g) Saccharifying quality. - $35.48 \%$

h) Hardness. (kilo) 
i) Volume of 100g.—128.0c.c.

j) Weight of 1,000 grains. $-23.75 \mathrm{~g}$.

k) Volume of 1,000 grains._-17.0c.c.

l) Phytin.-0.44\%

m) Thickness of bran layer. $(\mu)$

$$
\begin{array}{ccc}
\text { average. } & \max . & \min . \\
45.00 & 51.98 & 40.73
\end{array}
$$

n) Absorption of water.

Time steeped.

$\%$ absorbed.

$24 \mathrm{~h}$.

22.26

o) Depth of embryo cavity :-

$\begin{array}{ccc}\text { average. } & \text { max. } & \min . \\ 0.23 & 0.27 & 0.19\end{array}$

p) Chemical composition :-

$\begin{array}{lrr}\text { Water } & 13.29 & - \\ \text { D. R. sugar } & 1.79 & 2.10 \\ \text { Starch } & 71.61 & 83.75 \\ \text { Crude fat } & 2.39 & 2.78 \\ \quad \text { " fibre } & 0.92 & 1.08 \\ \text { " protein } & 7.62 & 8.91 \\ \text { Ash } & 1.18 & 1.38 \\ \text { Phosphoric acid } & 0.42 & 0.50\end{array}$

Q) Production of Aichi prefecture.

a) Percentage of full grown grains.-66.77\%

b) External appearance and luster.-Greyish straw yellow colour, grains were not fat, no luster, impurities were abundant.

\begin{tabular}{|c|c|c|c|c|c|c|}
\hline thick & above & 2.2 & 23.57 & & 12 & grains. \\
\hline $1 \prime$ & $\prime \prime$ & 2.0 & 39.58 & $" \prime$ & 20 & $"$ \\
\hline$\prime \prime$ & $\prime \prime$ & 1.8 & 28.39 & $\prime \prime$ & 14 & $" \prime$ \\
\hline$" \prime$ & $" r$ & 1.6 & 6.40 & $" \prime$ & 3 & $\prime \prime$ \\
\hline " & under & 1.6 & 1.97 & $" \prime$ & 1 & " \\
\hline & & average. & $\max$. & & min. & \\
\hline & gth & 5.08 & 5.50 & & 4.30 & \\
\hline & th & 2.95 & 3.20 & & 2.60 & \\
\hline
\end{tabular}

c) Size of grains. (m.m.)

d) Percentage of Shinziromai.- $30.5 \%$

e) Depth of longitudinal furrow. $(\mu)$

$\begin{array}{ccc}\text { average } & \max . & \text { min. } \\ 64.01 & 103.80 & 34.60\end{array}$

f) Specific gravity.-1.382

g) Saccharifying quality.-28.34\%

h) Hardness. (kilo) 


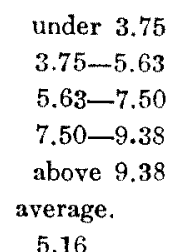

under 3.75

above 9.38

5.16

i) Volume of $100 \mathrm{~g}$ - 130.0c.c.

j) Weight of 1,000 grains. $-22.5 \mathrm{~g}$.

k) Volume of 1,000 grains.-16.2c.c.

$\mathrm{m})$ Thickness of bran layer. $(\mu)$ average. 45.99 n) Absorption of water.

Time steeped.

$24 \mathrm{~h}$.

o) Depth of embryo cavity :-

$$
\begin{gathered}
\text { average. } \\
0.26
\end{gathered}
$$

p) Chemical composition :-
Water
D. R. sugar

Starch

Crude fat

"I fibre

" protein

Ash

Phosphoric acid
13.91

1.92

70.27

2.34

1.20

8.29

1.29

0.38
5 grains.

2511

$20 " 1$

-

$\min$.

2.87
$\%$ absorbed.

25.6

min.

0.30

unhydrous.
2.26

82.37

2.74

1.41

9.71

1.51

0.45

R) Production of Miye prefecture.

a) Percentage of full grown grains. - $76.5 \%$

b) External appearance and luster.—Nearly same as $Q$ ).

c) Size of grains. (m.m.)

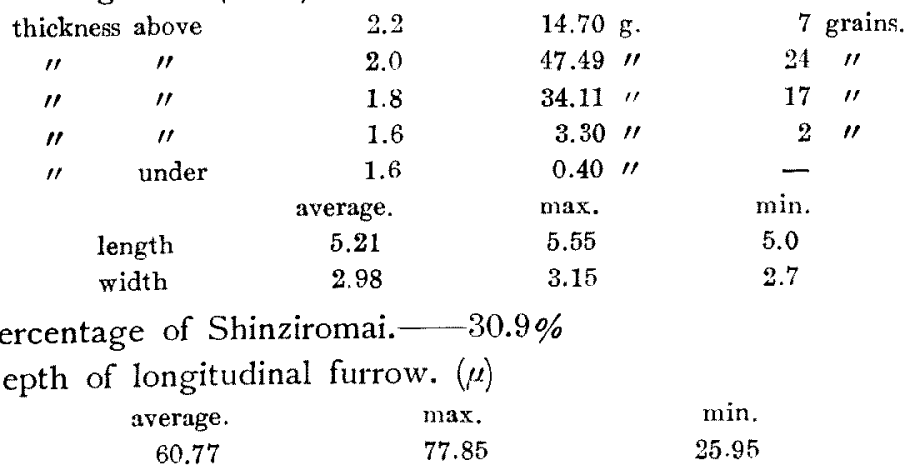

d) Percentage of Shinziromai.- $30.9 \%$

e) Depth of longitudinal furrow. $(\mu)$
60.77
77.85 
f) Specific gravity. 1.394

g) Saccharifying quality.—34.84\%

h) Hardness. (kilo)

$\begin{array}{lcc}\text { under } 3.75 & & 1 \text { grains } \\ 3.75-5.63 & & 26 \quad " \\ 5.63-7.50 & & 23 \quad \prime \\ 7.50-9.38 & & - \\ \text { above } 9.38 & & - \\ \text { average. } & \text { max. } & \text { min. } \\ 5.44 & 7.01 & 3.53\end{array}$

i) Volume of $100 \mathrm{~g}$ - 125.5c.c.

j) Weight of 1,000 grains. $22.85 \mathrm{~g}$.

k) Volume of 1,000 grains.-16.5c.c.

m) Thickness of bran layer. $(\mu)$

$\begin{array}{ccc}\text { average. } & \max . & \min . \\ 46.88 & 52.73 & 42.98\end{array}$

n) Absorption of water.

time steeped. $\quad \%$ absorbed.

$$
24 \mathrm{~b} \text {. } \quad 25.32
$$

o) Depth of embryo cavity :-

$\begin{array}{ccc}\text { average. } & \max . & \min . \\ 0.25 & 0.31 & 0.18\end{array}$

p) Chemical composition :-

$\begin{array}{lrr}\text { Water } & 13.07 & - \\ \text { D. R. sugar } & 1.42 & \mathbf{1 . 6 3} \\ \text { Starch } & 72.66 & 83.84 \\ \text { Crude fat } & 2.48 & 2.87 \\ \quad \text { " fibre } & 0.85 & 0.98 \\ \quad \text { " protein } & 7.88 & 9.09 \\ \text { Ash } & 1.48 & 1.71 \\ \text { Phosphoric acid } & 0.48 & 0.56\end{array}$

S) Production of Okayama prefecture.

a) Percentage of full grown grains. $-89.25 \%$

b) Exteral appearance luster.-Light straw yellow waxy luster, grains were fat and large.

c) Size of grains. (m.m.)

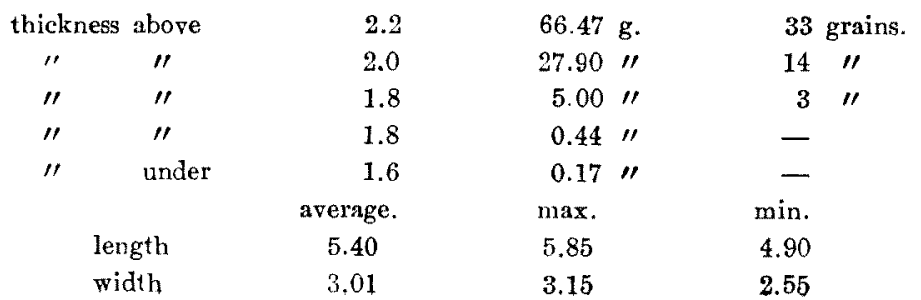


d) Percentage of Shinziromai.- $42.7 \%$

e) Depth of longitudinal furrow. $(\mu)$

$\begin{array}{rrr}\text { average. } & \max . & \min . \\ 51.47 & 69.00 & 25.95\end{array}$

f) Specific gravity.- 1.399

g) Saccharifying quality.-35.04\%

h) Hardness. (kilo)

\begin{tabular}{|c|c|c|}
\hline under 3.75 & & 11 grains \\
\hline $3.75-5.63$ & & 32 \\
\hline $5.63-7.50$ & & 7 \\
\hline $7.50-9.38$ & & - \\
\hline above 9.38 & & - \\
\hline average. & $\max$. & $\begin{array}{l}\min . \\
2.51\end{array}$ \\
\hline
\end{tabular}

i) Volume of $100 \mathrm{~g}$. - 124.5c.c.

j) Weight of 1,000 grains.- $26.52 \mathrm{~g}$.

k) Volume of 1,000 grains.-19.0c.c.

1) Phytin. - $0.38 \%$

m) Thickness of bran layer. $(\mu)$

$\begin{array}{ccc}\text { average. } & \max . & \min . \\ 48.54 & 56.48 & 42.53\end{array}$

n) Absorption of water.

Time steeped. $\%$ absorbed.

$24 \mathrm{~h} . \quad 24.02$

o) Depth of embryo cavity:-

$\begin{array}{ccc}\text { average. } & \max . & \min . \\ 0.25 & 0.31 & 0.18\end{array}$

p) Chemical composition :-

$\begin{array}{lrr}\text { Water } & 13.04 & - \\ \text { D. R. sugar } & 1.22 & 1.40 \\ \text { Starch } & 73.29 & 84.52 \\ \text { Crude fat } & 2.16 & 2.49 \\ \quad \text { "fibre } & 1.50 & 1.73 \\ \quad \text { " protein } & 7.46 & 8.60 \\ \text { Ash } & 1.10 & 1.27 \\ \text { Phosphoric acid } & 0.46 & 0.53\end{array}$

T) Production of Hiroslima prefecture.
a) Percentage of full grown grains. $-85.15 \%$
b) External appearance and luster.-Nearly same as S).
c) Size of grains. (m.m.) 


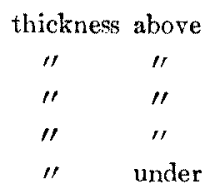

length width

2.2
2.0
1.8
1.6
1.6
average.
5.36
3.07

$60.25 \mathrm{~g}$.
$29.83 " \prime$
$9.24 " \prime$
$0.56 "$
$0.11 "$
$\max$.
5.70
3.25

30 grains.

15 "

$5 \quad 11$

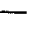

min.

4.95

2.90

d) Percentage of Shinziromai.- $59.0 \%$

e) Depth of longitudinal furrow. ( $/ 1)$

$\begin{array}{clc}\text { average. } & \max . & \mathrm{min} . \\ 55.79 & 103.80 & 34.60\end{array}$

f) Specific gravity.-1.409

g) Saccharifying quality.-38.32\%

h) Hardness. (kilo)

\begin{tabular}{|c|c|c|c|}
\hline under 3.75 & & & rains. \\
\hline $3.75-5.63$ & & 27 & $\prime \prime$ \\
\hline $5.63-7.50$ & & 12 & $\prime \prime$ \\
\hline $7.50-9.38$ & & 3 & $"$ \\
\hline above 9.38 & & - & \\
\hline average. & $\max$. & & in. \\
\hline 5.17 & 7.88 & & .81 \\
\hline
\end{tabular}

i) Volume of $100 \mathrm{~g} . \longrightarrow 123.0 \mathrm{c} . \mathrm{c}$.

j) Weight of 1,000 grains. - 25.95g.

k) Volume of 1,000 grains.-18.5c.c.

1) Phytin. $-0.38 \%$

m) Thickness of bran layer. $(k)$

$$
\begin{array}{ccc}
\text { average. } & \max . & \min . \\
46.41 & 51.08 & 40.40
\end{array}
$$

n) Absorption of water.

time steeped. $\%$ absorbed.

$$
24 \mathrm{~h} . \quad 24.46
$$

o) Depth of embryo cavity :-

$\begin{array}{ccc}\text { average. } & \max . & \min . \\ 0.26 & 0.33 & 0.12\end{array}$

p) Chemical composition :-

$\begin{array}{lr}\text { Water } & 13.10 \\ \text { Direct R. sugar } & 0.94 \\ \text { Starch } & 73.03 \\ \text { Crude fat } & 2.27 \\ \quad \text { " fibre } & 1.10 \\ \text { "' protein } & 7.46 \\ \text { Ash } & 1.12 \\ \text { Phosphoric acid } & 0.45\end{array}$

unhydrous. 
B) Polished rice, suitable for the Sake brewing. 20 (A-T) varieties.

A) Production of Okayama prefecture.

a) Percentage of full grown grains.-97.35\%.

b) External appearance and luster._White glassy luster, grains were fat and large.

c) Size of grains. (m.m.)

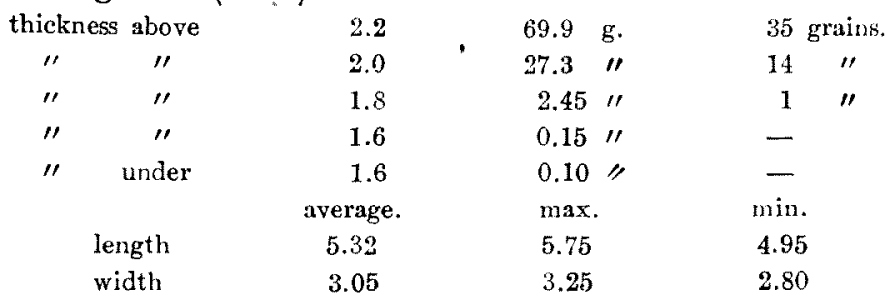

d) Percentage of Shinziromai._- $84.42 \%$

e) Depth of longitudinal furrow. $(\mu)$

$$
\text { average. } \max \text {. min. }
$$

$\begin{array}{lll}34.82 & 69.20 & 8.65\end{array}$

f) Specific gravity.-1.429

g) Saccharifying quality.—22.71\%

h) Hardness. (kilo)

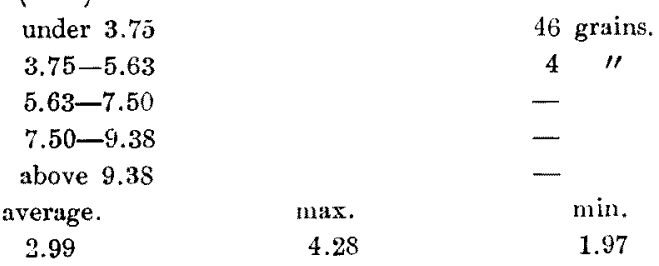

i) Volume of $100 \mathrm{~g} .-120.0$ c.c.

j) Weight of 1,000 grains. $-25.45 \mathrm{~g}$.

k) Volume of 1,000 grains. 18.8c.c.

l) Phytin.- $0.02 \%$

n) Absorption of water.

tine steeped.

$$
94 \mathrm{~h} \text {. }
$$

\% absorbed.

19.9

p) Chemical composition :-

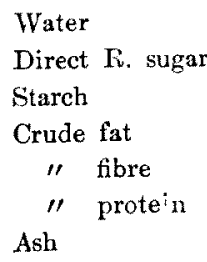

13.27

0.46

78.28

0.39

0.45

6.69

0.30

Phosphoric acid

0.18 unh ydrous.

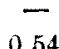

c0.59

0.34

0.51

7.74

0.35

0.21 
B) Production of Okayama prefecturc.

a) Percentage of full grown grains.- $96.73 \%$

b) External appearance and Iuster._- Same as A).

c) Size of grains. (m.m.)

\begin{tabular}{|c|c|c|c|c|c|}
\hline \multicolumn{2}{|c|}{ hickness above } & 2.2 & 48.1 & \multicolumn{2}{|c|}{24 grains } \\
\hline$" \prime$ & $" \prime$ & 2.0 & 42.15 & 21 & $"$ \\
\hline$"$ & $" \prime$ & 1.8 & 9.25 & 5 & " \\
\hline$" \prime$ & $" \prime$ & 1.6 & 0.42 & - & \\
\hline " & under & 1.6 & 0.05 & - & \\
\hline & length & $\begin{array}{c}\text { average. } \\
5.21\end{array}$ & $\begin{array}{l}\max . \\
5.65\end{array}$ & $\begin{array}{l}\min . \\
4.70\end{array}$ & \\
\hline & width & 2.95 & 3.15 & 2.55 & \\
\hline
\end{tabular}

d) Percentage of Shinziromai._-63.28\%

e) Depth of longitudinal furrow. $(\mu)$

$\begin{array}{rcc}\text { average. } & \max . & \min . \\ 27.64 & 51.90 & 5.19\end{array}$

f) Specific gravity.--1.449

g) Saccharifying quality. $-24.64 \%$

h) Hardness. (kilo)

$\begin{array}{lcc}\text { under } 3.75 & & 43 \text { grains. } \\ 3.75-5.63 & & 7 \\ 5.63-7.50 & & - \\ 7.50-9.38 & & - \\ \text { above } 9.38 & & - \\ \text { average. } & \text { max. } & \text { min. } \\ 2.91 & 4.65 & 1.97\end{array}$

i) Volume of $100 \mathrm{~g} . \longrightarrow 119.0 \mathrm{c} . \mathrm{c}$.

j) Weight of 1,000 grains. $-23.75 \mathrm{~g}$.

k) Volume of 1,000 grains. - 16.6c.c.

l) Phytin.-0.06\%

n) Absorption of water.

time steeped.

$2 \mathrm{~h}$.

$\%$ absorbed

20.08

p) Chemical composition :-

$\begin{array}{lrr}\text { Water } & 13.22 & - \\ \text { Direct, R. sugar } & 0.54 & 0.62 \\ \text { Starch } & 78.22 & 90.30 \\ \text { Crude fat } & 0.37 & 0.43 \\ \quad \text { " fibre } & 0.62 & 0.72 \\ \quad \text { " protein } & 6.53 & 7.54 \\ \text { Ash } & 0.35 & 0.40 \\ \text { Phosphoric acid } & 0.18 & 0.21\end{array}$


C) Production of Niigata prefecture.

a) Percentage of full grown grains._- $94.58 \%$

b) External appearance and luster.—Slightly brownish glassy luster, grains were not fat and of middle size.

c) Size of grains. (m.m.)

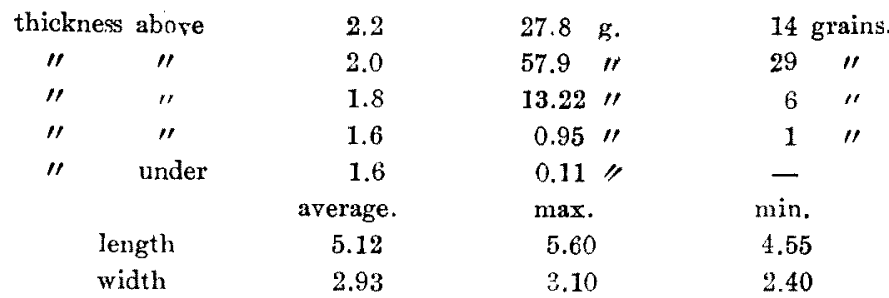

d) Percentage of Shinziromai.-16.4\%

e) Depth of longitudinal furrow. $(\mu)$

$\begin{array}{rlr}\text { average. } & \max . & \min . \\ 34.38 & 51.90 & 17.30\end{array}$

f) Specific gravity.-1.429

g) Saccharifying quality.-29.48\%

h) Hardness. (kilo)

$\begin{array}{ccc}\text { under } 3.75 & & 50 \text { grains. } \\ 3.75-5.63 & & - \\ 5.63-7.50 & & - \\ 7.50-9.38 & & - \\ \text { above } 9.38 & & \text { min. } \\ \text { average. } & \text { max. } & 1.97 \\ 2.56 & 3.38 & \end{array}$

i) Volume of $100 \mathrm{~g}$. - $120.5 \mathrm{c} . \mathrm{c}$.

j) Weight of 1,000 grains. $-21.8 \mathrm{~g}$.

k) Volume of 1,000 grains. - 15.3c.c.

n) Absorption of water.

time steeped.

2 h.'

$\%$ absorbed.

16.66

p) Chemical composition :-

$\begin{array}{lrr}\text { Water } & 14.05 & - \\ \text { D. R. sugar } & 0.50 & 0.58 \\ \text { Starch } & 77.24 & 89.90 \\ \text { Crude fat } & 0.29 & 0.33 \\ \quad \text { " fibre } & 0.31 & 0.36 \\ \quad \text { " protein } & 7.29 & 8.48 \\ \text { Ash } & 0.29 & 0.34 \\ \text { Phosphoric acid } & 0.18 & 0.20\end{array}$


D) Production of IIyogo prefecture.

a) Percentage of full grown grains. $\longrightarrow 93.9 \%$.

b) External appearance and luster.—- nearly same as A).

c) Size of grains. (m.m.)

\begin{tabular}{|c|c|c|c|c|c|}
\hline \multicolumn{2}{|c|}{ thickness above } & 2.2 & $14.22 \mathrm{~g}$. & \multicolumn{2}{|c|}{7 grain } \\
\hline$\prime \prime$ & $\prime \prime$ & 2.0 & $51.51 "$ & 26 & " \\
\hline$\prime \prime$ & $" \prime$ & 1.8 & $33.45 \prime \prime$ & 17 & " \\
\hline$\prime \prime$ & $" \prime$ & 1.6 & $0.82 \prime \prime$ & - & \\
\hline , & under & 1.6 & - & - & \\
\hline & & average. & $\max$. & min. & \\
\hline & gth & 5.23 & 5.65 & 4.80 & \\
\hline & dth & 3.01 & 3.25 & 2.70 & \\
\hline
\end{tabular}

d) Percentage of Shinziromai._- $85.0 \%$

e) Depth of longitudinal furrow. $(\mu)$

$\begin{array}{ccc}\text { average. } & \text { max. } & \text { min. } \\ 46.49 & 69.20 & 17.30\end{array}$

f) Specific gravity.- 1.449

g) Saccharifying quality. $-19.22 \%$

h) Hardness. (kilo)

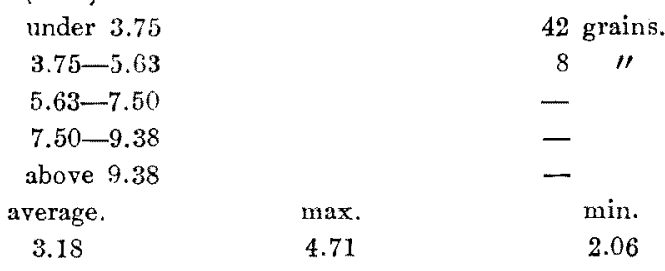

i) Volume of $100 \mathrm{~g}$. - $-116.0 \mathrm{c} . \mathrm{c}$.

j) Weight of 1,000 grains. $\_22.39 \mathrm{~g}$.

$24.29 \mathrm{~g}$. (Shinziromai)

k) Volume of 1,000 grains.—15.4c.c.

16.9c.c. (Shinziromai)

1) Phytin.- $0.03 \%$

n) Absorption of water.

time steeped.

$$
2 \mathrm{~h} .
$$

p) Chemical composition :-

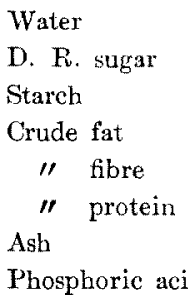

$\%$ absorbed.

20.86

unbydrous. 
E) Production of Hyogo prefecture.

a) Percentage of full grown grains. $-94.16 \%$

b) External appearance and luster._-Same as before.

c) Size of grains. (m.m.)

\begin{tabular}{|c|c|c|c|c|c|}
\hline hicks & above & 2.2 & $23.45 \mathrm{~g}$. & 12 & rains. \\
\hline " & $\prime \prime$ & 2.0 & $47.55 \prime \prime$ & 24 & $" \prime$ \\
\hline " & " & 1.8 & 28.18 & 14 & " \\
\hline$"$ & " & 1.6 & $0.72 \prime \prime$ & - & \\
\hline$\prime \prime$ & under & 1.6 & - & - & \\
\hline & gth & $\begin{array}{c}\text { average. } \\
5.15\end{array}$ & $\begin{array}{l}\max . \\
5.55\end{array}$ & $\begin{array}{r}\min , \\
4.75\end{array}$ & \\
\hline & dth & 2.98 & 3.15 & 2.70 & \\
\hline
\end{tabular}

d) Percentage of Shinziromai._- $74.48 \%$

e) Depth of longitudinal furrow. $(\mu)$

$\begin{array}{ccc}\text { average. } & \max . & \min . \\ 41.95 & 77.85 & 17.30\end{array}$

f) Specific gravity.-1.449\%.

g) Saccharifying quality. $21.68 \%$

h) Hardness. (kilo)

$\begin{array}{lrl}\text { under } 3.75 & & 40 \text { grains. } \\ 3.75-5.63 & & 10 " \\ 5.63-7.50 & & - \\ 7.50-9.38 & & - \\ \text { above } 9.38 & & - \\ \text { average. } & \text { max. } & \text { min. } \\ \mathbf{3 . 2 4} & 4.69 & 2.03\end{array}$

i) Volume of $100 \mathrm{~g} \cdot-113.5 \mathrm{c} . \mathrm{c}$.

j) Weight of 1,000 grains. $-22.68 \mathrm{~g}$.

k) Volume of 1,000 grains.-15.9c.c.

n) Absorption of water.

time steeped.

$2 \mathrm{~h}$.
$\%$ absorbed.

20.3

unhydrous.

13.06

0.40

78.92

0.16

0.35

6.61

0.27

0.17
0.46

90.92

0.18

0.40

7.62

0.31

0.20

Phosphoric acid 
F) Production of Hyogo prefecture.

a) Percentage of full grown grains.—94.62\%

b) External appearance and luster._-Nearly same as before.

c) Size of grains. (m.m.)

\begin{tabular}{|c|c|c|c|c|c|}
\hline \multicolumn{2}{|c|}{ thickness above } & 2.2 & $35.45 \mathrm{~g}$. & \multicolumn{2}{|c|}{18 grains. } \\
\hline$\prime \prime$ & $\prime \prime$ & 2.0 & $44.4 \prime \prime$ & 22 & $\prime \prime$ \\
\hline$\prime \prime$ & " & 1.8 & $19.6 \quad \prime \prime$ & 10 & $\prime \prime$ \\
\hline$" \prime$ & 11 & 1.6 & $0.55 / \prime$ & - & \\
\hline$" \prime$ & under & 1.6 & - & - & \\
\hline & & average. & $\max$. & $\min$. & \\
\hline & ath & 5.16 & 5.55 & 4.70 & \\
\hline & dth & 2.99 & 3.25 & 2.70 & \\
\hline
\end{tabular}

d) Percentage of Shinziromai._-54.56\%

e) Depth of longitudinal furrow. $(\mu)$

$\begin{array}{ccc}\text { average. } & \max . & \min . \\ 48.22 & 77.85 & 25.95\end{array}$

f) Specific gravity.-1.439

g) Saccharifying quality. $-17.27 \%$

h) Hardness. (kilo)

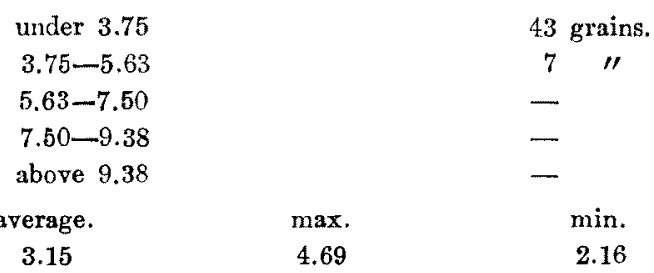

i) Volume of $100 \mathrm{~g} . \longrightarrow 112.0 \mathrm{c} . \mathrm{c}$.

j) Weight of 1,000 grains. $-23.0 \mathrm{~g}$.

k) Volume of 1,000 grains.-16.25c.c.

n) Absorption of water.

time steeped.

$$
2 \mathrm{~h} \text {. }
$$

p) Chemical composition :-

Water
D. R. sugar
Starch
Crude fat
$\quad$ " fibre
" protein
Ash

Phosphoric acid
13.48

0.41

78.62

0.20

0.39

6.44

0.27

0.16
$\%$ absorbed.

$\mathbf{1 9 . 6}$

unhydrous.
0.47
91.07
0.23
0.45
7.46
0.32
0.19 
G) Production of Fukwoka prefecture.

a) Percentage of full grown grains.- $-79.33 \%$

b) External appearance and luster.-_ Slightly brown white colour, destitute of luster, grains were not fat and of middle size.

c) Size of grains. (m.m.)

\begin{tabular}{|c|c|c|c|c|c|}
\hline \multicolumn{2}{|c|}{ thickness above } & 2.2 & $12.45 \mathrm{~g}$. & \multicolumn{2}{|c|}{6 grains. } \\
\hline " & $" \prime$ & 2.0 & $41.49 "$ & 21 & $" \prime$ \\
\hline$"$ & " & 1.8 & $40.45 \prime \prime$ & 20 & $" \prime$ \\
\hline " & $" \prime$ & 1.6 & $4.20 " \prime$ & 2 & $"$ \\
\hline " & under & $\begin{array}{c}1.6 \\
\text { average. }\end{array}$ & $\begin{array}{l}1.36 " \prime \\
\text { max. }\end{array}$ & $\begin{array}{c}1 \\
\min .\end{array}$ & $"$ \\
\hline & ngth & 5.04 & 5.40 & 4.55 & \\
\hline & $d t$ & 2.95 & 3.15 & 2.70 & \\
\hline
\end{tabular}

d) Percentage of Shinziromai.- $24.44 \%$

e) Depth of longitudinal furrow. $(\mu)$

$\begin{array}{ccc}\text { average. } & \text { max. } & \min . \\ 46.71 & 69.20 & 17.30\end{array}$

f) Specific gravity.-1.439

g) Saccharifying quality. $19.88 \%$

h) Hardness. (kilo)

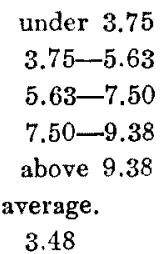

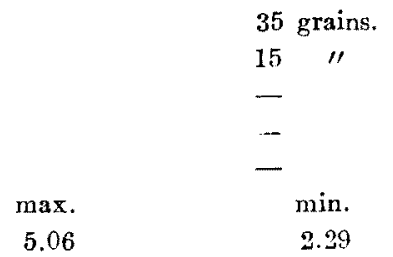

i) Volume of $100 \mathrm{~g} .-120.5 \mathrm{c} . \mathrm{c}$.

j) Weight of 1,000 grains. $-21.19 \mathrm{~g}$.

k) Volume of 1,000 grains.—15.0c.c.

1) Phytin. $-0.07 \%$

n) Absorption of water. time steeped.

$2 \mathrm{~h}$.

$\%$ absorbed.

19.52

p) Chemical composition.-

Water
D. R. sugar
Starch
Crude fat
$\prime \prime$ fibre
$\prime \prime$ protein
Ash
Phosphoric aci

12.97

0.30

77.27

0.25

0.54

7.62

0.52

0.27 unhydrous.

0.35

89.33

0.29

0.62

8.82

0.60

0.31 
H) Production of Kumamoto prefecture.

a) Percentage of full grown grains.- $82.57 \%$,

b) External appearance and luster.— Nearly same as G).

c) Size of grains. (m.m.)

\begin{tabular}{|c|c|c|c|c|c|}
\hline thickness & above & 2.2 & $37.92 \mathrm{~g}$. & \multicolumn{2}{|c|}{19 grains } \\
\hline$\prime \prime$ & $1 \prime$ & 2.0 & $52.00 " \prime$ & 26 & $\prime \prime$ \\
\hline$\prime \prime$ & "I & 1.8 & $6.33 \prime \prime$ & 3 & "I \\
\hline$\prime \prime$ & " & 1.6 & $3.40 \prime \prime$ & 2 & $\prime \prime$ \\
\hline "l & under & 1.6 & $0.34 \prime \prime$ & - & \\
\hline & & average. & $\max$. & $\min$. & \\
\hline & gth & 5.07 & 5.25 & 4.45 & \\
\hline & & 2.99 & 3.15 & 2.60 & \\
\hline
\end{tabular}

d) Percentage of Shinziromai.-34.8\%

e) Depth of longitudinal furrow. $(\mu)$

$\begin{array}{clr}\text { average. } & \max . & \min . \\ 34.17 & 51.90 & 8.65\end{array}$

f) Specific gravity.-1.439.

g) Saccharifying quality.-29.73\%

h) Hardness. (kilo)

$\begin{array}{llll}\text { under. } 3.75 & & 32 \text { grains. } \\ 3.75-5.63 & & 18 & \prime \prime \\ 5.63-7.50 & & - & \\ 7.50-9.38 & & - & \\ \text { above } 9.38 & & - & \\ \text { average. } & \text { max. } & & \mathrm{min} . \\ 3.54 & 5.25 & & 2.12\end{array}$

i) Volume of $100 \mathrm{~g} .-121.0 \mathrm{c} . \mathrm{c}$.

j) Weight of 1,000 grains. $-20.30 \mathrm{~g}$.

k) Volume of 1,000 grains.-14.9c.c.

1) Phytin. $-0.04 \%$

n) Absorption of water.

time steeped.

$$
2 \mathrm{~h} \text {. }
$$

\% absorbed.

20.40

p) Chemical composition.-

$\begin{array}{lr}\text { Water } & 12.82 \\ \text { D. R. sugar } & 0.25 \\ \text { Starch } & 78.92 \\ \text { Crude fat } & 0.15 \\ \quad \text { " fibre } & 0.22 \\ \quad \text { " protein } & 7.04 \\ \text { Ash } & 0.42 \\ \text { Phosphoric acid } & 0.17\end{array}$

unhydrous.
0.29
90.73
0.17
0.25
8.09
0.48
0.20 
I) Production of Saga prefecture.

a) Percentage of full grown grains. $-87.01 \%$

b) External appearance and luster.-Nearly same as A).

c) Size of grains. (m.m.)

\begin{tabular}{|c|c|c|c|c|c|}
\hline \multicolumn{2}{|c|}{ thickness above } & 2.2 & $43.55 \mathrm{~g}$. & \multicolumn{2}{|c|}{22 grains. } \\
\hline$\prime \prime$ & $"$ & 2.0 & $44.05 "$ & 22 & $\prime \prime$ \\
\hline$\prime \prime$ & " & 1.8 & $11.43 \prime \prime$ & 6 & $\prime \prime$ \\
\hline$" \prime$ & " & 1.6 & $0.52 " \prime$ & - & \\
\hline "I & under & 1.6 & $0.35 \prime \prime$ & - & \\
\hline & & average. & $\max$ & $\min$. & \\
\hline & length & 4.96 & 5.25 & 4.60 & \\
\hline & width & 3.01 & 3.15 & 2.70 & \\
\hline
\end{tabular}

d) Percentage of Shinziromai-_-35.5\%

e) Depth of longitudinal furrow. $(\mu)$

$\begin{array}{rcr}\text { average. } & \max . & \min . \\ 30.49 & 51.90 & 17.30\end{array}$

f) Specific gravity. 1.449

g) Saccharifying quality.-19.71\%

h) Hardness. (kilo)

\begin{tabular}{|c|c|c|}
\hline under 3.75 & & 32 grain \\
\hline $3.75-5.63$ & & $18 \quad \prime \prime$ \\
\hline $5.63-7.50$ & & - \\
\hline $7.50-9.38$ & & - \\
\hline above 9.38 & & - \\
\hline average. & $\max$. & min. \\
\hline 3.51 & 50.1 & 19.34 \\
\hline
\end{tabular}

i) Volume of $100 \mathrm{~g} . \longrightarrow 119.0 \mathrm{c} . \mathrm{c}$.

j) Weight of 1,000 grains. $-22.73 \mathrm{~g}$.

k) Volume of 1,000 grains. - 16.0c.c.

1) Phytin. $\quad 0.02 \%$

n) Absorption of water. time steeped.

2 h.

$\%$ absorbed

19.34

p) Chemical composition :-

unhydrous

$\begin{array}{lr}\text { Water } & 12.58 \\ \text { D. R. sugar } & 0.46 \\ \text { Starch } & 78.96 \\ \text { Crude fat } & 0.13 \\ \quad \text { " fibre } & 0.22 \\ \quad \prime \prime \text { protein } & 7.29 \\ \text { Ash } & 0.38 \\ \text { Phosphoric acid } & 0.24\end{array}$

-

0.53

90.93

0.15

0.25

8.34

0.42

0.28 
J) Production of Kagawa prefecture.

a) Percentage of full grown grains.-90.87\%

b) External appearance and luster.—Greyish white colour, destitute of luster, grains were fat and of middle size.

c) Size of grains. (m.m.)

\begin{tabular}{|c|c|c|c|c|c|}
\hline thicknes & above & 2.2 & $32.38 \mathrm{~g}$. & \multicolumn{2}{|c|}{16 graius. } \\
\hline " & $" \prime$ & 2.0 & $50.97 \prime \prime$ & 26 & $\prime \prime$ \\
\hline$\prime \prime$ & " & 1.8 & $16.40 " \prime$ & 8 & $"$ \\
\hline$" \prime$ & $\prime \prime$ & 1.6 & $0.24 \quad \prime \prime$ & - & \\
\hline " & under & 1.6 & - & & \\
\hline & & average. & $\max$. & $\min$. & \\
\hline & ngth & 5.04 & 5.25 & 4.7 & \\
\hline & idth & 3.01 & 3.20 & 2.7 & \\
\hline
\end{tabular}

d) Percentage of Shinziromai-- $-66.88 \%$

e) Depth of longitudinal furrow. $(\mu)$

$\begin{array}{rrr}\text { average. } & \max . & \min . \\ 43.47 & 77.85 & 17.30\end{array}$

f) Specific gravity. -1.443 .

g) Saccharifying quality.-17.67\%

h) Hardness. (kilo)

\begin{tabular}{|c|c|c|}
\hline under 3.75 & & 20 grains. \\
\hline $3.75-5.63$ & & $30 \prime \prime$ \\
\hline $5.63-7.50$ & & - \\
\hline $7.50-9.38$ & & - \\
\hline above 9.38 & & - \\
\hline verage. & $\max$ & min. \\
\hline 4.17 & 5.59 & 2.51 \\
\hline
\end{tabular}

i) Volume of $100 \mathrm{~g} .-119.0 \mathrm{c} . \mathrm{c}$.

j) Weight of 1,000 grains.- $-22.34 \mathrm{~g}$.

k) Volume of 1,000 grains.-15.4c.c.

l) Phytin. $-0.09 \%$

n) Absorption of water.

time steeped.

$$
2 \mathrm{~h} \text {. }
$$

$\%$ absorbed.

21.26

p) Chemical composition.-

Water
D. R. sugar
Starch
Crude fat
" fibre
" protein
Ash

13.40

0.51

77.23

0.36

0.28

6.35

0.54

Phosphoric acid

0.21 unhydrous.

$$
\begin{array}{r}
0.59 \\
90.58 \\
0.42 \\
0.32 \\
7.45 \\
0.63 \\
0.25
\end{array}
$$


L) Production of Yelime prefecture.

a) Percentage of full grown grains.- $-92.93 \%$

b) External appearance and luster.-White lustrous, of middle size.

c) Size of grains. (m.m.)

\begin{tabular}{|c|c|c|c|c|c|}
\hline thickness & above & 2.2 & $34.39 \mathrm{~g}$. & 17 & grains. \\
\hline$"$ & "I & 2.0 & $44.88 \quad \prime$ & 23 & $\prime \prime$ \\
\hline " & " & 1.8 & $20.18 \prime \prime$ & 10 & $\prime \prime$ \\
\hline$"$ & $" \prime$ & 1.6 & $0.38 \prime \prime$ & - & \\
\hline$\prime \prime$ & under & 1.6 & $0.02 "$ & - & \\
\hline & ngth & $\begin{array}{c}\text { average. } \\
5.21\end{array}$ & $\begin{array}{r}\max . \\
5.65\end{array}$ & $\begin{array}{r}\min . \\
4.50\end{array}$ & \\
\hline & idth & 3.11 & 3.20 & 2.60 & \\
\hline
\end{tabular}

d) Percentage of Shinziromai._-69.68\%

e) Depth of longitudinal furrow. $(\mu)$

$\begin{array}{ccc}\text { average. } & \max . & \min . \\ 36.76 & 77.85 & 8.65\end{array}$

f) Specific gravity. 1.439

g) Saccharifying quality.- $17.94 \%$

h) Hardness. (kilo)

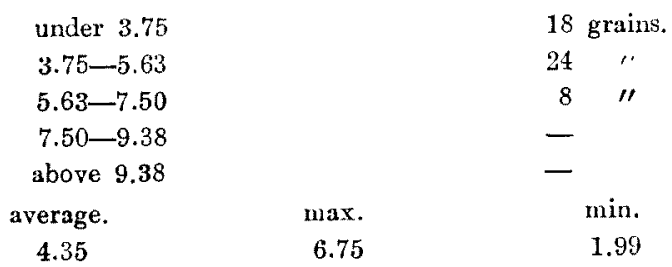

i) Volume of $100 \mathrm{~g} .-123.0$ c.c.

j) Weight of 1,000 grains. $-23.44 \mathrm{~g}$.

k) Volume of 1,000 grains.-16.3c.c.

l) Phytin. - $0.01 \%$

n) Absorption of water.

time steeped.

$$
2 \mathrm{~h} .
$$

$\%$ absorbed.

20.44

p) Chemical composition :-

$\begin{array}{lrr}\text { Water } & 13.27 & - \\ \text { D. R. sugar } & 0.24 & 0.28 \\ \text { Starch } & 77.47 & 90.17 \\ \text { Crude fat } & 0.22 & 0.26 \\ \quad \text { " fibre } & 0.36 & 0.41 \\ \quad \text { " protein } & 7.20 & 8.38 \\ \text { Ash } & 0.42 & 0.49 \\ \text { Phosphoric acid } & 0.16 & 0.19\end{array}$


M) Production of Fulaslima prefecture.

a) Percentage of full grown grains.

$-92.5 \%$

b) External appearance and luster.— Nearly same as J).

c) Size of grains. (m.m.)

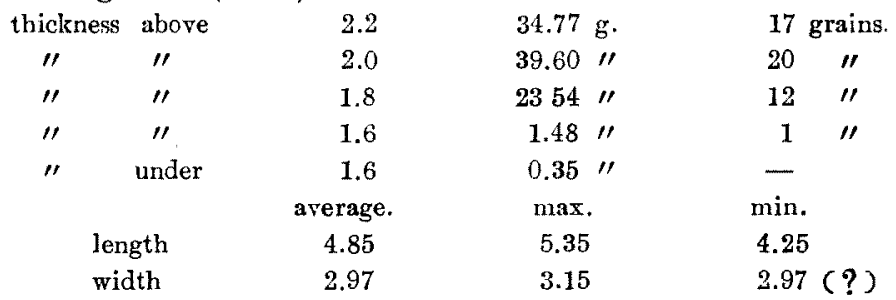

d) Percentage of Shinziromai- $43.08 \%$

e) Depth of longitudinal furrow. ( $\mu)$

$\begin{array}{ccc}\text { average. } & \max . & \operatorname{mim} . \\ 41.52 & 86.50 & 17.30\end{array}$

f) Specific gravity.- -1.443

g) Saccharifying quality.-32.75\%

h) Hardness. (kilo)

$\begin{array}{ccc}\text { under } 3.75 & & 12 \text { grains. } \\ 3.75-5.63 & & 36 " \prime \\ 5.63-7.50 & & 2 \quad " \\ 7.50-9.38 & & - \\ \text { above } 9.38 & & - \\ \text { average. } & \max . & \min . \\ 4.17 & 6.19 & 2.18\end{array}$

i) Volume of $100 \mathrm{~g}$. 117.0c.c.

j) Weight of 1,000 grains. $-21.62 \mathrm{~g}$.

23.55g. (Shinziromai)

k) Volume of 1,000 grains. 15.2c.c.

1) Phytin. $-0.03 \%$

16.4c.c. (Shinziromai)

n) Absorption of water. time steeped.

$2 \mathrm{~h}$.

$\%$ absorbed.

18.4

p) Chemical composition.-

$\begin{array}{lrr}\text { Water } & 14.04 & - \\ \text { D. R. sugar } & 0.34 & 0.40 \\ \text { Starch } & 77.53 & 90.60 \\ \text { Crude fat } & 0.36 & 0.42 \\ \quad \text { " fibre } & 0.20 & 0.23 \\ \quad \text { " protein } & 6.78 & 7.92 \\ \text { Ash } & 0.37 & 0.43 \\ \text { Phosphoric acid } & 0.18 & 0.22\end{array}$


N) Production of Fukushima prefecture.
a) Percentage of full grown grains.
$84.11 \%$
b) External appearance and luster.-Nearly same as M).
c) Size of grains. (m.m.)

\begin{tabular}{|c|c|c|c|c|c|}
\hline thickness & above & 2.2 & $38.04 \mathrm{~g}$. & $19 \xi$ & rains. \\
\hline " & $" \prime$ & 2.0 & $25.10 "$ & 12 & $\prime \prime$ \\
\hline " & 11 & 1.8 & $31.58 \prime \prime$ & 16 & $\prime \prime$ \\
\hline " & $\prime \prime$ & 1.6 & $3.86 " \prime$ & 2 & $" \prime$ \\
\hline " & under & 1.6 & $1.32 \prime \prime$ & 1 & $\prime \prime$ \\
\hline & length & $\begin{array}{c}\text { average. } \\
4.92\end{array}$ & $\begin{array}{l}\max . \\
5.25\end{array}$ & $\begin{array}{r}\min . \\
4.30\end{array}$ & \\
\hline & width & 2.94 & 3.35 & 2.60 & \\
\hline
\end{tabular}

d) Percentage of Shinziromai.-39.16\%

e) Depth of longitudinal furrow. $(\mu)$

$\begin{array}{rrr}\text { average. } & \max . & \min . \\ 43.47 & 77.85 & 17.30\end{array}$

f) Specific gravity. -1.439

g) Saccharifying quality.-30.66\%

h) Hardness. (kilo)

\begin{tabular}{ccc} 
under 3.75 & \multicolumn{2}{c}{22 grains. } \\
$3.75-5.63$ & $26 \quad " \prime$ \\
$5.63-7.50$ & & $2 \quad \prime \prime$ \\
$7.50-9.38$ & & - \\
above 9.38 & & - \\
average. & $\max$ & \\
3.83 & 6.00 & min. \\
& & 2.03
\end{tabular}

i) Volume of $100 \mathrm{~g}$. - $115.0 \mathrm{c} . \mathrm{c}$.

j) Weight of 1,000 grains $-21.7 \mathrm{~g}$.

k) Volume of 1,000 grains.-15.2c.c.

n) Absorption of water.

time steeped.

$2 \mathrm{~h}$.

p) Chemical composition.-

Water

D. R. sugar

Starch

Crude fat

" fibre

/" protein

Ash

Phosphoric acid
14.26

0.23

77.41

0.34

0.35

6.69

0.30

0.18
$\%$ absorbed.

18.72

unhydrous.

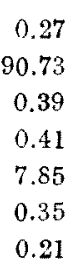


O) Production of Miyagi prefecture.

a) Percentage of full grown grains. $-88.48 \%$

b) External appearance and luster.- Nearly same as before.

c) Size of grains. (m.m.)

\begin{tabular}{|c|c|c|c|c|c|}
\hline \multicolumn{2}{|c|}{ thickness above } & 2.2 & $3.50 \mathrm{~g}$. & \multicolumn{2}{|c|}{2 grains. } \\
\hline " & $\prime \prime$ & 2.0 & $15.94 " \prime$ & 8 & $" \prime$ \\
\hline$" \prime$ & $\prime \prime$ & 1.8 & $74.62 \prime \prime$ & 37 & $" \prime$ \\
\hline$" \prime$ & $\prime \prime$ & 1.6 & $5.19 \prime \prime$ & 3 & $\prime \prime$ \\
\hline$" 1$ & under & 1.6 & $0.82 \quad \prime \prime$ & - & \\
\hline & & average. & $\max$ & $\min$. & \\
\hline & gth & 4.81 & 5.2 & 4.25 & \\
\hline & dth & 2.78 & 3.0 & 2.55 & \\
\hline
\end{tabular}

d) Percentage of Shinziromai.-12.44\%

e) Depth of longitudinal furrow. $(\mu)$
average.
niax.
$\min$.
40.87
69.20
17.30

f) Specific gravity.- 1.439

g) Saccharifying quality. $-26.83 \%$

h) Hardness. (kilo)

$\begin{array}{cccc}\text { under } 3.75 & & 25 \text { grains. } \\ 3.75-5.63 & & 24 & \prime \prime \\ 5.63-7.50 & & 1 & \prime \prime \\ 7.50-9.38 & & - & \\ \text { above } 9.38 & & - & \\ \text { average. } & \max . & & \mathrm{min} . \\ 3.73 & 6.45 & & 2.44\end{array}$

i) Volume of $100 \mathrm{~g} \cdot-119.0$ c.c.

j) Weight of 1,000 grains. $-18.74 \mathrm{~g}$.

k) Volume of 1,000 grains.-13.0c.c.

n) Absorption of water.

time steeped.

$2 \mathrm{~h}$.

$\%$ absorbed.

17.34

p) Chemical composition :-

$\begin{array}{lrr}\text { Water } & 13.58 & - \\ \text { D. R. sugar } & 0.32 & 0.36 \\ \text { Starch } & 78.70 & 90.26 \\ \text { Crude fat } & 0.36 & 0.41 \\ \quad \prime \prime \text { fibre } & 0.50 & 0.57 \\ \quad \prime \prime \text { protein } & 7.04 & 8.07 \\ \text { Ash } & 0.29 & 0.32 \\ \text { Phosphoric acid } & 0.17 & 0.20\end{array}$


P) Production of Niigdta prefecture.

a) Percentage of full grown grains. $-90.56 \%$

b) External appearance and luster.-Slightly brownish white colour, destitute of luster, grains were of middle size and not fat.

c) Size of grains. (m.m.)

\begin{tabular}{|c|c|c|c|c|c|}
\hline \multicolumn{2}{|c|}{ thickness above. } & 2.2 & $22.80 \mathrm{~g}$. & \multicolumn{2}{|c|}{11 grains. } \\
\hline "I & 11 & 20 & $45.28 \prime \prime$ & 23 & $r$ \\
\hline$\prime \prime$ & $\prime \prime$ & 1.8 & $28.75 \prime \prime$ & 14 & $\prime \prime$ \\
\hline$" \prime$ & $" \prime$ & 1.6 & $1.62 \prime \prime$ & 1 & $" \prime$ \\
\hline$\prime \prime$ & under & 1.6 & $1.50 \prime \prime$ & 1 & $\prime \prime$ \\
\hline & ngth & average. & $\begin{array}{r}\max \\
540\end{array}$ & $\min$ & \\
\hline & idth & 2.88 & 3.10 & 2.60 & \\
\hline
\end{tabular}

d) Percentage of Shinziromai._- $14.2 \%$

e) Depth of longitudinal furrow. $(\mu)$

$\begin{array}{rrr}\text { average. } & \max . & \operatorname{min.} \\ 37.8 \mathrm{t} & 51.90 & 17.30\end{array}$

f) Specific gravity.- 1.449

g) Saccharifying quality.-32.85\%

h) Hardness. (kilo)

\begin{tabular}{|c|c|c|}
\hline under 3.75 & & 4 grains \\
\hline $3.75-5.63$ & & 26 \\
\hline $5.63-7.50$ & & 20 \\
\hline $7.50-9.38$ & & - \\
\hline above 9.38 & & - \\
\hline average. & $\max$. & $\min$. \\
\hline 5.19 & 6.92 & \\
\hline
\end{tabular}

i) Volume of $100 \mathrm{~g} . \longrightarrow 121.5 \mathrm{c} . \mathrm{c}$.

j) Weight of 1,000 grains. $-21.73 \mathrm{~g}$.

k) Volume of 1,000 grains.--15.8c.c.

l) Phytin. $-0.08 \%$

n) Absorption of water.

time steeped.

$2 \mathrm{~h}$.

$\%$ absorbed.

19.4

p) Chemical composition :-

$\begin{array}{lr}\text { Water } & 13.35 \\ \text { D. R. sugar } & 0.32 \\ \text { Starch } & 77.04 \\ \text { Crude fat } & 0.30 \\ \quad \text { " fibre } & 0.51 \\ \quad \text { " protein } & 7.46 \\ \text { Ash } & 0.33 \\ \text { Phosphoric acid } & 0.18\end{array}$

unhydrous.

-
0.37
89.56
0.42
0.59
8.66
0.38
0.21


Q) Production of Aichi prefecture.

a) Percentage of full grown grains. $-81.08 \%$

b) External appearance and luster--Nearly same as before.

c) Size of grains. (m.m.)

\begin{tabular}{|c|c|c|c|c|c|}
\hline thick & above & 2.2 & $30.05 \mathrm{~g}$ & 15 & cains. \\
\hline$\prime \prime$ & 11 & 2.0 & $36.00 \prime \prime \prime$ & 18 & $\prime \prime$ \\
\hline$\prime \prime$ & " & 1.8 & $27.88 \prime \prime$ & 14 & $\prime \prime$ \\
\hline$\prime \prime$ & $\prime \prime$ & 1.6 & $3.93 \prime$ & 2 & " \\
\hline$\prime \prime$ & under & 1.6 & $2.10 " \prime$ & 1 & $\prime \prime$ \\
\hline & gth & $\begin{array}{c}\text { average. } \\
4.97\end{array}$ & $\begin{array}{r}\max . \\
5.30\end{array}$ & $\begin{array}{r}\min . \\
4,55\end{array}$ & \\
\hline & & 2.93 & 3.10 & 2.65 & \\
\hline
\end{tabular}

d) Percentage of Shinziromai.-57.14\%

e) Depth of longitudinal furrow. $(\mu)$

$\begin{array}{rrr}\text { average. } & \max . & \min . \\ 36.76 & 69.20 & 17.30\end{array}$

f) Specific gravity.-1.439

g) Saccharifying quality.-18.85\%

h) Hardness. (kilo)

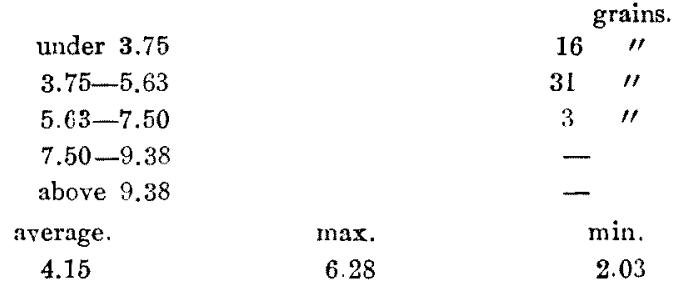

i) Volume of $100 \mathrm{~g} \cdot-126.0$ c.c.

j) Weight of 1,000 grains. $-20.85 \mathrm{~g}$.

k) Volume of 1,000 grains.-14.60c.c.

n) Absorption of water.

time steeped.

$2 \mathrm{~h}$.

p) Chemical composition :-

Water
D. R. sugar
Starch
Crude fat
$\quad$ " fibre
$\quad$ " protein
Ash
Phosphoric acid

$$
\begin{aligned}
& 19.96 \\
& \text { absorbed. }
\end{aligned}
$$

unhydrous.

$$
\begin{array}{r}
0.38 \\
90.05 \\
0.44 \\
0.73 \\
7.88 \\
0.53 \\
0.25
\end{array}
$$


R) Production of Diye prefecture.

a) Percentage of full grown grains.-67.31\%

b) External appearance and luster.—White glassy luster, grains were not fat and of middle size.

c) Size of grains. (m.m.)

\begin{tabular}{|c|c|c|c|c|c|}
\hline \multicolumn{2}{|c|}{ thickness above } & 3.2 & $6.89 \mathrm{~g}$. & \multicolumn{2}{|c|}{3 grains. } \\
\hline 11 & $\prime \prime$ & 2.0 & $33.94 "$ & 17 & $\prime \prime$ \\
\hline " & $\prime \prime$ & 1.8 & $55.21 / \prime$ & 28 & $\prime \prime$ \\
\hline$\prime \prime$ & $\prime \prime$ & 1.6 & $3.51 \prime \prime$ & 2 & 11 \\
\hline "I & under & 1.6 & $0.35 \prime \prime$ & - & \\
\hline & ngth & $\begin{array}{c}\text { average. } \\
5.00\end{array}$ & $\begin{array}{r}\max \\
5.40\end{array}$ & $\begin{array}{r}\min . \\
4.80\end{array}$ & \\
\hline & didth & 2.90 & 3.00 & 260 & \\
\hline
\end{tabular}

d) Percentage of Shinziromai.- $48.0 \%$

e) Depth of longitudinal furrow. $(\mu)$
average.
max.
$\operatorname{min.}$
39.79
77.85
25.95

f) Specific gravity.-1.439

g) Saccharifying quality.—-24.71\%

h) Hardness. (kilo)

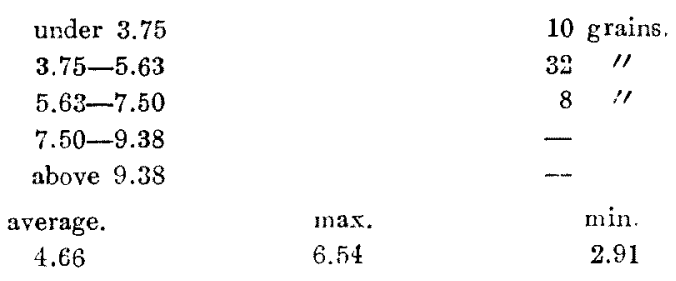

i) Volume of $100 \mathrm{~g}$ - - $122.0 \mathrm{c} . \mathrm{c}$.

j) Weight of 1,000 grains.- $-20.85 \mathrm{~g}$.

k) Volume of 1,000 grains. $-14.6 \mathrm{c} \mathrm{c}$.

n) Absorption of water.

time steeped.

$$
2 \mathrm{~h} .
$$

$\%$ absorbed.

20.30

p) Chemical composition :-

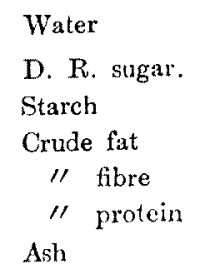

Phosphoric acid
12.99

0.46

78.49

0.25

0.52

6.61

0.47

0.18 unhydrous.

$$
\begin{array}{r}
0.53 \\
90.43 \\
0.29 \\
0.59 \\
7.61 \\
0.54 \\
0.21
\end{array}
$$


S) Production of Okayame prefecture.

a) Percentage of full grown grains.-96.8\%

b) External appearance and luster.-Same as A)

c) Size of grains. (m.m.)

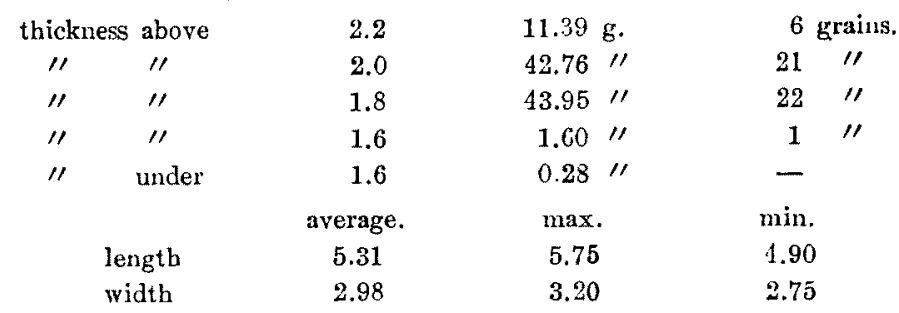

d) Percentage of Shinziromai.- $55.3 \%$

e) Depth of longitudinal furrow. $(\alpha)$

$\begin{array}{ccc}\text { average. } & \max . & \min . \\ 11.63 & 34.60 & 0\end{array}$

f) Specific gravity. -1.439

g) Saccharifying quality._-29.53\%

h) Hardness. (kilo)

\begin{tabular}{|c|c|c|}
\hline urider 3.75 & & 31 grains \\
\hline $3.75-5.63$ & & $19 \quad / 7$ \\
\hline $5.63-7.50$ & & - \\
\hline $7.50-9.38$ & & - \\
\hline above 9.38 & & - \\
\hline verage. & $\max$. & min. \\
\hline
\end{tabular}

i) Volume of $100 \mathrm{~g} \cdot-120.0 \mathrm{c} . \mathrm{c}$.

j) Weight of 1,000 grains. $-23.01 \mathrm{~g}$.

k) Volume of 1,000 grains. 16.0 c.c.

1) Phytin.- $0.01 \%$

n) Absorption of water.

time stceped.

$2 \mathrm{~h}$.

$\%$ absorbed.

21.2

p) Chemical composition :-

$\begin{array}{lrr}\text { Water } & 12.75 & - \\ \text { D. R. sugar } & 0.45 & 0.52 \\ \text { Starch } & 78.53 & 90.93 \\ \text { Crude fat } & 0.21 & 0.24 \\ \quad \text { " fibre } & 0.52 & 0.60 \\ \text { "I protein } & 6.35 & 7.35 \\ \text { Ash } & 0.32 & 0.36 \\ \text { Phosphoric acid } & 0.15 & 0.18\end{array}$


T) Production of Hiroshima prefecture.

a) Percentage of full grown grains.-87.91\%

b) External appearance and luster.—Nearly same as before.

c) Size of grains. (m.m.)

\begin{tabular}{|c|c|c|c|c|c|c|}
\hline \multicolumn{2}{|c|}{ thickness above } & 2.2 & 14.11 & & \multicolumn{2}{|c|}{7 grains. } \\
\hline "I & 11 & 20 & 37.05 & "I & 19 & 11 \\
\hline$" \prime$ & $\prime \prime$ & 1.8 & 41.37 & $\prime \prime$ & 21 & " \\
\hline$" \prime$ & 11 & 1.6 & 4.99 & $\prime \prime$ & 2 & 11 \\
\hline 11 & under & 1.6 & 2.40 & $\prime \prime$ & 1 & 11 \\
\hline & gth & $\begin{array}{c}\text { average. } \\
4.97\end{array}$ & $\begin{array}{r}\max . \\
5.40\end{array}$ & & $\begin{array}{r}\min . \\
4.60\end{array}$ & \\
\hline & dth & 2.86 & 3.00 & & 2.65 & \\
\hline
\end{tabular}

d) Percentage of Shinziromai, $-56.88 \%$

e) Depth of longitudinal furrow. $(\mu)$

$\begin{array}{rrr}\text { average. } & \max . & \text { min. } \\ 21.02 & 43.25 & 5.19\end{array}$

f) Specific gravity.- 1.439

g) Saccharifying quality.- $-32.50 \%$

h) Hardness. (kilo)

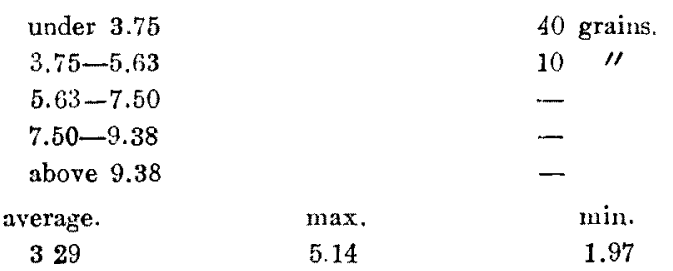

i) Volume of $100 \mathrm{~g}$. 118.0c.c.

j) Weight of 1,000 grains. $-21.18 \mathrm{~g}$.

k) Volume of 1,000 grains.-14.7c.c.

1) Phytin. 0.01

n) Absorption of water.

time steeped.

$2 \mathrm{~h}$.

$\%$ absorbed.

20.26

p) Chemical composition :-

$\begin{array}{lrr}\text { Water } & 13.04 & - \\ \text { D. R. rugar } & 0.47 & 0.54 \\ \text { Starch } & 78.86 & 90.87 \\ \text { Crude fat } & 0.20 & 0.23 \\ \quad \text { " fibre } & 0.31 & 0.35 \\ \quad \text { " protein } & 6.61 & 7.61 \\ \text { Ash } & 0.3 \pm & 0.39 \\ \text { Phosphoric acid } & 0.15 & 0.17\end{array}$




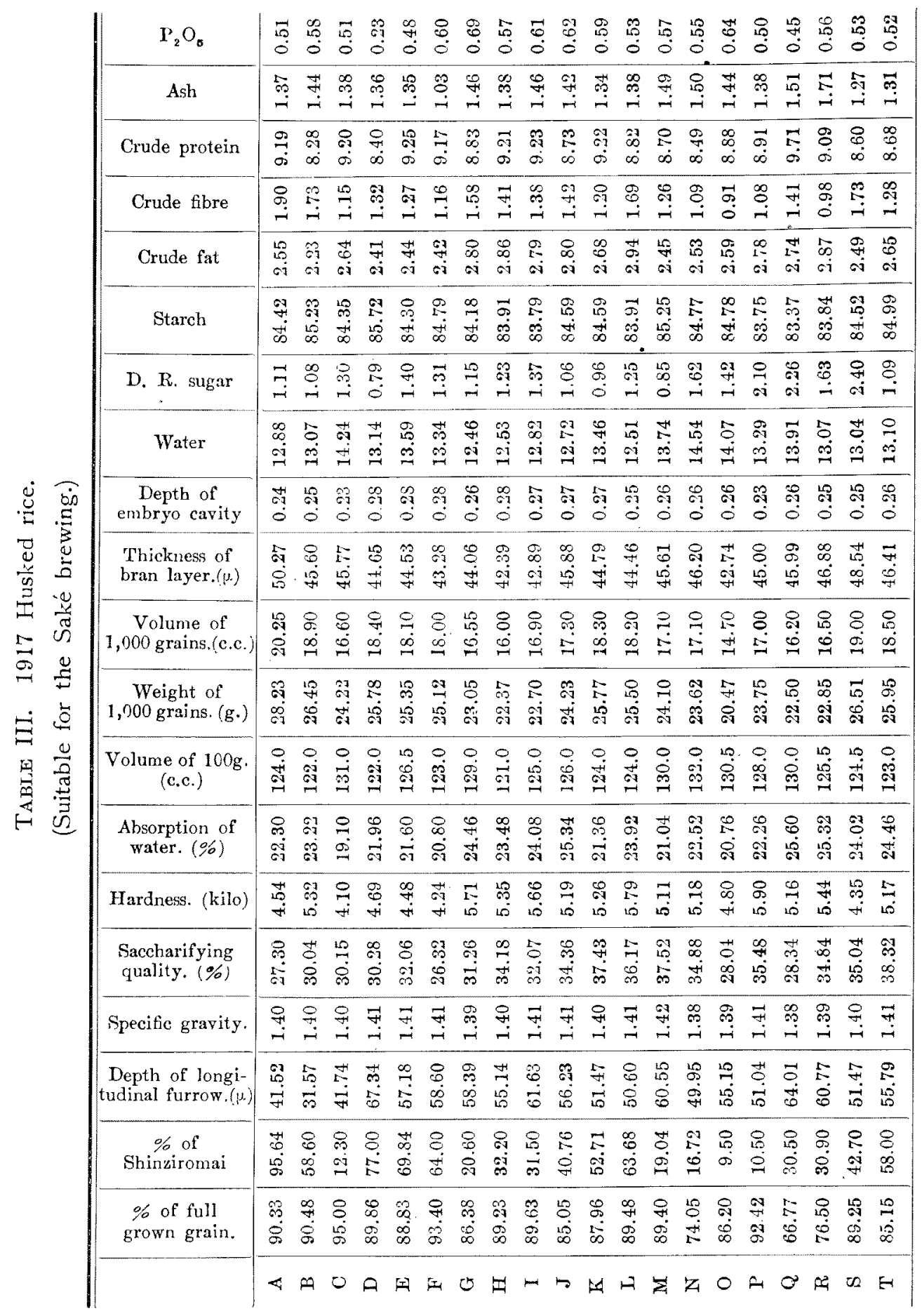




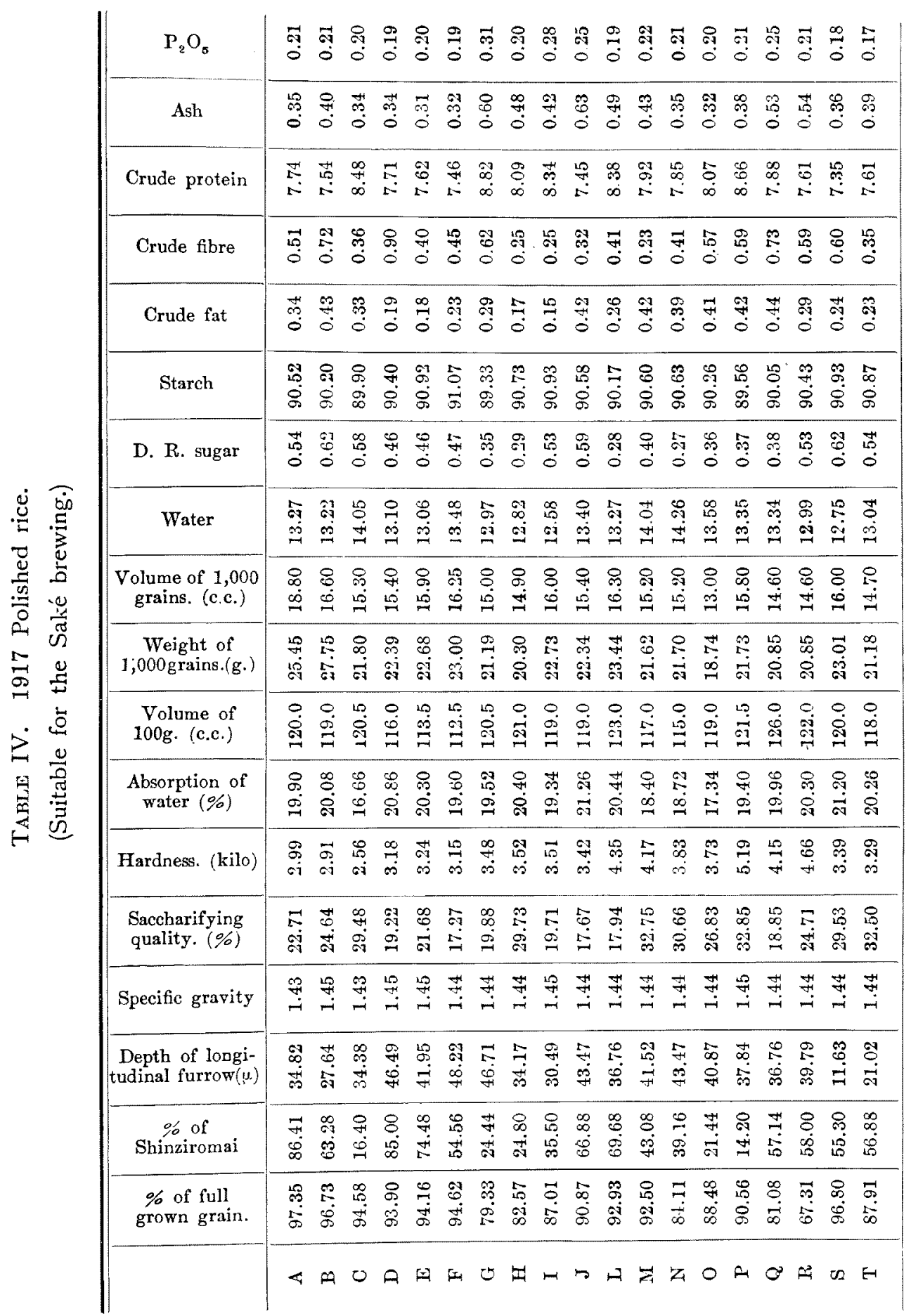


TABre V. 1917 Husked rice.

\begin{tabular}{|c|c|c|c|}
\hline & $\begin{array}{c}\text { General average } \\
\text { (20 var.) }\end{array}$ & $\begin{array}{l}\text { Average of those } \\
\text { under general } \\
\text { average. }\end{array}$ & $\begin{array}{c}\text { Average of those } \\
\text { above general } \\
\text { average. }\end{array}$ \\
\hline Percentage of full grown grains. $\ldots$ & 86.77 & $90.41^{(13)}$ & $80.02(7)$ \\
\hline Percentage of shinziromai. ............ & 42.03 & $23.25(11)$ & $65.05^{(9)}$ \\
\hline Depth of longitudinal furrow. $(\mu) \ldots$ & 54.01 & $46.17^{(8)}$ & $59.24(12)$ \\
\hline Specific gravity $\ldots \ldots \ldots \ldots \ldots \ldots \ldots \ldots$ & 1.40 & $1.39(10)$ & $1.41^{(1))}$ \\
\hline Saccharifying quality. $(\%)$............ & 32.70 & $29.58^{(10)}$ & $35.82^{(10)}$ \\
\hline Hardness. (kilo) …................... & 5.05 & $4.46^{(7)}$ & $5.51^{(13)}$ \\
\hline Absorption of water. $(\%) \ldots \ldots \ldots \ldots \ldots$ & 22.88 & $21.37(10)$ & $24.39^{(10)}$ \\
\hline Volume of $100 \mathrm{~g}$. (c.c.) $\ldots \ldots \ldots \ldots \ldots \ldots$ & 126.0 & $123.7^{(12)}$ & $129.7(8)$ \\
\hline Weight of 1,000 grains. (g.) $\cdots \ldots \ldots$ & 24.47 & $23.20(11)$ & $26.07^{(9)}$ \\
\hline Volume of 1,000 grains. (c.c.) $\ldots$... & 17.48 & $16.54(11)$ & $18.23^{(9)}$ \\
\hline Thickness of bran layer. ( $\mu$ ) $\ldots \ldots \ldots$ & 45.40 & $43.88^{(10)}$ & $46.72(10)$ \\
\hline Depth of embryo cavity. ................. & 0.26 & $0.25(10)$ & $0.27^{(10)}$ \\
\hline 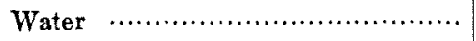 & 13.28 & $12.85(11)$ & $13.79^{(9)}$ \\
\hline D. R. sugar $\ldots \ldots \ldots \ldots \ldots \ldots \ldots \ldots \ldots \ldots \ldots \ldots \ldots$ & 1.32 & $1.10^{(12)}$ & $1.65^{(8)}$ \\
\hline 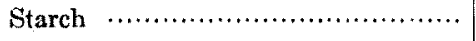 & 84.66 & $84.04(13)$ & $85.04(7)$ \\
\hline Crude fat $\ldots \ldots \ldots \ldots \ldots \ldots \ldots \ldots \ldots \ldots \ldots \ldots \ldots \ldots$ & 2.76 & $2.52(13)$ & $2.83(7)$ \\
\hline Crude fibre $\ldots \ldots \ldots \ldots \ldots \ldots \ldots \ldots \ldots$ & 1.35 & $1.15(11)$ & $1.58^{(9)}$ \\
\hline Crude protein $\ldots \ldots \ldots \ldots \ldots \ldots \ldots \ldots \ldots \ldots \ldots$ & 8.92 & $8.66(11)$ & $9.25^{(9)}$ \\
\hline 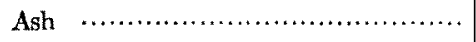 & 1.40 & $132(11)$ & $1.49^{(0)}$ \\
\hline Phosphoric acid $\ldots \ldots \ldots \ldots \ldots \ldots \ldots \ldots \ldots \ldots \ldots$ & 0.52 & $0.45^{(0)}$ & $6.58(14)$ \\
\hline
\end{tabular}

Table VI. 1917 Polished rice.

\begin{tabular}{|c|c|c|c|}
\hline ' & $\begin{array}{l}\text { General average } \\
\left(\begin{array}{l}20 \text { var })\end{array}\right.\end{array}$ & $\begin{array}{l}\text { Average of those } \\
\text { under general } \\
\text { average. }\end{array}$ & $\begin{array}{c}\text { Average of those } \\
\text { above general } \\
\text { average. }\end{array}$ \\
\hline$\%$ of full grown grains. $\ldots \ldots \ldots \ldots \ldots$ & 87.25 & $93.69: 13)$ & $76.24(7)$ \\
\hline$\%$ of Shinziromai $\ldots \ldots \ldots \ldots \ldots \ldots \ldots \ldots$ & 49.68 & $31.01(9)$ & $65.97(11)$ \\
\hline Depth of longitudinal furrow. (p.)... & 37.56 & $29.75(9)$ & $43.96(11)$ \\
\hline 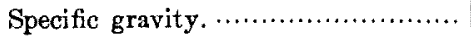 & 1.44 & $1.44^{(12)}$ & $1.45(8)$ \\
\hline Sacch. quality. $(\%) \quad \ldots \ldots \ldots \ldots \ldots \ldots$ & 24.17 & $18.98^{(10)}$ & $29.37(10)$ \\
\hline Hardness. (kilo), $\ldots \ldots \ldots \ldots \ldots \ldots \ldots \ldots \ldots$ & 3.62 & $3.20(11)$ & $4.22^{(0)}$ \\
\hline Absorption of water. (\%) $\% \ldots \ldots \ldots$ & 19.61 & $18.58(0)$ & $20.44(11)$ \\
\hline Volume of $100 \mathrm{~g}$. (c.c.) $\ldots \ldots \ldots \ldots \ldots$ & 119.30 & $116.8(10)$ & $121.8(10)$ \\
\hline Weight of 1,000 grains. (g.) $\cdots \cdots \cdots$ & 22.61 & $21.22(12)$ & $23.43^{(8)}$ \\
\hline Volume of 1,000 grains. (c.c.) $\ldots \ldots$ & 15.55 & $14.84^{(11)}$ & $16.42^{(9)}$ \\
\hline 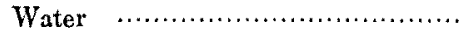 & 13.34 & $13.01(11)$ & $13.74^{(0)}$ \\
\hline D. R. sugar $\ldots \ldots \ldots \ldots \ldots \ldots \ldots \ldots \ldots \ldots \ldots \ldots \ldots \ldots \ldots$ & 0.45 & $0.34^{(9)}$ & $0.53(11)$ \\
\hline 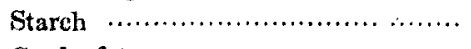 & 90.42 & $90.00(0)$ & $90.75^{(11)}$ \\
\hline Crude fat $\ldots \ldots \ldots \ldots \ldots \ldots \ldots \ldots \ldots \ldots \ldots \ldots \ldots \ldots \ldots \ldots$ & 0.30 & $0.22(11)$ & $0.40(9)$ \\
\hline Crude protein $\ldots \ldots \ldots \ldots \ldots \ldots \ldots \ldots \ldots \ldots$ & 7.94 & $7.64^{(12)}$ & $8.39^{(8)}$ \\
\hline Crude fibre $\ldots \ldots \ldots \ldots \ldots \ldots \ldots \ldots, \ldots, \ldots$ & 0.49 & $0.34(10)$ & $0.64(10)$ \\
\hline 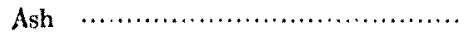 & 0.43 & $0.36(12)$ & $0.53(s)$ \\
\hline 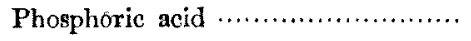 & 0.21 & $0.20(14)$ & $0.25^{(\theta)}$ \\
\hline
\end{tabular}




\section{Summary of the 1917 production.}

Percentage of Shinziromai, specific gravity, saccharifying quality, absorption of water, weight of 1,000 grains, starch, crude fat, crude fibre, crude protein and phosphoric acid were somewhat higher than those of the production of 1916 suited for Saké brewing, while the percentage of full grown grains, depth of longitudinal furrow, hardness, volume of $100 \mathrm{~g}$., thickness of bran layer, depth of embryo cavity, water, sugar and ash were lower when compared with those of 1916 suited for Saké brewing.

\section{Production.}

a) Husked rice, suitable for the Saké brewing. 21 (A-U) varieties.

A) Production of Okayama prefecture.

a) Percentage of full grown grains.- $91.2 \%$

b) External appearance and luster.-- Straw yellow waxy luster, grains were fat and large.

c) Size of grains (m.m.)

thickness above
"
"
"
"

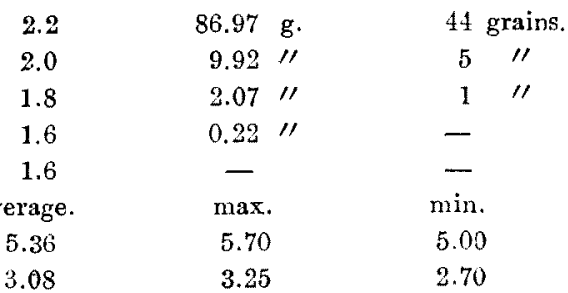

d) Percentage of Shinziromai._- $70.40 \%$

e) Depth of longitudinal furrow. $(\mu)$

$\begin{array}{rrr}\text { average. } & \max . & \min . \\ 35.81 & 51.90 & 17.30\end{array}$

f) Specific gravity. -1.399

h) Hardness. (kilo)

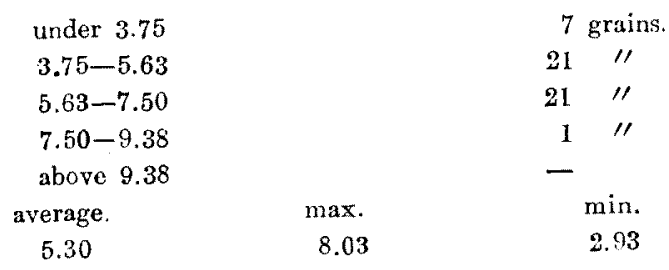

i) Volume of $100 \mathrm{~g} .-121.5 \mathrm{c} . \mathrm{c}$.

j) Weight of 1,000 grains. $-27.80 \mathrm{~g}$.

k) Volume of 1,000 grains.-19.70c.c.

$\mathrm{m})$ Thickness of bran layer. $(\mu)$ 

average.
43.59
$\max$.
49.05
$\min$.
40.05

n) Absorption of water.

time steeped.

$24 \mathrm{~h}$.

$\%$ absorbed.

22.0

p) Chemical composition :-

$\begin{array}{lrr}\text { Water } & 12.96 & - \\ \text { D. R. sugar } & 0.75 & 0.86 \\ \text { Starch } & 74.69 & 85.89 \\ \text { Crude fat } & 7.48 & 2.86 \\ \quad \text { "I protein } & 7.85 & 9.02 \\ \text { Ash } & 1.30 & 1.50\end{array}$

B) Production of Okayama prefecture.

a) Percentage of full grown grains.- $87.5 \%$

b) External appearance and luster.-Nearly same as A).

c) Size of grains. (m.m.)

\begin{tabular}{|c|c|c|c|c|c|}
\hline \multicolumn{2}{|c|}{ thickness above } & 2.2 & $85.06 \mathrm{~g}$ & \multicolumn{2}{|c|}{43 grains. } \\
\hline 11 & "I & 2.0 & $12.38 \prime \prime$ & 6 & $\prime \prime$ \\
\hline " & "1 & 1.8 & $2.32 \prime$ & 1 & 11 \\
\hline "1 & " & 1.6 & $0.18 \prime$ & - & \\
\hline "' & under & 1.6 & $0.07 \prime$ & - & \\
\hline & length & $\begin{array}{c}\text { average. } \\
5.32\end{array}$ & $\begin{array}{r}\max . \\
5.65\end{array}$ & $\begin{array}{r}\text { min. } \\
5.00\end{array}$ & \\
\hline & width & 3.00 & 3.20 & 2.80 & \\
\hline
\end{tabular}

d) Percentage of Shinziromai.— $75.34 \%$

e) Depth of longitudinal furrow. $(\mu)$

$\begin{array}{ccc}\text { average. } & \text { max. } & \min . \\ 40.40 & 60.55 & \mathbf{2 0 . 7 6}\end{array}$

f) Specific gravity.-1.403

h) Hardness. (kilo)

under 3.75

$3.75-5.63$

$5.63-7.50$

$7.50-9.38$

above 9.38

average.

4.97

$\max$.

6.81

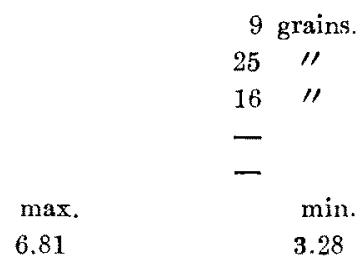

i) Volume of $100 \mathrm{~g} \cdot-121.0 \mathrm{c} . \mathrm{c}$.

j) Weight of 1,000 grains. $-27.20 \mathrm{~g}$.

k) Volume of 1,000 grains.-19.25c.c.

m) Thickness of bran layer. $(\mu)$
average.
44.48
$\max$.
47.93
$\min$.
39.15 
n) Absorption of water.

p) Chemical composition :-

time steeped.

$24 \mathrm{~h}$.

$\%$ absorbed.

$21.6 t$

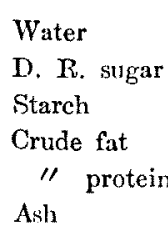

13.26

0.85

75.09

2.22

7.30

1.30 unhydrous.

$$
\begin{array}{r}
0.98 \\
86.35 \\
2.55 \\
8.40 \\
1.50
\end{array}
$$

C) Production of Okayama prefecture.

a) Percentage of full grown grains._- $83.5 \%$

b) External appearance and luster.— Nearly same as B).

c) Size of grains. (m.m.)

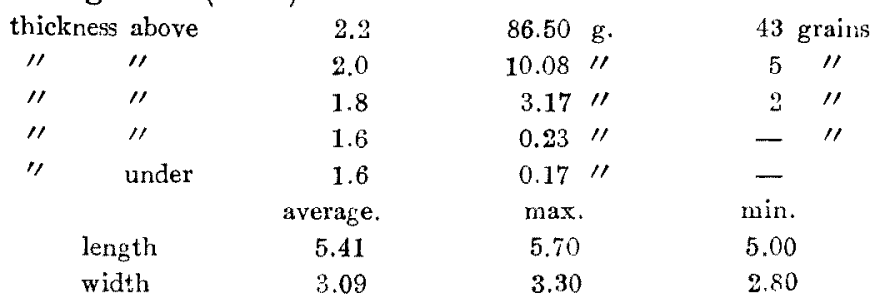

d) Percentage of Shinziromai.-79.20\%

\begin{tabular}{|c|c|c|}
\hline under 3.75 & & 13 grains. \\
\hline $3.75-5.63$ & & $33 \quad / 1$ \\
\hline $5.63-7.50$ & & $\prime \prime$ \\
\hline $750-938$ & & - \\
\hline above 9.38 & & - \\
\hline $\begin{array}{c}\text { verage. } \\
4.31\end{array}$ & $\begin{array}{l}\max . \\
5.78\end{array}$ & $\begin{array}{l}\text { min. } \\
2.50\end{array}$ \\
\hline
\end{tabular}

e) Depth of longitudinal furrow. $(\mu)$

$\begin{array}{rcc}\text { average. } & \max . & \min . \\ 38.62 & 57.09 & 20.76\end{array}$

f) Specific gravity.- 1.399

h) Hardness. (kilo)

i) Volume of $100 \mathrm{~g} .124 .5 \mathrm{c} . \mathrm{c}$.

j) Weight of 1,000 grains. - $-27.85 \mathrm{~g}$.

k) Volume of 1,000 grains.-19.85c.c.

m) Thickuess of bran layer. $(\mu)$

$$
\begin{array}{ccc}
\text { average. } & \text { max. } & \text { min. } \\
44.15 & 47.03 & 39.60
\end{array}
$$

n) Absorption of water.

time steeped.

$2+\mathrm{h}$. 
p) Chemical composition :-

$\begin{array}{lrc} & & \text { unhydrous } \\ \text { Water } & 12.75 & - \\ \text { D. R. sugar } & 1.50 & 1.73 \\ \text { Starch } & 74.91 & 86.14 \\ \text { Crude fat } & 1.75 & 2.02 \\ \quad \text { ' protein } & 7.86 & 9.04 \\ \text { Ash } & 1.23 & 1.41\end{array}$

D) Production of Okayama prefccture.

a) Percentage of full grown grains.- $-85.7 \%$

b) External appearance and luster.— Nearly same as C).

c) Size of grains. (m.m.)

\begin{tabular}{|c|c|c|c|c|c|c|}
\hline \multicolumn{2}{|c|}{ thickness above } & 2.2 & 85.90 & g. & 43 & rrains \\
\hline "I & $\prime \prime$ & 2.0 & 11.50 & $\prime \prime$ & 6 & " \\
\hline$\prime \prime$ & $\prime \prime$ & 1.8 & 2.20 & $\prime \prime$ & 1 & $\prime \prime$ \\
\hline " & $\prime \prime$ & 1.6 & 0.25 & $\prime \prime$ & - & \\
\hline " & under & 1.6 & 0.05 & $\prime \prime$ & - & \\
\hline & & average. & $\max$ & & $\min$. & \\
\hline & gth & 5.43 & 5.75 & & 4.90 & \\
\hline & & 3.04 & 3.25 & & 2.65 & \\
\hline
\end{tabular}

d) Percentage of Shinziromai.- $78.3 \%$

e) Depth of longitudinal furrow. $(\mu)$

$\begin{array}{ccc}\text { average. } & \max . & \min . \\ 42.5 \mathrm{I} & 65.36 & 20.76\end{array}$

f) Specific gravity.-1.403

h) Hardness. (kilo)

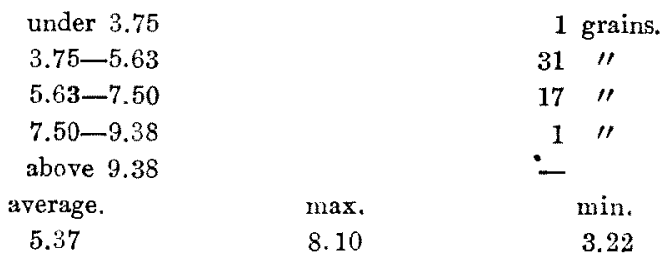

i) Volume of $100 \mathrm{~g} .-121.5 \mathrm{c} . \mathrm{c}$.

j) Weight of 1,000 grains. $-27.55 \mathrm{~g}$.

k) Volume of 1,000 grains.-19.5c.c.

m) Thickness of bran layer. ( $)$

$\begin{array}{ccc}\text { average. } & \max . & \mathrm{min} . \\ 43.22 & 45.90 & 36.68\end{array}$

n) Absorption of water. time steeped. $24 \mathrm{~h}$.

p) Chemical composition :- 


$\begin{array}{lrc} & & \text { unhydrous. } \\ \text { Water } & 12.65 & - \\ \text { D. R. sugar } & 1.05 & \mathbf{1 . 1 9} \\ \text { Starch } & 75.36 & 85.91 \\ \text { Crude fat } & 2.25 & 2.60 \\ \quad \text { " protein } & 7.16 & 8.17 \\ \text { Ash } & 1.45 & \mathbf{1 . 6 5}\end{array}$

E) Korean rice.

a) Percentage of full grown grains.-- $89.28 \%$

b) External appearance and luster.-Straw yellow colour, somewhat poor of luster, grains were not fat and of middle size.

c) Size of grains. (m.m.)

\begin{tabular}{|c|c|c|c|c|c|}
\hline \multicolumn{2}{|c|}{ thickness above } & 2.2 & $21.52 \mathrm{~g}$. & 11 & rains \\
\hline$\prime \prime$ & $\prime \prime$ & 2.0 & $51.10 "$ & 25 & $\prime \prime$ \\
\hline$\prime \prime$ & $\prime \prime$ & 1.8 & $25.45 \prime \prime \prime$ & 13 & $\prime \prime$ \\
\hline$\prime \prime$ & $\prime \prime$ & 1.6 & $1.94 \prime \prime$ & 1 & $\prime \prime$ \\
\hline$\prime \prime$ & under & 1.6 & $0.28 \prime \prime$ & - & \\
\hline & & average. & $\max$ & $\min$. & \\
\hline & gth & 4.97 & 5.30 & 4.60 & \\
\hline & & 2.83 & 3.00 & 2.50 & \\
\hline
\end{tabular}

d) Percentage of Shinziromai.- $56.96 \%$

e) Depth of longitudinal furrow. $(\mu)$

$\begin{array}{ccc}\text { average. } & \max . & \min . \\ 45.97 & 72.66 & 22.49\end{array}$

f) Specific gravity.-1.389

h) Hardness. (kilo)

\begin{tabular}{|c|c|c|}
\hline \multicolumn{2}{|l|}{ under 3.75} & 12 grains. \\
\hline $3.75-5.63$ & & $35 \prime \prime$ \\
\hline $5.63-7.50$ & & $\prime \prime$ \\
\hline $7.50-9.38$ & & - \\
\hline above 9.33 & & - \\
\hline average. & $\max$. & min. \\
\hline 4.24 & 6.75 & 2.40 \\
\hline
\end{tabular}

i) Volume of $100 \mathrm{~g}$. $-126.5 \mathrm{c} . \mathrm{c}$.

j) Weight of 1,000 grains. $-21.55 \mathrm{~g}$.

k) Volume of 1,000 grains.-15.30c.c.

$\mathrm{m})$ Thickness of bran layer. $(\mu)$

$\begin{array}{ccc}\text { average. } & \text { max. } & \text { min. } \\ 41.29 & 49.28 & 37.35\end{array}$

n) Absorption of water.

time steeped.

$24 \mathrm{~h}$.

$\%$ absorbed.

20.24

p) Chemical composition :- 


$\begin{array}{lrc} & & \text { unhydrous. } \\ \text { Water } & 12.77 & - \\ \text { D. R. sugar } & 1.15 & 1.31 \\ \text { Starch } & 74.54 & 84.98 \\ \text { Crude fat } & 2.80 & 3.19 \\ \quad \text { " protein } & 8.00 & 9.12 \\ \text { Ash } & 1.36 & 1.55\end{array}$

F) Korean rice (Koluryomiyako variety).

a) Percentage of full grown grains._- $94.1 \%$

b) External appearance and luster.-Nearly same as A).

c) Size of grains. (m.m.)

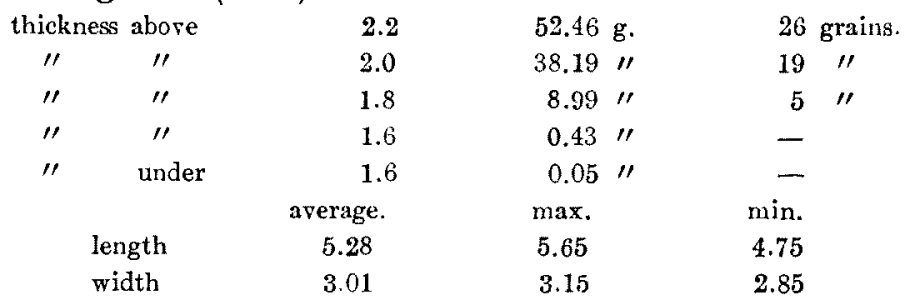

d) Percentage of Shinziromai.— $-87.60 \%$

e) Depth of longitudinal furrow. $(\mu)$

$\begin{array}{ccc}\text { average. } & \max . & \min . \\ 57.05 & 83.04 & 34.60\end{array}$

f) Specific gravity.-1.409

h) Hardness. (kilo)

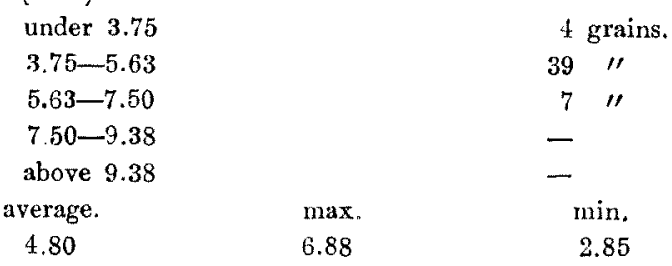

i) Volume of $100 \mathrm{~g} .-122.5$ c.c.

j) Weight of 1,000 grains. $-25.05 \mathrm{~g}$.

k) Volume of 1,000 grains._-17.80c.c,

m) Thickness of bran layer. $(\mu)$

average.

46.78

n) Absorption of water.

time steeped.

$$
24 \mathrm{~h} .
$$

p) Chemical composition :-

Water

13.65

D. R. sugar

0.74 $\max$.

$\min$.

42.53

$\%$ absorbed.

22.2

unhydrous. 


$\begin{array}{lrr}\text { Starch } & 73.44 & 83.79 \\ \text { Crude fat } & 2.32 & 2.69 \\ \text { " protein } & 7.95 & 9.22 \\ \text { Ash } & 1.52 & 1.76 \\ \text { (G-U) omitted } & & \end{array}$

Husked rice unsuitable $(I-X)$ omitted.

\section{Production.}

ß) Polished rice, suitable for the Sake brewing. 21 (A-U) varieties.

A) Production of Okayama prefecture.

a) Percentage of full grown grains.-94.35\%

b) External appearance and luster.- White glassy luster, grains were large and fat.

c) Size of grains. (m.m.)

thickness above
$" \prime$
$\prime \prime$

2.2
2.0
1.8
1.6
1.6
arerage.
5.17
2.96

$\begin{array}{ccc}68.89 \mathrm{~g} . & 35 \text { grains. } \\ 26.55 \prime \prime & 13 \prime \prime \\ 4.45 \prime \prime & 2 \prime \prime \\ 0.32 \prime \prime & - \\ 0.13 \prime \prime & - \\ \max . & \text { min. } \\ 5.50 & 4.70 \\ 3.20 & 2.65\end{array}$

d) Percentage of Shinziromai.- $68.2 \%$

e) Depth of longitudinal furrow. $(\mu)$

$\begin{array}{ccc}\text { average. } & \max . & \min . \\ 24.22 & 43.25 & 17.30\end{array}$

f) Specific gravity.-1.439

h) Hardness. (kilo)

\begin{tabular}{|c|c|c|}
\hline under 3.75 & & 25 grains. \\
\hline $3.75-5.63$ & & $22 \prime \prime$ \\
\hline $5.63-7.50$ & & 3 \\
\hline $7.50-9.38$ & & - \\
\hline above 9.38 & & - \\
\hline $\begin{array}{c}\text { average. } \\
3.81\end{array}$ & $\begin{array}{l}\max . \\
6.15\end{array}$ & $\begin{array}{r}\min . \\
2.34\end{array}$ \\
\hline
\end{tabular}

i) Volume of $100 \mathrm{~g}$. - $114.5 \mathrm{c} . \mathrm{c}$.

j) Weight of 1,000 grains. $-24.90 \mathrm{~g}$.

k) Volume of 1,000 grains.-17.30c.c.

n) Absorption of water. time steeped. $24 \mathrm{~h}$.

p) Chemical composition :- 


$\begin{array}{lrc} & & \text { unhydrous. } \\ \text { Water } & 11.35 & - \\ \text { D. R. sugar } & 0.65 & 0.73 \\ \text { Starch } & 80.17 & 80.59 \\ \text { Crude fat } & 0.97 & 1.09 \\ \quad \text { " protein } & 6.52 & 7.36 \\ \text { Ash } & 0.31 & 0.35\end{array}$

B) Production of Okayama prefecture.

a) Percentage of full grown grains.-94.31\%

b) External appearance and luster._- Nearly same as A).

c) Size of grains. (m.m.)

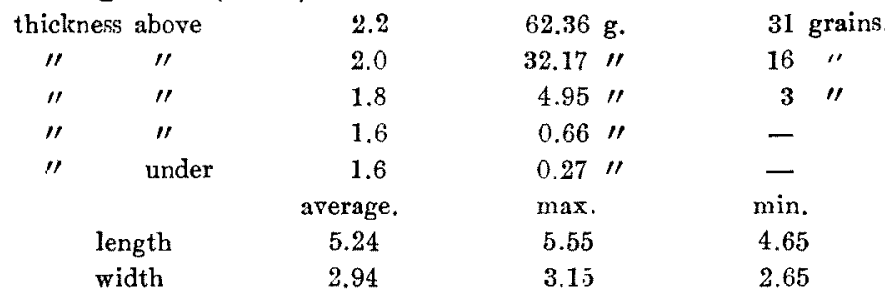

d) Percentage of Shinziromai.- $64.24 \%$

e) Depth of longitudinal furrow. $(\mu)$

$\begin{array}{ccc}\text { average. } & \max . & \min . \\ 35.90 & 57.96 & 38.93\end{array}$

f) Specific gravity.- $-1.429 \%$

h) Hardness. (kilo)

\begin{tabular}{|c|c|c|}
\hline under 3.75 & & 9 grains \\
\hline $3.75-5.63$ & & $26 \quad \prime \prime$ \\
\hline $5.63-7.50$ & & 15 \\
\hline $7.50-9.38$ & & - \\
\hline above 9.38 & & - \\
\hline average. & $\max$. & $\min$. \\
\hline 4.87 & $7.4 \mathrm{I}$ & 2.63 \\
\hline
\end{tabular}

i) Volume of $100 \mathrm{~g}$. $116.5 \mathrm{c.c}$.

j) Weight of 1,000 grains.-25.01g.

k) Volume of 1,000 grains._-17.5c.c.

n) Absorption of water.

time steeped.

$$
2 \mathrm{~h} \text {. }
$$

$\%$ absorbed.

p) Chemical composition :-

22.0

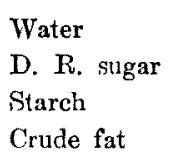

Crude fat

" protein

Ash
14.04

0.65

79.29

0.51

5.49

0.48 unhydrous.

$$
\begin{array}{r}
0.75 \\
91.97 \\
0.59 \\
6.37 \\
0.56
\end{array}
$$


C) Production of Okayama prefecture.

a) Percentage of full grown grains.-91.1

b) External appearance and luster.-Same as A).

c) Size of grains. (m.m.)

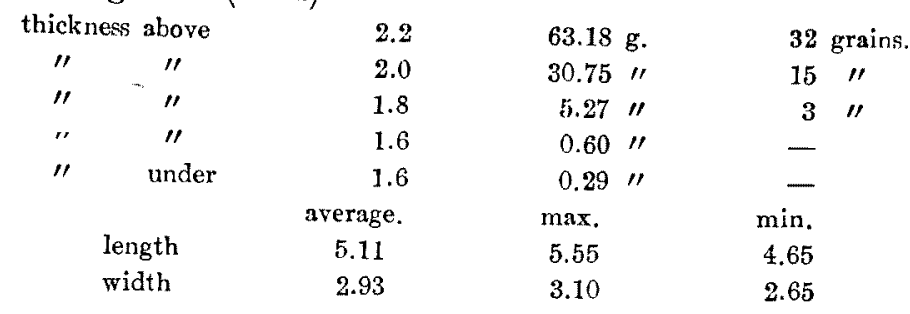

d) Percentage of Shinziromai- -80.60

e) Depth of longitudinal furrow. $(x)$

$\begin{array}{ccc}\text { average. } & \max . & \min . \\ 29.84 & 51.90 & 8.65\end{array}$

f) Specific gravity.- -1.440

h) Hardness. (kilo)

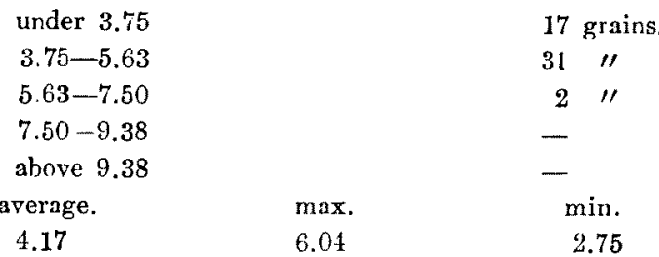

i) Volume of $100 \mathrm{~g} .-116.0$ c.c.

j) Weight of 1,000 grains. $-24.74 \mathrm{~g}$.

k) Volume of 1,000 grains.-17.30c.c.

n) Absorption of water. time steeped.

$2 \mathrm{~h}$.

$\%$ absorbed.

21.4

p) Chemical composition :-

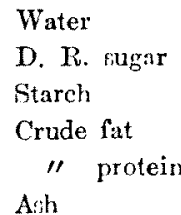

Water

D. R. fugar

tarch

Ash
13.02

1.25

78.98

0.57

5.89

0.36 unhydrous.

1.43

90.82

065

6.77

0.41

D) Production of Okayama prefecture.
a) Percentage of full grown grains.
92.95
b) External appearance and luster.-... Same as A).
c) Size of grains. (m.m.) 


\begin{tabular}{|c|c|c|c|c|c|c|}
\hline \multicolumn{2}{|c|}{ thickness above } & 2.2 & 61.79 & & \multicolumn{2}{|c|}{31 grains. } \\
\hline$\prime \prime$ & $\prime \prime$ & 2.0 & 28.77 & $\prime \prime$ & 14 & $\prime \prime$ \\
\hline$\prime \prime$ & $\prime \prime$ & 1.8 & 8.25 & $\prime \prime$ & 4 & $\prime \prime$ \\
\hline$\prime \prime$ & $\prime \prime$ & 1.6 & 0.94 & $" \prime$ & 1 & $\prime \prime$ \\
\hline$\prime \prime$ & under & 1.6 & 0.30 & $\prime \prime$ & - & \\
\hline & & average. & $\max$ & & $\min$. & \\
\hline & igth & 5.20 & 5.50 & & 4.75 & \\
\hline & $\mathrm{dth}$ & 2.92 & 3.20 & & 2.60 & \\
\hline
\end{tabular}

d) Percentage of Shinziromai. -63.60

e) Depth of longitudinal furrow. $(\mu)$

$$
\begin{array}{ccc}
\text { average. } & \max . & \min . \\
26.81 & 51.90 & 17.30
\end{array}
$$

f) Specific gravity.-1.429

h) Hardness. (kilo)

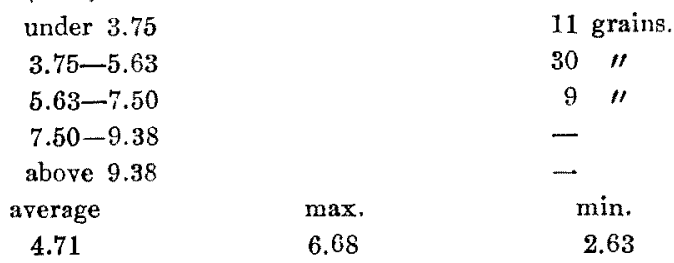

i) Volume of $100 \mathrm{~g} . \longrightarrow 116.0 \mathrm{c} . \mathrm{c}$.

j) Weight of 1,000 grains. $\quad 24.24 \mathrm{~g}$.

k) Volume of 1,000 grains.-16.8c.c.

n) Absorption of water. time steeped.

$$
2 \mathrm{~h} .
$$

$\%$ absorbed.

22.16

p) Chemical composition :-

$\begin{array}{lr}\text { Water } & 12.89 \\ \text { D. R. sugar } & 0.44 \\ \text { Starch } & 78.72 \\ \text { Crude fat } & 0.38 \\ \quad \text { " protein } & 7.20 \\ \text { Ash } & 0.33\end{array}$

$$
\text { unhydrous. }
$$$$
0.50
$$$$
89.51
$$$$
0.43
$$$$
8.27
$$

0.38

E) Korean rice.

a) Percentage of full grown grains.- 86.5

b) External appearance and luster.- Nearly same as C), but grains

\begin{tabular}{|c|c|c|c|c|c|}
\hline \multicolumn{2}{|c|}{ thickness abore } & 2.2 & $8.60 \mathrm{~g}$. & & rains. \\
\hline$\prime \prime$ & $\prime \prime$ & 2.0 & $27.38 \prime \prime$ & 14 & $\prime \prime$ \\
\hline$" \prime$ & $\prime \prime$ & 1.8 & $60.28, \prime$ & 30 & $\prime \prime$ \\
\hline$n$ & $\prime \prime$ & 1.6 & $3.16 \prime \prime$ & 2 & $\prime \prime$ \\
\hline "I & under & 1.6 & $0.63 / \prime$ & - & \\
\hline
\end{tabular}
were of middle size and not fat.

c) Size of grains. (m.m.) 


$\begin{array}{lccc} & \text { average. } & \text { max. } & \min \\ \text { length } & 4.90 & 5.60 & 4.45 \\ \text { width } & 2.72 & 3.10 & 2.35\end{array}$

d) Percentage of Shinziromai.- 50.7

e) Depth of longitudinal furrow. ( $/$ )

$\begin{array}{ccc}\text { average. } & \max , & \min . \\ 30.92 & 51.90 & 17.30\end{array}$

f) Specific gravity. -1.429

17.30

h) Hardness. kilo)

$$
\begin{aligned}
& \text { under } 3.75 \\
& 3.75-5.63 \\
& 5.63-7.50 \\
& 7.50-9.38 \\
& \text { above } 9.38 \\
& \text { average. }
\end{aligned}
$$$$
3.75
$$

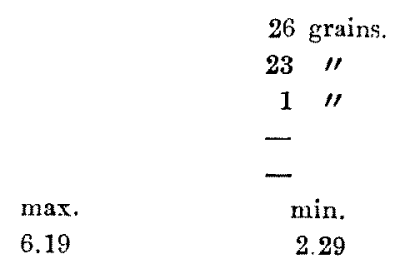

i) Volume of $100 \mathrm{~g} \cdot-116.5 \mathrm{c} . \mathrm{c}$.

j) Weight of 1,000 grains.-19.50c.c.

k) Volume of 1,000 grains. - 13.6c.c.

n) Absorption of water. time steeped.

$2 \mathrm{~h}$.

$\%$ absorbed. 19.9

p) Chemical composition :-

$\begin{array}{lrr}\text { Water } & 13.32 & - \\ \text { D. R. sugar } & 0.95 & 1.10 \\ \text { Starch } & 77.63 & 90.05 \\ \text { Crude fat } & 0.50 & 0.58 \\ \quad \text { " protein } & 7.30 & 8.47 \\ \text { Ash } & 0.31 & 0.36\end{array}$

1) Korean rice (Kokuryomiyako variety).

a) Percentage of full grown grains.- -86.6

b) External appearance and luster-_ Nearly same as A).

c) Size of grains. (m.m.)

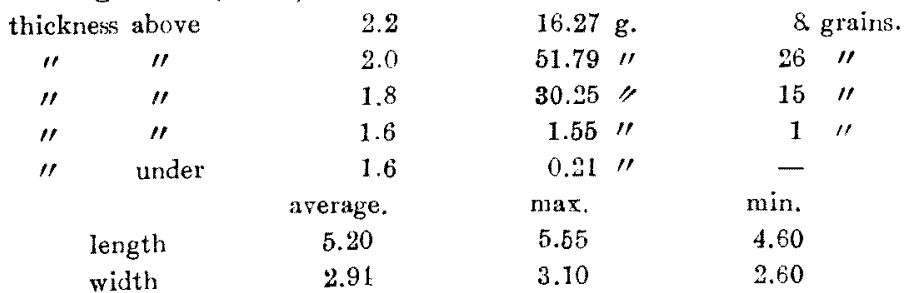

d) Percentage of Shinziromai.-- -87.0

e) Depth of longitudinal furrow. $(\mu)$ 


$\begin{array}{rrr}\text { average. } & \max . & \operatorname{min.} \\ 41.09 & 69.20 & 17.30\end{array}$

f) Specific gravity.- 1.492

h) Hardness. (kilo)

\begin{tabular}{|c|c|c|}
\hline under 3.75 & & 23 grairs. \\
\hline $3.75-5.63$ & & $18 \prime \prime$ \\
\hline $5.63-7.50$ & & $\prime \prime$ \\
\hline $7.50-9.38$ & & $\prime \prime$ \\
\hline above 9.38 & & - \\
\hline average. & $\max$. & min. \\
\hline 4.21 & 8.65 & 2.25 \\
\hline
\end{tabular}

i) Volume of $100 \mathrm{~g}$. - $114.0 \mathrm{c} . \mathrm{c}$.

j) Weight of 1,000 grains.-22.72g.

k) Volume of 1,000 grains. 15.70 c.c.

n) Absorption of water.

time steeped.

$2 \mathrm{~h}$.

$\%$ absorbed.

22.7

p) Chemical composition :-

$\begin{array}{lrc} & & \text { unhydrou } \\ \text { Water } & 11.85 & - \\ \text { D. R. sugar } & 0.65 & 0.73 \\ \text { Starch } & 79.90 & 90.28 \\ \text { Crude fat } & 0.29 & 0.33 \\ \quad \text { " protein } & 6.99 & 7.90 \\ \text { Ash } & 0.34 & 0.39\end{array}$

$(\mathrm{G}-\mathrm{U})$ omitter.

Polished rice unsuitable $(I-X)$ omitted.

$\left.\beta^{\prime}\right)$ Polished rice unfit ${ }_{f}$ for the Sake brewing.

I') Langoon rice.

a) Percentage of full grown grains.-51.1

b) External appearance and luster.—Greyish white colour, destitute of luster, grains were of middle size, abundant impurities were perceived.

c) Size of grains. (m.m.)

thickness above

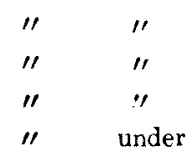

length

width

$7.68 \mathrm{~g}$.
$17.66 \prime \prime$
$46.64 \prime$
$17.60 "$
$10.28 \prime$
$\max$.
6.00
3.10

d) Percentage of Shinziromai. $-\quad 36.20$

f) Specific gravity. $\mathbf{1 . 4 2 9}$ 
h) Hardness. (kilo)

$$
\begin{aligned}
& \text { under } 3.75 \\
& 3.75-5.63 \\
& 5.63-7.50 \\
& 7.50-9.38 \\
& \text { above } 9.38 \\
& \text { average. } \\
& 3.65
\end{aligned}
$$

nax.

6.00

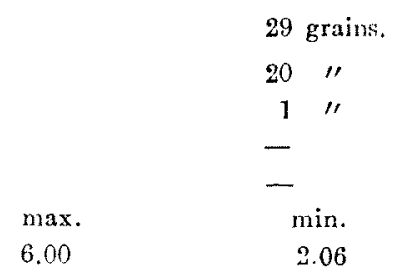

i) Volume of $100 \mathrm{~g} .-122.5 \mathrm{c} . \mathrm{c}$.

j) Weight of 1,000 grains. $-20.5 \mathrm{~g}$.

k) Volume of 1,000 grains.--13.65c.c.

n) Absorption of water. time steeped.

$2 \mathrm{~h}$. \% absorbed.

17.94

p) Chemical composition :-

\begin{tabular}{|c|c|c|c|c|c|c|}
\hline thickI & above & 2.2 & 3.40 & & 2 & grains. \\
\hline "I & $" \prime$ & 2.0 & 10.86 & $" \prime$ & 5 & $\prime \prime$ \\
\hline "I & " & 1.8 & 45.41 & "I & 23 & "I \\
\hline$" 1$ & "I & 1.6 & 23.73 & $\prime \prime$ & 12 & $" \prime$ \\
\hline$" \prime$ & under & 1.6 & 16.38 & $" \prime$ & 8 & $" \prime$ \\
\hline & & average. & $\max$. & & $\min$. & \\
\hline & gth & 5.52 & 5.80 & & 4.90 & \\
\hline & the & 2.42 & 2.80 & & 1.90 & \\
\hline
\end{tabular}

\begin{tabular}{lrc} 
Water & 12.96 & \multicolumn{1}{c}{-} \\
D. R. sugar & 0.44 & 0.50 \\
Starch & 79.73 & 90.89 \\
Crude fat & 0.48 & 0.55 \\
$\quad \prime$ protein & 6.01 & 6.85 \\
Ash & 0.43 & 0.49
\end{tabular}

II') Saigon rice.

a) Percentage of full grown grains. $-\mathbf{4 0 . 7 5}$

b) External appearance and luster. Nearly same as before, but grains

\begin{tabular}{|c|c|c|}
\hline under 3.75 & & It grains. \\
\hline $3.75-5.63$ & & $30 \prime \prime$ \\
\hline $5.63-7.50$ & & $\prime \prime$ \\
\hline $7.50-9.38$ & & - \\
\hline above 9.38 & & - \\
\hline average. & $\max$ & $\min$ \\
\hline
\end{tabular}
were more slender than the former and the impurities were abundant.

c) Size of grains. (m.m.)

d) Percentage of Shinziromai.- $\quad 29.86$

f) Specific gravity. $\quad 1.429$.

h) Hardness. (kilo) 
[Vol. 3,

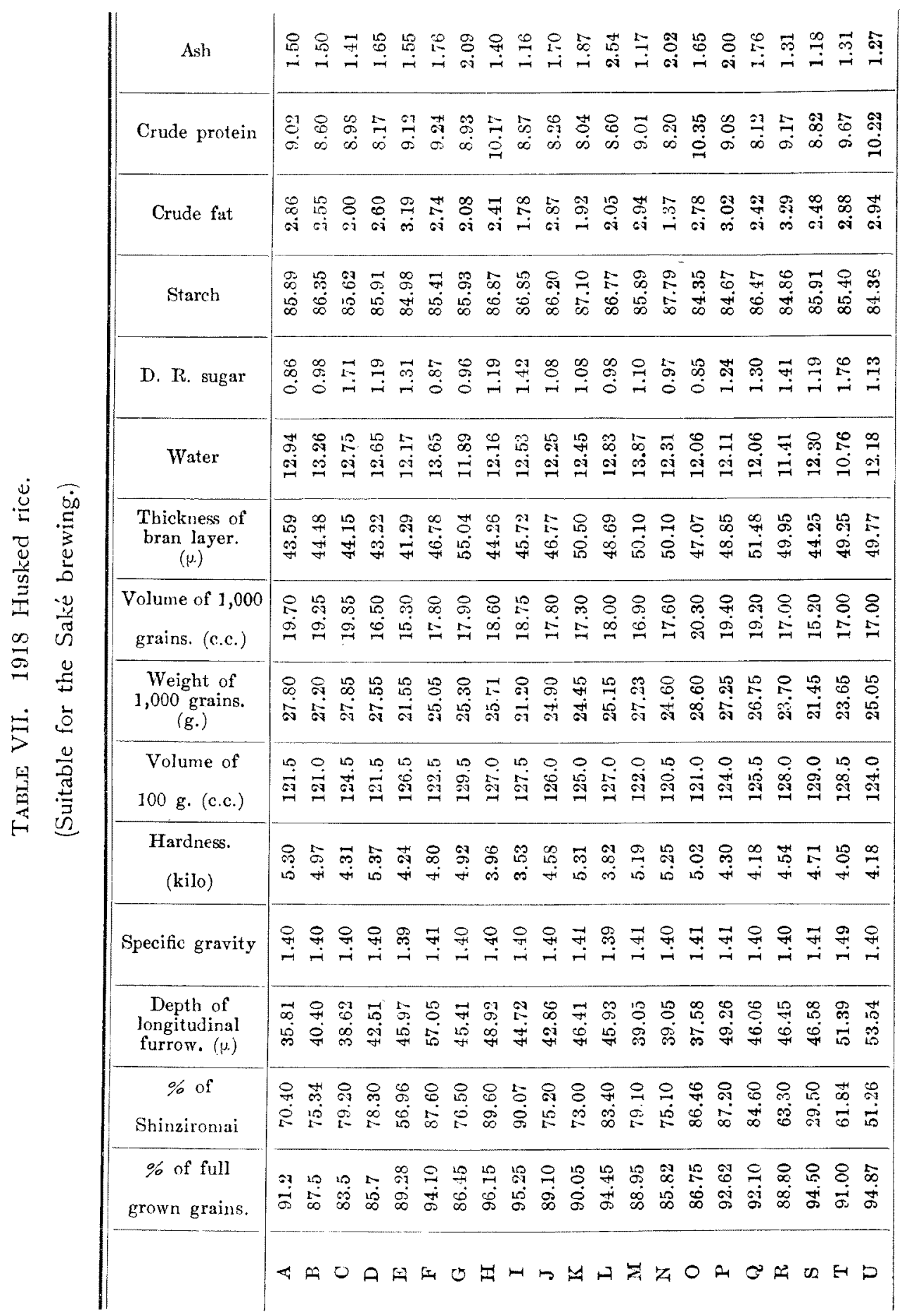




\begin{tabular}{|c|c|c|}
\hline & $\begin{array}{l}\text { Absorption } \\
\text { of water. (\%) }\end{array}$ & 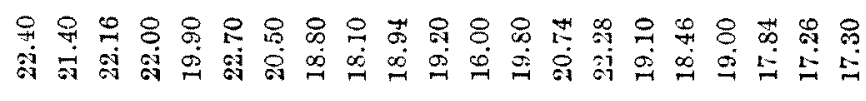 \\
\hline & Ash & 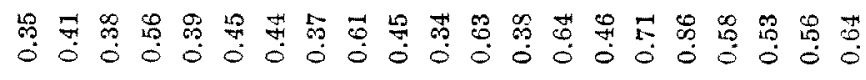 \\
\hline & Crude protein & 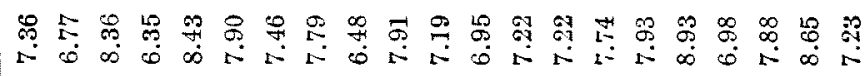 \\
\hline & Crude fat & 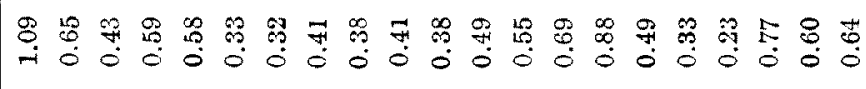 \\
\hline & Starch & 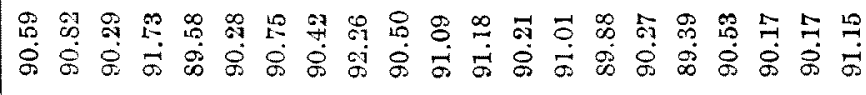 \\
\hline & D. R. Sugar & 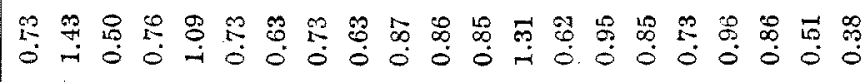 \\
\hline 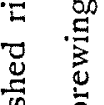 & Water & 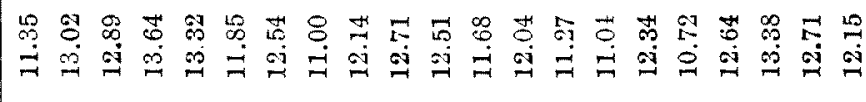 \\
\hline$\sum_{-1}^{\infty} \frac{-4}{\tilde{N}}$ & $\begin{array}{l}\text { Volume of } 1,000 \\
\text { grains. (c c.) }\end{array}$ & 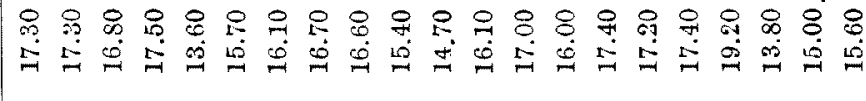 \\
\hline$\frac{g}{2}$ & $\begin{array}{l}\text { Weight of } \\
1,000 \text { grains. } \\
(\mathrm{g} .)\end{array}$ & 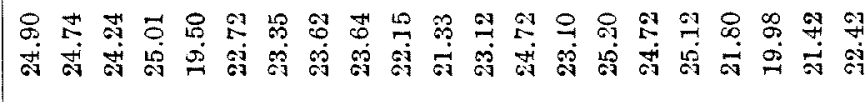 \\
\hline$\stackrel{\frac{\Delta}{0}}{\stackrel{\pi}{*}}$ & $\begin{array}{c}\text { Volume of } 100 \mathrm{~g} \\
\text { (c.c.) }\end{array}$ & 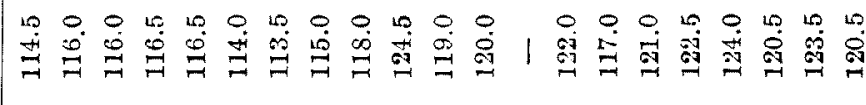 \\
\hline & $\begin{array}{l}\text { Mardness. } \\
\qquad \text { (kilo) }\end{array}$ & 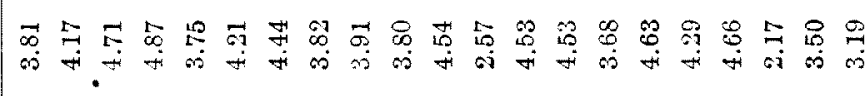 \\
\hline & Specific gravity & 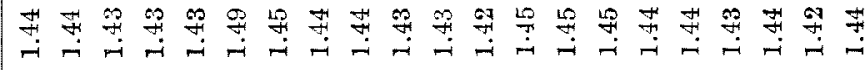 \\
\hline & $\begin{array}{l}\text { Depth of } \\
\text { longitudinal } \\
\text { furrow. }(\mu)\end{array}$ & 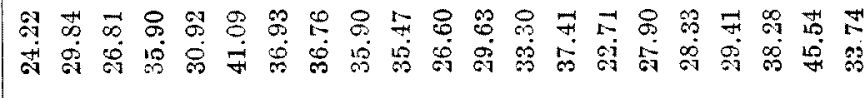 \\
\hline & $\begin{array}{c}\% \text { of } \\
\text { Shinziromai }\end{array}$ & 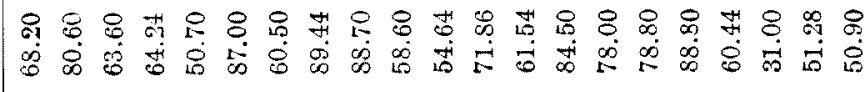 \\
\hline & $\begin{array}{l}\% \text { of full } \\
\text { grown grains. }\end{array}$ & 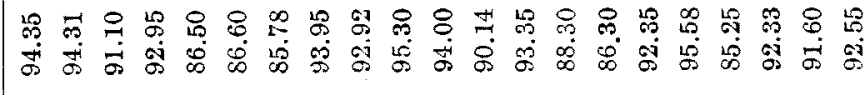 \\
\hline & & 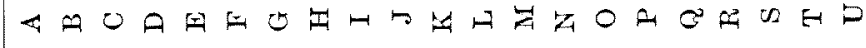 \\
\hline
\end{tabular}




\begin{tabular}{|c|c|c|c|c|c|c|c|c|c|c|c|}
\hline & Ash & 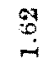 & 管 & $\stackrel{\mathscr{m}}{\rightarrow}$ & 菅 & $\stackrel{\infty}{\stackrel{-}{-}}$ & 嵌 & $\begin{array}{l}\infty \\
\stackrel{0}{0} \\
0\end{array}$ & గִ & ن & $\underset{\stackrel{\mathscr{g}}{\mathrm{I}}}{\stackrel{\mathscr{P}}{2}}$ \\
\hline & Crude protein & $\stackrel{\infty}{\Rightarrow}$ & $\stackrel{m}{0}$ & $\begin{array}{l}\text { 瓷 } \\
\text { con }\end{array}$ & $\begin{array}{l}\text { హ్ } \\
\text { क }\end{array}$ & $\begin{array}{l}: 0 \\
\infty \\
\infty \\
\infty\end{array}$ & $\begin{array}{l}\stackrel{8}{:} \\
\infty \\
\infty\end{array}$ & $\begin{array}{l}\text { హ్ } \\
\text { హ) }\end{array}$ & $\begin{array}{l}1 \\
\infty \\
\infty \\
\infty\end{array}$ & 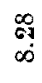 & $\begin{array}{l}\vec{d} \\
\dot{c}\end{array}$ \\
\hline & Crude fat & $\begin{array}{l}\stackrel{\infty}{\circ} \\
\text { a }\end{array}$ & 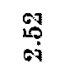 & $\begin{array}{l}\text { 㔛 } \\
\text { oi }\end{array}$ & $\stackrel{5}{\stackrel{5}{9}}$ & 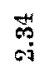 & $\stackrel{\infty}{\rightarrow}$ & $\hat{i}$ & 昂 & $\begin{array}{l}8 \\
0 . j\end{array}$ & $\begin{array}{l}0 \\
\text { : } \\
\text { ij }\end{array}$ \\
\hline & Starch & $\begin{array}{l}\infty \\
\infty \\
0 \\
0 \\
\infty\end{array}$ & $\overbrace{\infty}^{10}$ & $\begin{array}{l}m \\
0 \\
0 \\
\infty\end{array}$ & $\underset{\infty}{\stackrel{2}{\infty}}$ & $\begin{array}{l}\infty \\
\vdots \\
\infty \\
\infty\end{array}$ & $\begin{array}{l}5 \\
50 \\
10 \\
10\end{array}$ & 索 & $\begin{array}{l}\infty \\
\infty \\
\infty \\
\infty \\
\infty\end{array}$ & $\underset{\substack{: \\
\infty}}{\stackrel{0}{\infty}}$ & $\begin{array}{l}\overrightarrow{6} \\
1: 0 \\
\infty\end{array}$ \\
\hline & D. R. sugar & $\underset{-i}{\infty}$ & 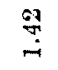 & $\stackrel{8}{\circ}$ & $\begin{array}{l}\mathscr{8} \\
0 \\
0\end{array}$ & $\stackrel{10}{2}$ & $\underset{\text { oi }}{\stackrel{0}{0}}$ & $\stackrel{5}{\circ}$ & 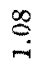 & $\begin{array}{l}\mathscr{0} \\
: \\
0\end{array}$ & م. \\
\hline & Water & $\underset{\sim}{\stackrel{n}{\sim}}$ & $\begin{array}{c}\text { in } \\
\text { gij } \\
\end{array}$ & 总 & $\stackrel{8}{\stackrel{8}{+}}$ & 查 & 率 & $\stackrel{0}{\stackrel{0}{二}}$ & 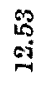 & 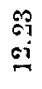 & 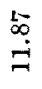 \\
\hline E & $\begin{array}{l}\text { Thickness of } \\
\text { bran layer. ( } \mu \text { ) }\end{array}$ & 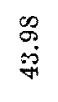 & 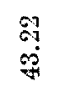 & 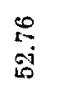 & $\begin{array}{l}\vec{\sigma} \\
\dot{5}\end{array}$ & 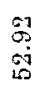 & 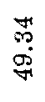 & $\frac{a}{c i}$ & $\stackrel{\circ}{\stackrel{9}{\circ}}$ & 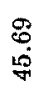 & 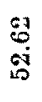 \\
\hline तू & $\begin{array}{c}\text { Volume of } \mathrm{J}, 000 \\
\text { grains. (c.c.) }\end{array}$ & $\begin{array}{l}0 \\
0 \\
0 \\
0\end{array}$ & $\stackrel{ }{0}$ & $\begin{array}{l}8 \\
\stackrel{9}{0}\end{array}$ & $\stackrel{0}{:}$ & \begin{tabular}{l}
0 \\
0 \\
\hdashline
\end{tabular} & $\begin{array}{l}8 \\
9 \\
0\end{array}$ & \begin{tabular}{l}
8 \\
$\stackrel{8}{4}$ \\
0 \\
\hdashline
\end{tabular} & 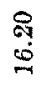 & 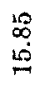 & 足 \\
\hline b & $\begin{array}{l}\text { Weight of } \\
1,000 \text { grains. } \\
\text { (g.) }\end{array}$ & $\stackrel{0}{\sim}$ & 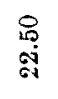 & $\underset{\substack{R \\
g i}}{c}$ & $\stackrel{12}{=}$ & $\begin{array}{l}8 \\
8 \\
81\end{array}$ & 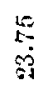 & $\stackrel{9}{\stackrel{9}{\leftrightarrow: ~}}$ & $\begin{array}{l}10 \\
2\end{array}$ & 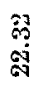 & $\frac{\text { 㔖 }}{\stackrel{4}{+}}$ \\
\hline 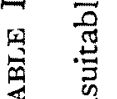 & $\begin{array}{l}\text { Volume of } 100 \mathrm{~g} \text {. } \\
\text { (c.c.) }\end{array}$ & 渵 & 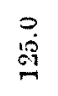 & 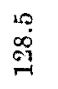 & $\stackrel{\circ}{\stackrel{9}{9}}$ & 兽 & 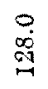 & $\begin{array}{c}\circ \\
\text { : } \\
\stackrel{\infty}{\infty}\end{array}$ & 崖 & 战 & 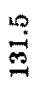 \\
\hline & Hardness. (kilo) & $\underset{10}{\circ}$ & 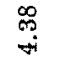 & $\stackrel{\vec{\sigma}}{\vec{d}}$ & ד. & : & $\begin{array}{l}\text { के } \\
\text { is }\end{array}$ & $\overrightarrow{~ क ् ष े ~}$ & f & $\stackrel{9}{10}$ & $\underset{10}{\stackrel{9}{0}}$ \\
\hline & Specific gravity & $\stackrel{?}{\rightarrow}$ & $\underset{\text { 암 }}{\text {. }}$ & $\underset{+}{\vec{H}}$ & 勌 & 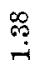 & & 象 & 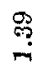 & 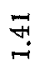 & $\underset{-}{-}$ \\
\hline & $\begin{array}{l}\text { Depth of } \\
\text { longitudinal } \\
\text { furrow. }(\mu)\end{array}$ & $\begin{array}{l}\overrightarrow{0} \\
10 \\
100\end{array}$ & $\begin{array}{l}9 \\
0 \\
5 \\
5\end{array}$ & $\frac{g}{7}$ & 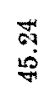 & 孚 & $\begin{array}{l}50 \\
50 \\
100\end{array}$ & $\underset{\substack{* \\
+}}{\sharp}$ & 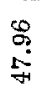 & $\stackrel{?}{*}$ & 总 \\
\hline & $\begin{array}{c}\% \text { of } \\
\text { Shinziromai. }\end{array}$ & 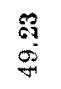 & $\stackrel{8}{\oplus}$ & $\begin{array}{l}8 \\
\infty \\
\infty \\
1 \\
0\end{array}$ & $\begin{array}{l}80 \\
\text { in }\end{array}$ & $\stackrel{\circ}{i-1}$ & $\begin{array}{l}8 \\
8 \\
8\end{array}$ & 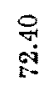 & $\begin{array}{l}8 \\
\text { gi } \\
\text { gi }\end{array}$ & 总 & 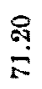 \\
\hline & $\begin{array}{l}\% \text { of full } \\
\text { grown grains. }\end{array}$ & $\begin{array}{l}\qquad \\
\stackrel{8}{0} \\
\infty\end{array}$ & $\stackrel{2}{\rightleftarrows}$ & $\begin{array}{l}\text { 옹 } \\
\text { सं }\end{array}$ & $\begin{array}{l}R \\
8 \\
8\end{array}$ & 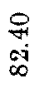 & $\underset{2}{\stackrel{0}{2}}$ & $\stackrel{5}{10}$ & $\begin{array}{l}20 . \\
\rightarrow 1 \\
\infty \\
\infty\end{array}$ & $\begin{array}{l}10 \\
10 \\
0 \\
0\end{array}$ & $\begin{array}{l}\frac{1}{\infty} \\
\infty \\
8\end{array}$ \\
\hline & & - & $\Xi$ & $E$ & $Z$ & $>$ & 5 & $\stackrel{5}{S}$ & & 省 & $M$ \\
\hline
\end{tabular}




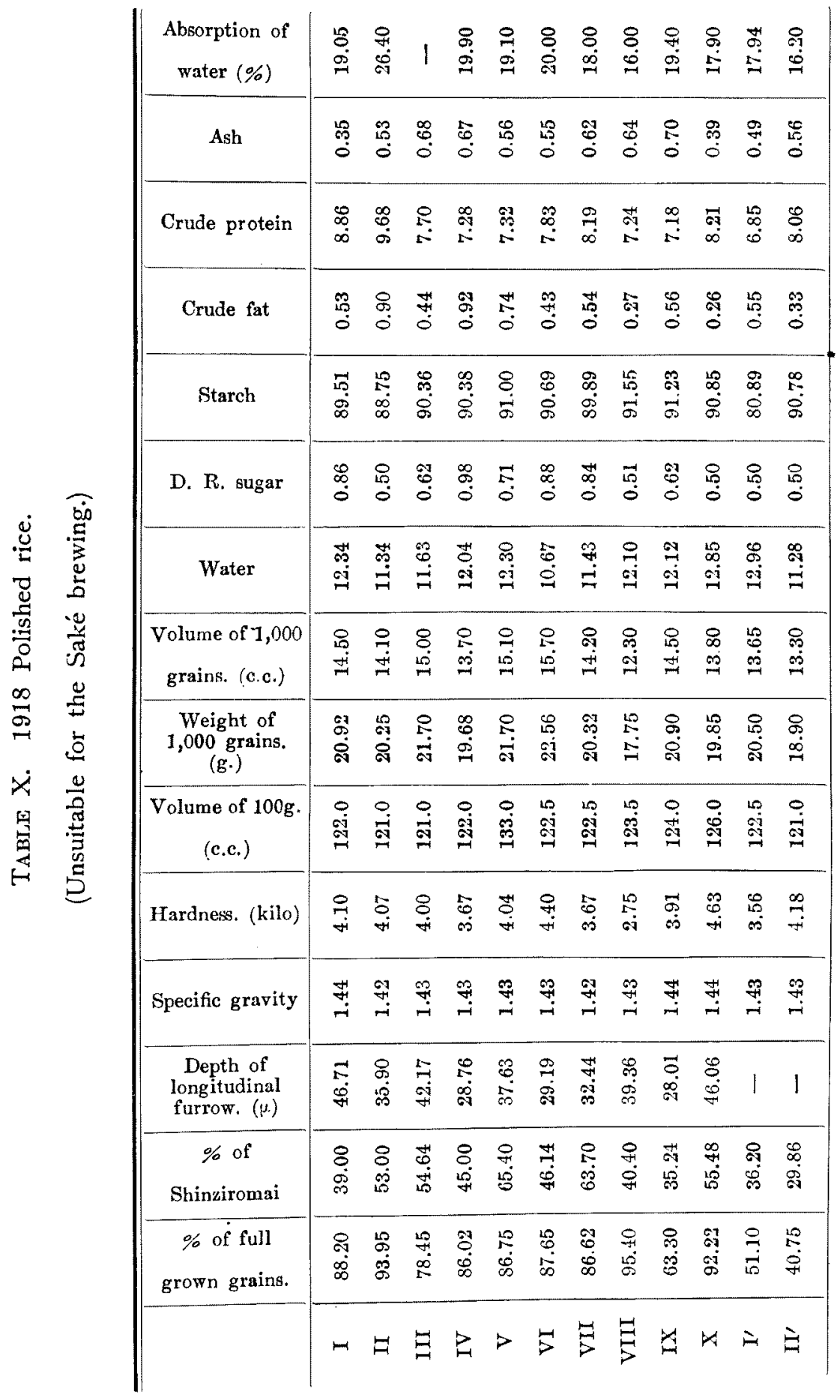


i) Volume of $100 \mathrm{~g}$. - 121.0c.c.

j) Weight of 1,000 grains. $-18.90 \mathrm{~g}$.

k) Volume of 1,000 grains.-13.3c.c.

n) Absorption of water.

time steeped.

$2 \mathrm{~h}$.

$\%$ absorbed.

16.2

p) Chemical composition.-

$\begin{array}{lrr}\text { Water } & 11.28 & - \\ \text { D. R. sugar } & 0.45 & 0.50 \\ \text { Starch } & 80.34 & 90.78 \\ \text { Crude fat } & 0.29 & 0.33 \\ \quad \text { " protein } & 7.14 & 8.06 \\ \text { Ash } & 0.50 & 0.56\end{array}$

\section{Summary of the 1918 production.}

In the suitable materials for the Sake brewing, percentages of full grown grains, and shinziromai, size of grains, specific gravity, both volume and weight of 1,000 grains and starch showed a higher value than the unsuitable, while depth of longitudinal furrow, volume of $100 \mathrm{~g}$. thickness of bran layer, water, sugar, crude fat, crude protein and ash showed a smaller value. External appearance and luster should be fine.

\section{Production.}

a) Husked rice, suitable for the Sake brewing. 19 (A-S) varieties.

A) Production of Okayama prefecture.

a) Percentage of full grown grains. -90.0

b) External appearance and luster.- - Light straw yellow waxy luster, grains were fat and large. (Ist class).

c) Size of grains. (m.m.)

$\begin{array}{ccr}\text { thickness above } & 2.2 \\ \prime \prime & \text { " } & 2.0 \\ \text { " } & \text { " } & 1.8 \\ " \text { under } & 1.8 \\ & & \text { average. } \\ & \text { length } & 5.04 \\ & \text { width } & 3.02\end{array}$

$\begin{array}{cc}77.60 \mathrm{~g} . & 39 \text { grains. } \\ 17.25 \prime \prime & 9 \prime \prime \\ 4.80 " \prime & 2 \prime \prime \\ 0.30 " \prime & - \\ \max . & \text { min. } \\ 5.80 & 5.00 \\ 3.30 & 2.65\end{array}$

d) Percentage of Shinziromai._-13.3

e) Depth of longitudinal furrow. $(\mu)$

$\begin{array}{ccc}\text { average. } & \max . & \min . \\ 57.52 & 95.15 & 25.95\end{array}$

f) Specific gravity.-1.418 
h) Hardness. (kilo)

$$
\begin{array}{ccc}
\text { average. } & \max . & \min . \\
607 & 9.38 & 3.90
\end{array}
$$

i) Volume of $100 \mathrm{~g}$.- $121.0 \mathrm{c} . \mathrm{c}$.

j) Weight of 1,000 grains. $26.9 \mathrm{~g}$.

k) Volume of 1,000 grains.-19.0c.c.

m) Thickness of bran layer. $(\mu)$

$\begin{array}{rrr}\text { average. } & \max . & \min . \\ 33.30 & 60.55 & 17.30\end{array}$

p) Chemical composition.--

$\begin{array}{lr}\text { Water } & 1246 \\ \text { Starch } & 72.03 \\ \text { Sugar } & 0.71 \\ \text { Crude protein } & 8.52 \\ \quad " \text { fat } & 2.24 \\ \text { Ash } & 1.28\end{array}$

B) Production of Hyogo prefecture.

a) Percentage of full grown grains.- -98.6 class).

b) External appearance and luster.—Nearly same as the former (lst

\begin{tabular}{|c|c|c|c|c|c|}
\hline thickness & above & 2.2 & $77.70 \mathrm{~g}$ & 39 & grains \\
\hline$\prime \prime$ & $\prime \prime$ & 2.0 & $19.90 "$ & 10 & $\prime \prime$ \\
\hline$" \prime$ & $" 1$ & 1.8 & $2.40 \prime \prime$ & 1 & " \\
\hline$" \prime$ & under & $\begin{array}{c}1.8 \\
\text { average. }\end{array}$ & $\begin{array}{l}0.10 " \\
\text { ruax. }\end{array}$ & $\overline{\min }$ & \\
\hline & ggth & 5.38 & 5.70 & 4.90 & \\
\hline & dth & 3.12 & 3.55 & 2.90 & \\
\hline
\end{tabular}

c) Size of grains. (m.m.)

d) Percentage of Shinziromai._- $91.6 \%$

e) Depth of longitudinal furrow. $(\mu)$

average. $\quad$ max. $\min$.

$\begin{array}{lll}57.31 & 86.50 & 34.60\end{array}$

f) Specific gravity.- 1.399

h) Hardness. (kilo)

$\begin{array}{ccc}\text { average. } & \max . & \min . \\ 4.80 & 7.69 & 3.43\end{array}$

i) Volume of $100 \mathrm{~g}-125.0$ c.c.

j) Weight of 1,000 grains.-27.1g.

k) Volume of 1,000 grains.-19.4c.c.

$\mathrm{m})$ Thickness of bran layer. $(\mu)$

average.

42.22 max.

47.93 $\min$.

37.80 
p) Chemical composition :-

$\begin{array}{lr}\text { Water } & 14.23 \\ \text { Starch } & 74.12 \\ \text { Sugar } & 0.90 \\ \text { Crude protein } & 10.13 \\ \text { " fat } & 1.72 \\ \text { Ash } & 1.03\end{array}$

C) Production of Hyogo prefecture.

a) Percentage of full grown grains.- 98.3

b) External appearance and luster.-Same as B).

c) Size of grains. (m.m.)

\begin{tabular}{|c|c|c|c|c|}
\hline thickness & above. & 2.2 & $55.5 \mathrm{~g}$ & 28 \\
\hline$" \prime$ & $" \prime$ & 2.0 & 39.2, & 20 \\
\hline " & " & 1.8 & 5.3 & 2 \\
\hline$"$ & under & $\begin{array}{r}1.8 \\
\text { average. }\end{array}$ & $\begin{array}{c}0 \\
\max .\end{array}$ & $\overline{\min }$ \\
\hline & ggth & 5.29 & 5.70 & 4.80 \\
\hline wid & dth & 3.17 & 3.40 & 3.00 \\
\hline
\end{tabular}

d) Percentage of Shinziromai._-92.6

e) Depth of longitudinal furrow. $(\mu)$

$\begin{array}{rrr}\text { average. } & \max . & \min . \\ 59.25 & 86.50 & 34.60\end{array}$

f) Specific gravity. -1.399

h) Hardness. (kilo)

$\begin{array}{ccc}\text { average. } & \max . & \min . \\ 5.63 & 10.80 & 4.09\end{array}$

i) Volume of $100 \mathrm{~g} .-121.0 \mathrm{c} . \mathrm{c}$.

j) Weight of 1,000 grains. $-26.4 \mathrm{~g}$.

k) Volume of 1,000 grains.-18.7c.c.

m) Thickness of bran layer. $(\mu)$

$\begin{array}{rrr}\text { average. } & \max . & \text { nin. } \\ 44.35 & 51.08 & 40.95\end{array}$

p) Chemical composition :-

$\begin{array}{lr}\text { Water } & 11.95 \\ \text { Starch } & 70.08 \\ \text { Sugar } & 1.53 \\ \text { Crude protein } & 9.69 \\ \text { " fat } & 1.91 \\ \text { Ash } & 1.19\end{array}$

D) Production of Osaka prefecture.

a) Percentage of full grown grains, 97.0

b) External appearance and luster.-Same as B) 
c) Size of grains. (m.m.)

\begin{tabular}{|c|c|c|c|c|c|}
\hline thickness & above & 2.2 & $83.15 \mathrm{~g}$. & $42 \mathrm{~g}$ & rains \\
\hline$"$ & " & 2.0 & $14.55 "$ & 7 & " \\
\hline " & " & 1.8 & $2.25 \mathrm{\prime \prime}$ & 1 & " \\
\hline " & under & 1.8 & $0.05 \prime \prime$ & - & \\
\hline & & average. & max. & $\min$. & \\
\hline & ngth & 5.34 & 5.65 & 5.10 & \\
\hline & idth & 3.15 & 3.35 & 2.90 & \\
\hline
\end{tabular}

d) Percentage of Shinziromai. -80.7

e) Depth of longitudinal furrow. $(\mu)$

$\begin{array}{ccc}\text { average. } & \max . & \min . \\ 53.85 & 86.50 & \mathbf{3 4 . 6 0}\end{array}$

f) Specific gravity. ' 1.409

h) Hardness. (kilo)

$\begin{array}{ccc}\text { average. } & \max . & \min . \\ 4.64 & 7.88 & 3.26\end{array}$

i) Volume of 100g. - 124.0c.c.

j) Weight of 1,000 grains. $-27.3 \mathrm{~g}$.

k) Volume of 1,000 grains.-19.3c.c.

$\mathrm{m})$ Thickness of bran layer. $(\mu)$

average. $\max$ min.

$\begin{array}{lll}53.85 & 86.50 & 34.60\end{array}$

p) Chemical composition.-

$\begin{array}{lr}\text { Water } & 12.82 \\ \text { Starch } & 73.05 \\ \text { Sugar } & 0.83 \\ \text { Crude protein } & 9.25 \\ \quad \text { " fat } & 1.51 \\ \text { Ash } & 0.95\end{array}$

E) Production of Saga prefecture.

a) Percentage of full grown grains.- 93.4

b) External appearance and luster._- Same as A)

c) Size of grains. (m.m.)

$\begin{array}{cccc}\text { thickness above } & 2.2 & 56.55 \mathrm{~g} . & 28 \text { grains. } \\ \prime \prime \prime \prime \prime & 2.0 & 35.50 " & 18 " \text { " }\end{array}$




\begin{tabular}{|c|c|c|c|}
\hline$\prime \prime$ & 1.8 & $7.15 \prime \prime$ & 4 \\
\hline under & 1.8 & $0.60 \prime \prime$ & - \\
\hline & average. & $\max$. & min. \\
\hline length & 5.15 & 5.50 & 4.90 \\
\hline width & 3.09 & 3.25 & 2.90 \\
\hline
\end{tabular}

d) Percentage of Shinziromai.- 5.8

e) Depth of longitudinal furrow. $(\mu)$

$\begin{array}{rrr}\text { average. } & \max . & \min . \\ 59.25 & 86.50 & 34.60\end{array}$

f) Specific gravity. $\mathbf{1 . 4 0 9}$

h) Hardness. (kilo)

$\begin{array}{ccc}\text { average. } & \max . & \min . \\ 6.12 & 9.28 & 3.45\end{array}$

i) Volume of $100 \mathrm{~g} . \longrightarrow 125.0 \mathrm{c} . \mathrm{c}$.

j) Weight of 1,000 grains.—24.45g.

k) Volume of 1,000 grains.-17.15c.c.

$\mathrm{m})$ Thickness of bran layer. $(\mu)$

$\begin{array}{ccc}\text { average. } & \max . & \mathrm{min} . \\ 41.34 & 45.45 & 31.73\end{array}$

p) Chemical composition :-

$\begin{array}{lr}\text { Water } & 12.94 \\ \text { Starch } & 72.09 \\ \text { Sugar } & 0.65 \\ \text { Crude protein } & 9.11 \\ \quad \text { "fat } & 2.40 \\ \text { Ash } & 1.29\end{array}$

F) Production of Kumamoto prefecture.

a) Percentage of full grown grains.-96.5

b) External appearance and luster.-Same as before.

c) Size of grains. (m.m.)

$\begin{array}{cccccc}\text { thickness above } & 2.2 & 65.50 \mathrm{~g} . & 33 \text { grains. } \\ \prime \prime & \prime \prime & 2.0 & 26.60 " & 13 " \\ \prime \prime & \prime \prime & 1.8 & 7.20 " & 4 & \prime \prime \\ \prime \prime & \text { under } & 1.8 & 0.60 " & - & \end{array}$




$\begin{array}{lccc} & \text { average. } & \text { max. } & \text { min. } \\ \text { length } & 5.12 & 5.45 & 4.85 \\ \text { width } & 3.09 & 3.35 & 2.75\end{array}$

d) Percentage of Shinziromai.-4.6

e) Depth of longitudinal furrow. $(\mu)$

$\begin{array}{ccc}\text { average. } & \max . & \min . \\ 54.71 & 86.50 & 17.30\end{array}$

f) Specific gravity. -1.399

h) Hardness. (kilo)

$\begin{array}{ccc}\text { average. } & \max . & \min . \\ 6.17 & 9.04 & 3.75\end{array}$

i) Volume of $100 \mathrm{~g}$. 122.0c.c.

j) Weight of 1,000 grains. $-24.8 \mathrm{~g}$.

k) Volume of 1,000 grains.-17.7c.c.

m) Thickness of bran layer. $(\mu)$

$\begin{array}{ccc}\text { average. } & \max . & \min . \\ 39.88 & 45.23 & \mathbf{3 3 . 0 8}\end{array}$

p) Chemical composition :-

$\begin{array}{lr}\text { Water } & 13.87 \\ \text { Starch } & 74.28 \\ \text { Sugar } & 0.71 \\ \text { Crude protein } & 8.82 \\ \text { " fat } & 1.96 \\ \text { Ash } & 1.23\end{array}$

G) Production of Hiroslima prefecture.

a) Percentage of full grown grains._-93.3

b) External appearance and luster.-Same as B).

c) Size of grains. (m.m.)

\begin{tabular}{|c|c|c|c|c|c|}
\hline thickness & above & 2.2 & $75.70 \mathrm{~g}$. & \multicolumn{2}{|c|}{38 grains } \\
\hline " & $" \prime$ & 2.0 & $18.90 \prime \prime$ & 10 & $\prime \prime$ \\
\hline ' & $\prime \prime$ & 1.8 & $4.75 \|$ & 2 & $" \prime$ \\
\hline " & under & 1.8 & $0.65 \prime \prime$ & - & $\prime \prime$ \\
\hline & & average. & $\max$ & $\min$. & \\
\hline & agth & 5.21 & 5.60 & 4.90 & \\
\hline & dth & 3.09 & 3.35 & 2.90 & \\
\hline
\end{tabular}


d) Percentage of Shinziromai- $47.10 \%$

e) Depth of longitudinal furrow. $(\mu)$

$\begin{array}{ccc}\text { average. } & \max . & \min . \\ 49.31 & 69.20 & 25.95\end{array}$

f) Specific gravity.- 1.399

h) Hardness. (kilo)

$\begin{array}{ccc}\text { average. } & \max . & \min . \\ 5.22 & 7.69 & 3.56\end{array}$

i) Volume of 100g._-124.0c.c.

j) Weight of 1,000 grains. $-25.6 \mathrm{~g}$.

k) Volume of 1,000 grains.-18.1c.c.

m) Thickness of bran layer. $(\mu)$

$\begin{array}{ccc}\text { average. } & \max . & \min . \\ 42.33 & 65.13 & 36.23\end{array}$

p) Chemical composition :-

$\begin{array}{lr}\text { Water } & 12.80 \\ \text { Starch } & 71.98 \\ \text { Sugar } & 0.77 \\ \text { Crude protein } & 9.69 \\ \quad \text { 'fat } & \mathbf{2 . 1 8} \\ \text { Ash } & 1.21\end{array}$

H) Production of Olayama prefecture.

a). Percentage of full grown grains. -83.7

b) External appearance and iuster.- Nearly same as G).

c) Size of grains. (m.m.)

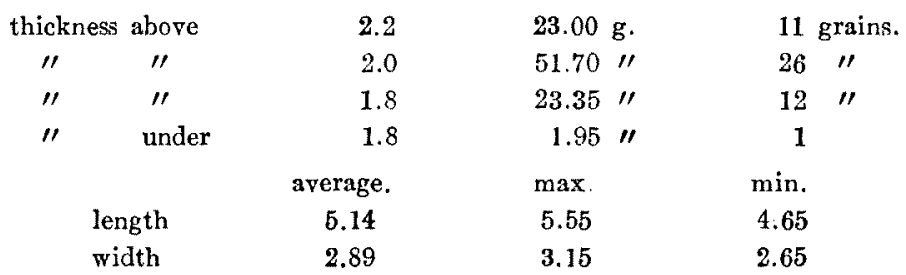

d) Percentage of Shinziromai. -1.5

e) Depth of longitudinal furrow. $(\mu)$

$\begin{array}{ccc}\text { average. } & \max . & \min . \\ 53.20 & 86.50 & 17.30\end{array}$


f) Specific gravity.-1.385

h) Hardness. (kilo)

$\begin{array}{ccc}\text { average. } & \max . & \min . \\ 5.83 & 7.61 & \mathbf{3 . 2 6}\end{array}$

i) Volume of $100 \mathrm{~g} . \longrightarrow 124.0 \mathrm{c} . \mathrm{c}$.

j) Weight of 1,000 grains.-24.2g.

k) Volume of 1,000 grains. - 17.1c.c.

m) Thickness of bran layer. $(k)$

$\begin{array}{ccc}\text { average. } & \max . & \min . \\ 43.29 & 49.28 & \mathbf{3 6 . 0 0}\end{array}$

p) Chemical composition :-

$\begin{array}{lr}\text { Water } & 12.86 \\ \text { Starch } & 70.74 \\ \text { Sugar } & 0.90 \\ \text { Crude protein } & 7.34 \\ \quad \text { fat } & 2.10 \\ \text { Ash } & 1.21\end{array}$

I) Production of Yamaguchi prefecture.

a) Percentage of full grown grains. -92.8

b) External appearance and luster.- Same as $\mathrm{H}$ ).

c) Size of grains. (m.m.)

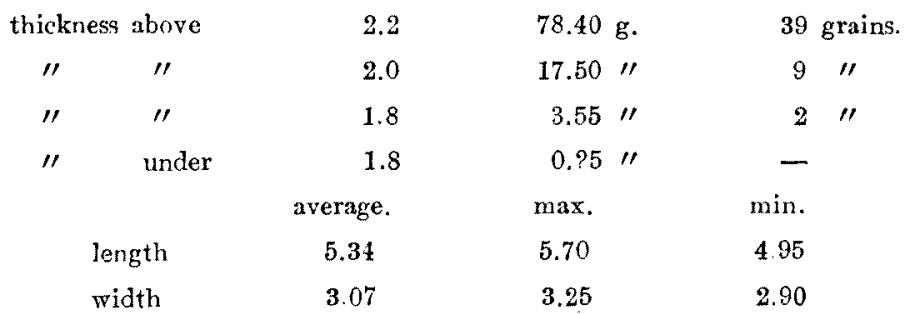

d) Percentage of Shinziromai. $-9.5 \%$

e) Depth of longitudinal furrow. $(\mu)$

$\begin{array}{ccc}\text { average. } & \max . & \min . \\ 47.79 & 86.50 & 17.30\end{array}$

f) Specific gravity.- -1.404 
h) Hardness. (kilo)

$\begin{array}{ccc}\text { average. } & \text { max. } & \text { min. } \\ 6.50 & 10.14 & 3.38\end{array}$

i) Volume of $100 \mathrm{~g}$. - $120.5 \mathrm{c} . \mathrm{c}$.

j) Weight of 1,000 grains. $-26.6 \mathrm{~g}$.

k) Volume of 1,000 grains.—-18.75c.c.

m) Thickness of bran layer. $(\mu)$

$\begin{array}{ccc}\text { average. } & \text { max. } & \text { min. } \\ \mathbf{4 2 . 5 5} & 47.25 & 36.68\end{array}$

p) Chemical composition :-

$\begin{array}{lr}\text { Water } & 12.94 \\ \text { Stareh } & 72.99 \\ \text { Sugar } & 0.90 \\ \text { Crude protein } & 9.10 \\ \quad \text { "fat } & 2.06 \\ \text { Ash } & 1.04\end{array}$

J) Production of Niigata prefecture.

a) Percentage of full grown grains._-95.3

b) External appearance and luster. - Straw yellow colour, grains were fat and of middle size, but somewhat poor of luster (2nd class).

c) Size of grains. (m.m.)

thickness above

" "

" $"$

"under

length
width

2.2

3.0

1.8

1.8

average.

5.14

2.89
$23.0 \mathrm{~g}$

$51.7 \prime \prime$

23.35 "

$1.95 \prime \prime$

$\max$.

5.55

3.15
11 grains.

26 "

12 "

1

min.

4.65

2.65

d) Percentage of Shinziromai. -0.6

e) Depth of longitudinal furrow. $(\mu)$

$\begin{array}{rrr}\text { average } & \max . & \min . \\ 44.33 & 95.15 & 17.30\end{array}$

f) Specific gravity.-1.389 
h) Hardness. (kilo)

$\begin{array}{ccc}\text { average. } & \text { max. } & \text { nin. } \\ 5.55 & 7.13 & 4.03\end{array}$

i) Volume of $100 \mathrm{~g} .-129.0 \mathrm{c} . \mathrm{c}$.

j) Weight of 1,000 grains. $--22.4 \mathrm{~g}$.

k) Volume of 1,000 grains. - 16.0c.c.

ii) Thickness of bran layer. $(\mu)$

$\begin{array}{ccc}\text { average. } & \max . & \min . \\ 44.33 & 95.15 & 17.30\end{array}$

p) Chemical composition :-

$\begin{array}{lr}\text { Water } & 13.89 \\ \text { Starch } & 70.43 \\ \text { Sugar } & 0.65 \\ \text { Crude protein } & 8.81 \\ \quad \text { "fat } & 2.00 \\ \text { Ash } & 1.08\end{array}$

K) Production of Aichi prefecture.

a) Percentage of full grown grains. 93.5

b) External appearance and luster.—Same as J).

c) Size of grains. (m.m.)

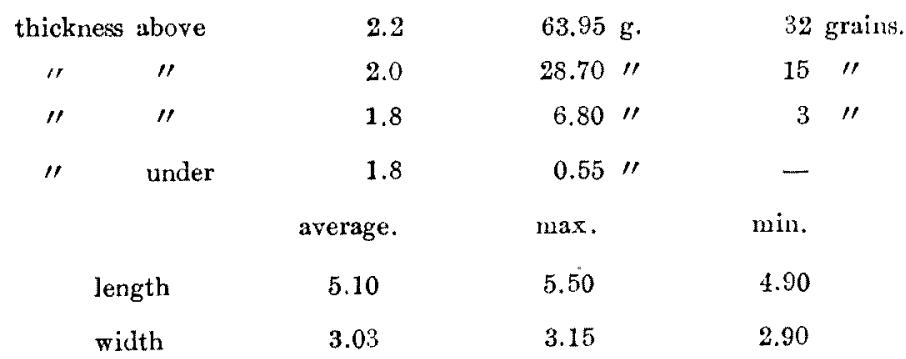

d) Percentage of Shinziromai.-3.2

e) Depth of longitudinal furrow. $(\mu)$

$\begin{array}{rrr}\text { average. } & \max . & \min . \\ 47.58 & 86.50 & 17.30\end{array}$


f) Specific gravity.- 1.413

h) Hardnesss. (kilo)

$\begin{array}{ccc}\text { average. } & \max . & \min . \\ 6.25 & 8.74 & 3.68\end{array}$

i) Volume of $100 \mathrm{~g}$ - 121.0c.c.

j) Weight of 1,000 grains. $-24.2 \mathrm{~g}$.

k) Volume of 1,000 grains.-17.1c.c.

m) Thickness of bran layer. $(\mu)$

$$
\begin{array}{ccc}
\text { average. } & \max . & \min . \\
42.29 & 48.60 & 36.23
\end{array}
$$

p) Chemical composition :-

$\begin{array}{lr}\text { Water } & 12.94 \\ \text { Starch } & 71.40 \\ \text { Sugar } & 1.53 \\ \text { Crude protein } & 9.69 \\ \prime \prime \text { fat } & 2.30 \\ \text { Ash } & 1.34\end{array}$

L) Production of Diye prefecture.

a) Percentage of full grown grains.-95.3

b) External appearance and luster.-Same. as B).

\begin{tabular}{|c|c|c|c|c|c|c|}
\hline \multicolumn{2}{|c|}{ Thickness above } & 2.2 & 76.4 & & 38 & grains \\
\hline '" & '" & 2.0 & 17.8 & " & 9 & "I \\
\hline$" \prime$ & $" r$ & 1.8 & 5.3 & ' & 3 & $\prime \prime$ \\
\hline$" \prime$ & under & 1.8 & 0.6 & $" \prime$ & - & \\
\hline & ngth & $\begin{array}{c}\text { average. } \\
5.30\end{array}$ & $\begin{array}{l}\max . \\
5.70\end{array}$ & & $\begin{array}{l}\min . \\
4.80\end{array}$ & \\
\hline & idth & 3.08 & 3.30 & & 2.85 & \\
\hline
\end{tabular}

c) Size of grains. (m.m.)

d) Percentage of Shinziromai- 19.3

e) Depth of longitudinal furrow. $(x)$

$$
\begin{array}{ccc}
\text { average. } & \max . & \text { min. } \\
45.41 & 09.20 & 25.95
\end{array}
$$

f) Specific gravity:-1.404

h) Hardness. (kilo)

$\begin{array}{ccc}\text { average. } & \max . & \min . \\ 5.22 & 8.51 & 3.38\end{array}$

i) Volume of $100 \mathrm{~g}$.-121.0c.c.

j) Weight of 1,000 grains.-26.2g.

k) Volume of 1,000 grains.-18.5c.c.

m) Thickness of bran layer. $(\mu)$

$$
\begin{array}{rcr}
\text { average. } & \max . & \min . \\
\mathbf{4 5 . 4 1} & 69.20 & \mathbf{2 5 . 9 5}
\end{array}
$$


p) Chemical composition :-

$\begin{array}{lr}\text { Water } & 12.55 \\ \text { Starch } & 73.51 \\ \text { Sugar } & 0.32 \\ \text { Crude protein } & 8.52 \\ \text { " fat } & 2.17 \\ \text { Ash } & 1.06\end{array}$

M) Production of Yelime prefecture.

a) Percentage of full grown grains. $\quad 96.9$

b) External appearance and luster.— same as A).

c) Size of grains. (m.m.)

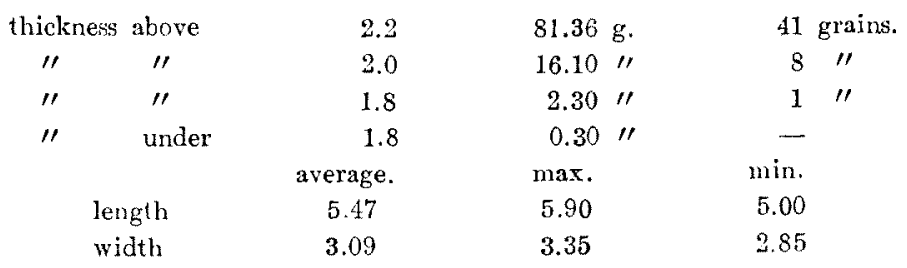

d) Percentage of Shinziromai.—21.8

e) Depth of longitudinal furrow. (k)

$\begin{array}{ccc}\text { average. } & \max . & \min . \\ 48.22 & 77.85 & 25.95\end{array}$

f) Specific gravity.- 1.409

h) Hardness. (kilo)

$\begin{array}{ccc}\text { average. } & \max . & \min . \\ 5.34 & 9.19 & 3.84\end{array}$

i) Volume of $100 \mathrm{~g}$. - 120.0c.c.

j) Weight of 1,000 grains.—27.1g.

k) Volume of 1,000 grains - 19.4c.c.

$\mathrm{m})$ Thickness of bran layer. $(\mu)$

$\begin{array}{rlr}\text { average. } & \max . & \min . \\ 42.12 & 47.70 & \mathbf{3 5 . 3 3}\end{array}$

p) Chemical composition :-

$\begin{array}{lr}\text { Water } & 12.70 \\ \text { Starch } & 71.43 \\ \text { Sugar } & 153 \\ \text { Crude protein } & 9.11 \\ \quad \text { 'fat } & 2.25 \\ \text { Ash } & 1.08\end{array}$

N) Production of Kagawa prefecture.

a) Percentage of full grown grains.- $96.5 \%$

h) External appearance and luster.-Same as M). 
c) Size of grains. (m.m.)

\begin{tabular}{|c|c|c|c|c|c|c|}
\hline thick & above & 2.2 & 88.0 & $g$. & 44 & grains. \\
\hline "I & $\prime \prime$ & 2.0 & 11.0 & $1 \prime$ & 6 & $\prime \prime$ \\
\hline " & $\prime \prime$ & 1.8 & 0.90 & $\prime \prime$ & - & $\prime \prime$ \\
\hline$\prime \prime$ & under & 1.8 & 0.05 & " & - & \\
\hline & & average. & $\max$. & & min. & \\
\hline & gth & 5.44 & 5.85 & & 5.00 & \\
\hline & & 3.11 & 3.40 & & 2.95 & \\
\hline
\end{tabular}

d) Percentage of Shinziromai. -20.2

e) Depth of longitudinal furrow. $(\mu)$

$\begin{array}{ccr}\text { average } & \max . & \min . \\ 38.49 & 60.55 & 17.30\end{array}$

f) Specific gravity.-1.413

h) Hardness. (kilo)

$\begin{array}{ccc}\text { average. } & \max , & \min . \\ 5.98 & 8.74 & \mathbf{3 . 8 4}\end{array}$

i) Volume of $100 \mathrm{~g} .123 .0 \mathrm{c} . \mathrm{c}$.

j) Weight of 1,000 grains. $-27.8 \mathrm{~g}$.

k) Volume of 1,000 grains.-19.8c.c.

m) Thickness of bran layer. $(\mu)$

$\begin{array}{ccc}\text { average. } & \max . & \min . \\ 44.02 & 50.85 & 38.03\end{array}$

p) Chemical composition :-

$\begin{array}{lr}\text { Water } & 12.67 \\ \text { Starch } & 72.88 \\ \text { Sugar } & 1.02 \\ \text { Crude protein } & 8.22 \\ \prime \prime \text { fat } & 2.33 \\ \text { Ash } & 1.08\end{array}$

O) Production of Yelime prefecture.

a) Percentage of full grown grains. - 96.7

b) External appearance and luster:- Same as N).

c) Size of grains. (m.m.)

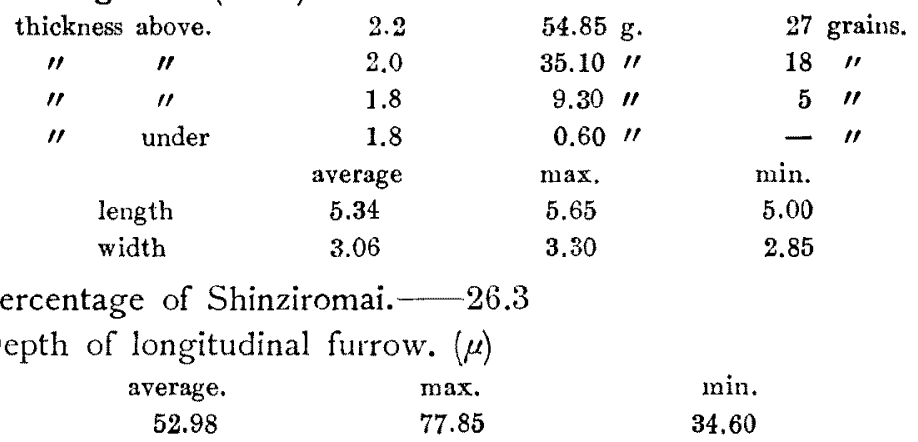


f) Specific gravity. $-\mathbf{1 . 4 1 5}$

h) Hardness. (kilo)

$$
\begin{array}{ccc}
\text { average. } & \max . & \min , \\
5.80 & 9.53 & 3.75
\end{array}
$$

i) Volume of $100 \mathrm{~g}$ - $120.5 \mathrm{c} . \mathrm{c}$.

j) Weight of 1,000 grains. $\quad 25.35 \mathrm{~g}$.

k) Volume of 1,000 grains. - 17.8c.c.

m) Thickness of bran layer. $(\mu)$

$$
\begin{array}{rlr}
\text { average. } & \max . & \min . \\
44.02 & 50.85 & 38.03
\end{array}
$$

p) Chemical composition :-

$\begin{array}{lr}\text { Water } & \mathbf{1 2 . 0 6} \\ \text { Starch } & 72.77 \\ \text { Sugar } & 1.15 \\ \text { Crude protein } & 7.64 \\ \text { " fat } & 2.18 \\ \text { Ash } & 1.16\end{array}$

P) Production of Miyagi prefecture.

a) Percentage of full grown grains. -96.5 luster.

b) External appearance and luster.-Yellowish brown colour, poor of

c) Size of grains. (m.m.)

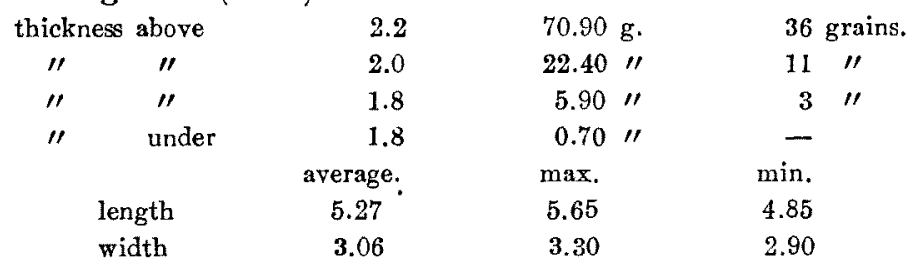

d) Percentage of Shinziromai. -3.6

e) Depth of longitudinai furrow. $(\mu)$

$\begin{array}{ccc}\text { average. } & \max . & \min . \\ 50.17 & 86.50 & 17.30\end{array}$

f) Specific gravity.-1.389

h) Hardness. (kilo)

$$
\begin{array}{ccc}
\text { average. } & \max . & \min . \\
3.61 & 6.34 & 2.57
\end{array}
$$

i) Volume of $100 \mathrm{~g}$. 130.5c.c.

j) Weight of 1,000 grains. $-25.35 \mathrm{~g}$.

k) Volume of 1,000 grains.-18.10c.c.

m) Thickness of bran layer. $(\mu)$

$\begin{array}{ccc}\text { average. } & \max . & \mathrm{min} . \\ 42.68 & 49.50 & 39.60\end{array}$


p) Chemical composition :-

$\begin{array}{lr}\text { Water } & 15.20 \\ \text { Starch } & 73.11 \\ \text { Sugar } & 0.77 \\ \text { Crude protein } & 8.59 \\ \prime \prime \text { fat } & 2.09 \\ \text { Ash } & 1.24\end{array}$

Q) Production of Fukushima prefecture.

a) Percentage of full grown grains. -94.4

b) External appearance and luster- - Nearly same as P).

c) Size of grains. (m.m.)

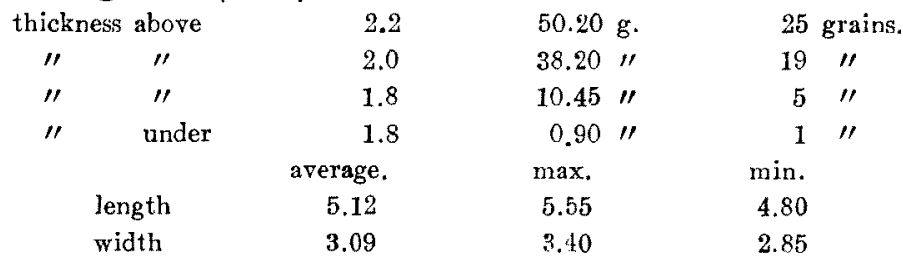

d) Percentage of Shinziromai. -6.6 '

e) Depth of longitudinal furrow. $(\mu)$

$\begin{array}{ccc}\text { average. } & \max . & \min . \\ 55.14 & 86.50 & 17.30\end{array}$

f) Specific gravity. -1.389

h) Hardness. (kilo)

$\begin{array}{ccc}\text { average. } & \max & \min , \\ 3.88 & 6.96 & 2.63\end{array}$

i) Volume of $100 \mathrm{~g} \cdot 133.0 \mathrm{c} . \mathrm{c}$.

j) Weight of 1,000 grains. $\longrightarrow 22.7 \mathrm{~g}$.

k) Volume of 1,000 grains.—16.9c.c.

m) Thickness of bran layer. $(\mu)$

$\begin{array}{ccc}\text { average. } & \max . & \min . \\ 41.56 & 45.00 & 35.78\end{array}$

p) Chemical composition :-

$\begin{array}{lr}\text { Water } & 14.89 \\ \text { Starch } & 73.55 \\ \text { Sugar } & 1.53 \\ \text { Crude protein } & 7.05 \\ \prime \prime \text { fat } & 0.73 \\ \text { Ash } & 0.90\end{array}$

R) Production of Fukushima prefecture.

a) Percentage of full grown grains.- 96.2

b) External appearance and luster.—Same as P). 
c) Size of grains. (m.m.)

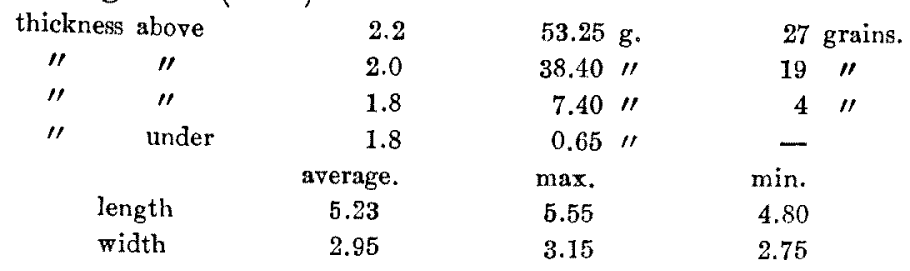

d) Percentage of Shinziromai.-1.8\%

e) Depth of longitudinal furrow. $(\mu)$

$\begin{array}{ccc}\text { average. } & \text { max. } & \min . \\ 47.79 & 69.20 & 17.30 \\ \text { gravity. } & & \\ \text { (kilo) } & & \\ \text { average. } & \max .389 & \min . \\ 4.03 & 5.66 & 2.68\end{array}$

i) Volume of $100 \mathrm{~g}$ - $132.0 \mathrm{c} . \mathrm{c}$.

j) Weight of 1,000 grains. $-23.15 \mathrm{~g}$.

k) Volume of 1,000 grains. 16.4c.c.

$\mathrm{m})$ Thickness of bran layer. $(\mu)$

$\begin{array}{ccc}\text { average. } & \max . & \min . \\ 43.34 & 47.25 & 39.38\end{array}$

p) Chemical composition :-

$\begin{array}{lr}\text { Water } & 14.73 \\ \text { Sarch } & 72.59 \\ \text { Sugar } & 1.35 \\ \text { Crude protein } & 8.37 \\ \quad \prime \prime \text { fat } & 1.01 \\ \text { Ash } & 1.71\end{array}$

S) Production of Fukushima prefecture.

a) Percentage of full grown grains. -90.2

b) External appearance and luster.— Nearly same as $R$ ).

c) Size of grains. (m.m.)

\begin{tabular}{|c|c|c|c|c|c|}
\hline \multicolumn{2}{|c|}{ thickness above } & 2.2 & 79.40 & \multicolumn{2}{|c|}{40 grains. } \\
\hline " & $" \prime$ & 2.0 & 14.10 & 7 & $\prime \prime$ \\
\hline " & " & 1.8 & 5.25 & 3 & $"$ \\
\hline " & under & 1.8 & 2.10 & - & \\
\hline & & average. & $\max$. & $\min$. & \\
\hline & igth & 4,81 & 5.15 & 4.50 & \\
\hline & dth & 3.08 & 3.30 & 2.80 & \\
\hline
\end{tabular}

d) Percentage of Shinziromai.-1.8

e) Depth of longitudinal furrow. $(\mu)$

$\begin{array}{ccc}\text { average. } & \max . & \min . \\ 43.90 & 69.20 & 17.30\end{array}$


f) Specific gravity. $\mathbf{1 . 3 8 9}$

h) Hardness. (kilo)

$\begin{array}{ccc}\text { average. } & \max . & \min . \\ 4.26 & 6.38 & 2.94\end{array}$

i) Volume of $100 \mathrm{~g} .-131.5 \mathrm{c} . \mathrm{c}$.

j) Weight of 1,000 grains. $-23.65 \mathrm{~g}$.

k) Volume of 1,000 grains.—17.0c.c.

m) Thickness of bran layer. $(\mu)$

average. $\max$. min.

$\begin{array}{lll}44.30 & 49.50 & 36.45\end{array}$

p) Chemical composition :-

$\begin{array}{lr}\text { Water } & 15.19 \\ \text { Starch } & 73.51 \\ \text { Sugar } & 0.32 \\ \text { Crude protein } & 8.15 \\ \text { " fat } & 0.60 \\ \text { Ash } & 1.22\end{array}$

B) Polished rice, suitable for the Sake brewing. 19 (A-S) varieties.

A) Production of Okayama prefecture.

b) External appearance and luster.-White glassy luster, grains were fat and large. (1st class).

e) Depth of longitudinal furrow. $(\mu)$

average. $\max$ min.

$\begin{array}{lll}33.30 & 60.55 & 17 \cdot 30\end{array}$

h) Hardness. kilo)

$\begin{array}{ccc}\text { average. } & \max . & \min . \\ 4.17 & 8.06 & 2.63\end{array}$

p) Chemical composition :-

$\begin{array}{lr}\text { Water } & 13.90 \\ \text { Starch } & 76.37 \\ \text { Sugar } & 0.90 \\ \text { Crude protein } & 7.49 \\ \quad \text { " fat } & 0.43 \\ \text { Ash } & 0.39\end{array}$

B) Production of Hyogo prefecture.

b) External appearance and luster.-Nearly same as A).

e) Depth of longitudinal furrow. $(\mu)$
average.
max.
$\min$.
38.28
77.85
25.95

h) Hardness. (kilo)

average.

$\max$ min.

4.02

9.94

2.44 
p) Chemical composition :-

$\begin{array}{lr}\text { Water } & 14.90 \\ \text { Starch } & 73.43 \\ \text { Sugar } & 0.41 \\ \text { Crude protein } & 8.54 \\ \prime \prime \text { fat } & 0.24 \\ \text { Ash } & 0.29\end{array}$

C) Production of Hyogo prefecture.

b) External appearance and luster. Same as B).

e) Depth of longitudinal furrow. $(\mu)$

$\begin{array}{rrr}\text { average. } & \max . & \min . \\ 40.22 & 69.20 & 17.30\end{array}$

h) Hardness. (kile)

$\begin{array}{ccc}\text { average } & \max . & \min . \\ 4.06 & 7.41 & 2.36\end{array}$

p) Chemical composition :-

$\begin{array}{lr}\text { Water } & 13.70 \\ \text { Starch } & 77.53 \\ \text { Sugar } & 0.86 \\ \text { Crude protein } & 8.18 \\ \text { " fat } & 0.13 \\ \text { Ash } & 0.30\end{array}$

D) Production of Osaka prefecture.

b) External appearance and luster.-Same as before.

e) Depth of longitudinal furrow. $(\mu)$

$\begin{array}{ccc}\text { average. } & \max . & \min . \\ 31.57 & 51.90 & 17.30\end{array}$

h) Hardness. (kilo)

average. max. min.

$\begin{array}{lll}4.89 & 7.31 & 2.48\end{array}$

p) Chemical composition :-

$\begin{array}{lr}\text { Water } & 14.70 \\ \text { Starch } & 74.55 \\ \text { Sugar } & 0.41 \\ \text { Crude protein } & 8.18 \\ \text { " fat } & 0.21 \\ \text { Ash } & 0.35\end{array}$

E) Production of Saga prefecture.

b) External appearance and luster.-Nearly same as A).

e) Depth of longitudinal furrow. $(\mu)$
average.
max.
51.90
$\min$.
32.65
17.30

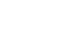


h) Hardness. (kilo)

$\begin{array}{clc}\text { average. } & \max . & \min . \\ 3.92 & 6.38 & 2.36\end{array}$

p) Chemical composition :-

$\begin{array}{lr}\text { Water } & 14.18 \\ \text { Starch } & 72.85 \\ \text { Sugar } & 2.19 \\ \text { Crude protein } & 9.25 \\ \quad \prime \prime \text { fat } & 0.22 \\ \Lambda \text { sh } & 0.42\end{array}$

F) Production of Kumamoto prefecture.

b) External appearance and luster.-Same as E).

e) Depth of longitudinal furrow. $(\mu)$

$\begin{array}{ccc}\text { average. } & \max . & \min . \\ 33.30 & 51.90 & 17.30\end{array}$

h) Hardness. (kilo) average. $\operatorname{man}$. $\min$.

$\begin{array}{lll}3.15 & 5.83 & 2.25\end{array}$

p) Chemical composition :-

$\begin{array}{lr}\text { Water } & 15.30 \\ \text { Starch } & 74.95 \\ \text { Sugar } & 0.81 \\ \text { Crude protein } & 7.32 \\ \prime \prime \text { fat } & 0.20 \\ \text { Ash } & 0.42\end{array}$

G) Production of Hiroshima prefecture.

b) External appearance and luster.—-Nearly same as B).

e) Depth of Jongitudinal furrow. $(\mu)$ average. $\max$. min.

$\begin{array}{lll}24.87 & 43.25 & 0\end{array}$

h) Hardness. (kilo)

average. $\max$ min.

$\begin{array}{lll}4.13 & 7.22 & 2.36\end{array}$

p) Chemical composition :-

$\begin{array}{lr}\text { Water } & 15.40 \\ \text { Starch } & 75.20 \\ \text { Sugar } & 2.19 \\ \text { Crude protein } & 9.25 \\ \prime \prime \prime \text { fat } & 0.20 \\ \text { Ash } & 0.36\end{array}$

H) Production of Okayama prefecture.

b) External appearance and luster.-Same as A).

e) Depth of longitudinal furrow. $(\mu)$ 


$\begin{array}{ccc}\text { average. } & \max . & \min . \\ 30.28 & 51.90 & 17.30\end{array}$

h) Hardness. (kilo)

average

max. $\min$.

$\begin{array}{lll}4.60 & 7.50 & 2.49\end{array}$

p) Chemical composition :-

$\begin{array}{lr}\text { Water } & 15.10 \\ \text { Starch } & 74.97 \\ \text { Sugar } & 1.20 \\ \text { Crude protein } & 9.47 \\ \quad \prime \prime \text { fat } & 0.36 \\ \text { Ash } & 0.42\end{array}$

I) Production of Yamaguchi prefecture.

b) External appearance and luster.- Same as H).

e) Depth of longitudinal furrow. $(\mu)$

$\begin{array}{ccc}\text { average. } & \max . & \min . \\ 33.52 & 51.90 & 17.30\end{array}$

h) Hardness. (kilo)

average. $\max$ min.

p) Chemical composition :-

$\begin{array}{lr}\text { Water } & \mathbf{1 5 . 0 0} \\ \text { Starch } & 76.60 \\ \text { Sugar } & 0.76 \\ \text { Crude protein } & 8.37 \\ \prime \prime \text { fat } & 0.29 \\ \text { Ash } & 0.66\end{array}$

J) Production of Niigata prefecture.

b) External appearance and luster. - Nearly same as D), but slightly brownish colour. (2nd class).

e) Depth of longitudinal furrow. $(\mu)$

$\begin{array}{ccc}\text { average. } & \max . & \min . \\ 30.28 & \mathbf{5 1 . 9 0} & \mathbf{1 7 . 3 0}\end{array}$

h) Hardness. (kilo)

$\begin{array}{ccc}\text { average. } & \max . & \min . \\ 4.89 & 7.31 & 2.48\end{array}$

p) Chemical composition :-

$\begin{array}{lr}\text { Water } & 15.20 \\ \text { Starch } & 76.10 \\ \text { Sugar } & 1.20 \\ \text { Crude protein } & 8.54 \\ \quad \prime \prime \text { fat } & 0.21 \\ \text { Ash } & 0.34\end{array}$


K) Production of Aicli prefecture.

b) External appearance and luster.—_ Same as J).

e) Depth of longitudinal furrow. $(\mu)$

$\begin{array}{ccc}\text { average. } & \max . & \min . \\ 23.57 & 51.90 & 17.30\end{array}$

h) Hardness. (kilo)

$\begin{array}{crr}\text { average. } & \max . & \min . \\ 4.25 & 6.66 & 2.42\end{array}$

p) Chemical composition :-

Water $\quad 14.50$

Starch $\quad 73.94$

Sugar $\quad 2.34$

Crude protein $\quad 7.32$

" fat 0.19

Ash $\quad 0 . \overline{62}$

L) Production of Miye prefecture.

b) External appearance and iuster.—Same as A).

e) Depth of longitudinal furrow. $(\mu)$

average. $\quad$ Inax.

$\begin{array}{lll}15.57 & 25.95 & 0\end{array}$

h) Hardness. (kilo)

average. $\quad \max$. $\min$.

p) Chemical composition :-

Water $\quad \mathbf{1 5 . 3 0}$

Starch $\quad 74.04$

Sugar $\quad 0.71$

Crude protein $\quad 8.18$

" fat 0.13

$\begin{array}{ll}\text { Ash } & 0.57\end{array}$

M) Production of Yelime prefecture.

b) External appearance and luster.-Same as B).

e) Depth of longitudinal furrow. $(\mu)$

$\begin{array}{rcr}\text { average. } & \max . & \min \\ 33.15 & 51.90 & 17.30\end{array}$

h) Hardness. (kilo)

average. max. min.

$\begin{array}{lll}4.09 & 5.72 & 2.59\end{array}$

p) Chemical composition :-

Water $\quad 14.90$

Starch $\quad 75.47$

Sugar $\quad 1.90$

Crude protein $\quad 7.02$ 


$$
\begin{array}{rr}
\prime \prime \text { fat } & 0.24 \\
\text { Ash } & 0.33
\end{array}
$$

N) Production of Kagaya prefecture.

b) External appearance and luster.—Same as B).

e) Depth of longitudinal furrow. $(\mu)$

$\begin{array}{ccc}\text { average. } & \max . & \min . \\ 24.87 & 51.90 & 0\end{array}$

h) Hardness. (kilo).

$\begin{array}{crr}\text { average. } & \max . & \mathrm{min} . \\ 4.70 & 7.50 & 2.36\end{array}$

p) Chemical composition :-

$\begin{array}{lr}\text { Water } & 13.50 \\ \text { Starch } & 77.49 \\ \text { Sugar } & 0.80 \\ \text { Crude protein } & 7.93 \\ \text { " fat } & 0.31 \\ \text { Ash } & 0.34\end{array}$

O) Production of Yehime prefecture.

b) External appearance and luster. Same as N)

e) Depth of longitudinal furrow. $(\mu)$

$\begin{array}{ccc}\text { average. } & \max . & \min . \\ 38.49 & 69.20 & 17.30\end{array}$

h) Hardness. (kilo)

average. $\quad \max$ min.

p) Chemical composition :-

$\begin{array}{lr}\text { Water } & 13.60 \\ \text { Starch } & 73.62 \\ \text { Sugar } & 1.65 \\ \text { Crude protein } & 7.49 \\ \text { " fat } & 0.59 \\ \text { Ash } & 0.43\end{array}$

P) Production of Miyagi prefecture.

b) External appearance and luster.—Nearly same as B).

e) Depth of longitudinal furrow. $(\mu)$

$\begin{array}{ccc}\text { average. } & \max . & \min . \\ 38.06 & 69.20 & 17.30\end{array}$

h) Hardness. (kilo)

$\begin{array}{ccc}\text { average. } & \max . & \min . \\ 2.71 & 5.51 & 1.88\end{array}$

p) Chemical composition :- 


$\begin{array}{lr}\text { Water } & 15.13 \\ \text { Starch } & 75.73 \\ \text { Sugar } & 0.36 \\ \text { Crude protein } & 9.04 \\ \text { " fat } & 0.18 \\ \text { Ash } & 0.31\end{array}$

Q) Production of Fukuslima prefecture.

b) External appearance and luster._- Same as E).

e) Depth of longitudinal furrow. $(\mu)$

$\begin{array}{ccc}\text { average. } & \max . & \min . \\ 45.85 & 69.20 & 17.30\end{array}$

h) Hardness. (kilo)

average. $\quad \max . \quad \min$.

p) Chemical composition :-

$\begin{array}{lr}\text { Water } & 14.80 \\ \text { Starch } & 74.60 \\ \text { Sugar } & 0.36 \\ \text { Crude protein } & 9.04 \\ \prime \prime \text { fat } & 0.14 \\ \text { Ash } & 0.28\end{array}$

R) Production of Fukushima prefecture.

b) External appearance and luster.—Same as Q).

e) Depth of longitudinal furrow. $(\mu)$

$\begin{array}{ccc}\text { average. } & \max . & \min . \\ 38.06 & 51.90 & 17.30\end{array}$

h) Hardness. (kilo)

average. $\quad \max . \quad \min$.

p) Chemical composition :-

$\begin{array}{lr}\text { Water } & 15.60 \\ \text { Starch } & 73.94 \\ \text { Sugar } & 1.10 \\ \text { Crude protein } & 9.47 \\ \text { " fat } & 0.48 \\ \text { Ash } & 0.28\end{array}$

S) Production of Fukushima prefecture.

b) External appearance and luster._- Same as R.

e) Depth of longitudinal furrow. $(\mu)$

$\begin{array}{ccc}\text { average. } & \max . & \min . \\ 35.68 & 77.85 & 0\end{array}$

h) Hardness. (kilo)

$\begin{array}{ccc}\text { average. } & \max . & \min . \\ 3.31 & 5.26 & 1.88\end{array}$


p) Chemical composition :-

$\begin{array}{lr}\text { Water } & 14.57 \\ \text { Starch } & 73.43 \\ \text { Sugar } & 0.41 \\ \text { Crude protein } & 9.04 \\ \prime \prime \text { fat } & 0.27 \\ \text { Ash } & 0.30\end{array}$

$a^{\prime}$ Husked rice, unsuitable for the Saké brewing. $10(\mathrm{I}-\mathrm{X})$ varieties.

I) Production of Fukui prefecture.

a) Percentage of full grown grains. -87.2

b) Exteral appearance and luster.—Straw yellow colour, grains were fat and of middle size, poor of luster. (2nd class)

c) Size of grains. (m.m.)

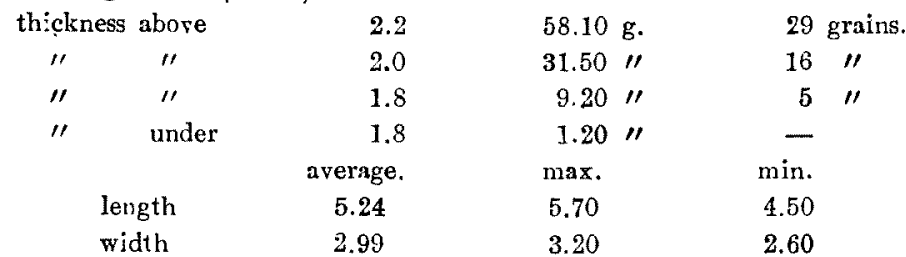

d) Percentage of Shinziromai. -3.0

e) Depth of longitudinal furrow. $(\mu)$

$\begin{array}{ccc}\text { average. } & \max . & \min . \\ 58.17 & 112.45 & 17.30\end{array}$

f) Specific gravity.-1.409

h) Hardness. (kilo)

$\begin{array}{ccc}\text { average. } & \max . & \min . \\ 8.30 & 12.08 & 5.25\end{array}$

i) Volume of $100 \mathrm{~g}$. - 129.0c.c.

j) Weight of 1,000 grains. $-24.7 \mathrm{~g}$.

k) Volume of 1,000 grains. - 17.45c.c.

$\mathrm{m})$ Thickness of bran layer. $(\mu)$

$\begin{array}{ccc}\text { average. } & \max . & \min . \\ 48.29 & 54.45 & 45.00\end{array}$

p) Chemical composition :-

$\begin{array}{lr}\text { Water } & 11.54 \\ \text { Starch } & 72.88 \\ \text { Sugar } & 1.02 \\ \text { Crude protein } & 8.37 \\ \prime \prime \text { fat } & 2.33 \\ \text { Ash } & 1.21\end{array}$

II) Production of Fukui prefecture.

a) Percentage of full grown grains. -90.4 
b) External appearance and luster.-- Same as I).

c) Size of grains. (m.m.)

\begin{tabular}{|c|c|c|c|c|c|}
\hline \multicolumn{2}{|c|}{ thickness above } & 2.2 & $17.50 \mathrm{~g}$. & \multicolumn{2}{|c|}{9 grains. } \\
\hline$\prime \prime$ & $\prime \prime$ & 2.0 & $45.70 \prime \prime$ & 23 & $\prime \prime$ \\
\hline$\prime \prime$ & $\prime \prime$ & 1.8 & $34.50 \prime \prime$ & 17 & $\prime \prime$ \\
\hline " & under & $\begin{array}{c}1.8 \\
\text { average. }\end{array}$ & $\begin{array}{l}2.30 " \prime \\
\max .\end{array}$ & $\begin{array}{c}1 \\
\min .\end{array}$ & $\prime \prime$ \\
\hline & agth & 4.91 & 5.20 & 4.50 & \\
\hline & dth & 2.83 & 3.00 & 2.60 & \\
\hline
\end{tabular}

d) Percentage of Shinziromai.—- $0.6 \%$

e) Depth of longitudinal furrow. (it)

$\begin{array}{ccc}\text { average. } & \max . & \min . \\ 48.01 & 69.20 & 17.30\end{array}$

f) Specific gravity.-1.394

h) Hardness. (kilo)

$\begin{array}{crc}\text { average. } & \max . & \mathrm{min} . \\ 7.96 & 12.11 & 4.56\end{array}$

i) Volume of $100 \mathrm{~g}$. - $126.5 \mathrm{c} . \mathrm{c}$.

j) Weight of 1,000 grains. $-20.05 \mathrm{~g}$.

k) Volume of 1,000 grains.-14.1c.c.

m) Thickness of bran layer. $(\mu)$

average. max. $\min$.

$\begin{array}{lll}44.92 & 48.83 & 38.25\end{array}$

p) Chemical composition :-

$\begin{array}{lr}\text { Water } & 11.78 \\ \text { Starch } & 74.23 \\ \text { Sugar } & 0.77 \\ \text { Crude protein } & 9.25 \\ \prime \prime \text { fat } & 2.29 \\ \text { Ash } & 1.23\end{array}$

III) Production of Saga prefecture.

a) Percentage of full grown grains. -87.5

b) External appearance and luster.__ Same as 1).

c) Size of grains. (m.m.)

\begin{tabular}{ccc} 
thickness above & 2.2 \\
$\prime \prime$ & $\prime \prime$ & 2.0 \\
$\prime \prime$ & $\prime \prime$ & 1.8 \\
$\prime \prime$ & under & 1.8 \\
& \multicolumn{3}{c}{ average. } \\
length & 5.24 \\
width & 3.01 \\
ercentage of Shinziromai.- & 0.8 \\
epth of longitudinal furrow. $(\mu)$ \\
average. \\
44.68 & & max.
\end{tabular}

$\begin{array}{lcc}47.85 \text { g. } & 24 \text { grains. } \\ 36.50 \prime \prime & 18 \prime \prime \\ 13.70 \prime \prime & 7 \prime \prime \\ 1.60 " & 1 " \prime \\ \max . & \text { min. } \\ 5.75 & 4.75 \\ 3.25 & 2.75\end{array}$

d) Percentage of Shinziromai._- 0.8

e) Depth of longitudinal furrow. $(\mu)$
44.68
61.10 
f) Specific gravity. $\mathbf{1} \mathbf{1 . 3 8 9}$

h) Hardness. (kilo)

average.

$\max$.

min.

5.42

7.16

3. 56

i) Volume of $100 \mathrm{~g} . \longrightarrow 131.5 \mathrm{c} . \mathrm{c}$.

j) Weight of 1,000 grains. $-24.40 \mathrm{~g}$.

k) Volume of 1,000 grains._-17.50c.c.

m) Thickness of bran layer. $(\mu)$

$\begin{array}{ccc}\text { average. } & \max . & \min . \\ 48.50 & 51.30 & 45.23\end{array}$

p) Chemical composition :-

$\begin{array}{lr}\text { Water } & 13.90 \\ \text { Stareh } & 73.11 \\ \text { Sugar } & 0.77 \\ \text { Crude protein } & 9.40 \\ \text { " fat } & 0.70 \\ \text { Ash } & 1.21\end{array}$

IV) Production of Hiroshima prefecture.

a) Percentage of full grown grains.-79.4

b) External appearance and luster.— Nearly same as III).

c) Size of grains. (m.m.)

\begin{tabular}{|c|c|c|c|c|c|}
\hline \multicolumn{2}{|c|}{ thickness above } & 2.2 & $52.65 \mathrm{~g}$ & \multicolumn{2}{|c|}{27 grains. } \\
\hline "I & $\prime \prime$ & 2.0 & 30.60 & 15 & $\prime \prime$ \\
\hline " & $\prime \prime$ & 1.8 & 12.60 & 6 & $\prime \prime$ \\
\hline$\prime \prime$ & under & 1.8 & $3.60 \prime$ & 2 & $\prime \prime$ \\
\hline & & average. & $\max$. & min. & \\
\hline & ength & 5.15 & 5.70 & 4.60 & \\
\hline & width & 3.01 & 3.39 & 2.60 & \\
\hline
\end{tabular}

d) Percentage of Shinziromai._- 3.6

e) Depth of longitudinal furrow. $(\mu)$

$\begin{array}{rcr}\text { average. } & \max . & \min . \\ 57.52 & 95.15 & 25.95\end{array}$

f) Specific gravity.- $\mathbf{1 . 4 0 9}$

h) Hardness. (kilo) average. 4.96 $\min$.

3.15

i) Volume of $100 \mathrm{~g}$. 129.0 c.c.

j) Weight of 1,000 grains. $-24.4 \mathrm{~g}$.

k) Volume of 1,000 grains. 17.3c.c.

m) Thickness of bran layer. $(\mu)$

$$
\begin{array}{ccc}
\text { average. } & \max . & \min . \\
45.82 & 49.50 & 39.83
\end{array}
$$

p) Chemical composition :-- 


$\begin{array}{lr}\text { Water } & 13.20 \\ \text { Starch } & 74.23 \\ \text { Sugar } & 0.77 \\ \text { Crude protein } & 8.23 \\ \text { " fat } & 2.09 \\ \text { A } \mathrm{h} & 1.23\end{array}$

V) Production of Yamaguchi frefecture.

a) Percentage of full grown grains.- -87.2

b) External appearance and luster.—-Nearly same as I), but luster was better than $I$.

c) Size of grains. (m.m.)

thickness abore
" $"$
"
" under

2.2

2.0

$50.65 \mathrm{~g}$. $35.65 \mathrm{~m}$

$11.80 \prime \prime$

1.8

length

average.

$1.85 \prime \prime$

$\max$.

5.50

3.20

$\begin{aligned} 25 & \text { grains. } \\ 18 & \prime \prime \\ 6 & \prime \prime \\ 1 & \prime \prime \\ \text { min. } & \\ 4.85 & \\ 2.85 & \end{aligned}$

d) Percentage of Shinziromai._- $3.0 \%$

e) Depth of longitudinal furrow. $(\mu)$

$\begin{array}{ccc}\text { average. } & \max . & \min . \\ 43.03 & 69.20 & 17.30 \\ \text { gravity. } & & \\ \begin{array}{c}\text { (kilo) } \\ \text { average. }\end{array} & & \\ 6.10 & \max . & \min . \\ & 9.15 & 3.94\end{array}$

i) Volume of $100 \mathrm{~g} .-124.0 \mathrm{c} . \mathrm{c}$.

j) Weight of 1,000 grains. $-24.7 \mathrm{~g}$.

k) Volume of 1,000 grains. - 17.5 c.c.

m) Thickness of bran layer. $(\mu)$

$\begin{array}{ccc}\text { average. } & \text { max. } & \min . \\ 47.41 & 51.30 & 43.43\end{array}$

p) Chemical composition :-

$\begin{array}{lr}\text { Water } & 12.82 \\ \text { Starch } & 69.78 \\ \text { Sugar } & 0.71 \\ \text { Crude protein } & 9.40 \\ \prime \prime \text { fat } & 2.31 \\ \text { Ash } & 1.25\end{array}$

VI) Production of Nigat'c prefecture.

a) Percentage of full grown grains._- 93.3

b) External appcarance and luster.- Nearly same as I).

c) Size of grains. (m.m.) 


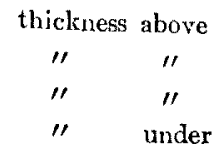

$$
\text { length }
$$$$
\text { width }
$$

2.2
2.0
1.8
1.8
average.
4.77
3.03

$79.70 \mathrm{~g}$.

$15.15 "$

$4.45 \prime \prime$

0.60

$\max$.

5.30

3. 30
40 grains.

8 "

$2 "$

min.

4.25

2.80

d) Percentage of Shinziromai._- 0.7

e) Depth of longitudinal furrow. $(\mu)$

$\begin{array}{ccc}\text { average. } & \max . & \min . \\ 50.82 & 95.15 & 17.30\end{array}$

f) Specific gravity.- -1.385

h) Hardness. (kilo) average.

$\max$.

$\min$.

$$
4.91
$$

6.79

2.70

i) Volume of $100 \mathrm{~g}$._- $-129.0 \mathrm{c.c}$.

j) Weight of 1,000 grains._-23.40g.

k) Volume of 1,000 grains.—16.65c.c.

$\mathrm{m})$ Thickness of bran layer. $(\mu)$

$$
\begin{array}{ccc}
\text { average. } & \text { max. } & \text { min, } \\
49.91 & 54.00 & 46.58
\end{array}
$$

p) Chemical composition :-

$\begin{array}{lr}\text { Water } & 14.01 \\ \text { Starch } & 71.87 \\ \text { Sugar } & 0.90 \\ \text { Crude protein } & 8.23 \\ \text { " fat } & 1.03 \\ \text { Ash } & 1.16\end{array}$

VII) Production of Diye prefecture.

\begin{tabular}{|c|c|c|c|c|c|}
\hline \multicolumn{2}{|c|}{ thickness atove } & 2.2 & $19.90 \mathrm{~g}$. & \multicolumn{2}{|c|}{10 grains. } \\
\hline " & $" \prime$ & 2.0 & $54.50 "$ & 27 & " \\
\hline " & " & 1.8 & $23.00 " \prime$ & 12 & $" \prime$ \\
\hline " & under & $\begin{array}{c}1.8 \\
\text { averaga. }\end{array}$ & $\begin{array}{l}2.50 " 1 \\
\text { max. }\end{array}$ & $\begin{array}{c}1 \\
\text { min. }\end{array}$ & $" 1$ \\
\hline & ggth & 4.94 & 5.80 & 4.65 & \\
\hline & dth & 2.98 & 3.20 & 2.55 & \\
\hline
\end{tabular}

a) Percentage of full grown grains.- 94.4

b) External appearance and luster.-Nearly same as V).

c) Size of grains. (m.m.)

d) Percentage of Shinziromai.-1.1

e) Depth of longitudinal furrow. (k)

$$
\begin{array}{ccc}
\text { average. } & \max . & \min . \\
51.04 & 86.50 & 17.50
\end{array}
$$

f) Specific gravity. $\mathbf{1 . 3 8 9}$ 
h) Hardness. (kilo)

$\begin{array}{ccc}\text { average. } & \max . & \min . \\ 5.61 & 8.33 & 3.28\end{array}$

i) Volume of $100 \mathrm{~g}$.—125.5c.c.

j) Weight of 1,000 grains. $-21.30 \mathrm{~g}$.

k) Volume of 1,000 grains.-14.90c.c.

m) Thickness of bran layer. $(\mu)$

$\begin{array}{ccc}\text { average. } & \max . & \min . \\ 45.17 & 55.13 & 38.70\end{array}$

p) Chemical composition :-

$\begin{array}{lr}\text { Water } & \mathbf{1 2 . 5 3} \\ \text { Starch } & 72.29 \\ \text { Sugar } & 0.32 \\ \text { Crude protein } & 7.93 \\ \text { "fat } & 2.14 \\ \text { Ash } & 1.36\end{array}$

VIII) Production of Kochi prefecture.

a) Percentage of full grown grains._- 81.2

b) External appearance and luster.—-Nearly same as I).

c) Size of grains. (m.m.)

\begin{tabular}{|c|c|c|c|c|c|}
\hline ickr & above & 2.2 & $30.10 \mathrm{~g}$. & $15 \mathrm{~g}$ & ains \\
\hline$" \prime$ & " & 2.0 & $42.10 "$ & 21 & $" \prime$ \\
\hline$"$ & " & 1.8 & $22.30 "$ & 11 & " \\
\hline$" \prime$ & under & 1.8 & 5.30 & 3 & $" 1$ \\
\hline & & average. & $\max$ & $\min$. & \\
\hline & igth & 5.24 & 5.75 & 4.80 & \\
\hline & th & 2.93 & 3.10 & 2.60 & \\
\hline
\end{tabular}

d) Percentage of Shinziromai.-- 1.0

e) Depth of longitudinal furrow. $(\mu)$

$\begin{array}{ccc}\text { average. } & \max . & \min . \\ 60.77 & 103.80 & 17.30\end{array}$

f) Specific gravity.- $1.399 \%$.

h) Hardness. (kilo)

$\begin{array}{ccc}\text { average. } & \max . & \min . \\ 8.32 & 12.45 & 5.01\end{array}$

i) Volume of $100 \mathrm{~g} .-126.0 \mathrm{c} . \mathrm{c}$.

j) Weight of 1,000 grains. $-23.10 \mathrm{~g}$.

k) Volume of 1,000 grains.-16.30c.c.

m) Thickness of bran layer. $(\mu)$

$\begin{array}{ccc}\text { ayerage. } & \max . & \min . \\ 49.50 & 56.25 & 43.88\end{array}$

p) Chemical composition :-- 


$\begin{array}{lr}\text { Water } & 12.70 \\ \text { Starch } & 69.97 \\ \text { Sugar } & \mathbf{1 . 1 5} \\ \text { Crude protein } & 8.52 \\ \text { " fat } & 2.50 \\ \text { Ash } & 1.39\end{array}$

IX) Production of Tokushima prefecture.

a) Percentage of full grown grains.-56.8

b) External appearance and luster.- - Straw brawn colour, destitute of luster, impurities were abundant. (2nd class).

c) Size of grains. (m.m.)

\begin{tabular}{|c|c|c|c|c|c|}
\hline \multicolumn{2}{|c|}{ thickness above } & 2.2 & $41.20 \mathrm{~g}$. & \multicolumn{2}{|c|}{21 grains } \\
\hline$\prime \prime$ & $\prime \prime$ & 2.0 & $30.03 \prime \prime$ & 15 & $\prime \prime$ \\
\hline$\prime \prime$ & $\prime \prime$ & 1.8 & $20.80 \prime \prime$ & 10 & $\prime \prime$ \\
\hline$" \prime$ & under & 1.8 & $7.80 \prime \prime$ & $\begin{array}{r}4 \\
\end{array}$ & $\prime \prime$ \\
\hline & igth & $\begin{array}{c}\text { average. } \\
4.75\end{array}$ & $\begin{array}{c}\max . \\
5.65\end{array}$ & $\begin{array}{r}\min . \\
4.10\end{array}$ & \\
\hline & ath & 2.93 & 3.15 & 2.50 & \\
\hline
\end{tabular}

d) Percentage of Shinziromai.- -0.2

e) Depth of longitudinal furrow. $(\mu)_{\text {. }}$

$\begin{array}{ccc}\text { average. } & \max . & \min . \\ 49.26 & 112.45 & 17.30\end{array}$

f) Specific gravity.- 1.370

h) Hardness. (kilo)

$\begin{array}{crr}\text { average. } & \max . & \min . \\ 5.31 & 7.69 & 3.19\end{array}$

i) Volume of $100 \mathrm{~g} .-127.0$ c.c.

j) Weight of 1,000 grains.-21.1 $\mathrm{g}$.

k) Volume of 1,000 grains.-15.0c.c.

m) Thickness of bran layer. ( $\mu$ )

$\begin{array}{ccc}\text { average. } & \max . & \min . \\ 47.82 & 53.55 & 45.00\end{array}$

p) Chemical composition :-

$\begin{array}{lr}\text { Water } & 13.80 \\ \text { Starch } & 74.63 \\ \text { Sugar } & 1.53 \\ \text { Crude protein } & 8.52 \\ \prime \prime \text { fat } & 2.16 \\ \text { Ash } & 1.23\end{array}$

X) Production of Aomori prefecture.

a) Percentage of full grown grains.-24.7

b) External appearance and luster-Grey brown colour, no luster, impurities were abundant. (3rd class). 
c) Size of grains. (m.m.)

$\begin{array}{cc}\text { thickness above } \\ " \prime & \prime \prime \\ \prime \prime & \prime \prime \\ \prime \prime & \prime\end{array}$

2.2

2.0

$10.70 \mathrm{~g}$

5 grains.

1.8

7.60

$25.20 " \prime$

$46.20 \prime \prime$

length

1.8

average.

width

4.81

$\max$.

5.65

3.15

8 "

13 "

24 "

min.

4.25

2.25

d) Percentage of Shinziromai. -0.3

e) Depth of longitudinal furrow. $(\mu)$

$\begin{array}{ccc}\text { average. } & \max . & \min . \\ 59.90 & 95.15 & 25.95\end{array}$

f) Specific gravity.--

h) Hardness. (kilo)

$\begin{array}{crr}\text { average. } & \max . & \min . \\ 4.38 & 8.16 & 2.44\end{array}$

i) Volume of $100 \mathrm{~g} .-148.5 \mathrm{c} . \mathrm{c}$.

j) Weight of 1,000 grains.

k) Volume of 1,000 grains. -

m) Thickness of bran layer. $(\mu)$ average. $\max$.

min. 51.08

p) Chemical composition :-

$\begin{array}{lr}\text { Water } & 15.18 \\ \text { Starch } & 70.34 \\ \text { Sugar } & 1.35 \\ \text { Crude protein } & 8.81 \\ \text { " fat } & 1.87 \\ \text { Ash } & 1.15\end{array}$

$\beta^{\prime}$ Polished rice, unsuitable for the Saké brewing. 11 (I-XI) varieties.

I) Production of Fukui prefecture.

b) External appearance and luster.-2nd class.

e) Depth of longitudinal furrow. $(\mu)$ average. $\max$.

$39.57 \quad 77.85$

$\min$.

0

h) Hardness. (kilo) average.

5.05

$\max$.

$\min$.

7.01

3.19

p) Chemical composition :-

Water

Starch

72.08

Sugar

0.66 


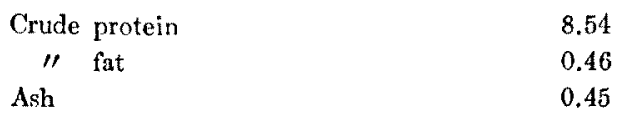

II) Production of Fukui prefecture.

b) External appearance and luster._- Same as I).

e) Depth of longitudinal furrow. $(\mu)$

$\begin{array}{ccc}\text { average. } & \max . & \min . \\ 37.63 & 60.55 & 17.30\end{array}$

h) Hardness. (liilo)

$\begin{array}{cc}\text { average. } \max . & \text { min. } \\ 6.61 & 9.41\end{array}$

p) Chemical composition :-

$\begin{array}{cr}\text { Water } & 14.10 \\ \text { Starch } & 75.68 \\ \text { Sugar } & 0.41 \\ \text { Crude protein } & 8.54 \\ \prime \prime \text { fat } & 0.51 \\ \text { Ash } & 0.46\end{array}$

III) Production of Saga prefecture.

b) External appearance and luster.—Nearly same as I).

e) Depth of longitudinal furrow. $(\mu)$

$\begin{array}{ccc}\text { average. } & \max . & \min . \\ 36.33 & 69.20 & 0\end{array}$

h) Hardness. (kilo)

$\begin{array}{ccc}\text { average. } & \max . & \min . \\ 4.38 & 8.16 & 2.44\end{array}$

p) Chemical composition :-

$\begin{array}{lr}\text { Water } & \mathbf{1 5 . 2 6} \\ \text { Starch } & 72.91 \\ \text { Sugar } & 0.71 \\ \text { Crude protein } & 7.32 \\ \quad \prime \text { fat } & 0.20 \\ \text { Ash } & 0.42\end{array}$

IV) Production of Hiroshima prefecture.

b) External appearance and luster._- Same as I).

e) Depth of longitudinal furrow. $(\ell)$

$\begin{array}{ccc}\text { average. } & \max . & \min . \\ 35.25 & 69.20 & 17.30\end{array}$

h) Hardness. (kilo)

$\begin{array}{ccc}\text { average. } & \max . & \min . \\ 4.54 & 7.78 & 3.02\end{array}$

p) Chemical composition:- 


$\begin{array}{lr}\text { Water } & 14.35 \\ \text { Starch } & 74.55 \\ \text { Sugar } & 1.62 \\ \text { Crude protein } & 8.81 \\ \text { ' fat } & 0.57 \\ \text { Ash } & 0.41\end{array}$

V) Production of Yamaguchi prefecture.

b) Extereal appearance and luster.—Nearly same as IV).

e) Depth of longitudinal furrow. $(\mu)$

average. $\quad \max . \quad \min$,

h) Hardness. (kilo)

$\begin{array}{ccc}\text { average. } & \max . & \min . \\ 6.49 & 11.44 & 3.68\end{array}$

p) Chemical composition :-

$\begin{array}{lr}\text { Water } & 14.30 \\ \mathrm{~S}_{\text {tarch }} & 76.01 \\ \text { Sugar } & 1.42 \\ \text { Crude protein } & 8.54 \\ \text { "f fat } & 0.48 \\ \text { Ash } & 0.56\end{array}$

VI) Production of Nïgata prefecture.

b) External appearance and luster.—Nearly same as I).

e) Depth of longitudinal furrow. $(\mu)$

average. $\max$ min.

h) Hardness. (kilo)

average. $\quad \max . \quad \min$.

p) Chemical composition :-

$\begin{array}{lr}\text { Water } & 14.66 \\ \text { Starch } & 74.24 \\ \text { Sugar } & 0.76 \\ \text { Crude protein } & 9.47 \\ \quad \text { ' fat } & 0.25 \\ \text { Ash } & 0.38\end{array}$

VII) Production of Diye prefecture.

b) External appearance and luster. - Nearly same as V).

c) Depth of longitudinal furrow. $(\mu)$

average. $\quad \max . \quad \min$.

h) Hardness. (kilo)

$\begin{array}{ccc}\text { average.. } & \text { max. } & \min . \\ 2.85 & 4.74 & 1.88\end{array}$


p) Chemical composition :-

$\begin{array}{lr}\text { Water } & 14.90 \\ \text { Starch } & 72.01 \\ \text { Sugar } & 0.71 \\ \text { Crude protein } & 8.18 \\ \text { "fat } & 0.10 \\ \text { Ash } & 0.45\end{array}$

VIII) Production of Kochi prefecture.

b) External appearance and luster.- Somewhat worse than I).

e) Depth of longitudinal furrow. $(\mu)$

$\begin{array}{ccc}\text { average. } & \max . & \min . \\ 4044 & 69.20 & 17.30\end{array}$

h) Hardness. (kilo)

average. $\max$ min.

$\begin{array}{lll}6.61 & 9.41 & 4.35\end{array}$

1) Chemical composition :-

$\begin{array}{lr}\text { Water } & 14.60 \\ \text { Starch } & 73.45 \\ \text { Sugar } & 1.65 \\ \text { Crude protein } & 7.49 \\ \text { " fat } & 0.85 \\ \text { Ash } & 0.38\end{array}$

IX) Production of Tokushima prefecture.

b) External appearance and luster.-Nearly same as I).

e) Depth of longitudinal furrow. $(\mu)$

$\begin{array}{rcr}\text { average. } & \max . & \min . \\ 39.79 & 69.20 & 17.30\end{array}$

h) Hardness. (kilo)

average. $\max$ min.

$\begin{array}{lll}3.37 & 5.93 & 2.12\end{array}$

p) Chemical composition :-

$\begin{array}{lr}\text { Water } & 14.70 \\ \text { Starch } & 72.99 \\ \text { Sugar } & 0.90 \\ \text { Crude protein } & 8.15 \\ \text { " fat } & 0.57 \\ \text { Ash } & 0.99\end{array}$

X) Producion of Aomori prefecture.

b) External appearance and luster. - Nearly same as VIII)

e) Depth of longitudinal furrow. $(\mu)$

$\begin{array}{ccc}\text { average. } & \max . & \min . \\ 50.82 & 86.50 & 17.30\end{array}$

h) Hardness. (kilo) 


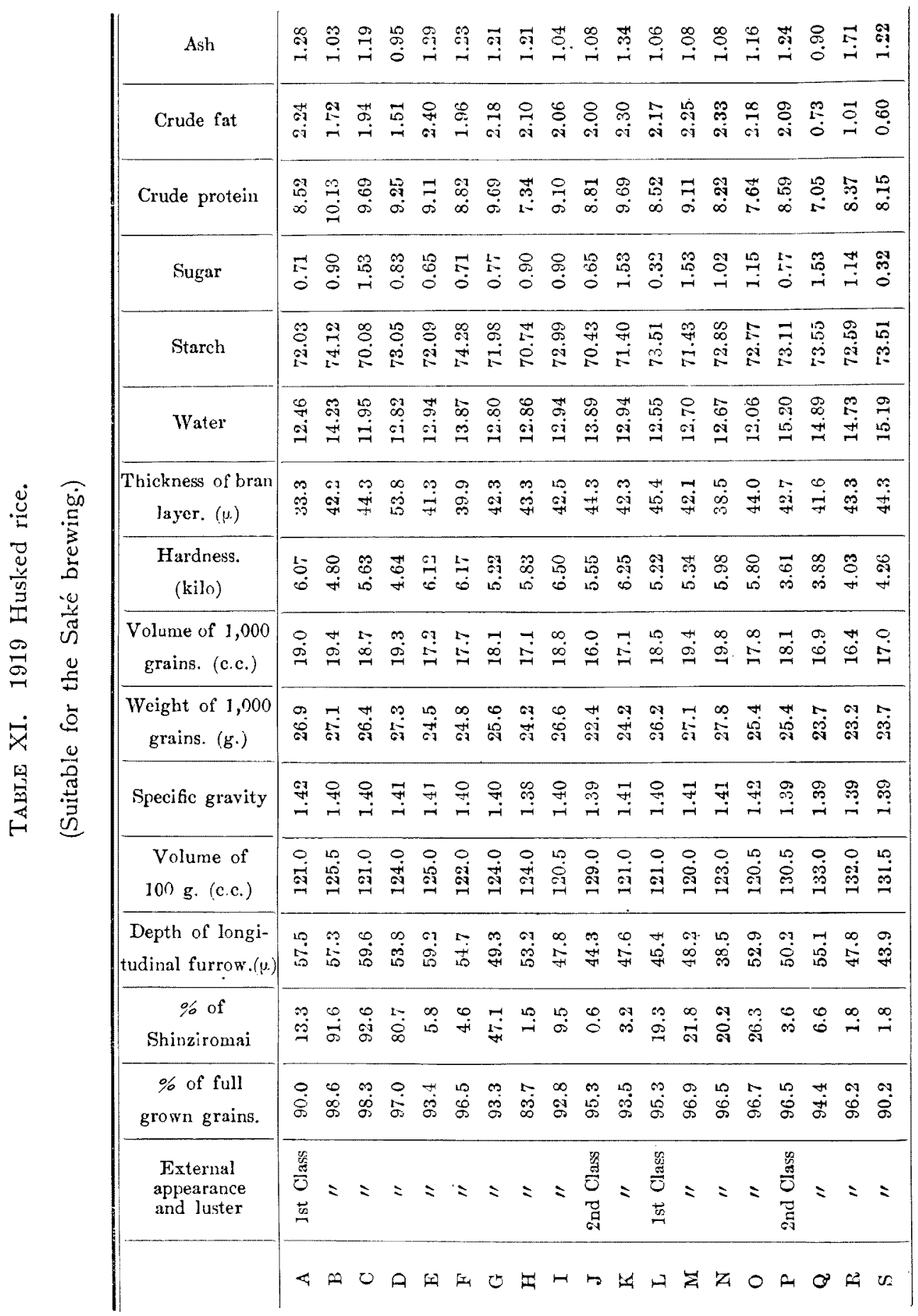




\begin{tabular}{|c|c|c|c|c|c|c|c|c|c|c|c|}
\hline & Ash & $\stackrel{9}{9}$ & 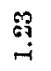 & $\stackrel{\vec{G}}{-1}$ & $\stackrel{9}{\mathscr{B}}$ & & $\stackrel{\oplus}{\stackrel{-}{-1}}$ & 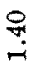 & 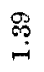 & 舀 & $\stackrel{2}{\underset{7}{-}}$ \\
\hline & Crude fat & $\begin{array}{l}0 \\
\sigma i\end{array}$ & $\begin{array}{l}\text { G] } \\
\text { Gij }\end{array}$ & $\stackrel{8}{0}$ & ${\underset{\sigma i}{0}}_{0}^{\infty}$ & $\begin{array}{l}\vec{\infty} \\
\sigma j\end{array}$ & $\stackrel{\theta}{0}$ & $\underset{6 i}{*}$ & $\begin{array}{l}0 \\
\text { ci } \\
\text { ci }\end{array}$ & 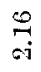 & $\begin{array}{l}\infty \\
- \\
-\end{array}$ \\
\hline & Crude protein & $\begin{array}{l}\infty \\
\infty \\
\infty \\
\infty\end{array}$ & $\begin{array}{l}\text { 品 } \\
\text { o }\end{array}$ & $\begin{array}{l}\text { \&! } \\
\text { هి }\end{array}$ & $\begin{array}{l}9 \\
8 \\
\infty \\
\infty\end{array}$ & $\begin{array}{l}\text { 요 } \\
\text { के }\end{array}$ & \begin{tabular}{l}
$\mathscr{2}$ \\
\hdashline \\
$\infty$ \\
$\infty$
\end{tabular} & $\stackrel{\beta}{2}$ & $\begin{array}{l}\mathscr{9} \\
\infty \\
\infty\end{array}$ & 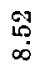 & $\begin{array}{l}\infty \\
\infty\end{array}$ \\
\hline & Sugar & $\stackrel{S}{0}$ & : & 5 & 点 & $\overline{0}$ & $\begin{array}{l}8 \\
:\end{array}$ & $\begin{array}{l}9 \\
\infty \\
0\end{array}$ & $\stackrel{10}{=}$ & $\begin{array}{l}3 \\
-15\end{array}$ & $\stackrel{10}{-0}$ \\
\hline & Starch & $\begin{array}{l}\infty \\
\infty \\
0 j \\
0\end{array}$ & 哭 & @i & की & 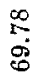 & $\begin{array}{c}\mathfrak{N} \\
-i \\
-1\end{array}$ & $\begin{array}{c}8 \\
i \\
i\end{array}$ & $\begin{array}{l}5 \\
\infty \\
\infty\end{array}$ & $\stackrel{B}{\mathbb{B}}$ & $\stackrel{\leftrightarrow}{0}$ \\
\hline & Water & $\stackrel{-1}{ت}$ & \begin{tabular}{l}
$\infty$ \\
$1:$ \\
\hdashline \\
\hdashline
\end{tabular} & $\begin{array}{l}8 \\
\stackrel{8}{9}\end{array}$ & $\stackrel{8}{8}$ & $\begin{array}{l}6 \\
\infty \\
6 j \\
9 j\end{array}$ & $\underset{0}{0}$ & 䇥 & 足 & $\begin{array}{l}0 \\
\infty \\
\end{array}$ & $\stackrel{\infty}{\infty}$ \\
\hline$\stackrel{\dot{8}}{\dot{E}}$ & $\begin{array}{c}\text { Thickness of bran } \\
\text { layer. (y) }\end{array}$ & $\begin{array}{l}\ddot{0} \\
\dot{\infty} \\
\sharp\end{array}$ & $\underset{\substack{++}}{g}$ & $\begin{array}{l}0 \\
\infty \\
\forall \\
\forall\end{array}$ & 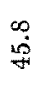 & $\begin{array}{l}* \\
*\end{array}$ & $\stackrel{\sigma}{g}$ & $\overrightarrow{10}$ & بَّ & $\underset{\sim}{\infty}$ & $\stackrel{\vec{B}}{\mathrm{~s}}$ \\
\hline 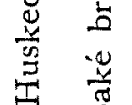 & $\begin{array}{l}\text { Hardness } \\
\text { (kilo) }\end{array}$ & $\begin{array}{l}\stackrel{P}{\infty} \\
\infty \\
\infty\end{array}$ & $\stackrel{\mathscr{\rho}}{\Omega}$ & ספ & $\begin{array}{l}\hat{\sigma} \\
\dot{\forall}\end{array}$ & $\stackrel{0}{\stackrel{9}{6}}$ & $\underset{\dot{\theta}}{\sigma}$ & $\begin{array}{l}-10 \\
0 \\
10\end{array}$ & $\begin{array}{l}\text { 跑 } \\
\infty\end{array}$ & 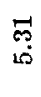 & 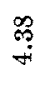 \\
\hline$\underset{\sigma}{\infty} \stackrel{0}{=}$ & $\begin{array}{c}\text { Volume of } 1,000 \\
\text { grains. (c.c.) }\end{array}$ & 点 & $\underset{-}{\stackrel{-}{*}}$ & $\stackrel{0}{\pi}$ & $\stackrel{\leftrightarrow}{\stackrel{\leftrightarrow}{\sim}}$ & $\stackrel{P}{=}$ & $\begin{array}{l}x-1 \\
0\end{array}$ & $\stackrel{\theta}{*}$ & $\stackrel{\oplus}{\oplus}$ & $\underset{10}{0}$ & 1 \\
\hline$\ddot{\infty} \quad \frac{\dot{0}}{0}$ & $\begin{array}{c}\text { Weight of } 1,000 \\
\text { grains. (g.) }\end{array}$ & Ë & $\dot{\dot{8}}$ & $\frac{H}{\Delta}$ & $\underset{\Delta}{\Delta}$ & స્ & $\begin{array}{l}+4 \\
\text { â }\end{array}$ & $\stackrel{\leftrightarrow}{\rightarrow}$ & $\overrightarrow{\mathrm{s}}$ & $\vec{G}$ & 1 \\
\hline 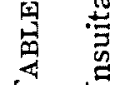 & Specific gravity & $\underset{-}{-}$ & ๓̊ & & $\underset{-}{\sharp}$ & $\stackrel{\infty}{\infty}$ & 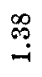 & 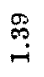 & $\stackrel{9}{\sharp}$ & תִּ & 1 \\
\hline & $\begin{array}{l}\text { Volume of } 100 \mathrm{~g} \text {. } \\
\text { (c.c.) }\end{array}$ & $\stackrel{\circ}{\stackrel{9}{9}}$ & in & $\stackrel{n}{m}$ & $\stackrel{0}{g}$ & $\stackrel{0}{\dot{1}}$ & $\stackrel{9}{9}$ & פת & $\begin{array}{l}0 \\
0 \\
9\end{array}$ & $\stackrel{0}{0}$ & $\begin{array}{l}10 \\
\stackrel{10}{1}\end{array}$ \\
\hline & $\begin{array}{c}\text { Depth of longi- } \\
\text { tudinal furrow. }(\mu)\end{array}$ & $\begin{array}{l}0 \\
\infty \\
\infty \\
10\end{array}$ & 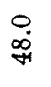 & J & is & $\begin{array}{l}\stackrel{0}{\dot{f}} \\
\text { fin }\end{array}$ & $\begin{array}{l}\infty \\
\dot{8}\end{array}$ & $\stackrel{0}{\text { is }}$ & $\begin{array}{l}\infty \\
\dot{8}\end{array}$ & $\stackrel{m}{\dot{q}}$ & 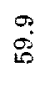 \\
\hline & $\begin{array}{c}\% \text { of } \\
\text { Shinziromai }\end{array}$ & $\underbrace{0}_{0 \rightarrow 0}$ & $\stackrel{0}{\circ}$ & $\stackrel{\infty}{\circ}$ & is & $\underset{\text { oj }}{0}$ & $\ddot{0}$ & 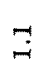 & $\stackrel{\leftrightarrow}{-}$ & $\stackrel{c}{0}$ & $\stackrel{?}{0}$ \\
\hline & $\begin{array}{l}\text { \% of full } \\
\text { grown grains. }\end{array}$ & $\underset{\infty}{\stackrel{9}{0}}$ & $\stackrel{4}{8}$ & $\underbrace{10}_{\infty}$ & $\stackrel{+}{8}$ & $\underset{\infty}{\infty}$ & $\ddot{\leftrightarrow}$ & $\stackrel{\square}{\dot{H}}$ & $\underset{\infty}{\infty}$ & $\begin{array}{l}\infty \\
0 \\
0\end{array}$ & $\vec{H}$ \\
\hline & $\begin{array}{l}\text { External } \\
\text { appearance } \\
\text { and luster }\end{array}$ & 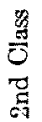 & $=$ & $=$ & $=$ & 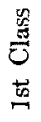 & 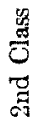 & 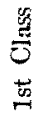 & 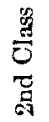 & 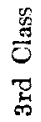 & $=$ \\
\hline & & 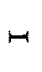 & 咔 & 皀 & $\underline{\nabla}$ & $>$ & $\stackrel{-1}{\longrightarrow}$ & $\stackrel{0}{5}$ & $\underset{\beta}{\vec{\beta}}$ & $\mathcal{A}$ & $x$ \\
\hline
\end{tabular}




\begin{tabular}{|c|c|c|c|c|c|c|c|c|c|c|c|c|}
\hline \multirow{3}{*}{\multicolumn{2}{|c|}{ 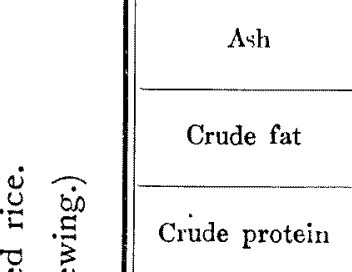 }} & 告 & ti! & $\stackrel{9}{+1}$ & F゙ & $\begin{array}{l}0 \\
0 \\
0 \\
0\end{array}$ & 怘 & 里 & $\begin{array}{c}\infty \\
\stackrel{\infty}{0} \\
\stackrel{0}{0}\end{array}$ & $\stackrel{g}{\stackrel{g}{\circ}}$ & 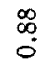 & 总官 \\
\hline & & 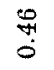 & $\vec{E}$ & శ̊ & t5 & $\stackrel{\infty}{\stackrel{0}{0}}$ & 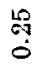 & $\stackrel{9}{0}$ & 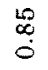 & $\begin{array}{l}5 \\
6 \\
0\end{array}$ & $\begin{array}{l}\circ \\
\stackrel{0}{\circ}\end{array}$ & $\begin{array}{l}\qquad 8 \\
0\end{array}$ \\
\hline & & $\begin{array}{l}\vec{b} \\
\text { 苛 }\end{array}$ & $\begin{array}{l}\not 0 \\
\infty\end{array}$ & 点 & $\begin{array}{c}\bar{\infty} \\
\infty \\
\infty\end{array}$ & $\begin{array}{l}\| \vec{b} \\
\infty\end{array}$ & $\stackrel{\infty}{\stackrel{\infty}{\oplus !}}$ & $\stackrel{\infty}{\infty}$ & $\underset{\stackrel{\sigma}{\sim}}{\stackrel{\sigma}{*}}$ & $\underset{\infty}{\infty}$ & 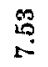 & $\stackrel{g}{\stackrel{g}{S}}$ \\
\hline 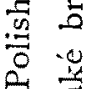 & Starch & 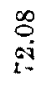 & $\begin{array}{l}\infty \\
0 \\
0 \\
10 \\
10\end{array}$ & $\begin{array}{c}\vec{S} \\
a j\end{array}$ & 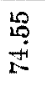 & 家 & 辛 & 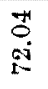 & 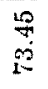 & $\stackrel{8}{8}$ & $\underset{\stackrel{9}{+}}{\stackrel{9}{N}}$ & $\begin{array}{l}\infty \\
\substack{\infty \\
i} \\
0\end{array}$ \\
\hline 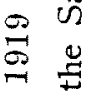 & Water & 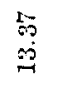 & $\stackrel{\circ}{\dddot{H}}$ & 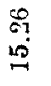 & 望 & $\begin{array}{l}\stackrel{\circ}{9} \\
\stackrel{+}{i}\end{array}$ & $\begin{array}{l}\stackrel{8}{0} \\
\stackrel{+}{*}\end{array}$ & $\begin{array}{l}8 \\
+ \\
+\end{array}$ & 息 & R & $\begin{array}{l}8 \\
\pm \\
\dot{H}\end{array}$ & 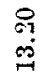 \\
\hline 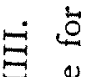 & $\begin{array}{l}\text { Hardness } \\
\text { (kilo) }\end{array}$ & $\begin{array}{l}20 \\
\stackrel{5}{\circ}\end{array}$ & $\begin{array}{l}\overrightarrow{0} \\
\dot{0}\end{array}$ & 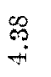 & 薄 & 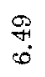 & $\begin{array}{l}\infty \\
\infty \\
\infty \\
\infty\end{array}$ & $\begin{array}{l}\infty \\
\infty \\
o j\end{array}$ & $\begin{array}{l}6 \\
0 \\
0\end{array}$ & 云 & $\begin{array}{l}\infty \\
\infty \\
\infty\end{array}$ & $\stackrel{\vec{t}}{+}$ \\
\hline 茜 & $\begin{array}{l}\text { Depth of longi- } \\
\text { tudinal furrow. ( } \mu\end{array}$ & $\stackrel{\ddot{0}}{\stackrel{0}{0}}$ & 占 & $\ddot{\ddot{\theta}}$ & $\begin{array}{l}07 \\
8 \\
80\end{array}$ & 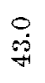 & $\stackrel{5}{4}$ & 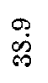 & 范 & $\begin{array}{l}\infty \\
\stackrel{\circ}{\circ}\end{array}$ & $\begin{array}{l}\infty \\
\stackrel{8}{\circ}\end{array}$ & 1 \\
\hline & $\begin{array}{l}\text { External } \\
\text { appearance } \\
\text { and luster }\end{array}$ & 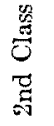 & $=$ & $=$ & $=$ & $=$ & $=$ & 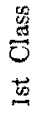 & 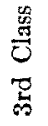 & 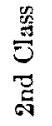 & 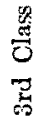 & $=$ \\
\hline & & - & $F$ & $\Xi$ & 5 & $>$ & 5 & $\overrightarrow{5}$ & $\Xi$ & $\Delta$ & $A$ & 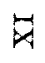 \\
\hline
\end{tabular}

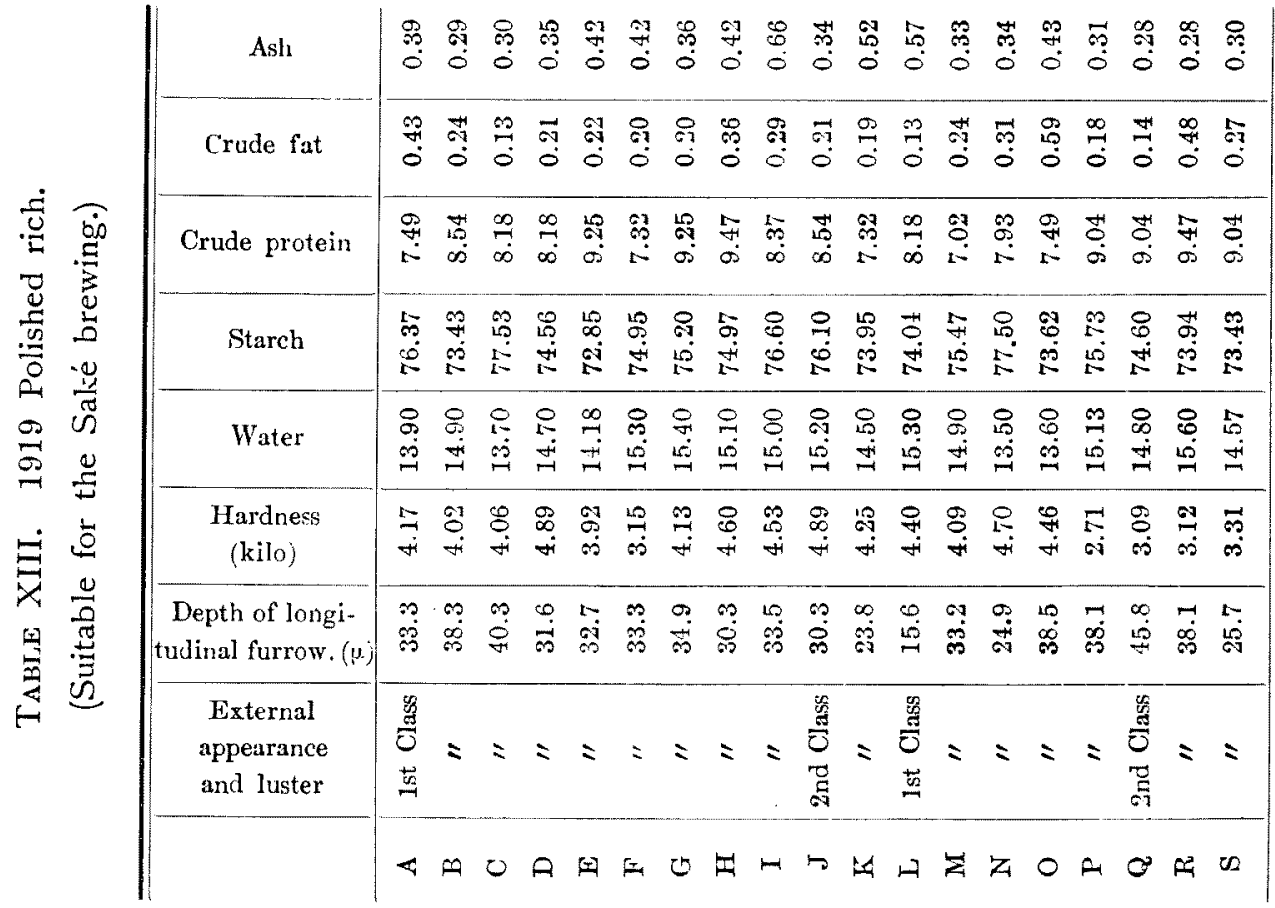




$\begin{array}{ccr}\text { average. } & \max . & \min \\ 3.86 & 7.31 & 2.31 \\ \text { p) Chemical composition :- } & & \\ \text { Water } & & 14.80 \\ \text { Starch } & & 74.12 \\ \text { Sugar } & & 0.90 \\ \text { Crude protein } & & 7.53 \\ \text { " fat } & & 0.69 \\ \text { Ash } & & 0.88\end{array}$

XI) Saigon rice.

b) External appearance and luster.-Nearly same as X), but grains were slender than the former, impurities were abundant.

h) Hardness. (kilo)

$\begin{array}{ccc}\text { average. } & \max . & \min . \\ 4.61 & 6.38 & 3.19\end{array}$

p) Chemical composition :-

$\begin{array}{lr}\text { Water } & 13.20 \\ \text { Starch } & 76.88 \\ \text { Sugar } & 0.32 \\ \text { Crude protein } & 7.93 \\ \text { " fat } & 0.65 \\ \text { Ash } & 0.59\end{array}$

Summary of the 1919 production.

The suitable materials showed high luster and fine external appearance compared with the unsuitable. Percentage of full grown grains, shinziromai, size of grains, specific gravity, both weight and volume of 1,000 grains, and the contents of water, carbohydrates and crude protein were found to be greater in the suitable materials, while depth of longitudinal furrow, volume of $100 \mathrm{~g}$., hardness, thickness of bran layer, crude fat and ash were found to be smaller.

\section{Production.}

a) Husked rice, suitable for the Sake brewing. $23(\mathrm{~A}-\mathrm{W})$ varieties.

A) Production of Okayama prefecture.

a) Percentage of full grown grains. ' 91.4

b) External appearance and luster.- Straw yellow waxy luster, grains were fat and large. (1st class).

c) Size of grains. (m.m.)

$\begin{array}{cc}\text { thickness above } & 2.2 \\ \text { " } & 21 \\ \text { " } & 2.0 \\ \text { " under } & 1.8\end{array}$

$69.5 \mathrm{~g}$.

$25.1 \%$

35 grains. 


$\begin{array}{lccc} & \text { average. } & \text { max. } & \text { min. } \\ \text { length } & \mathbf{5 . 4 2} & \mathbf{5 . 8 5} & 4.85 \\ \text { width } & 3.06 & \mathbf{3 . 2 5} & \mathbf{2 . 9 0}\end{array}$

d) Percentage of Shinziromai.-13.0

e) Depth of longitudinal furrow. $(\mu)$

$\begin{array}{rrr}\text { average. } & \max . & \min . \\ 44.33 & 60.55 & 17.30\end{array}$

f) Specific gravity.-1.418

h) Hardness. (kilo)

$\begin{array}{ccc}\text { average. } & \max \cdot & \min . \\ 5.73 & 8.18 & 3.49\end{array}$

i) Volume of $100 \mathrm{~g} .-124.5 \mathrm{c} . \mathrm{c}$.

j) Weight of 1,000 grains. $-25.75 \mathrm{~g}$.

k) Volume of 1,000 grains.-18.4c.c.

m) Thickness of bran layer. $(\mu)$

$\begin{array}{ccc}\text { average. } & \max . & \min . \\ 41.41 & 44.33 & 37.00\end{array}$

p) Chemical composition :-

$\begin{array}{lr}\text { Water } & 13.64 \\ \text { Starch } & 74.37 \\ \text { Sugar } & 1.99 \\ \text { Crude protein } & 839 \\ \quad \text { "fat } & 1.56 \\ \text { Ash } & 1.22\end{array}$

B) Production of Niigata prefecture.

a) Percentage of full grown grains.-90.6

b) External appearance and luster.-Nearly same as A).

c) Size of grains. (m.m.)

\begin{tabular}{|c|c|c|c|c|c|}
\hline thicknes & above & 2.2 & $64.7 \mathrm{~g}$ & \multicolumn{2}{|c|}{32 grains. } \\
\hline$\prime \prime$ & $\prime \prime$ & 2.0 & $26.6 \prime \prime$ & 13 & $\prime \prime$ \\
\hline " & " & 1.8 & $7.5 \prime \prime$ & 4 & $"$ \\
\hline " & under & 1.8 & 1.0 & 1 & \\
\hline & & average. & $\max$ & $\min$. & \\
\hline & ngth & 5.34 & 5.70 & 4.90 & \\
\hline & idtb & 3.07 & 3.30 & 2.75 & \\
\hline
\end{tabular}

d) Percentage of Shinziromái. 1.6

e) Depth of longitudinal furrow. $(\mu)$ average, $\max$ min. $\begin{array}{lll}48.87 & 69.20 & 25.95\end{array}$

f) Specific gravity.-1.389

h) Hardness. (kilo)

$\begin{array}{ccc}\text { average. } & \max . & \min . \\ 4.36 & 5.48 & \mathbf{3 . 0 4}\end{array}$


i) Volume of $100 \mathrm{~g} .-130.0 \mathrm{c} . \mathrm{c}$.

j) Weight of 1,000 grains. $-24.6 \mathrm{gr}$.

k) Volume of 1,000 grains. $17.6 \mathrm{c} . \mathrm{c}$.

m) Thickness of bran layer. ( $k$ )

$\begin{array}{ccc}\text { average. } & \max . & \min . \\ 44.49 & 50.40 & 38.93\end{array}$

p) Chemical composition :-

38.93

$\begin{array}{lr}\text { Water } & 14.72 \\ \text { Starch } & 74.05 \\ \text { Sugar } & 1.10 \\ \text { Crude protein } & 8.82 \\ \text { " fat } & 0.98 \\ \text { Ash } & 1.28\end{array}$

C) Production of Osaka prefecture.

a) Percentage of full grown grains.-89.4

b) External appearance and luster._- Same as A).

c) Size of grains. (m.m.)

\begin{tabular}{|c|c|c|c|c|c|}
\hline thicknes & s abore & 2.2 & $56.0 \mathrm{~g}$ & 28 & rrains \\
\hline " & $\prime \prime$ & 2.0 & $33.0 \prime \prime$ & 17 & $\prime \prime$ \\
\hline$\prime \prime$ & " & 1.8 & $9.7 \prime \prime$ & 5 & " \\
\hline$\prime \prime$ & under & 1.8 & $1.2 \prime \prime$ & - & \\
\hline & & average. & $\max$. & min. & \\
\hline & ength & 5.42 & 5.75 & 5.00 & \\
\hline & vidth & 3.08 & 3.40 & 3.95 & \\
\hline
\end{tabular}

d) Percentage of Shinziromai.-17.6

e) Depth of longitudinal furrow. $(\mu)$

$\begin{array}{ccc}\text { average. } & \max . & \min . \\ 56.01 & 77.85 & 25.95\end{array}$

f) Specific gravity.- 1.399

h) Hardness. (kilo)

$\begin{array}{crr}\text { average. } & \max . & \mathrm{min} . \\ \mathbf{5 . 1 5} & 7.88 & 3.00\end{array}$

i) Volume of $100 \mathrm{~g} .-125.0 \mathrm{c} . \mathrm{c}$.

j) Weight of 1,000 grains. $25.2 \mathrm{~g}$.

k) Volume of 1,000 grains.-17.9c.c.

m) Thickness of bran layer. $(\mu)$

$\begin{array}{ccc}\text { average. } & \max . & \text { min. } \\ 41.51 & 44.78 & 37.13\end{array}$

p) Chemical composition :-

$\begin{array}{lr}\text { Water } & 14.40 \\ \text { Starch } & 75.48 \\ \text { Sugar } & 0.76 \\ \text { Crude protein } & 8.54 \\ \quad \prime \prime \text { fat } & 1.75 \\ \text { Ash } & 1.17\end{array}$


D) Production of IIyogo prefecture.

a) Percentage of full grown grains.- 89.1

b) External appearance and luster._-Same as C).

c) Size of grains. (m.m.)

\begin{tabular}{|c|c|c|c|c|c|}
\hline thickness & above & 2.2 & $54.8 \mathrm{~g}$. & \multicolumn{2}{|c|}{27 grains } \\
\hline$" \prime$ & $\prime \prime$ & 2.0 & $34.9 \prime \prime$ & 18 & $" \prime$ \\
\hline$"$ & $"$ & 1.8 & 9411 & 5 & $"$ \\
\hline " & under & 1.8 & $0.9 \prime \prime$ & - & \\
\hline & & average. & $\max$ & $\min$. & \\
\hline & igth & 5.55 & 5.85 & 5.15 & \\
\hline & dth & 3.17 & 3.40 & 2.95 & \\
\hline
\end{tabular}

d) Percentage of Shinziromai.—- $80.2 \%$

e) Depth of longitudinal furrow. $(e)$

average. $\max$ mim.

$\begin{array}{lll}60.98 & 86.50 & 25.95\end{array}$

f) Specific gravity.-1.409

h) Hardness. (kilo)

$\begin{array}{ccc}\text { average. } & \max . & \min . \\ 5.59 & 8.61 & 3.19\end{array}$

i) Volume of $100 \mathrm{~g} .-123.0 \mathrm{c} . \mathrm{c}$.

j) Weight of 1,000 grains. $-26.2 \mathrm{~g}$.

k) Volume of 1,000 grains.-18.55c.c.

m) Thickness of bran layer. $(\mu)$

$\begin{array}{ccc}\text { average. } & \max . & \min . \\ 41.05 & 47.48 & 33.75\end{array}$

p) Chemical composition :-

$\begin{array}{lr}\text { Water } & 13.40 \\ \text { Starch } & 75.35 \\ \text { Sugar } & 0.90 \\ \text { Crude protein } & 9.90 \\ \text { " fat } & 1.84 \\ \text { Ash } & 1.24\end{array}$

E) Production of Hyogo prefecture.

a) Percentage of full grown grains.-- 95.1

b) External appearance and luster.-Same as C).

c) Size of grains. (m.m.)

\begin{tabular}{|c|c|c|c|c|c|}
\hline thickness & above & 2.2 & $55.90 \mathrm{~g}$. & 28 & grains. \\
\hline " & $\prime \prime$ & 2.0 & $36.40 " \prime$ & 18 & $\prime \prime$ \\
\hline$\prime \prime$ & $n$ & 1.8 & $7.20 " \prime$ & 4 & $\prime \prime$ \\
\hline$\prime \prime$ & under & 1.8 & $0.50 " \prime$ & - & \\
\hline & length & $\begin{array}{c}\text { average. } \\
5.47\end{array}$ & $\begin{array}{c}\max . \\
5.85\end{array}$ & $\begin{array}{l}\min . \\
5.10\end{array}$ & \\
\hline & width & 3.19 & 3.40 & 3.00 & \\
\hline
\end{tabular}


d) Percentage of Shinziromai.- -79.8

e) Depth of longitudinal furrow. $(\mu)$

$\begin{array}{rl}\text { sverage. } & \max . \\ 56.01 & 86.50\end{array}$

min.

$\begin{array}{lll}56.01 & 86.50 & 25.95\end{array}$

f) Specific gravity. 1.413

h) Hardness. (kilo)

average. max. min.

i) Volume of $100 \mathrm{~g}$ - $121.0 \mathrm{c} . \mathrm{c}$.

j) Weight of 1,000 grains. $-25.5 \mathrm{~g}$.

k) Volume of 1,000 grains.-18.1c.c.

m) Thickness of bran layer. $(\mu)$

$\begin{array}{rrr}\text { average. } & \max . & \min . \\ 41.81 & 46.58 & 37.58\end{array}$

p) Chemical composition :-

$\begin{array}{lr}\text { Water } & 1280 \\ \text { Starch } & 73.19 \\ \text { Sugar } & 1.35 \\ \text { Crude protein } & 7.53 \\ \text { " fat } & 2.07 \\ \text { Ash } & 1.28\end{array}$

F) Production of Hyogo prefecture.

a) Percentage of full grown grains. - 93.1

b) External appearance and luster.- Same as C).

c) Size of grains. (m.m.)

\begin{tabular}{|c|c|c|c|c|c|}
\hline \multicolumn{2}{|c|}{ thickness above } & 2.2 & $58.30 \mathrm{~g}$ & \multicolumn{2}{|c|}{29 grains. } \\
\hline " & $\prime \prime$ & 2.0 & 32.90 & 17 & $" \prime$ \\
\hline "I & $" \prime$ & 1.8 & 8.20 & 4 & " \\
\hline \multirow[t]{4}{*}{ " } & under & 1.8 & 0.50, & - & \\
\hline & & average. & $\max$. & min. & \\
\hline & gth & 5.46 & 5.80 & 5.60 & \\
\hline & th & 3.17 & 3.30 & 2.85 & \\
\hline
\end{tabular}

d) Percentage of Shinziromai.- 72.6

f) Specific gravity.-1.413

h) Hardness. (kilo)

$\begin{array}{ccc}\text { average. } & \max . & \min . \\ 5.00 & 7.41 & 2.85\end{array}$

i) Volume of $100 \mathrm{~g}$. - $124.0 \mathrm{c} . \mathrm{c}$.

j) Weight of 1,000 grains. $-25.9 \mathrm{~g}$.

k) Volume of 1,000 grains._-18.4c.c.

p) Chemical composition :- 


$\begin{array}{cc}\text { Sugar } & 1.25 \\ \text { Crude protein } & 9.47 \\ \prime \prime \text { fat } & 1.35 \\ \text { Ash } & 1.24\end{array}$

G) Production of Okayama prefecture.

a) Percentage of full grown grains._-92.9

b) External appearance and luster.-Same as B).

c) Size of grains. (m.m.) thickness above

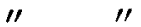

" $" \prime$

under

length

width

2.2

2.0

1.8

1.8 average. 5.46

3.12
$82.70 \mathrm{~g}$

$12.40 " 1$

$3.80 \prime \prime$

$0.90 " \prime$

max.

5.80

335
1.25

1.35

1.24

d) Percentage of Shinziromai.- 79.9

e) Depth of longitudinal furrow. ( $x)$

$\begin{array}{ccr}\text { average. } & \max . & \min . \\ 51.91 & 86.50 & 25.95\end{array}$

f) Shecific gravity.-1.399

h) Hardness. (kilo)

$\begin{array}{ccc}\text { average } & \max . & \text { min. } \\ 5.13 & 7.13 & 3.86\end{array}$

i) Volume of $100 \mathrm{~g} .-124.5 \mathrm{c} . \mathrm{c}$.

j) Weight of 1,000 grains. $-27.2 \mathrm{~g}$.

k) Volume of 1,000 grains.-19.4c.c.

m) Thickness of bran layer. $(\mu)$

average. $\max$. $\min$.

p) Chemical composition :-

$\begin{array}{lr}\text { Water } & 14.40 \\ \text { Starch } & 75.65 \\ \text { Sugar } & 1.20 \\ \text { Crude protein } & 8.82 \\ \quad \prime \prime \text { fat } & 2.14 \\ \text { Ash } & 1.10\end{array}$

H) Production of Okayama prefecture

a) Percentage of full grown grains.- 98.9

b) External appearance and luster.-Same as G).

c) Size of grains. (nı.m.)

\begin{tabular}{|c|c|c|c|c|c|}
\hline thick & bove & 2.2 & $36.7 \mathrm{~g}$ & 18 & grains. \\
\hline$\prime \prime$ & $\prime \prime$ & 2.0 & 41.5 & 21 & $\prime \prime$ \\
\hline "I & $\prime \prime$ & 1.8 & 19.1 & 10 & $\prime \prime$ \\
\hline " & under & 1.8 & 2.7 & 1 & "I \\
\hline
\end{tabular}




$\begin{array}{lccc} & \text { avernge. } & \max . & \min . \\ \text { length } & 5.40 & 5.95 & 5.00 \\ \text { width } & 3.08 & 3.30 & 2.90\end{array}$

d) Percentage of Shinziromai. - 24.0

e) Depth of longitudinal furrow. $(\mu)$

$\begin{array}{ccr}\text { average. } & \max . & \operatorname{min.} \\ 59.04 & 86.50 & 43.25\end{array}$

f) Specific gravity. -1.409

h) Hardness. (kilo)

$\begin{array}{crr}\text { average. } & \max . & \min . \\ 5.72 & 8.55 & 4.24\end{array}$

i) Volume of $100 \mathrm{~g} \cdot-127.5 \mathrm{c} . \mathrm{c}$.

j Weight of 1,000 grains. $\quad 24.60 \mathrm{~g}$.

k) Volume of 1,000 grains. - 17.45c.c.

m) Thickness of bran layer. $(\mu)$

$$
\begin{array}{ccc}
\text { average. } & \max . & \min . \\
41.45 & 44.78 & 38.03
\end{array}
$$

p) Cnemical composition :-

$\begin{array}{lr}\text { Water } & 13.00 \\ \text { Starch } & 72.95 \\ \text { Sugar } & 1.70 \\ \text { Crude prolein } & 7.75 \\ \quad \text { " fat } & 1.87 \\ \text { Ash } & 1.34\end{array}$

I) Production of Okayama Prefecture.

a) Percentage of full grown grains. -92.7

b) External appearance and luster-Same as H).

c) Size of grains. (m.m.)

\begin{tabular}{|c|c|c|c|c|c|}
\hline thick & above & 2.2 & $61.0 \mathrm{~g}$. & 31 & rains \\
\hline$\prime \prime$ & $\prime \prime$ & 2.0 & $31.0^{\prime \prime}$ & 16 & $\prime \prime$ \\
\hline$\prime \prime$ & "f & 1.8 & 7.411 & 3 & 11 \\
\hline$\prime \prime$ & under & 1.8 & $0.6 \prime \prime$ & - & \\
\hline & & average. & $\max$. & min. & \\
\hline & gth & 5.45 & 5.80 & 5.15 & \\
\hline & lth & 3.07 & 3.25 & 2.85 & \\
\hline
\end{tabular}

d) Percentage of Shinziromai.-72.8

e) Depth of longitudinal furrow $(\mu)$

$\begin{array}{ccc}\text { average. } & \max . & \min . \\ 51.68 & 86.50 & 34.60\end{array}$

f) Specific gravity.- 1.404

h) Hardness (kilo)

$\begin{array}{clr}\text { average. } & \text { max. } & \text { min. } \\ 6.16 & 9.49 & 3.86\end{array}$


i) Volume of $100 \mathrm{~g}$ - $123.0 \mathrm{c} . \mathrm{c}$.

j) Weight of 1,000 grains. $-25.85 \mathrm{~g}$.

k) Volume of 1,000 grains.-18.4c.c.

m) Thickness of bran layer. $(\mu)$

$\begin{array}{ccc}\text { average. } & \max . & \min . \\ 43.55 & 47.25 & 38.70\end{array}$

p) Chemical composition :-

$\begin{array}{lr}\text { Water } & 12.60 \\ \text { Starch } & 75.35 \\ \text { Sugur } & 0.90 \\ \text { Crude protein } & 9.04 \\ \text { " fat } & 2.43 \\ \text { Ash } & 1.27\end{array}$

J) Production of Hiroshima prefecture.

a) Percentage of full grown grains.-94.8

b) External appearance and luster.-Same as $\mathbf{f}$ ).

c) Size of grains. (m.m.)

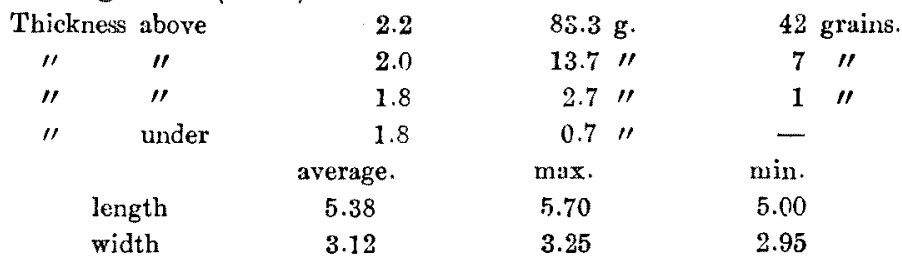

d) Percentage of Shinziromai. -66.0

e) Depth of longitudinal furrow. $(\mu)$

$\begin{array}{ccc}\text { average. } & \max . & \min . \\ 50.60 & 69.20 & 34.60\end{array}$

f) Specific gravity.-1.409

h) Hardness. (kilo)

$\begin{array}{clc}\text { average. } & \max . & \min . \\ 5.46 & 9.75 & 3.56\end{array}$

i) Volume of $100 \mathrm{~g}$ - 125.0 c.c.

j) Weight of 1,000 grains. $-26.90 \mathrm{~g}$.

k) Volume of 1,000 grains._-19.10c.c.

m) Thickness of bran layer. $(\mu)$

$\begin{array}{ccc}\text { average. } & \text { max. } & \text { min. } \\ 41.45 & 44.58 & 38.03\end{array}$

p) Chemical composition :-

$\begin{array}{lr}\text { Water } & 12.80 \\ \text { Starch } & 74.90 \\ \text { Sugar } & 1.40 \\ \text { Crude protein } & 9.47 \\ \quad \prime \prime \text { fat } & 1.99 \\ \text { Ash } & 1.17\end{array}$


K) Production of Okayama prefccture.

a) Percentage of full grown grains.- 90.5

b) External appearance and luster. Same as J).

c) Size of grains. (m.m.)

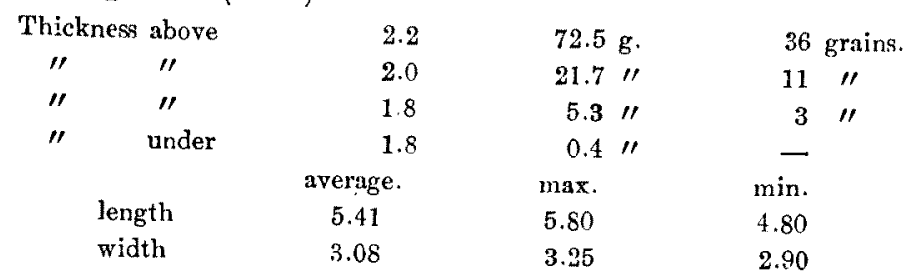

d) Percentage of Shinziromai.-14.8

e) Depth of longitudinal furrow. $(\mu)$

$\begin{array}{ccc}\text { average. } & \max . & \min . \\ 53.41 & 86.50 & 34.60\end{array}$

f) Specific gravity.- -1.404

h) Hardness. (kilo)

$\begin{array}{crc}\text { average. } & \max . & \min \\ 5.55 & 9.00 & 3.64\end{array}$

i) Volume of $100 \mathrm{~g}$ - 126.0c.c.

j) Weight of 1,000 grains $-26.45 \mathrm{~g}$.

k) Volume of 1,000 grains.-18.8c.c.

m) Thickness of bran layer. $(\mu)$

$\begin{array}{crr}\text { average. } & \max . & \min . \\ 41.03 & 44.78 & 37.58\end{array}$

p) Chemical composition :-

$\begin{array}{lr}\text { Water } & 13.80 \\ \text { Starch } & 74.14 \\ \text { Sugar } & 1.00 \\ \text { Crude protein } & 9.90 \\ \text { " fat } & 1.68 \\ \text { Ash } & 1.14\end{array}$

L) Production of Okayama prefecture.
a) Perentage of full grown grains. -93.2
b) External appearance and luster.-Same as J).
c) Size of grains. (m.m.)

\begin{tabular}{|c|c|c|c|c|c|}
\hline \multicolumn{2}{|c|}{ thickness above } & 2.2 & $30.0 \mathrm{~g}$. & \multicolumn{2}{|c|}{15 grains. } \\
\hline " & $" \prime$ & 2.0 & $54.0 \prime \prime$ & 27 & $" 1$ \\
\hline " & $"$ & 1.8 & $15.2 " \prime$ & 8 & " \\
\hline " & under & 1.8 & $0.8 \prime \prime$ & - & $\prime \prime$ \\
\hline & gth & $\begin{array}{c}\text { average. } \\
5.26\end{array}$ & max. & $\begin{array}{l}\min . \\
4.85\end{array}$ & \\
\hline & & 3.03 & 3.20 & 2.85 & \\
\hline
\end{tabular}


d) Percentage of Shinziromai. -3.4

e) Depth of longitudinal furrow. ( $/$ )

$\begin{array}{ccc}\text { arerage. } & \text { max. } & \text { min. } \\ 53.85 & 86.50 & 17.30\end{array}$

f) Specific gravity. -1.418

h) Hardness. (kilo)

$\begin{array}{ccc}\text { average. } & \max . & \min . \\ 5.62 & 8.00 & \mathbf{3 . 6 6}\end{array}$

i) Volume of $100 \mathrm{~g}$ - 125.0 c.c.

j) Weight of 1,000 grains. $-23.9 \mathrm{~g}$.

k) Volume of 1,000 grains.-16.9c.c.

$\mathrm{m})$ Thickness of bran layer. $(\mu)$

$\begin{array}{ccr}\text { average. } & \max . & \min . \\ 41.98 & 47.70 & 38.25\end{array}$

p) Chemical composition :-

$\begin{array}{lr}\text { Water } & 13.00 \\ \text { Starch } & 73.18 \\ \text { Sugar } & 0.81 \\ \text { Crude protein } & 9.47 \\ \prime \prime \text { fat } & 2.01 \\ \text { Ash } & 1.31\end{array}$

M) Production of Okayama prefecture.

a) Percentage of full grown grains. 97.5

b) External appearance and luster-_-Same as B).

c) Size of grains. (m.m.)

\begin{tabular}{|c|c|c|c|c|c|c|}
\hline \multicolumn{2}{|c|}{ thickness above } & \multirow{2}{*}{$\begin{array}{l}2.2 \\
2.0\end{array}$} & \multirow{2}{*}{$\begin{array}{l}63.8 \\
29.9\end{array}$} & & \multicolumn{2}{|c|}{32 grains. } \\
\hline "I & $\prime \prime$ & & & $\prime \prime$ & 15 & $\prime \prime$ \\
\hline "I & $\prime \prime$ & 1.8 & 5.8 & $\prime \prime$ & 3 & $\prime \prime$ \\
\hline \multirow[t]{4}{*}{$\prime \prime$} & under & 1.8 & 0.4 & $\prime \prime$ & - & \\
\hline & & average & $\max$. & & $\min$. & \\
\hline & & 5.48 & 5.80 & & 5.0 & \\
\hline & & 3.13 & 3.35 & & 3.0 & \\
\hline
\end{tabular}

d) Percentage of Shinziromai. -83.0

e) Depth of longitudinal furrow. $(\mu)$

$\begin{array}{ccc}\text { average. } & \max . & \min . \\ 54.49 & 86.50 & 17.30\end{array}$

f) Specific gravity. -1.409

h) Hardness. (kilo)

$\begin{array}{ccc}\text { average. } & \max . & \min . \\ 5.47 & 7.22 & \mathbf{2 . 9 3}\end{array}$

i) Volume of $100 \mathrm{~g} .-122.0 \mathrm{c} . \mathrm{c}$.

j) Weight of 1,000 grains. $-25.7 \mathrm{~g}$.

k) Volume of 1,000 grains.-18.35c.c. 
m) Thickness of bran layer. $(\mu)$

$$
\begin{array}{ccr}
\text { average. } & \max . & \min , \\
41.28 & 44.78 & \mathbf{3 6 . 6 8}
\end{array}
$$

p) Chemical composition :-

$\begin{array}{lr}\text { Water } & 14.40 \\ \text { Starch } & 73.79 \\ \text { Sugar } & 0.76 \\ \text { Crude protein } & 9.90 \\ \text { " fat } & 1.90 \\ \text { Ash } & 1.22\end{array}$

N) Production of Okayama prefecture.
a) Percentage of full grown grains. -88.85
b) External appearance and luster. Same as M).

\begin{tabular}{|c|c|c|c|c|c|}
\hline \multicolumn{2}{|c|}{ thickness above } & 2.2 & $85.0 \mathrm{~g}$ & \multicolumn{2}{|c|}{43 grains } \\
\hline$\prime \prime$ & $\prime \prime$ & 2.0 & $12.1 "$ & 6 & $" \prime$ \\
\hline " & $" \prime$ & 1.8 & $2.5 \prime$ & 1 & " \\
\hline 11 & under & 1.8 & $0.3 \prime$ & - & \\
\hline & & average. & $\max$ & $\min$. & \\
\hline & gth & 5.51 & $5: 90$ & 5.15 & \\
\hline & & 3.19 & 3.40 & 3.00 & \\
\hline
\end{tabular}
c) Size of grains. (m.m.)

d) Percentage of Shinziromai.-25.2

\begin{tabular}{|c|c|c|}
\hline $\begin{array}{r}\text { average. } \\
49.52\end{array}$ & $\begin{array}{l}\max . \\
69.20\end{array}$ & $\begin{array}{l}\min . \\
34.60\end{array}$ \\
\hline $\begin{array}{l}\text { ravity. } \\
\text { (kilo) }\end{array}$ & & \\
\hline average. & max. & min. \\
\hline
\end{tabular}

e) Depth of longitudinal furrow. $(\mu)$

i) Volume of $100 \mathrm{~g}$ - 126.0 c.c.

j) Weight of 1,000 grains. $-27.7 \mathrm{~g}$.

k) Volume of 1,000 grains.-19.75c.c.

m) Thickness of bran layer. $(\mu)$

$\begin{array}{crr}\text { average. } & \max . & \min . \\ 41.70 & 46.80 & 38.70\end{array}$

p) Chemical composition :-

$\begin{array}{lr}\text { Water } & 14.07 \\ \text { Starch } & 72.79 \\ \text { Sugar } & 1.70 \\ \text { Crude protein } & 9.90 \\ \text { " fat } & 1.52 \\ \text { Ash } & 1.13\end{array}$

O) Production of Kumamoto prefecture. 
a) Percentage of full grown grains.-91.4

b) External appearance and Iuster.-Nearly same as B).

c) Size of grains. (m.m.)

\begin{tabular}{ccc} 
thickness above & 2.2 \\
$\prime \prime$ & $\prime \prime$ & 2.0 \\
$\prime \prime$ & $\prime \prime$ & 1.8 \\
$\prime \prime$ & under & 1.8 \\
& \multicolumn{3}{c}{ average. } \\
& length & 5.07 \\
& width & 2.98 \\
Percentage of Shinziromai. -1.4 \\
Pepth of longitudinal furrow. $(\mu)$
\end{tabular}

$\begin{array}{rrr}51.2 \mathrm{~g} . & 26 \text { grains } \\ 40.2 \prime \prime & 20 \prime \prime \\ 7.8 \prime \prime & 4 \quad \prime \\ 0.8 \prime \prime & - \\ \max . & \min . \\ 5.75 & 4.75 \\ 3.20 & 2.65\end{array}$

d) Percentage of Shinziromai.-1.4

e) Depth of longitudinal furrow. $(\mu)$

$\begin{array}{ccc}\text { average. } & \max . & \min . \\ 58.39 & 103.80 & \mathbf{3 4 . 6 0}\end{array}$

f) Specific gravity. -1.394

h) Hardness. (kilo)

$\begin{array}{ccc}\text { average. } & \max . & \min . \\ 5.13 & 7.54 & 3.49\end{array}$

i) Volume of $100 \mathrm{~g}$. $129.0 \mathrm{c} . \mathrm{c}$.

j) Weight of 1,000 grains. $-24.13 \mathrm{~g}$.

k) Volume of 1,000 grains.-17.20c.c.

m) Thickness of bran layer. $(k)$

$\begin{array}{ccc}\text { average. } & \max . & \min . \\ 42.99 & 47.70 & 39.83\end{array}$

p) Chemical composition :-

Water $\quad 15.20$

Starch $\quad 75.31$

Sugar $\quad 0.95$

Crude protein $\quad 9.47$

"fat 1.74

Ash $\quad 1.22$

P) Production of Kumamoto prefecture.

a) Percentage of full grown grains. -82.2

b) External appearance and luster.-Same as M).

c) Size of grains. (m.m.)

\begin{tabular}{|c|c|c|c|c|c|c|}
\hline thick & above & 2.2 & 54.7 & & 27 & grains. \\
\hline$\prime \prime$ & $\prime \prime$ & 2.0 & 34.4 & $" \prime$ & 17 & $" \prime$ \\
\hline " & " & 1.8 & 9.6 & $\prime \prime$ & 5 & $\prime \prime$ \\
\hline$\prime \prime$ & under & 1.8 & 1.2 & $\prime \prime$ & 1 & $" \prime$ \\
\hline & & average. & $\max$. & & $\min$. & \\
\hline & gth & 5.18 & 5.65 & & 4.75 & \\
\hline & dth & 3.07 & 3.30 & & 2.70 & \\
\hline
\end{tabular}

d) Percentage of Shinziromai. -9.1

e) Depth of longitudinal furrow. (v) 


\begin{tabular}{|c|c|c|}
\hline $\begin{array}{c}\text { average. } \\
56.87\end{array}$ & $\begin{array}{l}\max . \\
77.75\end{array}$ & $\begin{array}{r}\min \\
36.60\end{array}$ \\
\hline $\begin{array}{l}\text { gravity.- } \\
\text { s. (kilo) }\end{array}$ & & \\
\hline arerage. & $\max$. & min. \\
\hline 5.71 & 9.23 & 3.84 \\
\hline
\end{tabular}

i) Volume of $100 \mathrm{~g} .-126.0 \mathrm{c} . \mathrm{c}$.

3.84

j) Weight of 1,000 grains. $-24.3 \mathrm{~g}$.

k) Volume of 1,000 grains. 17.3c.c.

m) Thickness of bran layer. $(\mu)$

$\begin{array}{lrr}\text { average. } & \text { max. } & \min \\ 42.53 & 45.68 & 37.35 \\ \text { composition :- } & & \\ \text { Water } & & 13.60 \\ \text { Starch } & & 72.47 \\ \text { Sugar } & 1.60 \\ \text { Crude protein } & 8.18 \\ \text { "f fat } & 1.39 \\ \text { Ash } & & 1.23\end{array}$

Q) Production of Kumamoto prefecture.

a) Percentage of full grown grains. - 91.3

b) External appearance and luster.—Same as A).

c) Size of grains. (m.m.)

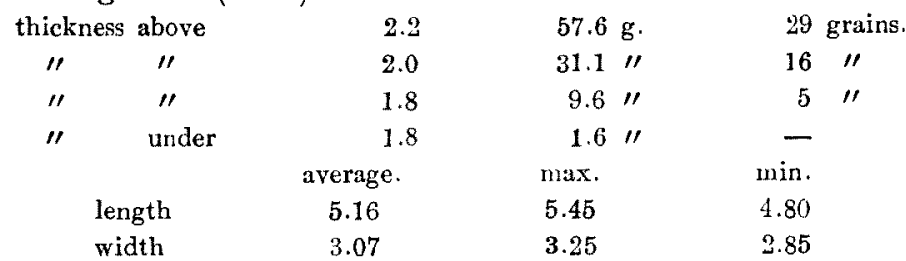

d) Percentage of Shinziromai.- 4.0

e) Depth of longitudinal furrow. $(\mu)$

$\begin{array}{ccc}\text { average. } & \max . & \min . \\ 53.85 & 77.75 & 25.95\end{array}$

f) Specific gravity. 1.399

h) Hardness. (kilo)

$\begin{array}{ccc}\text { average. } & \max . & \min . \\ 5.49 & 7.88 & 3.41\end{array}$

i) Volume of $100 \mathrm{~g} \cdot-126.0 \mathrm{c} . \mathrm{c}$.

j) Weight of 1,000 grains. $--24.2 \mathrm{~g}$.

k) Volume of 1,000 grains. - 17.3c.c.

m) Thickness of bran layer. $(\mu)$

$\begin{array}{ccc}\text { average. } & \max . & \min . \\ 42.13 & 45.23 & 39.15\end{array}$


p) Chemical composition :-

$\begin{array}{lr}\text { Water } & 15.03 \\ \text { Sterch } & 73.80 \\ \text { Sugar } & 1.25 \\ \text { Crude protein } & 9.04 \\ \text { " fat } & 1.54 \\ \text { Ash } & 1.08\end{array}$

R) Production of Kumamoto prefecture.

a) Percentage of full grown grains. $\quad 91.4$

b) External appearance and luster._Same as A).

c) Size of grains (m.m.)

\begin{tabular}{|c|c|c|c|c|c|c|}
\hline \multicolumn{2}{|c|}{ thickness above } & 2.2 & 60.6 & & 30 & grains. \\
\hline$\prime \prime$ & $\prime \prime$ & 2.0 & 30.4 & $\prime \prime$ & 15 & $" \prime$ \\
\hline 11 & $\prime \prime$ & 1.8 & 8.0 & "I & 4 & 11 \\
\hline$\prime \prime$ & under & 1.8 & 1.0 & $\prime \prime$ & 1 & $\prime \prime$. \\
\hline & $a^{\text {th }}$ & $\begin{array}{c}\text { average. } \\
5.13\end{array}$ & $\begin{array}{c}\max . \\
5.75\end{array}$ & & $\begin{array}{r}\min . \\
4.75\end{array}$ & \\
\hline & & 3.01 & 3.30 & & 2.60 & \\
\hline
\end{tabular}

d) Percentage of Shinziromai. 6.6

e) Depth of longitudinal furrow. $(\mu)$

$\begin{array}{ccc}\text { average. } & \text { max. } & \text { min. } \\ 51.47 & 86.50 & 17.30\end{array}$

f) Specific gravity:- 1.404

h) Hardness. (kilo)

$\begin{array}{crr}\text { average. } & \max . & \min . \\ 5.73 & 8.63 & 3.26\end{array}$

i) Volume of $100 \mathrm{~g}$ - 123.0c.c.

j) Weight of 1,000 grains. $-23.75 \mathrm{~g}$.

k) Volume of 1,000 grains.—16.85c.c.

m) Thickness of bran layer. $(\mu)$

$\begin{array}{ccc}\text { average. } & \text { max. } & \text { min. } \\ 43.44 & 48.83 & 39.60\end{array}$

p) Chemical composition :-

$\begin{array}{lr}\text { Water } & 13.30 \\ \text { Starch } & 73.13 \\ \text { Sugar } & 1.50 \\ \text { Crude protein } & 8.54 \\ \quad \prime \text { fat } & 1.84 \\ \text { Ash } & 1.18\end{array}$

S) Production of Akita prefecture.
a) Percentage of full grown grains.-95.3
b) External appearance and luster.—Same as N).
c) Size of grains. (m.m.) 


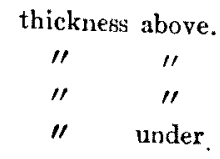

length

width

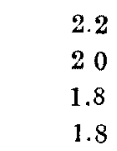

average.

5.20

3.09
$83.0 \mathrm{~g}$.

$14.0 " 1$

$2.7 \prime$

$0.3 \prime$

$\max$.

.5 .55

3.30

d) Percentage of Shinziromai.- 4.4 average.

max.

47.58

77.85

f) Specific gravity. -1.413

h) Hardness. (kilo)

average.

6.30

$\max$.

10.50

i) Volume of $100 \mathrm{~g} . \longrightarrow 126.0 \mathrm{c} . \mathrm{c}$.

j) Weight of 1,000 grains. $-25.1 \mathrm{~g}$.

k) Volume of 1,000 grains. - 17.8c.c.

m) Thickness of bran layer. $(\mu)$ average.

43.62

$\max$.

47.25

min. 40.28

p) Chemical composition :-

$\begin{array}{lr}\text { Water } & 13.90 \\ \text { Starch } & 73.79 \\ \text { Sugar } & 0.76 \\ \text { Crude protein } & 9.90 \\ \quad \text { "fat } & 1.90 \\ \text { Ash } & 1.22\end{array}$

T) Production of Akita prefecture.

a) Percentage of full grown grains.-97.4

b) External appearance and luster.- Same as T).

c) Size of grains. (m.m.)

\begin{tabular}{|c|c|c|}
\hline \multicolumn{2}{|c|}{ thickness above } & 2.2 \\
\hline$" \prime$ & $" \prime$ & 2.0 \\
\hline$" \prime$ & " & 1.8 \\
\hline$\prime \prime$ & under & 1.8 \\
\hline & & average. \\
\hline & gth & 5.14 \\
\hline
\end{tabular}

$80.0 \mathrm{~g}$

$18.2 \%$

$1.6 \prime \prime$

$0.1 " 1$

max.

5.65

3.30

min.

3.19

min.

25.95

min.

4.95

3.00
42 grains.

7 "

11

- 11

5 
h) Hardness. (kilo)

$\begin{array}{crr}\text { average. } & \max . & \min . \\ 4.61 & 7.58 & 2.89\end{array}$

i) Volume of $100 \mathrm{~g}$ - - $125.5 \mathrm{c} . \mathrm{c}$.

j) Weight of 1,000 grains. $-24.7 \mathrm{~g}$.

k) Volume of 1,000 grains.-17.5c.c.

m) Thickness of bran layer. $(\mu)$

$\begin{array}{ccc}\text { averase. } & \text { max. } & \text { min. } \\ 43.21 & 51.08 & 39.83\end{array}$

p) Chemical composition :--

$\begin{array}{lr}\text { Water } & \mathbf{1 4 . 4 1} \\ \text { Stareh } & \mathbf{7 4 . 9 5} \\ \text { Sugar } & 1.35 \\ \text { Crude protein } & 7.75 \\ \text { "' fat } & 1.64 \\ \text { Ash } & 1.09\end{array}$

U) Production of Akita prefecture.

a) Percentage of full grown grains. - 95.1

b) External appearance and luster._- Same as $T$.

c) Size of grains. (m.m.)

\begin{tabular}{|c|c|c|c|c|c|}
\hline thick & above & 2.2 & $80.2 \mathrm{~g}$. & $40 \mathrm{~g}$ & rains. \\
\hline "I & 11 & 2.0 & $18.2 "$ & 9 & $\prime \prime$ \\
\hline 11 & $" 1$ & 1.8 & $1.5 " \prime$ & 1 & "I \\
\hline " & under & 1.8 & $0.1 " \prime$ & - & \\
\hline & & average. & $\max$. & $\min$ & \\
\hline & ngth & 5.15 & 5.75 & 4.80 & \\
\hline & dth & 3.17 & 3.40 & 300 & \\
\hline
\end{tabular}

d) Percentage of Shinziromai.-16.8

e) Depth of longitudinal furrow. $(\mu)$

$\begin{array}{rrr}\text { average. } & \max . & \min . \\ 5493 & 77.85 & 25.95\end{array}$

f) Specific gravity.-1.409

h) Hardness. (kilo)

$\begin{array}{ccc}\text { average. } & \max . & \min . \\ 5.20 & 8.16 & 3.15\end{array}$

i) Volume of $100 \mathrm{~g}$ - $125.5 \mathrm{c} . \mathrm{c}$.

j) Weight of 1,000 grains $-24.95 \mathrm{~g}$.

k) Volume of 1,000 grains. - $17.8 \mathrm{c} . \mathrm{c}$.

m) Thickness of bran layer. $(\mu)$

$\begin{array}{ccc}\text { average. } & \max . & \min . \\ 43.93 & 50.40 & 36.00\end{array}$

p) Chemical composition :-

Water

Starch 


$\begin{array}{cc}\text { Sugar } & 1.20 \\ \text { Crude protein } & 8.81 \\ \text { " fat } & 1.95 \\ \text { Ash } & 1.06\end{array}$

V) Production of Akita prefecture.

a) Percentage of full grown grains. -95.1

b) External appearance and luster.-Same as U).

c) Size of grains. (m.m.)

\begin{tabular}{|c|c|c|c|c|c|}
\hline thick & s above & 2.2 & $77.5 \mathrm{~g}$. & $39 \xi$ & grains. \\
\hline 11 & $\prime \prime$ & 2.0 & $17.2 "$ & 9 & $\prime \prime$ \\
\hline$\prime \prime$ & $\prime \prime$ & 1.8 & $4.3^{\prime \prime}$ & 2 & "' \\
\hline$\prime \prime$ & under & 1.8 & $0.9 \prime \prime$ & - & \\
\hline & & average. & max. & $\min$. & \\
\hline & ength & 5.13 & 5.60 & 4.85 & \\
\hline & ididh & 3.15 & 3.40 & 2.95 & \\
\hline
\end{tabular}

d) Percentage of Shinziromai._4.0

e) Depth of longitudinal furrow. ( $\mu$ )

$\begin{array}{ccc}\text { average. } & \max , & \min . \\ 48.87 & 77.85 & 17.30\end{array}$

f) Specific gravity.- 1.394

h) Hardness. (kilo)

$\begin{array}{ccc}\text { average. } & \max . & \min . \\ 4.73 & 6.81 & \end{array}$

i) Volume of $100 \mathrm{~g} .-126.5 \mathrm{c} . \mathrm{c}$.

j) Weight of 1,000 grains. $-24.6 \mathrm{~g}$.

k) Volume of 1,000 grains.-17.55c.c.

m) Thickness of bran layer. $(\mu)$

$\begin{array}{ccc}\text { average. } & \max . & \min . \\ 42.60 & 48.15 & 38.48\end{array}$

p) Chemical composition :-

$\begin{array}{lr}\text { Water } & 14.9 \pm \\ \text { Starch } & 75.48 \\ \text { Sugar } & 0.76 \\ \text { Crude protein } & 9.90 \\ \prime \prime \text { fat } & 1.01 \\ \text { Ash } & 0.84\end{array}$

W) Production of Yamagata prefecture.

a) Percentage of full grown grains. -92.3

b) External appearance and luster.- Same as V).

c) Size of grains. (m.m.)

thickness above

" $" 1$

" $" 1$

1/ under
2.2

2.0

1.8

1.8
37.3 g.

$45.2 \prime \prime$

$15.5 \%$

$1.9 "$
19 grains.

2311

$8 \quad 1$ 


$\begin{array}{lrrr} & \text { average. } & \max . & \min . \\ \text { length } & 5.04 & 5.35 & 4.60 \\ \text { width } & 3.01 & 3.30 & 2.70\end{array}$

d) Percentage of Shinziromai, -1.0

e) Depth of longitudinal furrow. $(\mu)$

$\begin{array}{rrr}\text { average. } & \max . & \min . \\ 57.74 & 86.50 & \mathbf{3 4 . 6 0}\end{array}$

f) Specific gravity.-1.418

h) Hardness. (kilo)

$\begin{array}{ccc}\text { average. } & \max . & \min . \\ 6.30 & 7.95 & 3.49\end{array}$

i) Volume of $100 \mathrm{~g}$. 127.0 c.c.

j) Weight of 1,000 grains._-22.0g.

k) Volume of 1,000 grains.-15.6c.c.

m) Thickness of bran layer. $(\mu)$

$\begin{array}{ccc}\text { average. } & \max . & \min . \\ 40.90 & 46.58 & 33.75\end{array}$

p) Chemical composition :-

$\begin{array}{lr}\text { Water } & 13.70 \\ \text { Starch } & 73.98 \\ \text { Sugar } & 1.80 \\ \text { Crude protein } & 8.82 \\ \quad \text { fat } & 1.91 \\ \text { Ash } & 1.12\end{array}$

ß) Polished lice, suitable for the Sake brewing. 23 (A-W) varieties.

A) Production of Okayama prefecture.

a) Percentage of full grown grains. -94.2

b) External appearance and luster.-White glassy luster, grains were fat and large. 1st class.

p) Chemical composition :-

$\begin{array}{lrlr}\text { Water } & 13.20 & \text { Starch } & 75.64 \\ \text { Sugar } & 0.46 & \text { Crude protein } & 9.04 \\ \text { Crude fat } & 0.37 & \text { Ash } & 0.35\end{array}$

B) Production of Niigata prefecture.

a) Percentage of full grown grains.- $\mathbf{9 7 . 0}$

b) External appearance and luster.- Nearly same as A).

p) Chemical composition :-

$\begin{array}{lrlr}\text { Water } & 15.63 & \text { Starch } & 74.38 \\ \text { Sugar } & 0.61 & \text { Crude protein } & 7.32 \\ \text { Crude fat } & 0.54 & \text { Ash } & 0.37\end{array}$

C) Production of Osaka prefecture. 
a) Percentage of full grown grains. -97.8

b) External appearance and luster.- Nearly same as A).

p) Chemical comosition :-

$\begin{array}{lrlr}\text { Water } & 13.20 & \text { Starch } & 78.16 \\ \text { Sugar } & 0.41 & \text { Crude protein } & 7.53 \\ \text { Crude fat } & 0.28 & \text { Ash } & 0.38\end{array}$

D) Production of Hyogo prefecture.

a) Percentage of full grown grains. $\quad 81.6$

b) External appearance and luster.-Same as C).

p) Chemical composition :-

$\begin{array}{lrlr}\text { Water } & 12.80 & \text { Starch } & 76.63 \\ \text { Sugar } & 0.61 & \text { Crude protein } & 7.10 \\ \text { Crude fat } & 0.58 & \text { Ash } & 0.35\end{array}$

E) Production of Hyogo prefecture.

a) Percentage of full grown grains.-90.4

b) External appearance and luster. Same as A).

p) Chemical composition :-

$\begin{array}{lrlr}\text { Water } & 12.00 & \text { Starch } & 77.23 \\ \text { Sugar } & 0.81 & \text { Crude protein } & 8.39 \\ \text { Crude fat } & 0.17 & \text { Ash } & 0.33\end{array}$

F) Production of Hyoyo prefecture.

a) Percentage of full grown grains. -96.6

b) External appearance and luster._- Same as A).

p) Chemical composition :-

$\begin{array}{lrlr}\text { Water } & 13.60 & \text { Starch } & 77.89 \\ \text { Sugar } & 0.46 & \text { Crude portein } & 7.32 \\ \text { Crude fat } & 0.52 & \text { Ash } & 0.24\end{array}$

G) Production of Oḱayama prefecture.

a) Percentage of full grown grains -88.8

b) External appearance and luster.—Same as A).

p) Chemical composition :-

$\begin{array}{lrlr}\text { Water } & 13.90 & \text { Starch } & 75.71 \\ \text { Sugar } & 0.38 & \text { Crude protein } & 8.18 \\ \text { Crude fat } & 0.38 & \text { Ash } & 0.31\end{array}$

H) Production of Okayama prefecture.

a) Percentage of full grown grains. -77.4

b) External appearance and Iuster.-Nearly same as G).

p) Chemical composition :- 


$\begin{array}{lrlr}\text { Water } & 12.60 & \text { Starch } & 74.51 \\ \text { Sugar } & 0.46 & \text { Crude protein } & 7.10 \\ \text { Crude fat } & 0.44 & \text { Ash } & 0.41\end{array}$

I) Production of Okayama prefecture.

a) Percentage of full grown grains. -96.4

b) External appearance and luster.—Same as A).

p) Chemical composition :-

$\begin{array}{lrlr}\text { Water } & 12.50 & \text { Starch } & 75.74 \\ \text { Sugar } & 0.33 & \text { Crude protein } & 7.96 \\ \text { Crude fat } & 0.35 & \text { Ash } & 0.34\end{array}$

J) Production of Hirostima prefecture.

a) Percentage of full grown grains.- 97.4

b) External appearance and luster.-Same as A).

p) Chemical composition :-

$\begin{array}{lrlr}\text { Water } & 12.30 & \text { Starch } & 75.46 \\ \text { Sugar } & 0.66 & \text { Crude protein } & 8.54 \\ \text { Crude fat } & 0.65 & \text { Ash } & 0.39\end{array}$

K) Production of Okayama prefecture.

a) Percentage of full grown grains.-95.4

b) External appearance and luster.- Nearly same as J).

p) Chemical composition :-

$\begin{array}{lrlr}\text { Water } & 12.40 & \text { Starch } & 76.00 \\ \text { Sugar } & 0.31 & \text { Crude protein } & 6.89 \\ \text { Crude fat } & 0.18 & \text { Ash } & 0.89\end{array}$

L) Production of Okayama prefecture.

a) Percentage of full grown grains.- 96.4

b) External appearance and luster.-Same as A).

p) Chemical composition :-

$\begin{array}{lrlr}\text { Water } & 12.00 & \text { Starch } & 74.65 \\ \text { Sugar } & 0.31 & \text { Crude protein } & 6.67 \\ \text { Crude fat } & 0.32 & \text { Ash } & 0.32\end{array}$

M) Production of Okayama prefecture.

a) Percentage of full grown grains.-- 87.6

b) External appearance and luster.-- - Nearly same as L).

p) Chemical composition :-

$\begin{array}{lrlr}\text { Water } & 14.70 & \text { Starch } & 77.93 \\ \text { Sugar } & 0.41 & \text { Crude protein } & 7.75 \\ \text { Crude fat } & 0.14 & \text { Ash } & 0.31\end{array}$


N) Production of Okayama prefecture.

a) Percentage of full grown grains.- 86.2

b) External appearance and luster.— Nearly same as A).

p) Chemical composition :-

$\begin{array}{lrlr}\text { Water } & 14.20 & \text { Stareh } & 76.76 \\ \text { Sugar } & 0.46 & \text { Crude protein } & 7.96 \\ \text { Crude fat } & 0.50 & \text { Ash } & 0.38\end{array}$

O) Production of Kumamoto prefecture.

a) Percentage of full grown grains.- -84.4

b) External appearance and luster.-Nearly same as A).

p) Chemical composition :-

$\begin{array}{lrlr}\text { Water } & 13.40 & \text { Starch } & 76.94 \\ \text { Sugar } & 0.26 & \text { Crude protein } & 8.54 \\ \text { Crude fat } & 0.23 & \text { Ash } & 0.95\end{array}$

P) Production of Kumamoto prefecture.

a) Percentage of full grown grains. - 90.8

b) External appearance and luster.-Same as A).

p) Chemical composition :-

$\begin{array}{lrlr}\text { Water } & 12.60 & \text { Starch } & 78.18 \\ \text { Sugar } & 0.38 & \text { Crude protein } & 6.45 \\ \text { Crude fat } & 0.26 & \text { Ash } & 0.33\end{array}$

Q) Production of Kumamoto prefecture.

a) Percentage of full grown grains. - 88.2

b) External appearance and luster.- - Same as P).

p) Chemical composition :-

$\begin{array}{lrlr}\text { Water } & 12.40 & \text { Starch } & 76.88 \\ \text { Sugar } & 0.33 & \text { Crude protein } & 8.54 \\ \text { Crude fat } & 0.31 & \text { Ash } & 0.31\end{array}$

R) Production of Kumamoto prefecture.

a) Percentage of full grown grains.- 89.6

b) External appearance and luster.—Same as Q).

p) Chemical composition :-

$\begin{array}{lrlr}\text { Water } & 12.30 & \text { Starch } & 75.79 \\ \text { Sugar } & -0.28 & \text { Crude protein } & 7.32 \\ \text { Crude fat } & 0.15 & \text { Ash } & 0.38\end{array}$


S) Production of Akita prefecture.

a) Percentage of full grown grains.-94.6

b) External appearance and luster.-Nearly same as A).

p) Chemical composition :-

$\begin{array}{lrlr}\text { Water } & 12.40 & \text { Starch } & 74.92 \\ \text { Sugar } & 0.76 & \text { Crude protein } & 8.18 \\ \text { Crude fat } & 0.11 & \text { Ash } & 0.31\end{array}$

T) Production of Akita prefecture.

a) Percentage of full grown grains. -97.1

b) External appearance and luster.-Same as S).

p) Chemical composition :-

$\begin{array}{lrlr}\text { Water } & 13.90 & \text { Starch } & 75.68 \\ \text { Sugar } & 0.41 & \text { Crude protein } & 7.75 \\ \text { Crude fat } & 0.29 & \text { Ash } & 0.29\end{array}$

U) Production of Akita prefecture.

a) Percentage of full grains._- 99.4

b) External appearance and luster.-Same as T).

p) Chemical composition :-

$\begin{array}{lrlr}\text { Water } & 13.10 & \text { Starch } & 77.55 \\ \text { Sugar } & 0.46 & \text { Crude protein } & 7.32 \\ \text { Crude fat } & 0.33 & \text { Ash } & 0.31\end{array}$

V) Production of Akita prefecture.

a) Percentage of full grown grains. -94.8

b) External appearance and luster.- Same as U).

p) Chemical composition :-

$\begin{array}{lrlr}\text { Water } & 14.40 & \text { Starch } & 75.64 \\ \text { Sugar } & 0.46 & \text { Crude protein } & 7.32 \\ \text { Crude fat } & 0.36 & \text { Ash } & 0.26\end{array}$

W) Production of Yamagata prefecture.

a) Percentage of full grown grains. -98.0

b) External appearance and luster. Nearly same as A).

p) Chemical composition :-

$\begin{array}{lrlr}\text { Watex } & 14.60 & \text { Starch } & 75.79 \\ \text { Sugar } & 0.41 & \text { Crude protein } & 7.75 \\ \text { Crude fat } & 0.50 & \text { Ash } & 0.46\end{array}$




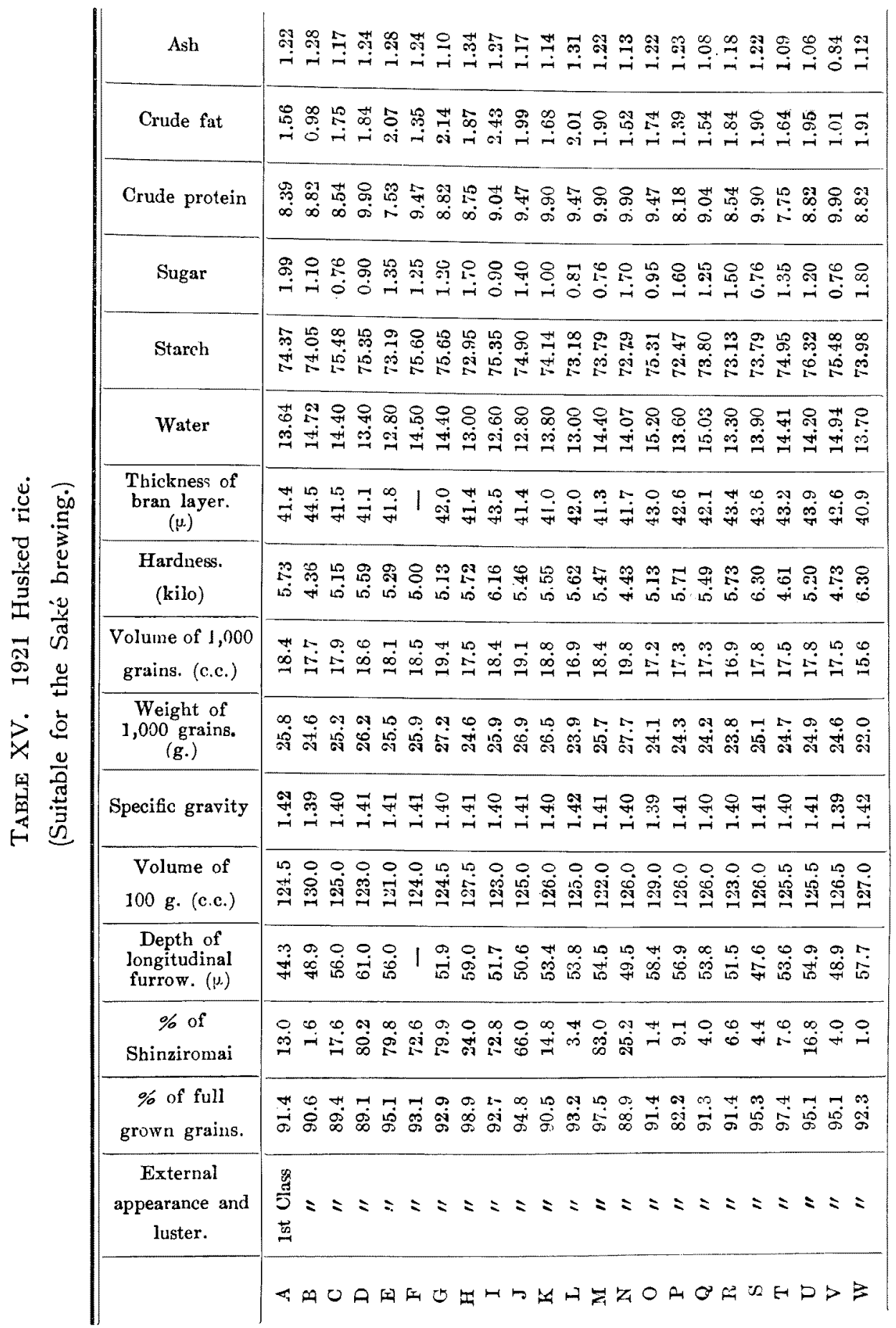


TABLE XVI. 1921 Polished rice.

(Suitable for the Sake brewing.)

\begin{tabular}{c|c|c|c|c|c|c|c|c}
\hline & $\begin{array}{r}\text { External } \\
\text { appearance } \\
\text { and luster of full } \\
\text { grown } \\
\text { grains. }\end{array}$ & Water & Starch & Sugar & $\begin{array}{c}\text { Crude } \\
\text { protein }\end{array}$ & $\begin{array}{c}\text { Crude } \\
\text { fat }\end{array}$ & Ash \\
\hline A & Ist Class & 94.2 & 13.20 & 75.64 & 0.46 & 9.04 & 0.37 & 0.35 \\
B & $\prime \prime$ & 97.0 & 15.63 & 74.38 & 0.61 & 7.32 & .0 .54 & 0.37 \\
C & $\prime \prime$ & 97.8 & 13.20 & 78.16 & 0.41 & 7.53 & 0.28 & 0.38 \\
D & $\prime \prime$ & 81.6 & 12.80 & 76.63 & 0.61 & 7.10 & 0.58 & 0.35 \\
E & $\prime \prime$ & 90.4 & 12.00 & 77.23 & 0.81 & 8.39 & 0.17 & 0.33 \\
F & $\prime \prime$ & 96.6 & 13.60 & 77.89 & 0.46 & 7.32 & 0.52 & 0.24 \\
G & $\prime \prime$ & 88.8 & 13.90 & 75.71 & 0.38 & 8.18 & 0.38 & 0.31 \\
H & $\prime \prime$ & 77.4 & 12.60 & 74.51 & 0.46 & 7.10 & 0.44 & 0.41 \\
I & $\prime \prime$ & 96.4 & 12.50 & 75.74 & 0.33 & 7.96 & 0.35 & 0.31 \\
J & $\prime \prime$ & 97.4 & 12.30 & 75.46 & 0.66 & 8.54 & 0.65 & 0.39 \\
K & $\prime \prime$ & 95.4 & 12.40 & 76.00 & 0.31 & 6.89 & 0.18 & 0.89 \\
L & $\prime \prime$ & 96.4 & 12.00 & 74.65 & 0.31 & 6.67 & 0.32 & 0.32 \\
II & $\prime \prime$ & 87.6 & 14.70 & 77.93 & 0.41 & 7.75 & 0.14 & 0.31 \\
N & $\prime \prime$ & 86.2 & 14.20 & 76.76 & 0.46 & 7.96 & 0.50 & 0.38 \\
O & $\prime \prime$ & 84.4 & 13.40 & 76.94 & 0.26 & 8.54 & 0.23 & 0.95 \\
P & $\prime \prime$ & 90.8 & 12.60 & 78.18 & 0.38 & 6.45 & 0.26 & 0.33 \\
Q & $\prime \prime$ & 88.2 & 12.40 & 76.88 & 0.33 & 8.54 & 0.31 & 0.31 \\
R & $\prime \prime$ & 89.6 & 12.30 & 75.79 & 0.28 & 7.32 & 0.15 & 0.38 \\
S & $\prime \prime$ & 94.6 & 12.40 & 74.92 & 0.76 & 8.18 & 0.11 & 0.31 \\
T & $\prime \prime$ & 97.1 & 13.90 & 75.68 & 0.41 & 7.75 & 0.29 & 0.29 \\
U & $\prime \prime$ & 99.4 & 13.10 & 77.55 & 0.46 & 7.32 & 0.33 & 0.31 \\
V & $\prime \prime$ & 94.8 & 14.40 & 75.64 & 0.46 & 7.32 & 0.36 & 0.26 \\
W & $\prime \prime$ & 98.0 & 14.60 & 75.79 & 0.41 & 7.75 & 0.50 & 0.46 \\
\hline & & & & & & & & \\
\hline
\end{tabular}

\section{Summary of the 1921 production.}

The suitable materials showed fineluster and external appearance, and in the size of grains, percentage of both full grown grains and Shinziromai, both weight and volume of 1,000 grains, hardness and the contents of carbohydrates, and crude protein showed nearly equal or somewhat higher value compared with the suitable materials of the production of the preceding years. On the other hand, in the depth of longitudinal furrow, volume of $100 \mathrm{~g}$., thickness of bran layer, and the contents of crude fat and ash showed the production of this year a smaller value than those of the preceding years, while in the specific gravity and water a moderate number was observed. 
Nos. 9-12.]

\begin{tabular}{|c|c|c|c|c|c|c|c|c|}
\hline & 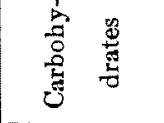 & $\begin{array}{l}\infty \\
\text { s. } \\
\text { s. } \\
15\end{array}$ & $\begin{array}{l}8 \\
87 \\
0 \\
03\end{array}$ & 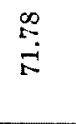 & $\begin{array}{l}19 \\
9 \\
10 \\
10\end{array}$ & $\begin{array}{l}\overrightarrow{6} \\
\dot{8}\end{array}$ & 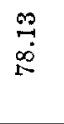 & $\begin{array}{l}g \\
\stackrel{0}{0} \\
0\end{array}$ \\
\hline & 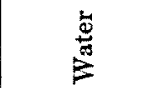 & 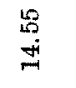 & $\begin{array}{l}\stackrel{g}{\circ} \\
\stackrel{+}{-1}\end{array}$ & $\begin{array}{l}89 \\
\substack{19 \\
-1}\end{array}$ & 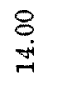 & $\begin{array}{l}5 \\
6 \\
05 \\
20\end{array}$ & $\begin{array}{l}\stackrel{g}{g} \\
\stackrel{+}{-}\end{array}$ & $\begin{array}{l}\stackrel{8}{8} \\
\text { m }\end{array}$ \\
\hline $\begin{array}{l}\dot{\hat{A}} \\
\dot{s}\end{array}$ & 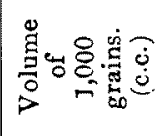 & $\begin{array}{l}0 \\
\infty \\
\infty\end{array}$ & $\vec{\Leftrightarrow}$ & $\begin{array}{l}\infty \\
0 \\
= \\
=1\end{array}$ & $\stackrel{\circ}{\stackrel{\circ}{0}}$ & $\stackrel{\circ}{\underset{-}{2}}$ & $\stackrel{g}{\stackrel{+}{+}}$ & $\begin{array}{l}10 \\
0 \\
0\end{array}$ \\
\hline 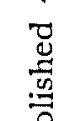 & 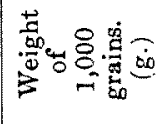 & İ & à & 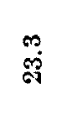 & 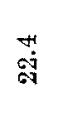 & ڤุ่ & $\frac{9}{9}$ & 옹 \\
\hline $\overrightarrow{\dot{U}}$ & 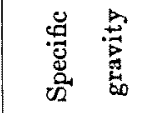 & 我 & 葛 & $\begin{array}{l}5 \\
\infty \\
\infty \\
\\
-1\end{array}$ & 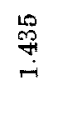 & 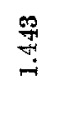 & $\underset{\mathbb{H}}{\stackrel{9}{+}}$ & 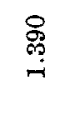 \\
\hline 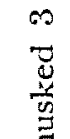 & 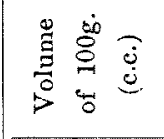 & 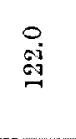 & 总 & 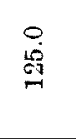 & \begin{tabular}{l}
$\stackrel{0}{0}$ \\
\hdashline
\end{tabular} & $\stackrel{\stackrel{0}{0}}{\because}$ & $\begin{array}{l}\stackrel{\circ}{0} \\
\stackrel{\infty}{\Rightarrow}\end{array}$ & $\stackrel{\circ}{\stackrel{+}{J}}$ \\
\hline . & 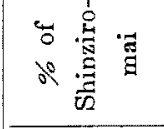 & 节 & $\stackrel{ }{\circ}$ & ị & $\frac{0}{9}$ & $\stackrel{\leftrightarrow}{\leftrightarrow}$ & 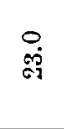 & $\begin{array}{l}8 \\
0 \\
0\end{array}$ \\
\hline 焉 & 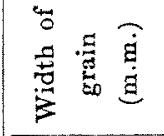 & 苛 & $\begin{array}{l}89 \\
8 j \\
6 j\end{array}$ & $\begin{array}{l}8 \\
\infty \\
\infty\end{array}$ & $\begin{array}{l}\vec{\sigma} \\
\sigma i\end{array}$ & $\begin{array}{l}8 \\
8 \\
a\end{array}$ & $\begin{array}{l}\infty \\
\infty \\
\infty \\
o j\end{array}$ & $\begin{array}{l}\mathscr{O} \\
\sigma \\
-i\end{array}$ \\
\hline 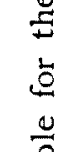 & 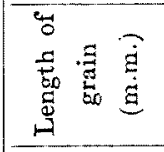 & $\underset{0}{8}$ & $\frac{9}{40}$ & $\begin{array}{l}8 \\
\text { is }\end{array}$ & $\underset{4}{\stackrel{H}{4}}$ & 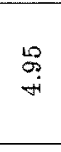 & 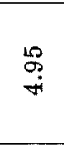 & $\stackrel{9}{10}$ \\
\hline 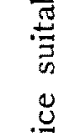 & 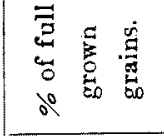 & $\begin{array}{l}\infty \\
\ddot{\infty} \\
\infty\end{array}$ & $\begin{array}{l}i \\
\text { si } \\
o\end{array}$ & $\stackrel{\circ}{\dot{x}}$ & $\begin{array}{c}\infty \\
\substack{\infty \\
\infty}\end{array}$ & $\begin{array}{ll}0 \\
\infty \\
\infty \\
\infty\end{array}$ & $\underset{\infty}{H}$ & $\begin{array}{l}\infty \\
\text { sj } \\
\delta\end{array}$ \\
\hline 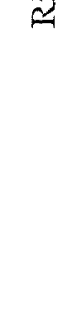 & & 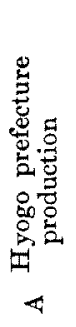 & 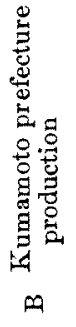 & 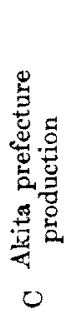 & 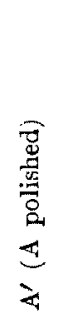 & 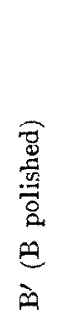 & 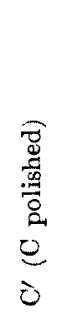 & 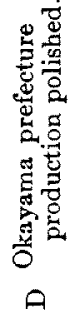 \\
\hline
\end{tabular}


[Vol. 3.

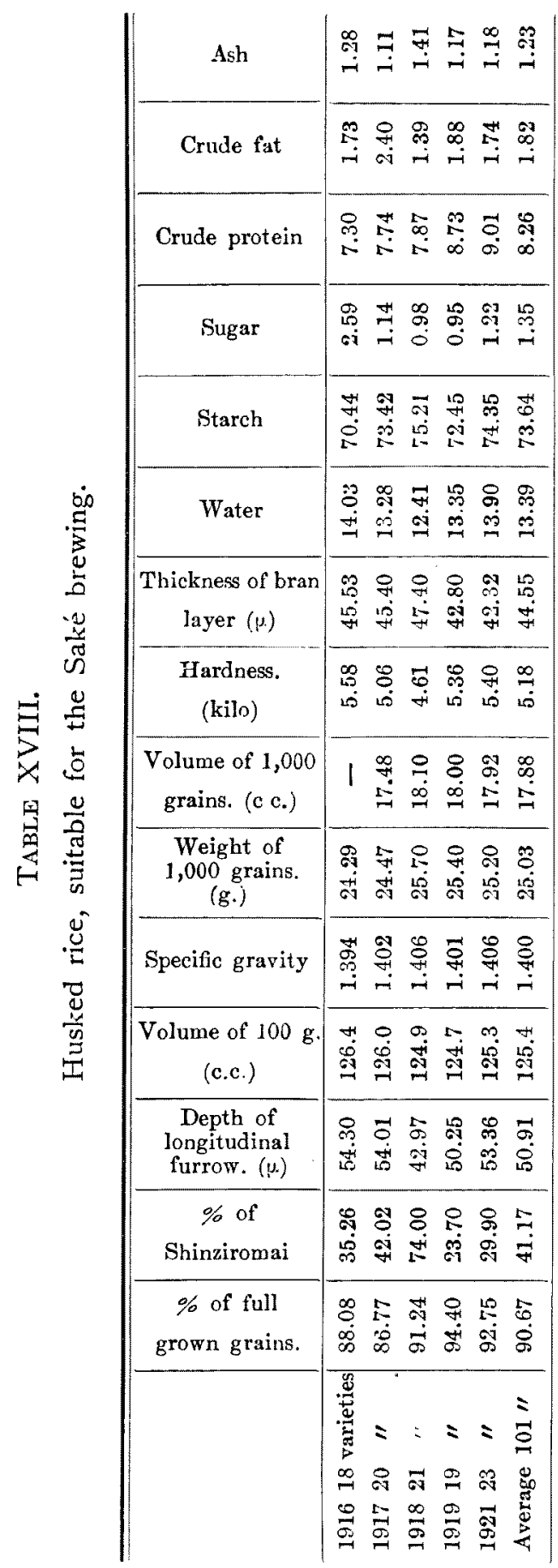

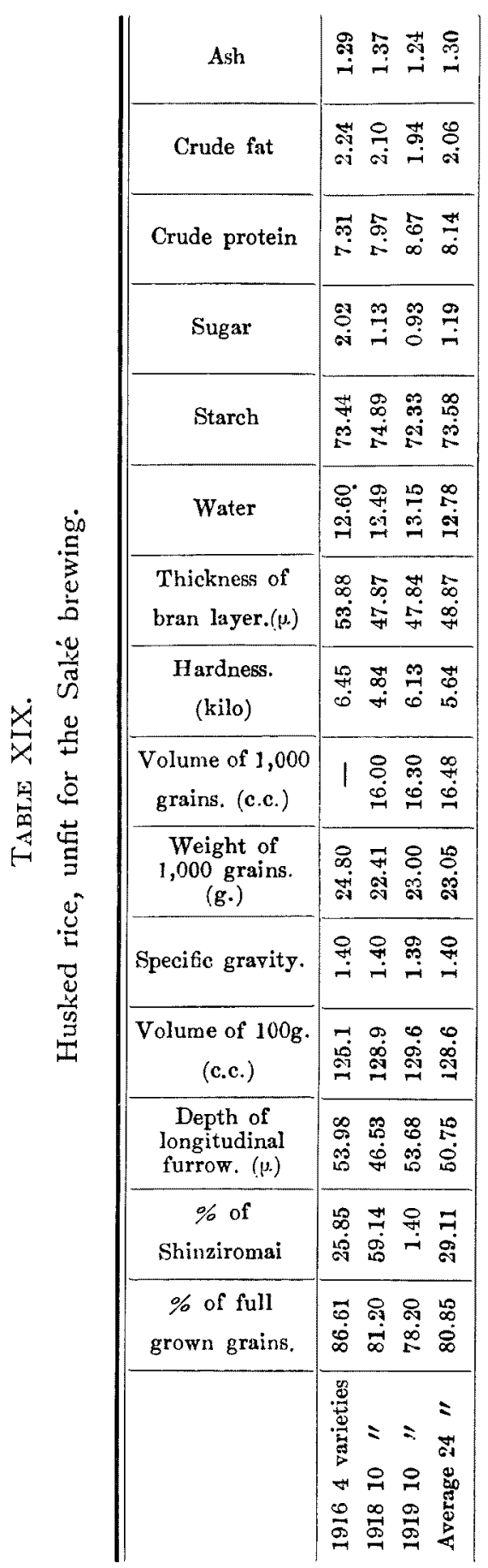




\begin{tabular}{|c|c|c|}
\hline & Ash & 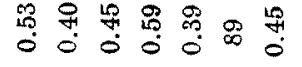 \\
\hline & Crude fat & 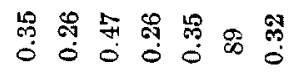 \\
\hline & Crude protein & 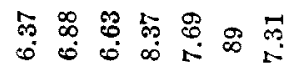 \\
\hline & Sugar & 묭 \\
\hline & Starch & 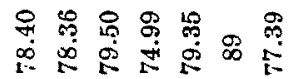 \\
\hline . & Water & 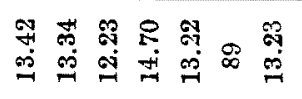 \\
\hline 宸 & Hardness. (kilo) & 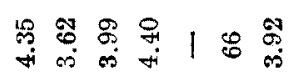 \\
\hline 肴 & $\begin{array}{c}\text { Volume of } 1,000 \\
\text { grains. (c.c.) }\end{array}$ & 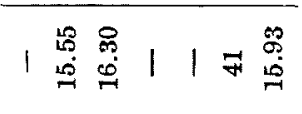 \\
\hline 乐 & $\begin{array}{l}\text { Weight of } \\
1,000 \text { grains. } \\
\text { (g.) }\end{array}$ & 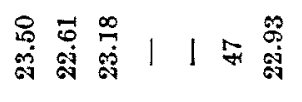 \\
\hline 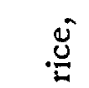 & Specific gravity & 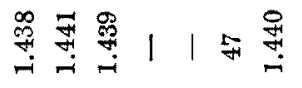 \\
\hline$\frac{d}{0}$ & $\begin{array}{l}\text { Volume of } 100 \mathrm{~g} . \\
\text { (c.c.) }\end{array}$ & 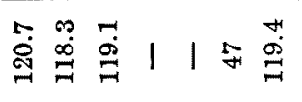 \\
\hline & $\begin{array}{l}\text { Depth of } \\
\text { longitudinal } \\
\text { furrow. }(\mu .)\end{array}$ & 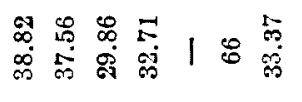 \\
\hline & $\begin{array}{c}\% \text { of } \\
\text { Shinziromai }\end{array}$ & 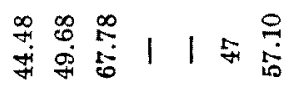 \\
\hline & $\begin{array}{l}\% \text { of full } \\
\text { grown grains. }\end{array}$ & 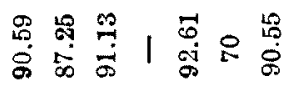 \\
\hline & & 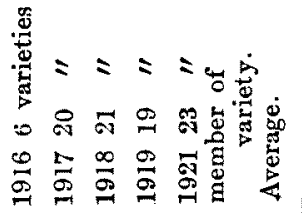 \\
\hline
\end{tabular}

\begin{tabular}{|c|c|c|}
\hline & Asb & 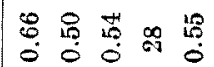 \\
\hline & Crude fat & 密 里 \\
\hline & Crude protein & 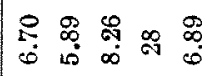 \\
\hline & Sugar & 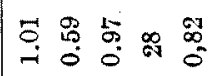 \\
\hline & Starch & 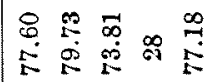 \\
\hline ڤై & Water & 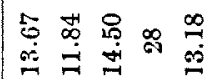 \\
\hline 苋 & Hardness. (kilo) & 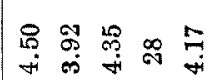 \\
\hline $\begin{array}{l}\dot{ \pm} \\
\dot{x} \\
\end{array}$ & $\begin{array}{c}\text { Volume of } 1,000 \\
\text { grains. (c.c.) }\end{array}$ & 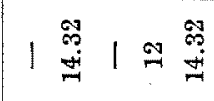 \\
\hline 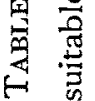 & $\begin{array}{l}\text { Weight of } \\
1,000 \text { grains. } \\
\text { (g.) }\end{array}$ & $\begin{array}{l}\infty \\
\infty \\
\stackrel{9}{*} \\
\stackrel{9}{0}\end{array}$ \\
\hline 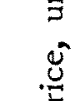 & Specific gravity. & 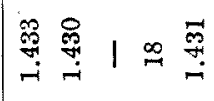 \\
\hline$\frac{0}{\mathscr{O}}$ & $\begin{array}{c}\text { Volume of } 100 \mathrm{~g} \text {. } \\
\text { (c.c.) }\end{array}$ & 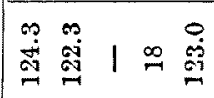 \\
\hline & $\begin{array}{l}\text { Depth of } \\
\text { longitudinal } \\
\text { furrow. }(\mu .)\end{array}$ & 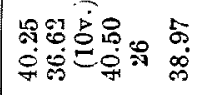 \\
\hline & $\begin{array}{l}\% \text { of } \\
\text { Shinziromai. }\end{array}$ & 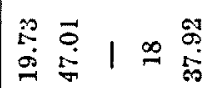 \\
\hline & $\begin{array}{l}\% \text { of full } \\
\text { grown grains. }\end{array}$ & 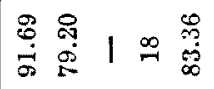 \\
\hline & & 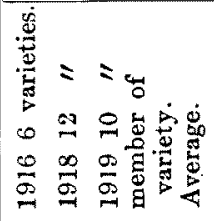 \\
\hline
\end{tabular}




\section{Conchusions.}

As the results of investigations on 242 varieties (husked 125, polished 117) of rice used in Sake brewing, the following conclusions are drawn.

1. Both luster and external appearance ought to be fine for the brewing rice just as for the food stuff. The colour should be light and the shape, large and fat. The impurities must be as little as possible.

2. Full grown grains :- The suitable materials contain over $90 \%$ of full grown grains, while the unsuitable about $80 \%$.

3. Size of grains :- Conveniently calculated from the volume and weight of 1,000 grains. The volume and weight of 1,000 grains are $17.88 \mathrm{c}$.c. and $25.03 \mathrm{~g}$, respectively in the suitable materials, whilst $16.48 \mathrm{c} . \mathrm{c}$. and $23.05 \mathrm{~g}$. in the unsuitable.

4. Percentage of Shinziromai :- This varies according to the year of the production, but in the materials of the production of the same year, the suitable materials are, in many case, rich in Shinziromai, namely, the suitable materials contain $41.17 \%$ of it while the unsuitable $29.11 \%$.

5. Volume of $100 \mathrm{~g}$ :- The suitable materials occupy always a smaller volume $(125.4 \mathrm{c} . \mathrm{c}$.$) than the unsuitable (128.6 \mathrm{c} . \mathrm{c}$.$) .$

6. Specific gravity :- The suitable materials show somewhat greater value in the specific gravity than the unsuitable, except those of 1916 production. But the difference is so small that no reliable conclusion can be drawn on it, viz. the specific gravity of the suitable materials is 1.400 that of the unsuitable 1.3950 .

7. Hardness :- In hardness, suitable materials always give a lower value than the unsuitable. But an extermely soft matreial is not fitted for the brewing. It ought to be moderate. The suitable materials resist the pressure up to 5.175 kilograms while the unsuitable up to 5.649 kilograms.

8. Depth of longitudinal furrow :- This shows no material difference both in the suitable and unsuitable materials. The average depth of it is $50.91 \mu$ in the suitable materials and 50.75 in the unsuitable. Compared with their size, however, the suitable materials give a smaller value than the unsuitable.

9. Thickness of bran layer:- Suitable materials have always a thinner bran layer $(44.55 \mu)$ than the unsuitable ones $(48.86 \mu)$.

10. Water-Smaller contents of water would be desirable from chemical stand point, but, since too small contents of water make the grains too hard, so the moderate humidity is rather desirable. The suitable materials contain $13.39 \%$ of water and the unsuitable $12.78 \%$.

11. Carbohydrats (Starch, Dextrin, sugar) :- Suitable materials always indicate somewhat higher value $(83.178 \%)$ in the contents of carbohydrates 
than the unsuitable $(82.944 \%)$.

12. Crude protein :- Suitable materials indicate somewhat ligher contents of crude protein compared with the unsuitable materials. This is so not only in husked rice but in polished rice, too. But the contents fluctuate according to the year of the produce. The contents ought to be as little as possible, since the protein is a source of fusel oil. It may be profitable to obtain some definite standard for the protein contents and the mean value is 8.263 $\%$ for the suitable materials and $8.144 \%$ for the unsuitable.

13. Crude fats :- The suitable materials ought to contain the crude fats as little as possible. The mean value for the suitable materials is $1.822 \%$ and for the unsuitable $2.058 \%$.

14. Ash :- This too ought to be little. The mean value for the suitable materials is $1.23 \%$ and for the unsuitable $1.30 \%$.

15. Saccharifying quality and absorption of water ought to be moderate, while the contents of crude fibre are the better, the smaller. Saccharifying quality-Suitable material $32.56 \%$ and the unsuitable $39.08 \%$.

Absorption of water:- The suitable materials $22.33 \%$, and the unsuitable $22.88 \%$.

Crude fibre.-The suitable materials $1.2786 \%$, and the unsuitable $1.292 \%$.

Phosphoric acid:- The suitable materials $0.4989 \%$ and the unsuitable $0.4735 \%$.

Phytin :- Phosphorus estimated as $\mathrm{P}_{2} \mathrm{O}_{5}$;

The husked rice (suitable) $0.4323 \%$.

The polished rice (suitable) $0.0374 \%$.

\section{Reference (polished rice).}

1. Both luster and external appearance :- Just in the same nanner as in the husked rice.

2. Full grown grains:- The suitable materials show above $90 \%$, while the unsuitable about $83 \%$.

3. Size of grains:- In the same manner as in the husked rice-The suitable materials $22.93 \mathrm{~g}$. 15.93c.c. the unsuitable $20.57 \mathrm{~g}$. 14.32c.c.

4. Percentage of shinziromai :- The suitable materials 57.10, while the unsuitable 37.92 .

5. Volume of $100 \mathrm{~g}$ The suitable materials indicate 119.4 c.c. while the unsuitable $123.0 \mathrm{c} . \mathrm{c}$.

6. Specific gravity-The suitable materials 1.44 , the unsuitable 1.431 .

7. Hardess :- The suitable materials 3.915 kilo, while the unsuitable 4.170 kilo.

8. Depth of longitudinal furrow:- The suitable materials $33.37 \mu$, the 
unsuitable $38.97 \mu$.

9. Water :- The suitable materials $13.23 \%$, the unsuitable $13.18 \%$.

10, Carbohydrates(Starch, Dextrin, Sugar)-The suitable materials 86.6662 $\%$, the unsuitable $86.5707 \%$.

11. Crude protein:- The suitable materials $7.3148 \%$, the unsuitable $6.8874 \%$

12. Crude fat :- The suitable materials $0.3152 \%$, the unsuitable $0.4888 \%$.

13. Ash :- The suitable materials $0.4508 \%$, the unsuitable $0.5457 \%$

\section{LITERAtURE.}

1. O. Kellner, Tanaka, Kobayashi:- Report of Tokio Imperial Collage of Agriculture and Forestry. No. 5.

Distribution of nutritious matters among rice by polishing process. They determined general chemical components of polished and crushed rice and bran.

2 O. Kallner, M. Nagaoka:- Bulletin of the collage of Agricultnre No. 12. Analysis of rice grain. Nine varieties of husked rice were analysed physically and chemically, also constituents of ash were determined. They concluded that market price of rice has no relation to its chemical composition, but the rice of good quality is rich in nitrogen contents.

3. M. Sawamura:- Journal of the Scientific Agricultural Society. No. 51.

Relation between quality and chemical constituents of rice. He obtained nearly the same result with the former. He added that rice of inferior quality is rich in fat and ash and polished rice of inferior quality gave much water extract.

4. L. Grandeaŭ, C. de Leeuw, Wagner, O. Kellner, König, W. Pillitz, Brimmer, Hanamann, A. Petermann, F. Strohmer, N. V. Lorenz, Soxhlet, Henkel, Flourens, Berger, Meissl, Balland, Wattson, E. H. Jenkins, Richardson, U. Kreusler, J. B. Boussingault, A. R. Ledaux, Becke, Cosack, W. Kisch, Stift, Branberg, Uchiyama, Yamada, Nagaoka, Tsujioka, Saito, Sasaki, Sawano, Yoshii, Makino:- König's Nahrungs und Genussmittel 1903. They conducted chemical analysis of rice for about ten years since 1871 .

5. Report of Osaka Sanitary Experiment Station 1891. Korean, Siam and Annam rice analysed chemically.

6. Rosenheim, Kajiura :- J. of Physiol. 36 liv-lv. 1908. They investigated protein matters in rice and obtained albumin, globulin and glutenin, but could not find prolamin, soluble in dilute ethyl alcohol. They named glutenin as oryzenin. The investigation was preliminary one, but further report has not yet been made. 
7. U. Suzuki, K. Yoshimura:- J. of the Collage of Agriculture Vol. I No. 1. On protein matters in rice. Almost the same time with the preceding article, they investigated protein matters in polished rice and bran, and obtained albumin, globulin, glutenin and prolamin. Further they isolated mono and diamino acids from glutenin by hydrolysis.

8. U. Suzuki, S. Matsunaga:- J. of the Collage of Agriculture Vol. V. No. 1.

On the presence of nicotinic acid in rice bran. They proved the presence of nicotinic acid in rice bran, which was once misunderstood by Funk as vitamin.

9. I. Inagaki, U. Suzuki, K. Aso:- J. of the Scient. Agricuí. Society. No. 47.

On "Harajiro" part of rice. "Harajiro" part contains less crude protein compared with the other part of rice. The ratio between them was 76.1 to $10^{\prime}$, while other constituents did not show any special difference.

10. K. Goto:- J. of Scient. Agricul. Society. No. 61.

Investigation on the quality of rice. Unripen rice when compared to full grown one indicates the less hardness, smaller specific gravity, and the smaller contents of crucle protein, crude fat and nitrogen free extract, while crude fibre and ash show greater value.

11. I. Inagaki :- J. of Scient. Agricul. Society. No. 91.

On the relation between market price and quality of rice. Qualities proportional to the market price are weight of full grown grains, weight of a certain volume, specific gravity aud hardness, while weight of unripe grains, bran, impurities other than rice grains and white part of rice are inversely proportional.

12. U. Suzuki :- J. of Scient. Agricult. Society. No. 94.

On distributions and conbined state of iron in the polished rice and bran. The polished rice contains $0.005 \%$ of iron while bran contains $0.1-0.13 \%$. Iron does not exist as an inorganic state but in an organic one. Bran contains much quantity of phosphorus, potash, magnesium and calcium compared with the polished rice.

13. U. Suzuki, T. Shimamura :- J. of Scient. Agricult. Society. No. 102, No. 103.

About an available composition aberi acid in rice bran. They found, in alcoholic extract of rice bran, an unknown indispensable nutritious constituent for growth of animals, and named it tentatively aberi acid.

14. B. Suzuki, T. Tanaka - J. of Scient. Agricult. Society. No. 129. 
Relation between the quality of rice and the constituents of bran. The bran of good rice is rich in nitrogen and fat, while poor in ash.

15. B. Suzuki, T. Tanaka :- J. of Scient. Agricult. Society. No.' 129. Constituents of rice bran. As the bran of good rice, surely, contains much quantity of fat, rice may be graded by the quantity of fat in the bran.

16. I. Inagaki :- J. of Scient. Agricult. Society. No. 137, No. 138.

On an appraisement of rice. The rice of good quality ought to be hard, thin husked and fat grain, and to have sharrow longitudinal furrow, high luster, the less moisture, less unripen grains etc.

17. M. Kondo:- J. of Scient. Agricult. Society. No. 153.

On a volume weight of husked rice.

(1) A volume weight of husked rice will increase when the surface of grain is smooth, when the shape of grain is short elliptic and round, when mixed with fine sand and the shape of grain is not uniform. (2) A volume weight of husked rice will decrease when the surface of grain is not smooth, when the shape of grain slender and flat, when mixed with broken and unripen grains. (3) The size and dryness of grains are indifferent to the volume weight.

18. M. Kondo, F. Oshiumi :- J. of Scient. Agricult. Society. No. 168.

- The relation between the volume weight and the degree of dryness of unhulled rice and husked rice.

(1) The volume weight is generally incraeased gradually according to dryness when the unhulled rice is dried by the the sun light or in a desiccator, while it is decreased when suddenly dried with artificial heat. (2) The volume weight is regularly increased according to the absorbed quantity of water by unhulled rice, quite independent of the way of drying. (3) The volume weight is decreased in proportional to the water content of husked rice which previously dried and kept to absorbe water in a moist atmosphere. This phenomenon is due chiefly to the swelling of grain than the increase of the weight of grain by the absorption of water.

19. I. Onodera :- J. of Scient. Agricult. Society. No. 180.

On the relation between the quality of rice and the fat contents of bran.

(1) The fat contents in the bran of better rice, are greater when compared to that of inferior ones, when difference of quality is grcat. (2) The fat contents of a full grown rice are greater than that of imperfectly matured ones. (3) When the larger grains are compared with the comparatively smaller grains, which constitute the greater part of 
the sample, the latter contain much quantity of fat.

20. M. Kondo:- J. of Scient. Agricult. Society. No. 185.

On the thickness of the bran layer of rice during the stage of ripening. (1) The thickness of the layer of bran differs according to the stage of ripening of rice. The thickness is the greatest in the milky ripening, then in yellow ripening becomes somewhat thinner, and afterwards no special change is observed. (2) The external layer (pericarp, testa) ${ }^{\circ}$ of bran is most thick in milky ripenig, afterwards it becomes thinner according to the advance of ripening. (3) The inner layer (perisperm, aleuron layer) of bran is most thin in milky ripening, afterwards it becomes thicker in accodance with the progress of ripening. (4) The bran layer is thicker in an inferior rice and thinner in a superior one.

21. S. Suzuki :- J. of Scient. Agricult. Society. No. 187.

The relation between the degree of polishing and the chemical composition of rice or barley. (1) Both protein and crude protein decrease their quantities regularly in accordance with the degree of polishing. (2) Crude fat, crude fibre, ash, phosphoric acid. lime and aryzanin decrease in a considerable degree during the first stage of polishing, but their degree of decreasing comparatively lessens according to the progress of polishing. (3) Nitrogen free extracts increase in accordance with the advance of polishing process.

22. M. Sawamura, R. Kurosawa :- J. of Scient. Agricult. Society. No. 195. The relation between the saccharification and the viscosity of the starch paste and the shape of the starch granule. The starch, which shows a strong viscosity in the state of paste, is slow as to its saccharification, but there is no regular rule among them, except cereals.

23. I. Kawasaki :- J. of Scient. Agricult. Society. No. 198.

The experiment on the movement of water is rice grains (Preliminary report). The volume weight of a husked rice, when moistened and afterward dehydrated by chemicals, is inversely proportional to the content of water. Brine does not give any bad effect to the quality of rice.

24. S. Kato, J. Ishikawa :- J. of Scient. Agricult. Society. No. 199.

The relation between the degree of dryness of unhulled or raw rice and husked rice, and a volume weight of them. Both unhulled and husked rice increase their volume weights in parallel with the degree of dryness.

25. M. Sawamura, R. Kurosawa ;- J. of Scient. Agricult. Society. No. 200. Digestibility of polished rice, husked rice, sweet potatoe, tangle (lami- 
noria), and Cortinellus Shiitake. The husked rice is less digestible compared with the polished rice.

26. Y. Noguchi :- J. of Scient. Agricult. Society. No. 245.

On the enzymes of preserved rice. The undermentioned facts are recognised in the rice during one and half years after the harvest.

(1) The activity of diastase somewhat suddenly decrease in July of the year after the harvest. After that time no considerable change is perceived, since the activity of diastase remained during one and half years after the harvest. (2) The activity of lipase and peroxidase, too, decreases gradually in accordance with the period of preservation and somewhat suddenly lessens in August of the year after the harvest. (3) The activity of catalase, too, lessens gradually, but no sudden change occurs. (4) The activity of oxydase does not change. (5) Either on unhulled or on husked rice the activities of these five enzymes do not seem to be affected by the change in the degree $o$ dryness and the mode of preservation.

27. S. Sugihara :- J. of Scient. Agricult. Society. No. 248, No. 249, No. 250. Physical investigations of cereals and their principal constituedts.

(1) A volume weight of husked rice when dried by some drying agent increases inversely to its water contents until the water contents are diminished to about $5-6 \%$. But under $5 \%$ of water contents, the volume weight decreases proportionally to the water contents of rice. The reason of this phenomenon, is due to the fact that husked rice has the greatest density when it contains $5-6 \%$ of water while it has less density when it contains water less than $5 \%$ because the surface of rice grain becomes considerably rough when so dried and grains can not adhere closely one another. (2) A volume weight of polished or husked rice, is inversely proportional to its water contents. (3) A volume weight of polished rice decreases inversely to the quantity of sand used in the polishing process. (4) Dilute ethyl alcohol is the best liquid to prevent air bubbles adhered on the surface of husked rice. (5) Among many kinds of starches, potatoe starch has the greatest hygroscopicity, next comes the starch of an arrow root and a sweet potatoe, while the starch of rice, wheat and maize has the least hygroscopicity.

28. K. Goto:- J. of Scient. Agricult. Society. No. 265, On enzymes and chemical compositions of husked rice in several periods of ripening.

(1) A yellow riped rice seemes to contain somewhat greater quantity of sugar compared with that of a full ripened and the quantity of 
starch is greater, while ash, protein, and crude fibre are less in the former. The activity of the enzymes lies between the milky and the dead ripen ones. (2) Rice of a dead ripe has the greatest diastatic power and the least peroxidase power. (3) Rice of milky ripe has a greater quantity of ash, fat, protein and fibre, while the least quantity of starch. Activity of diastase is the weakest, while that of peroxidase is the strongest by this period.

29. K. Aso :- R. of Imp. Agricult. Exp. Station. No. 451 st.

Experiments on the enzymes in husked rice. Enzymes exist in husked rice are as follows.

(1) Oxydase, peroxidase, catalase, diastase, dextrinase, maltase, invertin, raffinase, lipase, urease, phytase and a protein splitting enzyme in a neutral medium. (2) Peroxydase is vigorous in a newly cropped rice and gradually lessens in accordance with the lapse of time. (3) Chlorine and formalin gases injure the function of lipase, diastase, and peroxidase, while cyanogen gas injure the action of peroxidase only. Chlorpicrin has no any influence for all these enzymes described above.

30. M. Aoi :- R. of Imp. Agricul. Exp. Station. No. 451 st.

Investigations on the origin of disease in red changed rice.

(1) A fungus causes this disease belongs to the genus of oospora. (2) Pigment produced by the fungus exists in cell membrance and it does not dissolve in water, alcohol and ether. When made alkaline, the colour becomes reddish purple, but it recovers its original colour when acidified. (3) The optimum temperature for the growth of the fungus lies $24-28^{\circ} \mathrm{C}$, the maximum being $35^{\circ} \mathrm{C}$ and the mimimum $11^{\circ} \mathrm{C}$. (4) No poisonous effect was observed when two adult men ate $200 \mathrm{~g}$. each, every day during a week.

31. H. Kitamura :- R. of Imp. Agricul. Exp. Station. No. 43.

Investigations on the fatty matters in rice bran.

(1) Fatty matters in rice bran principally exist as glycerin esters of unsaturated fatty acids as oleic and linoleic acid and of saturated fatty acid as palmitic, moreover there exist a small quantity of phytosterol. It may, therefore, be supposed that the easily decomposable matter in rice bran should be olein and linolein. (2) The constants about fatty matters of rice are as follows.

$\begin{array}{lrlr}\text { Acid number } & 31.21 & \text { Iod numler } & 104.88 \\ \text { Saponification number } & 184.76 & \text { Hehner's number } & 95.65 \\ \text { Ester number } & 153.55 & \text { Reichert meissl number } & 0.55\end{array}$

32. K. Aso, T. Nakoshi :- J. of Scient. Agricul. Society. No. 272.

The investigation of the enzymes of husked rice (continued). Also the 
judgment of rice whether new or old.

(1) On the peroxide enzyme of husked rice newly cropped or old. $2 \mathrm{~g}$. of husked rice, grinded, mixed with 20c.c. water and tested about 5c.c. filtrate. (a) Reaction of guaijacum tincture-few drops of $1 \%$ solution of the tincture, a few drops of hydrogen peroxide solution of $1.5 \%-$ indigo colouration. (b) Reaction of guaijacol-few drops of $1 \%$ solution of guaiacol, a few drops of hydrogen peroxide solution of $1.5 \%$ reddish brown colouration. (c) Reaction of pyrogallol-few drops of $1 \%$ pyrogallic water solution, a few drops of $1.5 \%$ hydrogen perxide solution-yellow colouration. (d) Reaction of paraphenylene diaminea few drops aqueous solution of $1 \%$ paraphenylene diamine hydrochloride, a few drops of aqueous solution of sodium acetate, a few drops of $1.5 \%$ hydrogen peroxide solution-purple colouration. In the above reactions, newly cropped rice takes colour very well, but rice elapsed few years after crop, is coloured faintly, while rice preserved for a long years shows no colouration.

(2) On the catalase of husked rice newly cropped or old. Newly cropped husked rice indicates a strong reaction for catalase, but the husked rice elapsed one year after the crop shows the reaction in a considerably less degree. (3) On the reducing enzymes of husked rice newly harvested or old. Newly harvested husked rice indicates the reaction of a reducing enzyme distinctly, while that elapsed one year after the crop, shows no reaction. (4) On an ureasplitting enzyme of husked rice newly cropiped or old. Newly harvested husked rice indicates the presence of an ureasplitting enzyme distinctly, but that elapsed three years after the crop indicates no reaction. (5) Relation of heat to enzymes in husked rice. There occurs no change of diastatic power of raw or husked rice, below $70^{\circ} \mathrm{C}$ in 3 hours. (a) Diastatic power in husked rice undergoes no perceptible change, when heated during three hours under $70^{\circ} \mathrm{C}$, while it is remarkably retarded by heating three hours above $100^{\circ} \mathrm{C}$. (b) Urease in husked rice is somewhat retarded in its action by heating above $80^{\circ} \mathrm{C}$ during two hours, while considerably retarded by heating above $100^{\circ} \mathrm{C}$ during three hours. (c) Phytase in husked rice is retarded in its action by heating at $100^{\circ} \mathrm{C}$ during three hours, when heated above $100^{\circ} \mathrm{C}$ during three hours, the reaction is evidently lessened. (d) Peroxidase in husked rice is retarded in its action by heating above $80^{\circ} \mathrm{C}$ during three hours, above $100^{\circ} \mathrm{C}$ during three hours, the reaction is remarkably decreased.

(6) Relation between the enzymes and the water contents of husked rice during storage. Activity of peroxide enzyme undergoes no any 
influence, if water contents of husked rice are below $13 \%$, while above $14 \%$ gradually decreasses its activity, if above $18 \%$ is distinctly retarded. Catalase, lipase, urease, phytase and diastase, too, indicate the same behaviour. (7) Mode of judgment of rice husked or polished, whether newly harvested or old. A large quantity of aqueous solution of $1 \%$ guaiacol, mixed with 20 grains of rice either husken or polished, stirred and is added with a drop of hydrogen peroxide solution-a newly cropped rice both husked and polished indicates reddish brown colouration on the parts of embryo and bran, but rice cropped in the previous year takes colour slightly on the part of embryo only.

33. Y. Kozai, H. Ando, U. Suzuki, T. Shimamura:- R. of Imp. Agricul. Exp. Station (extra) July 1910.

An investigation on a disease like beriberi of birds. Also, value of polished rice as foodstuff. The investigation has been begun in 1904 . Phosphorus in rice bran exist in the form of phytin. The majority of iron exists, combined with a digestible protein, which is named as "ferroglobulin" Iime and magnesia are comprised chiefly in phytin, while the greater part of potassium exists as an inorganic compound. When birds arc fed with the ferroglobulin extracted from rice bran, with moderate quantities of phytin, lecithin, potassium salt, sodium chloride and calcium carbonate, a disease like beriberi can be avoided or cured. As polished rice is unfit as a sole foodstuff, it is important to compensate the defect by selection of proper side dishes or by other methods.

34. K. Kondo :- J. of Societ, of Agricul. and Forest. Sapporo. No. 59.

On the protein in polished rice. Experiments were made on the refractivity of an alkaline solutions of both ordinary and glutinous rice. The refractivity changes in proportional to the density of a protein provided temperature and density of alkality are constant. Furthermore elementary analysis of protein were made.

35. T. Tadokoro, Y. Nakamura :- J. of Societ. of Agricul. and Forest. Sapporo. No. 65 , No, 68 , No. 72.

On oryzenin in ordinary and glutinous rice. Oryzenin of polished, ordinary rice is poor in carboxyl group, while rich in amino-acids group, compared with that of glutinous. Change caused by ultraviolet ray, is greater in the glutinous compared with the ordinary rice. When iodoprotein is prepared the protein of glutinous rice combines with much iodine than the ordinary one. Oryzenin of ordinary rice contain much ash than that of glutinous one. Iso electric points differ according to oryzenins of the different sources. That of ordinary 
one approaches to a cathode, while that of glutinous one approaches to an anode. About the quantity of amino-acids number, oryzenin of ordinary rice is rich in ammonia form, while that of glutinous one is rich in mono-amino acid form when they are compared each other. Oryzenin of ordinary rice is always rich in nitrogen contents than that of glutinous one, when examined as the products of acetylifacation.

36. T. Tadokoro, Y. Nakamura, S. Watanabe:- J. of Collage. of Agricul. Hokkaido, Imp. Univers. Vol. XIV No. 3. Difference of the physical and chemical properties between glutinous and ordinary rice oryzenin. (1) As the refractvity of an alkaline solution of ordinary rice is greater than that of glutinous one, the former is acknowledged to be denser in its optical construction compared with the latter. (2) As the quantity combined of hydrochloric acid with oryzenin, after Cohnheim's method, is greater in ordinary rice than that of glutinous one, the former has the greater quantity of amino acids group to combine with hydrochloric acid, than the latter. (3) Ash contents of oryzenin of ordinary rice is greater than that of glutinous one. Ordinary rice has much sulphur and less phosphorus compared with glutinous one. (4) When amino acids are estimated, ordinary rice oryzenin contains. much nitrogen belonging to ammonia, arginin and lysine, while glutinous one contains much nitrogen belonging to monoamino acids, histidin and cystin.

37. T. Tadokoro, T. Takahashi, Y. Nakamura, S. Watanabe :- J. of Societ. Agricul. and Forest. Sapporo, No. 68.

Difference of the physical and chemical properties of starch between glutinous and ordinary rice. (1) Inorganic constituents of ordinary rice starch are greater than glutinous one. (2) Absorptive power of iodine in iod-alcohol by starch grain, is always greater in ordinary rice than in glutinous one. (3) Absorptive power of iodine in iodiod-potassium solution, too, is greater in odinary rice than in the latter. (4) Heat of combustion is higher in ordinary rice starch than in the latter.

38. T. Tadokoro, S. Sato :- J. of Collage of Agricul. Hokkaido, Imp. Univers. Vol. XIII. No. 1.

On the difference of colloidality between glutinous and ordinary rice starch. (1) The swelling power of starch suspensoid in iodine solution is smaller in ordinary rice than in glutinous one. The former has strong resistance for sulphuric acid. (2) The affinity of glutinous starch to iodine is smaller compared to that of ordinary one. (3) The 
absorptive power of glutinous starch to iodine is smaller than that of ordinary one.

39. U. Suzuki, T. Shimamura, S. Ohdake :- J. of Tokio. Chem. Societ. Band 32, 34.

On oryzanin, a constituent of rice bran. The four substances such as carbohydrate, protein, fat and inorganic salts have been regarded generally for a long time, as four indispensable nutritious factors. Nevertheless, by the results of many animal experiments carried out by them, it has been proved that animals can not achieve the complete development by those factors only. They experienced that animals can thrive completely, if a small quantity of an alcoholic extract of rice bran is added to those four factors mentioned above. Dr. Suzuki named this indispensable principle as oryzanin. This experiment has brought an epoch making discovery in the theory of nutrition. Oryzanin has, according to an elementary analyses, the molecular formula $\mathrm{C}_{18} \mathrm{H}_{10} \mathrm{~N}_{2} \mathrm{O}_{9}$, and indicates strong diazo and Millon's reactions, a purple red colouration by ferric chloride, a slight green colouration by phosphomolybdic acid, a deep indigo colouration by ammonia and decolourises a blue colouration of iod-starch.

40. K. Sugimoto :- R. of Instit. of Nutrition. Vol. I No. 2.

Relation between a degree of polishing of rice and a digestive absorption coefficient. (1) A digestive absorption coefficient of every constituent in rice is increased provided the polishing process being advanced. (2) When equal quantities of rice having several different degree of polishing, are boiled and eaten, an actual quantity of the constituents (except carbohydrates) absorbed, decreases as the polishing degree proceeds; while of the nitrogenous matter, it is nearly the same in the rice of $7 \%$ loss of polishing, the half polished and the husked. (3) The quantity of materials excreted is remarkably decreased as the polishing degree proceeds. In husked rice it is particularly abundant. Rate of excreted matter of the constituents to the actual absorbed quantity, shows the highest percentage in the hushed rice, and then gradually decreases as the polishing degree proceeds. .

41. K. Sugimoto, M. Higuchi, S. Momoeda, Y. Hota, S. Tanaka :- R. of Institut. of Nurtition. Vol. I, No. 2.

Relation between an art of cookery of rice and a digestive absorption coefficient. (1) A digestive absorption coefficient is increased when rice is eaten as a dumpling (rice is powdered mixed with water, rounded to a ball, boiled) than as boiled rice, no matter husked or boiled, Boiled polished rice is better than a husked rice dumpling 
in an absorption coefficient of each constituent. (2) When polished rice is eaten as a gruel, the digestive absorption coefficient is decreased in total nitrogen, carbohydrates and fat than eaten as boiled rice, but the absorption coefficient of ash is better in the former. (3) Digestive absorption coefficient is decreased in total nitrogen and carbohydrates, and increased in fat and ash, when polished rice is caten as a porridge than as boiled rice. (4) When polished rice is eaten as Sushi (boiled rice mixed with. fish, vegetables and vinegar), the digestive absorption coefficient is somewhat increased than as boiled rice. (5) When polisher rice is eaten as Sekihan (rice and red bean are mixed and steamed) the digestive absorption ccefficient is inferior compared with ordinary boiled rice. (6) Of steamed rice (polished glutinous rice 7 pt. polished ordinary rice 3 pt.) and boiled polished rice, the former is somewhat inferior in the absorption coefficient of the total nitrogen, fat and ash, while that of corbohydrates are almost equal. (7) Glutinous rice when eaten as Mochi (rice steamed, kneaded to a mass or to a cake) the digestive absorption coefficient of each constituent is increased than the steamed rice.

42. F. Ando, H. Terada, S. Masuda, Y. Hoshino, C. Kanomata, H. Satow :R. of Brew, Exp. Instit. No. 3, 4, 9, 10, 11, 12.

They investigated physical and chemical properties on a few kinds of polished rice used for Saké brewing.

43. T. Takahashi, H. Satow:- R. of Brew. Exp. Instit. No. 38.

On the chemical composition of polished rice, with special reference to the nutritive value of its protein matters for Saké yeast and Aspergillus Oryzae. Besides general chemical constituents of polished rice used for Saké brewing, four kinds of protein such as albumin, globulin, prolamin, and oryzenin were isolated. These four proteins were submitted to elemental analyses. Three proteins other than prolamin were utilized by the fungi.

44. T. Takahashi, H. Satow ;- R. of Brew. Exp. Instit. No. 64.

About tyrosin content in polished rice in regard to the quality of rice as raw material of Saké brewing. Since tyrosin in polished rice may be taken as an origin of bitter taste (tyrosol) of Saké, they tried to determine the quality of rice used for Sake brewing by the contents of tyrosin. This report is a preliminary one.

45. T. Tadokoro, S. Watanabe :- J. of Societ. of Agricul. and Forest, Sapporo. No. 65.

On a polishing degree of rice used for saké brewing and its protein contents. As protein matter among many important constituents of 
rice, passes gradually into bran constituents in accordance with the degree of polishing, so it may be indicated the degree of polishing by the quantity of protein.

46. T. Tadokoro, Y. Nakamura, S. Watanabe :- J. of Societ. of Agricul. and Forest. Sapporo, No. 70, No. 72.

On the special characters of protein and starch in the varieties of rice used for Sake brewing. (1) The peculiarities of oryzenin in the varieties of rice used for Saké brewing, are as follows.-Always scanty in quantity of ash, differ in amino acid numbers, viz. poor in ammonia, melanin and lysine forms, while rich in histidin and cystin forms, compared to ordinary rice used as a food stuff. (2) The viscosity of starch paste of rice used for Sake brewing, is higher than that of oreinary rice used as food stuff. The quantity of ash of the former is poorer than that of the latter. (3) The quatity of silver in the silver salt of oryzenin of rice used for Sake brewing, is always greater when compared with the other. (4) The quantity of ash of oryzenin of rice used for Saké brewing is always less than the other. (5) Oryzenin of rice used for Saké brewing is poor in nitrogen in monoamino, arginin and lysin forms, while rich in histidin and cystin forms, compared with that of the ordinary variety. 